Supporting Information

\title{
Nucleophilic Activation of Hydrosilanes via a Strain-imposing Strategy Leading to Functional Sila-aromatics
}

\author{
${\text { Toshiya Ikeuchi, }{ }^{\dagger} \text { Keiichi Hirano }}^{*,+}$ and Masanobu Uchiyama ${ }^{*,+, \neq, \S}$ \\ ${ }^{+}$Graduate School of Pharmaceutical Sciences, The University of Tokyo, 7-3-1 Hongo, Bunkyo-ku, Tokyo 113-0033, Japan \\ ₹Cluster for Pioneering Research (CPR), Advanced Elements Chemistry Laboratory, RIKEN, 2-1 Hirosawa, Wako-shi, Saitama 351-0198, Japan

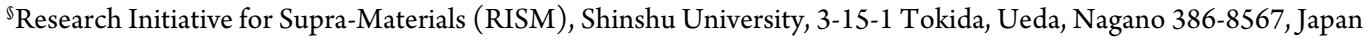

\section{Table of Contents}

1 General

2 Experimental Details

2-1. Preparation of Cyclic Hydrosilanes

2-2. Preparation of 1-Br-2-alkynylbenzenes

2-3. Hydrosilylation Leading to Diverse Benzosiloles (Table 2)

2-4. Transformation of Benzosilole (Scheme 3)

2-5. Hydrosilylation for Other Silacycles (Table 3)

2-6. Absorption and Emission Spectra of 3a and 11

2-7. Computational Studies (Figure 1, Scheme 2, Table 3, and Scheme S1)

2-8. Mechanistic Studies 


\section{General}

\section{- Instrumentation.}

NMR spectra were obtained on a Bruker AVANCE III HD 500 spectrometer and a Bruker Ascend 400 spectrometer. Chemical shifts are expressed in $\delta(\mathrm{ppm})$ values, and coupling constants are expressed in hertz $(\mathrm{Hz}) .{ }^{1} \mathrm{H},{ }^{13} \mathrm{C}$, and ${ }^{29} \mathrm{Si}$ NMR spectra were referenced to tetramethylsilane as an internal standard. The following abbreviations are used: $\mathrm{s}=$ singlet, $\mathrm{d}=$ doublet, $\mathrm{t}=$ triplet, quint $=$ quintet, $\mathrm{sep}=$ septet, $\mathrm{m}=$ multiplet, $\mathrm{br} \mathrm{s}=$ broad singlet, and br $\mathrm{d}=\mathrm{broad}$ doublet. Melting points were determined with OptiMelt Automated Melting Point System and uncorrected. IR spectra were obtained on a JASCO FT/IR-4700 spectrophotometer. ESI mass spectra were measured on a Bruker micrOTOF-II spectrometer. GCMS: Mass spectra were obtained on either an Agilent 7890A/5975C or 7890B/5977A spectrometers. Electronic absorption spectra were measured using a JASCO V-670. Emission spectra were recorded on a JASCO FP-8500 spectrofluorometer. The absolute fluorescence quantum yield $\left(\Phi_{\mathrm{FL}}\right)$ was measured using an integrating sphere. Yamazen medium pressure liquid chromatography (MPLC) system (EPCLC-W-Prep 2XY A-Type) and JAI Recycling Preparative HPLC (GPC) (LC-9110 II NEXT, mobile phase: chloroform) were used for purification of products. Data collections for X-ray analysis were performed at $90 \mathrm{~K}$ on a RIGAKU XtaLAB Synergy-S diffractometer.

\section{- Materials.}

Unless otherwise noted, materials were purchased from FUJIFILM Wako Pure Chemical Corporation, Tokyo Chemical Industry Co., Ltd., Sigma-Aldrich Co., LLC., and other commercial suppliers. ${ }^{t}$ BuLi in ${ }^{n}$ pentane was obtained from Kanto Chemical Co., Inc. 2,5-Dimethyl-2,5-hexanediol (for the synthesis of 1a) and 2,4-dimethyl-2,4-pentanediol (for the synthesis of $\mathbf{1 b}$ ) were purchased from Tokyo Chemical Industry Co., Ltd.

Anhydrous THF was purchased from Kanto Chemical Co., Inc. All chemicals were of reagent grade and used as received. Air- and moisture-sensitive manipulations were performed with standard Schlenk techniques under argon atmosphere. Medium pressure liquid chromatography was performed using the disposable Hi-flash premium column from Yamazen Co., and preparative thin-layer chromatography was carried out using Merck Silica Gel $60 \mathrm{PF}_{254}$, and thin-layer chromatography was carried out on $0.25 \mathrm{~mm}$ Merck silica gel plates (60F-254). 


\section{Experimental Details}

\section{2-1. Preparation of Cyclic Hydrosilanes}

4,4,7,7-tetramethyl-2-phenyl-1,3,2-dioxasilepane (1a)

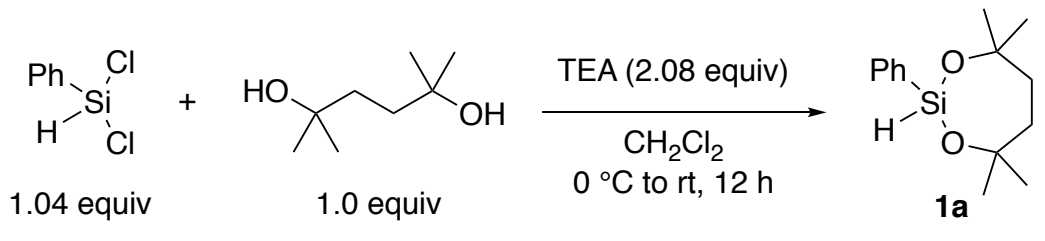

To a heat gun-dried 2-necked $200 \mathrm{~mL}$ egg-plant-shaped flask were added dichloro(phenyl)silane (5.31 g, $30.0 \mathrm{mmol}$, 1.04 equiv) and $\mathrm{CH}_{2} \mathrm{Cl}_{2}(20 \mathrm{~mL})$. After the solution was cooled to $0{ }^{\circ} \mathrm{C}$, triethylamine $(8.3 \mathrm{~mL}, 60.0 \mathrm{mmol}, 2.08$ equiv) were added. To the mixture was added a solution of 2,5-dimethylhexane-2,5-diol (4.21 g, $28.8 \mathrm{mmol}, 1.0 \mathrm{equiv}$ ) in $\mathrm{CH}_{2} \mathrm{Cl}_{2}(80 \mathrm{~mL})$ dropwise at $0{ }^{\circ} \mathrm{C}$. The resulting mixture was allowed to warm to room temperature and vigorously stirred for $12 \mathrm{~h}$. After volatiles were removed by evaporation, the residue was dissolved in hexane or pentane and stirred vigorously for 12 hours. Celite filtration and evaporation gave slightly yellow oil. The residue was purified by Kugelrohr distillation at $120^{\circ} \mathrm{C}$ under a pressure of $4.0 \times 10^{-1} \mathrm{mmHg}$ to give $1 \mathrm{a}(6.60 \mathrm{~g}, 26.4 \mathrm{mmol})$ in $88 \%$ yield as colorless oil. ${ }^{1} \mathbf{H}$ NMR (500 MHz, $\left.\mathbf{C D C l}_{3}\right): \delta 1.28(\mathrm{~s}, 6 \mathrm{H}), 1.38(\mathrm{~s}, 6 \mathrm{H}), 1.86(\mathrm{~s}, 4 \mathrm{H}), 5.04(\mathrm{~s}, 1 \mathrm{H}), 7.33-7.40(\mathrm{~m}, 3 \mathrm{H})$, 7.64-7.66 (m, 2H). ${ }^{13} \mathbf{C}$ NMR (125 $\mathbf{M H z}, \mathbf{C D C l}_{3}$ ): $\delta 29.8$ (br), 30.9 (br), 38.0, 75.5, 127.9, 130.2, 134.0, 135.3. IR (ATR-IR): 2974, 2933, 2151, 1430, 1385, 1367, 1277, 1243, 1212, 1186, 1122, 1025, 857, 836, 738, 698, 542 $\mathrm{cm}^{-1}$. HRMS (ESI): $m / z$ calculated for $\mathrm{C}_{15} \mathrm{H}_{26} \mathrm{NaO}_{3} \mathrm{Si}^{+}[\mathrm{M}+\mathrm{MeOH},+\mathrm{Na}]^{+}=305.1543$, found: 305.1547 .

\section{4,4,6,6-tetramethyl-2-phenyl-1,3,2-dioxasilinane (1b)}<smiles>CC1(C)CC(C)(C)O[SiH](c2ccccc2)O1</smiles>

Prepared following the protocol for 1a. $89 \%$ yield ( $5 \mathrm{mmol} \mathrm{scale}$, colorless oil, Kugelrohr: $115^{\circ} \mathrm{C}, 4.0 \mathrm{x}$ $\left.10^{-1} \mathrm{mmHg}\right) ;{ }^{1} \mathbf{H}$ NMR (400 MHz, $\left.\mathbf{C D C l}_{3}\right): \delta 1.41(\mathrm{~s}, 6 \mathrm{H}), 1.46(\mathrm{~s}, 6 \mathrm{H}), 1.96(\mathrm{~s}, 2 \mathrm{H}), 5.20(\mathrm{~s}, 1 \mathrm{H})$, 7.27-7.46 (m, 3H), 7.65-7.67 (m, 2H). ${ }^{13} \mathbf{C}$ NMR (100 $\mathbf{~ M H z , ~} \mathbf{C D C l}_{3}$ ): $\delta$ 32.0, 33.0, 52.3, 74.1, 128.1, 130.9, 133.8, 134.9. IR (ATR-IR): 2973, 2139, 1592, 1430, 1384, 1367, 1347, 1300, 1241, 1193, 1122, 1020, 958, 862, 823, 736, 697, $583 \mathrm{~cm}^{-1}$. HRMS (ESI): $m / z$ calculated for $\mathrm{C}_{14} \mathrm{H}_{24} \mathrm{NaO}_{3} \mathrm{Si}^{+}[\mathrm{M}+\mathrm{MeOH},+\mathrm{Na}]^{+}=$ 291,1387, found: 291.1389 . 


\section{2-2. Preparation of 1-Br-2-alkynylbenzenes}

1-Bromo-2-(phenylethynyl)benzene 2a, ${ }^{1}$ 1-bromo-2-((4-chlorophenyl)ethynyl)benzene, ${ }^{1}$

1-bromo-2-((4-fluorophenyl)ethynyl)benzene, ${ }^{2}$ 1-bromo-2-((4-(trifluoromethyl)phenyl)ethynyl)benzene, ${ }^{3}$

1-bromo-2-((4-butylphenyl)ethynyl)benzene, ${ }^{4}$ 1-bromo-2-((4-methoxyphenyl)ethynyl)benzene, ${ }^{1}$

2-((2-bromophenyl)ethynyl)-1,3,5-trimethylbenzene, ${ }^{5}$ 1-((2-bromophenyl)ethynyl)naphthalene, ${ }^{2}$

2-((2-bromophenyl)ethynyl)thiophene, ${ }^{6}$ 1-bromo-2-(phenylbuta-1,3-diyn-1-yl)benzene, ${ }^{7}$

1,4-bis((2-bromophenyl)ethynyl)benzene, ${ }^{8}$ 2-((2-bromophenyl)ethynyl)-1,1'-biphenyl, ${ }^{6}$ and

1-bromo-8-(phenylethynyl)naphthalene ${ }^{9}$ were prepared according to the reported protocol.

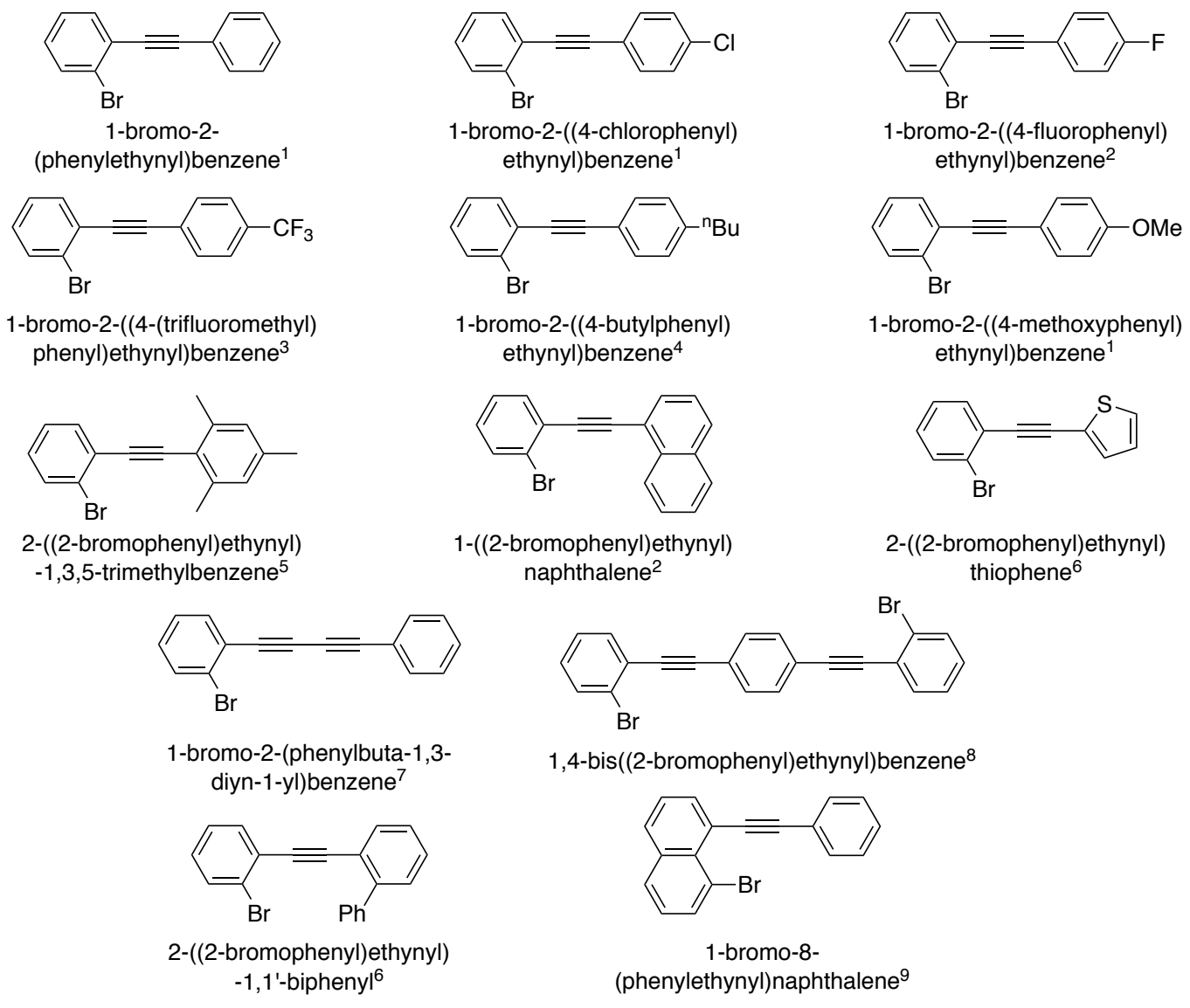

(1) Chintawar, C. C.; Mane, M. V.; Tathe, A. G.; Biswas, S.; Patil, N. T. Org. Lett. 2019, 21, 7109.

(2) Shaikh, A. C.; Ranade, D. S.; Rajamohanan, P. R.; Kulkarni, P. P.; Patil, N. T. Angew. Chem. Int. Ed. 2017, 56, 757.

(3) Chen, M.; Su, N.; Deng, T.; Wink, D. J.; Zhao, Y.; Driver, T. G. Org. Lett. 2019, 21, 1555.

(4) Verma, A. K.; Jha, R. R.; Chaudhary, R.; Tiwari, R. K.; Reddy, K. S. K.; Danodia, A. J. Org. Chem. 2012, 77, 8191.

(5) Ung, G.; Soleilhavoup, M.; Bertrand, G. Angew. Chem. Int. Ed. 2013, 52, 758.

(6) Naveen, K.; Perumal, P. T.; Cho, D. Org. Lett. 2019, 21, 4350.

(7) Liang, Y.; Meng, T.; Zhang, H.; Xi, Z. Synlett 2011, 7, 911.

(8) Iwaniuk, D. P.; Wolf, C. J. Am. Chem. Soc. $2011,133,2414$.

(9) Yang, Q.; Liu, L.; Chi, Y.; Hao, W.; Zhang, W.-X.; Xi, Z. Org. Chem. Front. 2018, 5, 860. 


\section{- General Procedure:}

To a $100 \mathrm{~mL}$ greaseless Schlenk tube were added 1-bromo-2-iodebenzene ( $849 \mathrm{mg}, 3.0 \mathrm{mmol}, 1.0 \mathrm{equiv}$ ), CuI (19.0 mg, $0.033 \mathrm{mmol}, 3.3 \mathrm{~mol} \%), \mathrm{PdCl}_{2}\left(\mathrm{PPh}_{3}\right)_{2}(42.1 \mathrm{mg}, 0.020 \mathrm{mmol}, 2.0 \mathrm{~mol} \%)$, and the tube was evacuated and backfilled with argon three cycles. Triethylamine $(20 \mathrm{~mL})$ and a terminal alkyne $(1.0 \mathrm{mmol}$, 1.0 equiv $)$ were successively added. The resultant mixture was stirred at room temperature overnight. The reaction was concentrated in vacuo and the crude residue was dissolved in $\mathrm{CH}_{2} \mathrm{Cl}_{2}$, pre-adsorped on $\mathrm{SiO}_{2}$, chromatographed with MPLC (eluted typically with a mixture of hexane and EtOAc). If required, materials were further purified with the recycling preparative HPLC (GPC).

\section{1-((2-bromophenyl)ethynyl)-3,5-bis(trifluoromethyl)benzene}

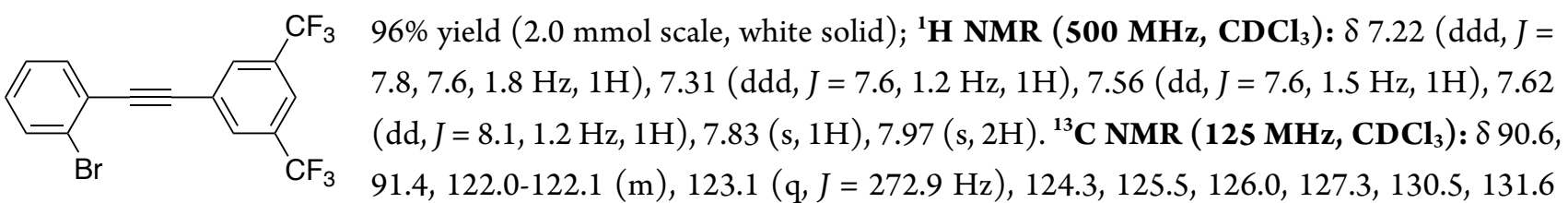
(apparent d, $J=2.7 \mathrm{~Hz}), 132.2(\mathrm{q}, J=33.6 \mathrm{~Hz}), 132.8,133.7 .{ }^{19} \mathbf{F}$ NMR (470 $\left.\mathbf{M H z}, \mathbf{C D C l}_{3}\right): \delta-63.2$. IR (ATR-IR): 1473, 1426, 1384, 1280, 1165, 1123, 1043, 1025, 925, 898, 847, 760, 715, 698, 683, 657, $553 \mathrm{~cm}^{-1}$. HRMS $^{2}$ (ESI): $m / z$ calculated for $\mathrm{C}_{16} \mathrm{H}_{7} \mathrm{AgBrF}_{6}^{+}[\mathrm{M}+\mathrm{Ag}]^{+}=498.8681$, found: 498.8674 .

\section{(E)-1-bromo-2-(4-phenylbut-3-en-1-yn-1-yl)benzene}

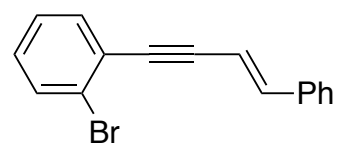

$61 \%$ yield (1.92 mmol scale, off-white solid); ${ }^{1} \mathbf{H}$ NMR (400 $\mathbf{M H z}, \mathbf{C D C l}_{3}$ ): $\delta 6.43$ (d, J= $16.1 \mathrm{~Hz}, 1 \mathrm{H}), 7.11(\mathrm{~d}, J=16.1 \mathrm{~Hz}, 1 \mathrm{H}), 7.16(\mathrm{ddd}, J=8.0,7.7,1.7 \mathrm{~Hz}, 1 \mathrm{H}), 7.27-7.37(\mathrm{~m}$, $4 \mathrm{H}), 7.43-7.45(\mathrm{~m}, 2 \mathrm{H}), 7.50(\mathrm{dd}, J=7.7,1.7 \mathrm{~Hz}, 1 \mathrm{H}), 7.60(\mathrm{dd}, J=8.0,1.2 \mathrm{~Hz}, 1 \mathrm{H}) .{ }^{13} \mathbf{C}$

NMR (100 MHz, $\left.\mathbf{C D C l}_{3}\right): \delta$ 90.3, 93.6, 107.9, 125.6, 125.7, 12.6, 127.2, 128.9, 129.0, 129.4, 132.6, 133.4, 136.3, 142.3. IR (ATR-IR): 3060, 3027, 1469, 1447, 1433, 1026, 952, 774, 747, 689, 542, 520, 511, 498, 482, 465, 454, 438, 420, 412, $402 \mathrm{~cm}^{-1}$. HRMS (ESI): $m / z$ calculated for $\mathrm{C}_{16} \mathrm{H}_{11} \mathrm{AgBr}^{+}[\mathrm{M}+\mathrm{Ag}]^{+}=388.9090$, found: 388.9095 .

\section{((2-bromophenyl)ethynyl)(4-(tert-butyl)phenyl)sulfane}

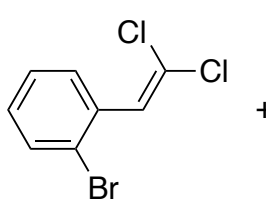

1.07 equiv

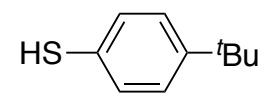

1.0 equiv

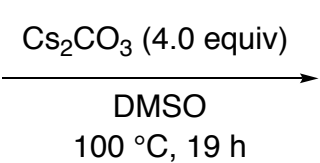

$100{ }^{\circ} \mathrm{C}, 19 \mathrm{~h}$

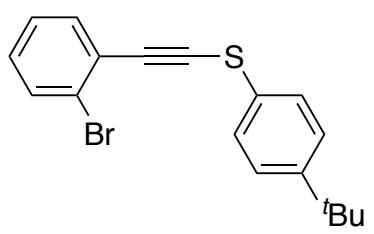

According to the protocol by Pan et al., ${ }^{10}$ were added 1-bromo-2-(2,2-dibromovinyl)benzene (592.9 mg, 1.07 equiv, $1.74 \mathrm{mmol})^{11}$ and $\mathrm{Cs}_{2} \mathrm{CO}_{3}(2.12 \mathrm{~g}$, 4.0 equiv, $6.52 \mathrm{mmol}$ ) into DMSO (10 mL). 4-(Tert-butyl)benzenethiol (271 mg, 1.0 equiv, $1.63 \mathrm{mmol}$ ) was added to the mixture, and the reaction vessel was purged with argon. The resultant reaction mixture was stirred at $100{ }^{\circ} \mathrm{C}$ for $19 \mathrm{~h}$, cooled to rt, quenched with water under vigorous stirring. Aqueous layer was extracted with hexane $\mathrm{x} 3$, and the combined organic phase was washed with water $\mathrm{x} 1$, dried over $\mathrm{Na}_{2} \mathrm{SO}_{4}$, filtered, and concentrated to give yellow oil. Purification with MPLC (eluent: hexane) gave the desired product as colorless oil in $60 \%$ yield (337 mg). ${ }^{1} \mathbf{H}$ NMR (400 $\mathbf{~ M H z , ~} \mathbf{C D C l}_{3}$ ): $\delta 1.32(\mathrm{~s}, 9 \mathrm{H}), 7.16(\mathrm{dt}, J=7.8,1.6 \mathrm{~Hz}, 1 \mathrm{H}), 7.27$ (dt, J= 7.5, 1.1

(10) Ni, Z.; Wang, S.; Mao, H.; Pan, Y. Tetrahedron Lett., 2012, 52, 3907.

(11) Hojo, D.; Tanaka, K. Org. Lett., $2012,14,1492$. 
$\mathrm{Hz}, 1 \mathrm{H}), 7.39(\mathrm{~d}, J=8.5 \mathrm{~Hz}, 2 \mathrm{H}), 7.48-7.50(\mathrm{~m}, 1 \mathrm{H}), 7.50(\mathrm{~d}, J=8.5 \mathrm{~Hz}, 2 \mathrm{H}), 7.60(\mathrm{dd}, J=8.0,1.0 \mathrm{~Hz}, 1 \mathrm{H}) .{ }^{13} \mathbf{C} \mathbf{~ N M R}$ (100 MHz, $\mathbf{C D C l}_{3}$ ): $\delta 31.3,34.5,81.4,95.9,125.0,125.3,126.4$ (overlapped), 127.0, 128.8, 129.3, 132.4, 133.0, 150.1. IR (ATR-IR): 2962, 2902, 2867, 2170, 1584, 1496, 1466, 1433, 1399, 1363, 1267, 1199, 1116, 1047, 1025, 1011, 822, $751,683,643,589,544,525,510,497,470,458,441 \mathrm{~cm}^{-1}$. HRMS (APCI): $m / z$ calculated for $\mathrm{C}_{18} \mathrm{H}_{18} \mathrm{BrS}^{+}[\mathrm{M}+\mathrm{H}]^{+}=$ 345.0307, found: 345.0306 .

\section{((2-bromophenyl)ethynyl)diphenylphosphane}

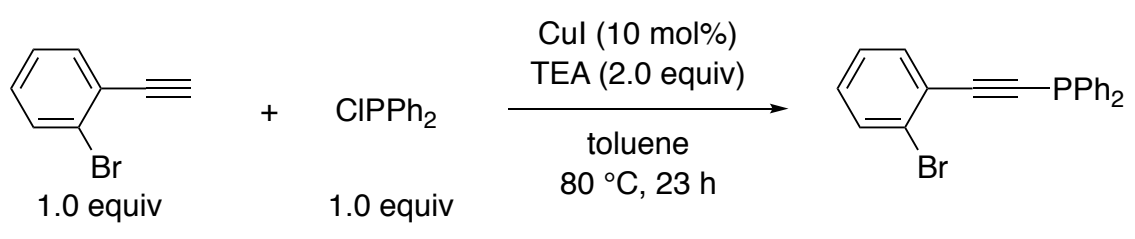

According to the protocol by Orita et al., ${ }^{12}$ 1-bromo-2-ethynylbenzene $(437.6 \mathrm{mg}, 2.42 \mathrm{mmol} .1 .0$ equiv) and $\mathrm{CuI}$ (46.0 $\mathrm{mg}, \quad 0.24 \mathrm{mmol}, \quad 10 \mathrm{~mol} \%)$ were added to toluene $(10 \mathrm{~mL})$. To the mixture were added chlorodiphenylphosphine $(533.3 \mathrm{mg}, 2.42 \mathrm{mmol}, 1.0$ equiv) and triethylamine (0.68 mL, $4.9 \mathrm{mmol}, 2.0$ equiv) successively, and the resultant mixture was stirred at $80^{\circ} \mathrm{C}$ for $23 \mathrm{~h}$. The reaction was cooled to rt and diluted with EtOAc, filtered through a pad of Celite. The filtrate was washed with $10 \% \mathrm{NH}_{3}$ aq., $\mathrm{H}_{2} \mathrm{O}$, then brine ( $\mathrm{x} 1 \mathrm{each}$ ), dried over $\mathrm{Na}_{2} \mathrm{SO}_{4}$, filtered, and concentrated in vacuo. Purification with MPLC (eluent: hexane/EtOAc) followed by GPC gave the desired product in $62 \%$ yield as yellow viscose oil. ${ }^{1} \mathbf{H}$ NMR $\left(400 \mathbf{~ M H z}, \mathbf{C D C l}_{3}\right): \delta .7 .19$ (apparent dt, $J=7.8$, $1.7 \mathrm{~Hz}, 1 \mathrm{H}), 7.27$ (apparent dt, $J=8.1,1.2 \mathrm{~Hz}, 1 \mathrm{H}), 7.31-7.39(\mathrm{~m}, 6 \mathrm{H}), 7.54(\mathrm{dd}, J=7.7,1.7 \mathrm{~Hz}, 1 \mathrm{H}), 7.60(\mathrm{dd}, J=8.0$, $1.0 \mathrm{~Hz}, 1 \mathrm{H}), 7.70-7.75(\mathrm{~m}, 4 \mathrm{H}) .{ }^{13} \mathbf{C}$ NMR $\left(\mathbf{1 0 0} \mathbf{~ M H z}, \mathbf{C D C l}_{3}\right): \delta 91.0\left(\mathrm{~d}, J_{\mathrm{C}-\mathrm{P}}=9.1 \mathrm{~Hz}\right), 105.7\left(\mathrm{~d}, J_{\mathrm{C}-\mathrm{p}}=3.7 \mathrm{~Hz}\right)$, $125.0(\mathrm{br}), 125.7\left(\mathrm{~d}, J_{\mathrm{C}-\mathrm{P}}=2.2 \mathrm{~Hz}\right), 127.0,128.6\left(\mathrm{~d}, J_{\mathrm{C}-\mathrm{P}}=7.6 \mathrm{~Hz}\right), 129.1,129.9,132.5,132.8\left(\mathrm{~d}, J_{\mathrm{C}-\mathrm{P}}=22.2 \mathrm{~Hz}\right), 133.7$ $\left(\mathrm{d}, J_{\mathrm{C}-\mathrm{P}}=1.2 \mathrm{~Hz}\right.$ ), 136.0 (d, $J_{\mathrm{C}-\mathrm{P}}=6.1 \mathrm{~Hz}$ ). IR (ATR-IR): 3067, 3054, 2167, 1584, 1477, 1465, 1434, 1258, 1223, 1183, 1119, 1095, 1046, 1027, 999, 913, 846, 749, 740, 693, 670, 630, 549, 509, 488, $460 \mathrm{~cm}^{-1}$. HRMS (ESI): $m / z$ calculated for $\mathrm{C}_{20} \mathrm{H}_{14} \mathrm{AgBrP}{ }^{+}[\mathrm{M}+\mathrm{Ag}]^{+}=470.9062$, found: 470.9054 .

\section{9-((2-bromophenyl)ethynyl)-9H-carbazole}

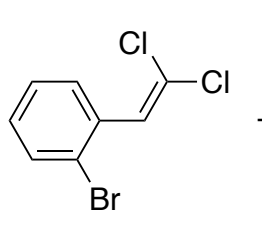

2.0 equiv<smiles>c1ccc2c(c1)[nH]c1ccccc12</smiles>

1.0 equiv

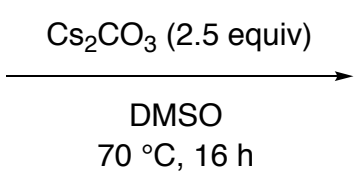

$70{ }^{\circ} \mathrm{C}, 16 \mathrm{~h}$

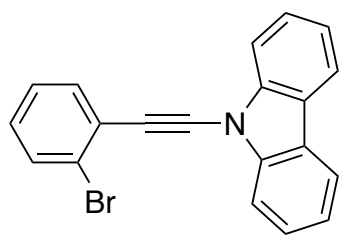

1-Bromo-2-(2,2-dichlorovinyl)benzene $(252 \mathrm{mg}, 1.0 \mathrm{mmol}, 2.0$ equiv) and carbazole ( $83.6 \mathrm{mg}, 0.5 \mathrm{mmol}, 1.0 \mathrm{equiv})$ were dissolved in DMSO $(2.5 \mathrm{~mL})$. To the mixture was then added $\mathrm{Cs}_{2} \mathrm{CO}_{3}(815 \mathrm{mg}, 2.5 \mathrm{mmol}, 5.0$ equiv), and the reaction was stirred at $70{ }^{\circ} \mathrm{C}$ for $16 \mathrm{~h}$. The reaction was quenched with cold water and extracted with $\mathrm{CH}_{2} \mathrm{Cl}_{2} \times 3$. The combined organic phase was washed with brine $\mathrm{x} 1$, dried over $\mathrm{Na}_{2} \mathrm{SO}_{4}$, filtered, and concentrated. Purification with MPLC (eluent: hexane) and recrystallization from hexane gave the desired ynamide in $46 \%$ yield $(79.2 \mathrm{mg})$ as off-white solid. ${ }^{1} \mathbf{H}$ NMR (500 $\left.\mathbf{~ M H z}, \mathbf{C D C l}_{3}\right): \delta 7.18(\mathrm{ddd}, J=7.8,1.8,0.6 \mathrm{~Hz}, 1 \mathrm{H}), 7.32-7.38(\mathrm{~m}, 3 \mathrm{H}), 7.55(\mathrm{ddd}, J=$

(12) Yang, X.; Matsuo, D.; Suzuma, Y.; Fang, J.-K.; Xu, F.; Orita, A.; Otera, J.; Kajiyama, S.; Koumura, N.; Hara, K. Synlett 201 1, 2402. 
8.2, 7.3, $1.2 \mathrm{~Hz}, 2 \mathrm{H}), 7.63(\mathrm{dd}, J=7.6,1.5 \mathrm{~Hz}, 1 \mathrm{H}), 7.67(\mathrm{dd}, J=8.1,1.2 \mathrm{~Hz}, 1 \mathrm{H}), 7.83(\mathrm{~d}, J=7.9 \mathrm{~Hz}, 2 \mathrm{H}), 8.04(\mathrm{~d}, J=$ $7.9 \mathrm{~Hz}, 2 \mathrm{H}) .{ }^{13} \mathbf{C}$ NMR (125 MHz, $\left.\mathbf{C D C l}_{3}\right): \delta$ 74.1, 83.8, 111.7, 120.6, 122.5, 123.9, 124.3, 125.6, 127.0, 127.3, 128.7, 132.3, 1326, 140.5. IR (ATR-IR): 3049, 2250, 1598, 1498, 1478, 1444, 1396, 1336, 1306, 1254, 1238, 1221, 1146, 1117, 1066, 1022, 941, 860, 743, 719, 655, 641, 615, 572, 560, 527, $506 \mathrm{~cm}^{-1}$. HRMS (ESI): $\mathrm{m} / z$ calculated for $\mathrm{C}_{20} \mathrm{H}_{12} \mathrm{AgBrN}^{+}[\mathrm{M}+\mathrm{Ag}]^{+}=451.9199$, found: 451.9186 .

\section{2-bromo-3-(phenylethynyl)naphthalene}

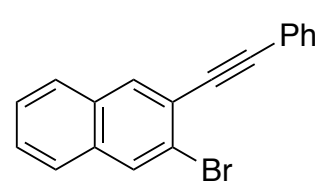

In a $20 \mathrm{~mL}$ Schlenk tube were suspended CuI (25.4 mg, $133 \mu \mathrm{mol}, 3.3 \mathrm{~mol} \%), \mathrm{PdCl}_{2}\left(\mathrm{PPh}_{3}\right)_{2}$ (56.2 $\mathrm{mg}, 80 \mu \mathrm{mol}, 2.0 \mathrm{~mol} \%$ ), and 2,3-dibromonaphthalene (1.14 g, $4.0 \mathrm{mmol}, 1.0$ equiv.) in a mixture of $\mathrm{Et}_{3} \mathrm{~N}(20 \mathrm{~mL})$ and THF ( $\left.2 \mathrm{~mL}\right)$. Phenylacetylene ( $409 \mathrm{mg}, 4.0 \mathrm{mmol}, 1.0$ equiv.) was added to the suspension and the resultant mixture was stirred for $37 \mathrm{~h}$ at $60^{\circ} \mathrm{C}$, cooled to rt and quenched with sat. $\mathrm{NH}_{4} \mathrm{Cl}$ aq. under vigorous stirring. Phases were separated and aqueous layer was extracted with EtOAc x 3, and combined organic phase was dried over $\mathrm{Na}_{2} \mathrm{SO}_{4}$, filtered, and concentrated to give brown oil. Purification with MPLC (eluent: hexane $\rightarrow$ hexane:EtOAc 80:20) followed by recycling preparative HPLC gave the desired product as yellow solid, which was recrystallized from hexane to give a white wool-like solid in $44 \%$ yield (542 mg). The ${ }^{13} \mathrm{C}$ NMR spectrum is in good agreement with the reported one. ${ }^{13}{ }^{1} \mathbf{H}$ NMR $\left(\mathbf{4 0 0} \mathbf{~ M H z}, \mathbf{C D C l}_{3}\right): \delta$ 7.37-7.41 (m, $3 \mathrm{H}), 7.50(\mathrm{dd}, J=6.8,3.2 \mathrm{~Hz}, 1 \mathrm{H}), 7.51(\mathrm{dd}, J=6.8,3.6 \mathrm{~Hz}, 1 \mathrm{H}), 7.61-7.64(\mathrm{~m}, 2 \mathrm{H}), 7.72-7.76(\mathrm{~m}, 1 \mathrm{H})$, 7.72-7.76 (m, 1H), 7.77-7.81 (m, 1H), $8.09(\mathrm{~s}, 1 \mathrm{H}), 8.12(\mathrm{~s}, 1 \mathrm{H}) .{ }^{13} \mathbf{C}$ NMR (100 MHz, $\left.\mathbf{C D C l}_{3}\right): \delta 88.4,93.6,122.7$, 123.0, 126.7, 127.5, 127.6, 128.4 (overlapped), 128.7, 131.0, 131.7, 131.8, 133.0, 133.6.

\section{1-bromo-4,5-difluoro-2-(phenylethynyl)benzene}

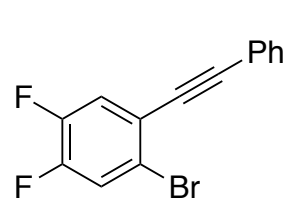

In a $20 \mathrm{~mL}$ Schlenk tube were suspended 1,2-dibromo-4,5-difluorobenzene $(1.09 \mathrm{~g}, 4.0 \mathrm{mmol}$, 1.0 equiv), $\mathrm{CuI}(25.4 \mathrm{mg}, 133 \mu \mathrm{mol}, 3.3 \mathrm{~mol} \%), \mathrm{PdCl}_{2}\left(\mathrm{PPh}_{3}\right)_{2}(56.2 \mathrm{mg}, 80 \mu \mathrm{mol}, 2.0 \mathrm{~mol} \%)$ in $\mathrm{Et}_{3} \mathrm{~N}(20 \mathrm{~mL})$. Phenylacetylene ( $409 \mathrm{mg}, 4.0 \mathrm{mmol}, 1.0$ equiv) was added to the suspension and the resultant mixture was stirred for $39 \mathrm{~h}$ at $70{ }^{\circ} \mathrm{C}$, cooled to rt and quenched with sat. $\mathrm{NH}_{4} \mathrm{Cl}$ aq. under vigorous stirring. Phases were separated and aqueous layer was extracted with EtOAc $x 3$, and combined organic phase was dried over $\mathrm{Na}_{2} \mathrm{SO}_{4}$, filtered, and concentrated to give a brown oil. Purification with MPLC (eluent: hexane) gave the desired product as a white solid in $46 \%$ yield $(542 \mathrm{mg}) \cdot{ }^{1} \mathrm{H}$ and ${ }^{13} \mathrm{C}$ NMR spectra are in agreement with the precedented report. ${ }^{14}{ }^{1} \mathbf{H}$ NMR (400 $\left.\mathbf{~ M H z}, \mathbf{C D C l}_{3}\right): \delta$ 7.35-7.40 (m, 4H), $7.44(\mathrm{dd}, J=9.6,2.1 \mathrm{~Hz}, 1 \mathrm{H}), 7.55-7.57(\mathrm{~m}$, 2H). ${ }^{13} \mathbf{C}$ NMR (100 MHz, $\left.\mathbf{C D C l}_{3}\right): \delta 86.2(\mathrm{t}, J=2.7 \mathrm{~Hz}), 94.5(\mathrm{~d}, J=2.0 \mathrm{~Hz}), 120.0(\mathrm{dd}, J=7.0,3.7 \mathrm{~Hz}), 121.2(\mathrm{~d}, J$ $=19.3 \mathrm{~Hz}), 121.6(\mathrm{~d}, J=20.4 \mathrm{~Hz}), 122.2(\mathrm{dd}, J=7.5,4.2 \mathrm{~Hz}), 122.3,128.5,129.0,131.7,148.4(\mathrm{dd}, J=66.4,13.1 \mathrm{~Hz})$, $150.8(\mathrm{dd}, J=72.3,13.1 \mathrm{~Hz}) .{ }^{19} \mathbf{F}$ NMR $\left(376 \mathbf{~ M H z}, \mathbf{C D C l}_{3}\right): \delta-138.1--138.0(\mathrm{~m}, 1 \mathrm{~F}),-133.1--133.0(\mathrm{~m}, 1 \mathrm{~F})$.

\section{5-bromo-6-(phenylethynyl)benzo $[d][1,3]$ dioxole}

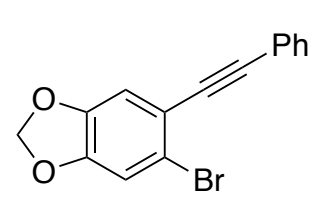

In a $20 \mathrm{~mL}$ Schlenk tube were suspended 5,6-dibromobenzo[d][1,3] dioxole $(560 \mathrm{mg}, 2.0$ mmol, 1.0 equiv), $\mathrm{CuI}(12.7 \mathrm{mg}, 66.6 \mu \mathrm{mol}, 3.3 \mathrm{~mol} \%), \mathrm{PdCl}_{2}\left(\mathrm{PPh}_{3}\right)_{2}(28.1 \mathrm{mg}, 40 \mu \mathrm{mol}, 2.0$ $\mathrm{mol} \%)$ in $\mathrm{Et}_{3} \mathrm{~N}(10 \mathrm{~mL})$. Phenylacetylene $(204 \mathrm{mg}, 2.0 \mathrm{mmol}, 1.0$ equiv) was added to the suspension and the resultant mixture was stirred for $16 \mathrm{~h}$ at $70{ }^{\circ} \mathrm{C}$, cooled to $\mathrm{rt}$, and volatiles were evaporated in vacuo. The crude residue was pre-adsorbed on $\mathrm{SiO}_{2}$ and purified with MPLC (eluent: hexane $\rightarrow$ hexane:EtOAc 80:20) followed by recycling preparative HPLC gave the desired product as off-white solid in $23 \%$ yield

(13) Kawase, T.; Fujiwara, T.; Kitamura, C.; Konishi, A.; Hirano, Y.; Matsumoto, K.; Kurata, H.; Kubo, T.; Shinamura, S.; Mori, H.; Miyazaki, E.; Takimiya, K. Angew. Chem. Int. Ed., 2010, 49, 7728.

(14) Mule, R. D.; Shaikh, A. C.; Gade, A. B.; Patil, N. T. Chem. Commun. 2018, 54, 11909. 
(138.8 mg). ${ }^{1} \mathbf{H}$ NMR (400 MHz, $\left.\mathbf{C D C l}_{3}\right): \delta 6.00(\mathrm{~s}, 2 \mathrm{H}), 6.99(\mathrm{~s}, 1 \mathrm{H}), 7.06(\mathrm{~s}, 1 \mathrm{H})$, 7.33-7.36 (m, 3H), 7.54-7.56 (m, 2H). ${ }^{13} \mathbf{C}$ NMR (100 MHz, $\mathbf{C D C l}_{3}$ ): $\delta 88.1,92.4,102.1,112.1,112.7,117.8,118.1,123.0,128.3,128.4,131.5,147.0$, 148.5. IR (ATR-IR): 2898, 1597, 1502, 1493, 1471, 1442, 1415, 1396, 1338, 1274, 1228, 1210, 1148, 1099, 1069, 1037, 969, 933, 860, 836, 755, 689, 654, 580, 527, $491 \mathrm{~cm}^{-1}$. HRMS (APCI): $m / z$ calculated for $\mathrm{C}_{15} \mathrm{H}_{10} \mathrm{BrO}_{2}^{+}[\mathrm{M}+\mathrm{H}]^{+}=300.9859$, found: 300.9854 .

\section{5-bromo-1,3-dihexyl-6-(phenylethynyl)-1,3-dihydro-2H-benzo[d]imidazol-2-one}

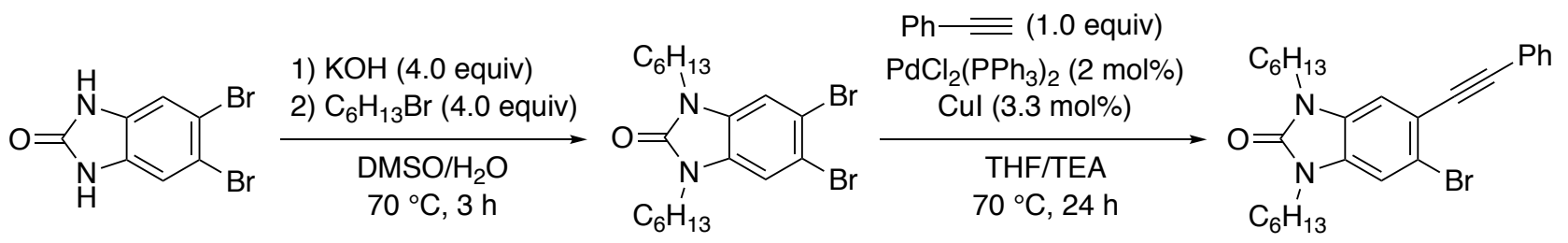

To a solution of 5,6-dibromo-1,3-dihydro-2H-benzo[d]imidazol-2-one ${ }^{15}$ (1.75 g, $6.0 \mathrm{mmol}$, 1.0 equiv) in DMSO (35 $\mathrm{mL}$ ) was added a solution of $\mathrm{KOH}\left(1.36 \mathrm{~g}, 24.2 \mathrm{mmol}, 4.04\right.$ equiv) in $\mathrm{H}_{2} \mathrm{O}(18 \mathrm{~mL})$ carefully at $\mathrm{rt}$ (exothermic!). The mixture was stirred at $\mathrm{rt}$ for $10 \mathrm{~min}$, then 1-bromohexane $(3.96 \mathrm{~g}, 24.0 \mathrm{mmol}, 4.0$ equiv) was added. The resultant mixture was stirred at $70{ }^{\circ} \mathrm{C}$ for $3 \mathrm{~h}$. After cooled to rt, the reaction was acidified with $1 \mathrm{H} \mathrm{HCl}$ aq., extracted with EtOAc x 3. The combined organic phase was washed with $\mathrm{H}_{2} \mathrm{O}$ × 5, brine $\times 1$, dried over $\mathrm{Na}_{2} \mathrm{SO}_{4}$, filtered, and concentrated to give brown oil, which was purified with MPLC (eluent: hexane $\rightarrow$ hexane/EtOAc 9:1) to give the desired product, 5,6-dibromo-1,3-dihexyl-1,3-dihydro-2H-benzo[d]imidazol-2-one, in 68\% yield (1.89 g). ${ }^{1} \mathbf{H}$ NMR $\left(400 \mathrm{MHz}, \mathrm{CDCl}_{3}\right): \delta .0 .88(\mathrm{t}, J=6.9 \mathrm{~Hz}, 6 \mathrm{H}), 1.27-1.36(\mathrm{~m}, 12 \mathrm{H}), 1.67-1.74(\mathrm{~m}, 4 \mathrm{H}), 3.81(\mathrm{t}, J=7.4 \mathrm{~Hz}, 4 \mathrm{H}), 7.20$ (s, 2H). ${ }^{13} \mathbf{C}$ NMR (100 MHz, $\mathbf{C D C l}_{3}$ ): $\delta$ 14.0, 22.5, 26.4, 28.2, 31.4, 41.4, 112.0, 115.5, 129.9, 153.9. IR (ATR-IR): 2955, 2927, 2857, 1705, 1617, 1591, 1497, 1457, 1401, 1372, 1307, 1226, 1182, 1115, 1089, 913, 876, 846, 747, 725, 641, $602 \mathrm{~cm}^{-1}$. To a suspension of 5,6-dibromo-1,3-dihexyl-1,3-dihydro-2H-benzo[d]imidazol-2-one (921 mg, 2.0 mmol, 1.0 equiv), $\mathrm{CuI}(12.7 \mathrm{mg}, 67 \mu \mathrm{mol}, 3.3 \mathrm{~mol} \%)$, and $\mathrm{PdCl}_{2}\left(\mathrm{PPh}_{3}\right)_{2}(28.1 \mathrm{mg}, 40 \mu \mathrm{mol}, 2.0 \mathrm{~mol} \%)$ in a mixture of triethylamine $(5 \mathrm{~mL})$ and THF $(5 \mathrm{~mL})$ was added phenylacetylene $(204 \mathrm{mg}, 2.0 \mathrm{mmol}, 1.0$ equiv) was added and the resultant mixture was stirred at $70^{\circ} \mathrm{C}$ for $24 \mathrm{~h}$. The reaction was then cooled to rt and quenched with sat. $\mathrm{NH}_{4} \mathrm{Cl}$ aq. and extracted with EtOAc $\times 3$. The combined organic phase was dried over $\mathrm{Na}_{2} \mathrm{SO}_{4}$, filtered, and concentrated to give brown oil. Purification with MPLC (eluent: hexane $\rightarrow$ hexane/EtOAc 97:3) gave the desired product as yellow viscose oil in 29\% yield (282 mg). ${ }^{1} \mathbf{H}$ NMR (400 $\left.\mathbf{~ M H z}, \mathbf{C D C l}_{3}\right): \delta .0 .880(\mathrm{t}, J=7.0 \mathrm{~Hz}, 3 \mathrm{H}), 0.883(\mathrm{t}, J=7.0 \mathrm{~Hz}, 3 \mathrm{H})$, 1.30-1.37 (m, 12H), 1.69-1.77 (m, 4H), $3.83(\mathrm{t}, J=7.2 \mathrm{~Hz}, 2 \mathrm{H}), 3.85(\mathrm{t}, J=7.2 \mathrm{~Hz}, 2 \mathrm{H}), 7.15(\mathrm{~s}, 1 \mathrm{H}), 7.20(\mathrm{~s}, 1 \mathrm{H})$, 7.33-7.39 (m, 3H), 7.56-7.61 (m, 2H). ${ }^{13} \mathbf{C}$ NMR (100 $\left.\mathbf{M H z}, \mathbf{C D C l}_{3}\right): \delta 14.0$ (2C, overlapped), 22.5 (2C, overlapped), 26.44, 26.45, 28.23, 28.28, 31.39, 31.41, 41.36, 41.41, 88.7, 92.5, 111.3, 111.4, 117.2, 117.7, 123.1, 128.40, 128.43, 128.8, 130.6, 131.6, 154.1. IR (ATR-IR): 2954, 2927, 2857, 1708, 1622, 1597, 1585, 1497, 1456, 1404, 1373, 1305, 1225, 1183, 1026, 966, 913, 853, 754, 725, 690, 640, $558 \mathrm{~cm}^{-1}$. HRMS (ESI): $\mathrm{m} / z$ calculated for $\mathrm{C}_{27} \mathrm{H}_{33} \mathrm{BrN}_{2} \mathrm{NaO}^{+}[\mathrm{M}+\mathrm{Na}]^{+}=503.1668$, found: 503.1652 .

\section{3-bromo-5-methyl-2-(phenylethynyl)pyridine}

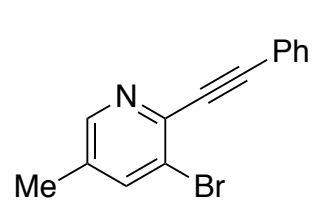

In a $20 \mathrm{~mL}$ Schlenk tube were suspended 2,3-dibromo-5-methylpyridine $(502 \mathrm{mg}, 2.0 \mathrm{mmol}$, 1.0 equiv), $\mathrm{CuI}(12.7 \mathrm{mg}, 66.6 \mu \mathrm{mol}, 3.3 \mathrm{~mol} \%), \mathrm{PdCl}_{2}\left(\mathrm{PPh}_{3}\right)_{2}(28.1 \mathrm{mg}, 40 \mu \mathrm{mol}, 2.0 \mathrm{~mol} \%)$ in $\mathrm{Et}_{3} \mathrm{~N}$ (10 mL). Phenylacetylene (204 mg, $2.0 \mathrm{mmol}, 1.0$ equiv) was added to the suspension and the resultant mixture was stirred for $23 \mathrm{~h}$ at $70{ }^{\circ} \mathrm{C}$, cooled to rt and quenched with sat.

( 15 ) Ramadoss, V.; Alonso-Castro, A. J.; Campos-Xolalpa, N.; Ortiz-Alvarado, R.; Yahuaca-Juárez, B.; Solorio-Alvarado, C. R. RSC Adv. 2018, 8, 20761. 
$\mathrm{NH}_{4} \mathrm{Cl}$ aq. under vigorous stirring. Phases were separated and aqueous layer was extracted with EtOAc $\mathrm{x} 3$, and combined organic phase was dried over $\mathrm{Na}_{2} \mathrm{SO}_{4}$, filtered, and concentrated to give brown oil. Purification with MPLC (eluent: hexane/EtOAc 88:12 $\rightarrow$ 67:33) gave the desired product as off-white solid, which was recrystallized from hexane to give off-white solid in 75\% yield (410.4 mg). ${ }^{1} \mathbf{H}$ NMR (400 $\left.\mathbf{~ M H z}, \mathbf{C D C l}_{3}\right): \delta 2.35$ (s, 3H), 7.35-7.38 (m, 3H), 7.63-7.65 (m, 2H), 7.75 (s, 1H), 8.37 (s, 1H). ${ }^{13} \mathbf{C}$ NMR (100 MHz, $\left.\mathbf{C D C l}_{3}\right): \delta$ 18.0, 87.4, 93.1, 122.1, 123.3, 128.4, 129.1, 132.1, 134.1, 140.1, 140.8, 148.9. IR (ATR-IR): 3046, 2916, 2224, 2204, 1596, 1580, 1534, 1491, 1443, 1384, 1316, 1227, 1200, 1160, 1069, 1049, 1023, 1000, 919, 884, 818, 756, 741, 689, 559, 527, $504 \mathrm{~cm}^{-1}$. HRMS (ESI): $m / z$ calculated for $\mathrm{C}_{14} \mathrm{H}_{10} \mathrm{BrNNa}^{+}[\mathrm{M}+\mathrm{Na}]^{+}=293.9889$, found: 293.9890 .

\section{3-bromo-2-(phenylethynyl)benzo[b]thiophene}

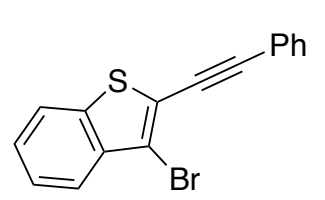

In a $20 \mathrm{~mL}$ Schlenk tube were suspended 2,3-dibromobenzo[b] thiophene $(204 \mathrm{mg}, 2.0 \mathrm{mmol}$, 1.0 equiv), $\mathrm{CuI}$ (12.7 mg, $66.6 \mu \mathrm{mol}, 3.3 \mathrm{~mol} \%), \mathrm{PdCl}_{2}\left(\mathrm{PPh}_{3}\right)_{2}(28.1 \mathrm{mg}, 40 \mu \mathrm{mol}, 2.0 \mathrm{~mol} \%)$ in $\mathrm{Et}_{3} \mathrm{~N}(10 \mathrm{~mL})$. Phenylacetylene (204 mg, $2.0 \mathrm{mmol}, 1.0$ equiv) was added to the suspension and the resultant mixture was stirred for $28 \mathrm{~h}$ at $70^{\circ} \mathrm{C}$, cooled to rt and volatiles were removed in vacuo. The crude residue was pre-adsorbed on $\mathrm{SiO}_{2}$ and purified with MPLC (eluent: hexane) followed by with recycling preparative HPLC gave the desired product as off-white solid in $63 \%$ yield $(396.9 \mathrm{mg}) .{ }^{1} \mathrm{H}$ and ${ }^{13} \mathrm{C}$ NMR spectra are in good agreement with the precedented report. ${ }^{16}{ }^{1} \mathbf{H}$ NMR $\left(400 \mathbf{~ M H z}, \mathbf{C D C l}_{3}\right): \delta$ 7.38-7.41 (m, 3H), 7.42-7.49 (m, 2H), 7.61-7.63 (m, 2H), 7.75-7.77 (m, 1H), 7.79-7.81 (m, $1 \mathrm{H}) .{ }^{13} \mathbf{C}$ NMR (100 MHz, $\left.\mathbf{C D C l}_{3}\right): \delta 81.7$, 99.1, 113.7, 120.4, 122.2, 122.3, 123.6, 125.5, 126.5, 128.5, 129.1, 131.7, 137.4, 138.2.

\section{((2-bromocyclohex-1-en-1-yl)ethynyl)benzene}
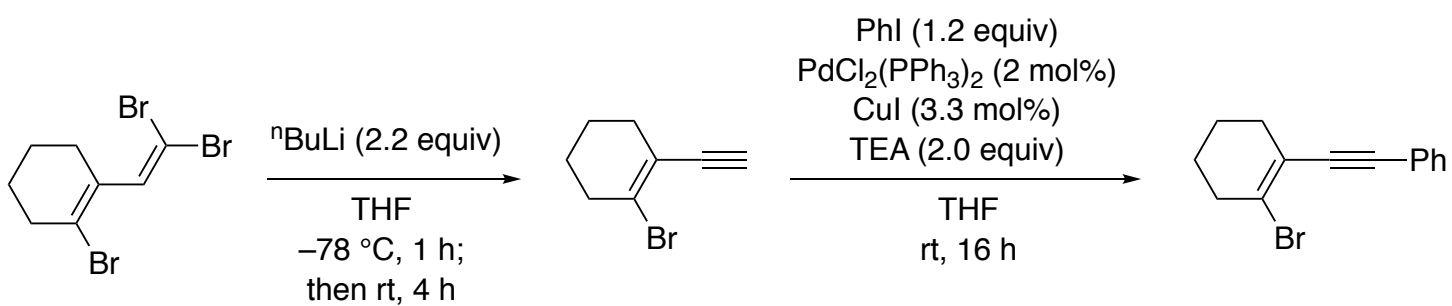

1-Bromo-2-(2,2-dibromovinyl)cyclohex-1-ene $(2.59 \mathrm{~g}, 7.51 \mathrm{mmol}, 1.0$ equiv) prepared according to the report by Horino et al. ${ }^{17}$ was dissolved in THF $(23 \mathrm{~mL})$. To the solution was added ${ }^{\mathrm{n}} \mathrm{BuLi}(2.72 \mathrm{M}$ in hexane, $6.07 \mathrm{~mL}, 16.5$ mmol, 2.2 equiv) dropwise at $-78{ }^{\circ} \mathrm{C}$ and stirred at the same temperature for $1 \mathrm{~h}$ and subsequently at rt for $4 \mathrm{~h}$. The reaction was quenched with sat. $\mathrm{NH}_{4} \mathrm{Cl}$ aq. and extracted with hexane $\mathrm{x} 3$, and the combined organic phase was dried over $\mathrm{Na}_{2} \mathrm{SO}_{4}$, filtered, and concentrated to give brown oil. Purification with MPLC (eluent: hexane) gave the desired product, 1-bromo-2-ethynylcyclohex-1-ene, as yellowish oil in $66 \%$ yield $(912.9 \mathrm{mg})$, which was used immediately for the next step. To a suspension of $\mathrm{CuI}(25.4 \mathrm{mg}, 0.13 \mathrm{mmol}, 3.3 \mathrm{~mol} \%), \mathrm{PdCl}_{2}\left(\mathrm{PPh}_{3}\right)_{2}(56.2 \mathrm{mg}, 0.080 \mathrm{mmol}, 2.0$ mol\%) in a mixture of triethylamine $(10 \mathrm{~mL})$ and THF $(10 \mathrm{~mL})$ were added iodobenzene $(979 \mathrm{mg}, 4.8 \mathrm{mmol}, 1.2$ equiv) and 1-bromo-2-ethynylcyclohex-1-ene $(740 \mathrm{mg}, 4.00 \mathrm{mg}, 1.0$ equiv). The resultant mixture was stirred at $\mathrm{rt}$ for $16 \mathrm{~h}$, and then quenched with sat. $\mathrm{NH}_{4} \mathrm{Cl}$ aq. and extracted with EtOAc $\times 3$. The combined organic phase was dried over $\mathrm{Na}_{2} \mathrm{SO}_{4}$, filtered, and concentrated to give brown oil. Purification with MPLC (eluent: hexane) gave the desired product as yellow oil in 72\% yield (891.1 mg). ${ }^{1} \mathbf{H}$ NMR (400 $\left.\mathbf{~ M H z , ~} \mathbf{C D C l}_{3}\right): \delta 1.67-1.77(\mathrm{~m}, 4 \mathrm{H}), 2.34-2.38(\mathrm{~m}, 2 \mathrm{H})$, 2.58-2.62 (m, 2H), 7.29-7.32 (m, 3H), 7.46-7.49 (m, 2H). ${ }^{13} \mathbf{C}$ NMR (100 MHz, $\left.\mathbf{C D C l}_{3}\right): \delta 21.8,24.1,31.8,36.3,89.6$, 92.8, 121.3, 123.3, 128.26, 128.29, 129.0, 131.6. IR (ATR-IR): 2933, 2882, 2860, 2837, 1621, 1594, 1571, 1489, 1442,

(16) Lyaskovskyy, V.; Fröhlich, R.; Würthwein, E.-U. Chem. Eur. J. 2007, 13, 3113.

(17) Horino Y.; Nakashima, Y.; Hashimoto, K.; Kuroda, S. Synlett 2010, 2879. 
$1436,1332,1260,1240,1173,1137,1097,1069,1026,978,913,828,816,754,689,609,575,544,525,509,496 \mathrm{~cm}^{-1}$. HRMS (APCI): $m / z$ calculated for $\mathrm{C}_{14} \mathrm{H}_{14} \mathrm{Br}^{+}[\mathrm{M}+\mathrm{H}]^{+}=261.0273$, found: 261.0272 .

7-bromo-1-((4-(trifluoromethyl)phenyl)ethynyl)-1H-indole

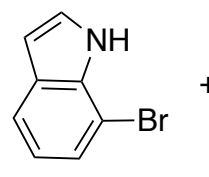

1.0 equiv<smiles>FC(F)(F)c1ccc(C=C(Cl)Cl)cc1</smiles>

2.0 equiv

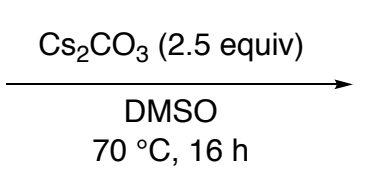

$70{ }^{\circ} \mathrm{C}, 16 \mathrm{~h}$

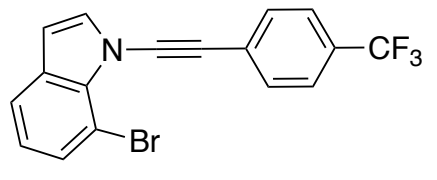

1-(2,2-dichlorovinyl)-4-(trifluoromethyl)benzene ( $482 \mathrm{mg}, 2.0 \mathrm{mmol}, 2.0$ equiv) and 7-bromo- $1 \mathrm{H}$-indole (196 mg, $1.0 \mathrm{mmol}, 1.0$ equiv) were dissolved in DMSO $(5 \mathrm{~mL})$. To the mixture was then added $\mathrm{Cs}_{2} \mathrm{CO}_{3}(1.63 \mathrm{~g}, 5.0 \mathrm{mmol}, 5.0$ equiv), and the reaction was stirred at $70{ }^{\circ} \mathrm{C}$ for $16 \mathrm{~h}$. The reaction was quenched with cold water and extracted with $\mathrm{Et}_{2} \mathrm{O} \times 3$. The combined organic phase was washed with water $\times 3$, brine $\times 1$, dried over $\mathrm{Na}_{2} \mathrm{SO}_{4}$, filtered, and concentrated. Purification with MPLC (eluent: hexane) gave the desired ynamide in $65 \%$ yield as off-white solid. ${ }^{1} \mathbf{H}$ NMR $\left(400 \mathrm{MHz}, \mathbf{C D C l}_{3}\right): \delta 6.60(\mathrm{~d}, J=3.4 \mathrm{~Hz}, 1 \mathrm{H}), 7.04(\mathrm{dd}, J=7.8,7.6 \mathrm{~Hz}, 1 \mathrm{H}), 7.25(\mathrm{~d}, J=3.4 \mathrm{~Hz}, 1 \mathrm{H}), 7.45(\mathrm{dd}$, $J=7.7,0.7 \mathrm{~Hz}, 1 \mathrm{H}), 7.52(\mathrm{dd}, J=7.8,0.7 \mathrm{~Hz}, 1 \mathrm{H}), 7.59(\mathrm{~s}, 4 \mathrm{H}) .{ }^{13} \mathbf{C} \mathbf{~ N M R}\left(\mathbf{1 0 0} \mathbf{~ M H z}, \mathbf{C D C l}_{3}\right): \delta 71.2,84.3,105.9$, 106.6, 121.0, 123.6, 125.5, 127.02, 127.03, 128.9, $129.6(\mathrm{q}, J=33.0 \mathrm{~Hz}), 130.57,130.62,131.7,133.8 .{ }^{19} \mathbf{F}$ NMR $(376$ MHz, $\mathbf{C D C l}_{3}$ ): $\delta$-62.6. IR (ATR-IR): 2249, 1613, 1566, 1526, 1474, 1437, 1411, 1386, 1318, 1255, 1242, 1188, 1159, 1138, 1105, 1065, 1034, 1014, 964, 919, 897, 836, 814, 788, 753, 718, 599, $512 \mathrm{~cm}^{-1}$. HRMS (ESI): $m / z$ calculated for $\mathrm{C}_{17} \mathrm{H}_{9} \mathrm{AgBrF}_{3} \mathrm{~N}^{+}[\mathrm{M}+\mathrm{Ag}]^{+}=491.8916$, found: 491.8915 . 


\section{2-3. Hydrosilylation Leading to Diverse Benzosiloles (Table 2)}

General Procedure: 5-((1,2-diphenyl-1H-benzo[b]silol-1-yl)oxy)-2,5-dimethylhexan-2-ol (3a)

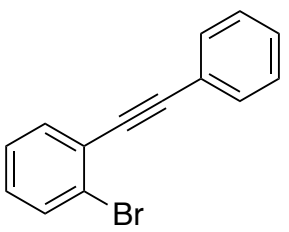

1) ${ }^{\mathrm{t}} \mathrm{BuLi}\left(2.0\right.$ equiv), $-78^{\circ} \mathrm{C}, 15 \mathrm{~min}$

2) $1 \mathrm{a}$ ( 2.0 equiv), $-78^{\circ} \mathrm{C}, 15 \mathrm{~min}$ ; then rt, $1 \mathrm{~h}$

$\operatorname{THF}(0.04 \mathrm{M})$
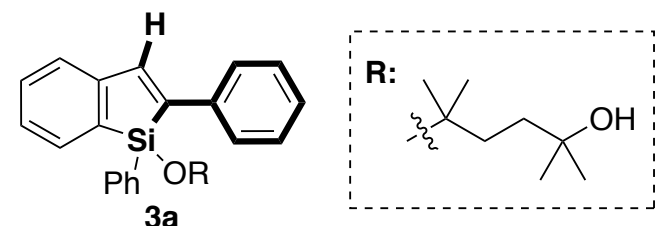

1-Bromo-2-(phenylethynyl)benzene $\mathbf{2 a}(77.1 \mathrm{mg}, 0.30 \mathrm{mmol}, 1.0$ equiv) and anhydrous THF (7.5 mL) were added to a heat gun-dried Schlenk tube. To the tube was added ${ }^{t} \mathrm{BuLi}$ (1.48 $\mathrm{M}$ in ${ }^{\mathrm{n}}$ pentane solution, $0.60 \mathrm{mmol}, 2.0$ equiv) slowly at $-78{ }^{\circ} \mathrm{C}$ and the mixture was stirred for $15 \mathrm{~min}$ before $1 \mathrm{a}(150 \mathrm{mg}, 0.60 \mathrm{mmol}, 2.0$ equiv) was added. After stirring for $15 \mathrm{~min}$ at $-78^{\circ} \mathrm{C}$, the resultant mixture was warmed to rt in water bath and stirred for $1 \mathrm{~h}$. The reaction was quenched with sat. $\mathrm{NH}_{4} \mathrm{Cl}$ aq., followed by extraction with EtOAc $\mathrm{x}$ 3. The combined organic layer was dried over $\mathrm{Na}_{2} \mathrm{SO}_{4}$, filtered and concentrated in vacuo. Purification with MPLC (eluent: hexane/EtOAc 4:1) afforded the product 3a as colorless oil in 93\% yield (119.2 mg). ${ }^{1} \mathbf{H}$ NMR (500 $\left.\mathbf{~ M H z}, \mathbf{C D C l}_{3}\right): \delta 1.03$ (s, 1H), 1.10 (s, 3H), 1.11(s, 3H), 1.18 (s, 3H), 1.23 (s, 3H), 1.49-1.61 (m, 4H), 7.19 (dd, J = 7.3, 1.2 Hz, 2H), 7.26-7.32 (m, 5H), 7.34-7.39 (m, 2H), 7.51-7.54 (m, 3H), $7.64(\mathrm{dd}, J=6.7,1.2 \mathrm{~Hz}, 2 \mathrm{H}), 7.65(\mathrm{~s}, 1 \mathrm{H}) .{ }^{13} \mathbf{C} \mathbf{N M R}\left(125 \mathbf{M H z}, \mathbf{C D C l}_{3}\right): \delta 29.25(2 \mathrm{C}$, overlapped), 29.32, 29.7, 38.4, 39.0, 70.9, 76.5, 124.8, 127.2, 127.4, 127.7, 128.2, 128.7, 130.2, 131.0, 132.8, 134.1, 134.4, 135.7, 138.4, 142.2, 143.2, 147.9. ${ }^{29}$ Si NMR (79.5 MHz, $\mathbf{C D C l}_{3}$ ): -16.0. IR (ATR-IR): 3400, 2970, 1429, 1367, 1219, 1103, 1064, 1021, 946, 899, 844, 765, 742, 718, 696, 600, 563, 491, 446, 432, 419, $405 \mathrm{~cm}^{-1}$. HRMS (ESI): $\mathrm{m} / z$ calculated for $\mathrm{C}_{28} \mathrm{H}_{32} \mathrm{O}_{2} \mathrm{Si}[\mathrm{M}+\mathrm{Na}]^{+}=451.2064$, found: 451.2065 .

\section{Large Scale Reaction:}

To a heat gun-dried 2-necked $200 \mathrm{~mL}$ egg-plant-shaped flask were added 2a (771.4 mg, $3.0 \mathrm{mmol}, 1.0$ equiv) and anhydrous THF $(75 \mathrm{~mL})$. The flask was cooled to $-78{ }^{\circ} \mathrm{C}$ (dry ice-acetone bath) and $t$-butyllithium $(4.05 \mathrm{~mL}, 6.0$ mmol; $1.48 \mathrm{M}$ nentane solution, 2.0 equiv) was slowly added over $5 \mathrm{~min}$. The mixture was stirred for $15 \mathrm{~min}$ at $-78^{\circ} \mathrm{C}$ and then $1 \mathrm{a}\left(1.50 \mathrm{~g}, 6.0 \mathrm{mmol}, 2.0\right.$ equiv) was added at $-78^{\circ} \mathrm{C}$. After stirring for $15 \mathrm{~min}$, the reaction mixture was allowed to warm to $70{ }^{\circ} \mathrm{C}$ and stirred for $1 \mathrm{~h}$. The reaction was quenched with sat. $\mathrm{NH}_{4} \mathrm{Cl}$ aq. (100 mL) and diluted with EtOAc $(30 \mathrm{~mL})$. The phases were separated and the aqueous layer was extracted with EtOAc $(50 \mathrm{~mL} \times 3)$. The combined organic layer was dried over $\mathrm{Na}_{2} \mathrm{SO}_{4}$, filtered, and concentrated under reduced pressure to give a crude mixture as colorless liquid. This crude mixture was purified with MPLC (eluent: hexane/EtOAc 4:1) to give 3a in 88\% yield $(1.13 \mathrm{~g})$.

For the Product of Entry 2, Table 1:

\section{4-((1,2-diphenyl-1H-benzo[b] silol-1-yl)oxy)-2,4-dimethylpentan-2-ol (3a')}

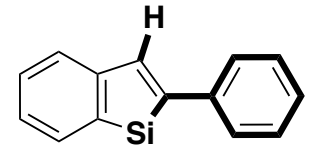

Ph' 'OR

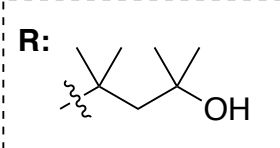

Following the General Procedure (1b, 1.0 equiv), the titled compound was obtained as colorless oil in 49\% yield (60.9 mg). ${ }^{1} \mathbf{H}$ NMR (400 $\left.\mathbf{M H z}, \mathbf{C D C l}_{3}\right): \delta 1.27$ (s, 6H), 1.34 (s, $3 \mathrm{H}), 1.36$ (s, 3H), 1.79 (s, 2H), 3.96 (brs, $1 \mathrm{H}), 7.20-7.24$ (m, 2H), 7.27-7.39 (m, 7H), 7.54-7.58 (m, 3H) 7.64 (dd, $J=7.8,1.5 \mathrm{~Hz}, 2 \mathrm{H}) 7.68$ (s, 1H). ${ }^{13} \mathbf{C}$ NMR (125 MHz, CDCl $\mathbf{~ M}^{2}$ : $\delta 31.67,31.68,31.71,31.8,54.3,71.4,79.1,125.0,127.2,127.5,127.9$, 128.4, 128.8, 130.6, 131.2, 133.0, 134.2, 135.0, 138.0, 142.5, 142.6, 147.6. IR (ATR-IR): 3534, 2970, 1590, 1439, $1429,1368,1164,1113,1063,991,945,900,826,765,735,716,696,652,599,558 \mathrm{~cm}^{-1}$. HRMS (ESI): $m / z$ calculated for $\mathrm{C}_{27} \mathrm{H}_{30} \mathrm{NaO}_{2} \mathrm{Si}[\mathrm{M}+\mathrm{Na}]^{+}=437.1907$, found: 437.1911 . 
5-((2-(4-chlorophenyl)-1-phenyl-1 H-benzo[b] silol-1-yl)oxy)-2,5-dimethylhexan-2-ol (3b)

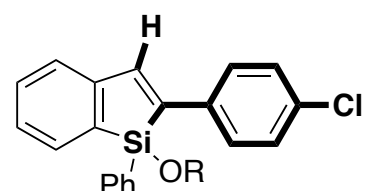

Following the General Procedure, the titled compound was obtained as colorless oil in 88\% yield (122.5 mg). ${ }^{1} \mathbf{H}$ NMR ( $\left.\mathbf{5 0 0} \mathbf{~ M H z}, \mathbf{C D C l}_{3}\right): \delta 1.08($ brs, $1 \mathrm{H}), 1.13(\mathrm{~s}, 3 \mathrm{H}), 1.14$ $(\mathrm{s}, 3 \mathrm{H}), 1.18(\mathrm{~s}, 3 \mathrm{H}), 1.22(\mathrm{~s}, 3 \mathrm{H}), 1.50-1.62(\mathrm{~m}, 4 \mathrm{H}), 7.20(\mathrm{dt}, J=7.2,0.9 \mathrm{~Hz}, 1 \mathrm{H}), 7.24$ $(\mathrm{dt}, J=8.9,2.1 \mathrm{~Hz}, 2 \mathrm{H}), 7.28(\mathrm{~d}, J=7.3 \mathrm{~Hz}, 1 \mathrm{H}), 7.29-7.32(\mathrm{~m}, 2 \mathrm{H}), 7.35-7.39(\mathrm{~m}, 2 \mathrm{H})$, $7.45(\mathrm{dt} J=8.5,2.1 \mathrm{~Hz}, 2 \mathrm{H}), 7.52(\mathrm{~d}, J=7.0 \mathrm{~Hz}, 1 \mathrm{H}), 7.61(\mathrm{dd}, J=8.2,0.9 \mathrm{~Hz}, 2 \mathrm{H}), 7.62(\mathrm{~s}, 1 \mathrm{H}) .{ }^{13} \mathbf{C}$ NMR $(125$ MHz, $\left.\mathbf{C D C l}_{3}\right): \delta 29.25,29.32,29.4,29.6,38.4,39.1,70.9,76.6,125.0,127.9,128.2,128.3,128.9,130.4,131.1,132.9$, 133.0, 133.7, 134.3, 135.5, 136.9, 141.9, 142.5, 147.7. IR (ATR-IR): 3435, 2971, 1970, 1587, 1537, 1487, 1440, 1429, $1367,1276,1218,1103,1064,1037,996,904,824,756,725,714,698,665,591,560,503,470,457,418,407 \mathrm{~cm}^{-1}$. HRMS (ESI): $m / z$ calculated for $\mathrm{C}_{28} \mathrm{H}_{31} \mathrm{ClNaO}_{2} \mathrm{Si}[\mathrm{M}+\mathrm{Na}]^{+}=485.1674$, found: 485.1671 .

5-((2-(4-fluorophenyl)-1-phenyl-1H-benzo[b] silol-1-yl)oxy)-2,5-dimethylhexan-2-ol (3c)

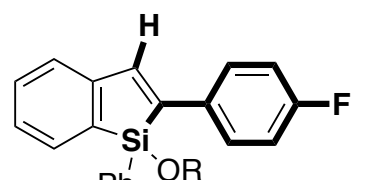

Following the General Procedure, the titled compound was obtained as colorless oil in 86\% yield (115.4 mg). ${ }^{1} \mathbf{H}$ NMR (400 MHz, $\left.\mathbf{C D C l}_{3}\right): \delta 1.11(\mathrm{~s}, 3 \mathrm{H}), 1.12(\mathrm{~s}, 3 \mathrm{H}), 1.18$ (s, $3 \mathrm{H}), 1.22(\mathrm{~s}, 3 \mathrm{H}), 1.38(\mathrm{brs}, 1 \mathrm{H}), 1.51-1.62(\mathrm{~m}, 4 \mathrm{H}), 6.96(\mathrm{dd}, J=8.7 \mathrm{~Hz}, 2 \mathrm{H}), 7.18(\mathrm{dd}, J$ $\mathrm{Ph}{ }^{\prime} \mathrm{OR}$ $=7.3 \mathrm{~Hz}, 1 \mathrm{H}), 7.26(\mathrm{~d}, J=7.3 \mathrm{~Hz}, 1 \mathrm{H}), 7.28-7.38(\mathrm{~m}, 4 \mathrm{H}), 7.47-7.52(\mathrm{~m}, 3 \mathrm{H}), 7.56(\mathrm{~s}, 1 \mathrm{H})$, 7.61-7.64 (m, 2H). ${ }^{13} \mathbf{C}$ NMR (100 MHz, $\left.\mathbf{C D C l}_{3}\right): \delta 29.2,29.27,29.29,29.6,38.4,39.0,70.8,76.5,115.6(\mathrm{~d}, J=22.0$ $\mathrm{Hz}), 124.8,127.7,128.2,128.5$ (d, $J=8.1 \mathrm{~Hz}), 130.3,131.0,132.8,133.8,134.3,134.5$ (q, $J=3.7 \mathrm{~Hz}), 135.3,141.7$ (d, $J=1.8 \mathrm{~Hz}), 142.0,147.8,162.2(\mathrm{~d}, J=247.2 \mathrm{~Hz}) .{ }^{19} \mathbf{F}$ NMR $\left(376 \mathbf{~ M H z}, \mathbf{C D C l}_{3}\right): \delta-114.7-114.6(\mathrm{~m})$. IR (ATR-IR): 3458, 2971, 1738, 1588, 1544, 1504, 1440, 1429, 1367, 1276, 1260, 1230, 1158, 1114, 1065, 1037, 996, 903, 831, 803, 756, 718, 698, 668, 598, 5600, 506, 490, 470, 429, 416, $402 \mathrm{~cm}^{-1}$. HRMS (ESI): $\mathrm{m} / z$ calculated for $\mathrm{C}_{28} \mathrm{H}_{31} \mathrm{FNaO}_{2} \mathrm{Si}$ $[\mathrm{M}+\mathrm{Na}]^{+}=469.1970$, found: 469.1967 .

\section{2,5-dimethyl-5-((1-phenyl-2-(4-(trifluoromethyl)phenyl)-1 H-benzo[b]silol-1-yl)oxy)hexan-2-ol (3d)}

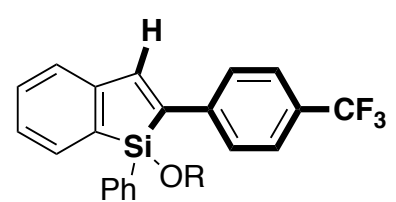

Following the General Procedure, the titled compound was obtained as colorless oil in 85\% yield (126.2 mg). ${ }^{1} \mathbf{H}$ NMR (500 MHz, $\left.\mathbf{C D C l}_{3}\right): \delta 1.06(\mathrm{brs}, 1 \mathrm{H}), 1.11(\mathrm{~s}, 3 \mathrm{H}), 1.12$ $(\mathrm{s}, 3 \mathrm{H}), 1.18(\mathrm{~s}, 3 \mathrm{H}), 1.23(\mathrm{~s}, 3 \mathrm{H}), 1.49-1.62(\mathrm{~m}, 4 \mathrm{H}), 7.24(\mathrm{dt}, J=7.3,1.2 \mathrm{~Hz}, 1 \mathrm{H})$, 7.30-7.33 (m, $3 \mathrm{H}), 7.38(\mathrm{dt}, J=7.6,1.2 \mathrm{~Hz}, 2 \mathrm{H}), 7.52(\mathrm{~d}, J=8.1 \mathrm{~Hz}, 2 \mathrm{H}), 7.54(\mathrm{~d}, J=8.7$ $\mathrm{Hz}, 1 \mathrm{H}), 7.59-7.63$ (m, 4H), $7.72(\mathrm{~s}, 1 \mathrm{H}) .{ }^{13} \mathbf{C}$ NMR (100 MHz, $\mathbf{C D C l}_{3}$ ): $\delta$ 29.2, 29.27, 29.31, 29.6, 38.4, 39.0, 70.8, $76.7,124.5$ (q, $J=271.6 \mathrm{~Hz}), 125.3,125.7(\mathrm{q}, J=3.7 \mathrm{~Hz}), 127.1,128.26,128.31,128.9(\mathrm{q}, J=32.3 \mathrm{~Hz}), 130.5,131.2$, 133.0, 133.4, 134.3, 135.6, 141.9, 142.0, 144.3, 147.4. ${ }^{19} \mathbf{F}$ NMR (470 $\mathbf{~ M H z , ~} \mathbf{C D C l}_{3}$ ): $\delta$-62.4. IR (ATR-IR): 3427, 2972, 1612, 1547, 1466, 1441, 1430, 1368, 1322, 1277, 1164, 1109, 1066, 1037, 1015, 997, 945, 906, 838, 758, 718, 698, 660, 601, 556, 493, 470, 456, 438, 428, $418 \mathrm{~cm}^{-1}$. HRMS (ESI): $\mathrm{m} / z$ calculated for $\mathrm{C}_{29} \mathrm{H}_{31} \mathrm{~F}_{3} \mathrm{NaO}_{2} \mathrm{Si}[\mathrm{M}+\mathrm{Na}]^{+}=$ 519.1938, found: 519.1938 .

\section{5-((2-(4-butylphenyl)-1-phenyl-1H-benzo[b]silol-1-yl)oxy)-2,5-dimethylhexan-2-ol (3e)}

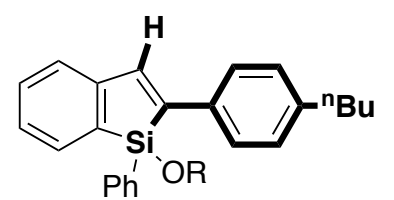

Following the General Procedure (reaction at $60{ }^{\circ} \mathrm{C}, \mathrm{GPC}: \mathrm{CHCl}_{3}$ ), the titled compound was obtained as colorless oil in 78\% yield $(113.9 \mathrm{mg}) .{ }^{1} \mathbf{H}$ NMR $(\mathbf{5 0 0} \mathbf{~ M H z}$, $\left.\mathbf{C D C l}_{3}\right): \delta 0.90(\mathrm{t}, J=7.3 \mathrm{~Hz}, 3 \mathrm{H}), 1.09(\mathrm{~s}, 3 \mathrm{H}), 1.10(\mathrm{~s}, 3 \mathrm{H}), 1.18(\mathrm{~s}, 3 \mathrm{H}) 1.24(\mathrm{~s}, 3 \mathrm{H})$ $1.30-1.37(\mathrm{~m}, 2 \mathrm{H}), 1.50-1.59(\mathrm{~m}, 6 \mathrm{H}), 2.56(\mathrm{t}, J=7.9 \mathrm{~Hz}, 2 \mathrm{H}), 7.09(\mathrm{~d}, J=7.9 \mathrm{~Hz}, 2 \mathrm{H})$, $7.16(\mathrm{dd}, J=7.0 \mathrm{~Hz}, 1 \mathrm{H}), 7.25(\mathrm{~d}, J=7.3 \mathrm{~Hz}, 1 \mathrm{H}), 7.28-7.37(\mathrm{~m}, 4 \mathrm{H}), 7.45(\mathrm{~d}, J=7.9 \mathrm{~Hz}, 2 \mathrm{H}), 7.50(\mathrm{~d}, J=7.0 \mathrm{~Hz}$, $1 \mathrm{H}), 7.61(\mathrm{~s}, 1 \mathrm{H}), 7.64(\mathrm{dd}, J=6.7,1.2 \mathrm{~Hz}, 2 \mathrm{H})$. The proton of hydroxy group was not detected. ${ }^{13} \mathbf{C}$ NMR $(\mathbf{1 2 5} \mathbf{~ M H z}$, $\mathbf{C D C l}_{3}$ ): $\delta 14.1,22.5,29.2$ (2C, overlapped), 29.3, 29.7, 33.6, 35.5, 38.4, 39.0, 70.8, 76.5, 124.6, 127.1, 127.5, 128.1, 
128.8, 130.1, 130.9, 132.8, 134.2, 134.4, 135.6, 135.7, 141.2, 142.3, 143.1, 148.1. IR (ATR-IR): 3403, 2966, 2929, $1588,1505,1439,1367,1219,1104,1064,1037,902,828,756,740,716,698,669,600,552 \mathrm{~cm}^{-1}$. HRMS (ESI): $m / z$ calculated for $\mathrm{C}_{32} \mathrm{H}_{40} \mathrm{NaO}_{2} \mathrm{Si}[\mathrm{M}+\mathrm{Na}]^{+}=507.2690$, found: 507.2691.

5-((2-(4-methoxyphenyl)-1-phenyl-1H-benzo[b] silol-1-yl)oxy)-2,5-dimethylhexan-2-ol (3f)

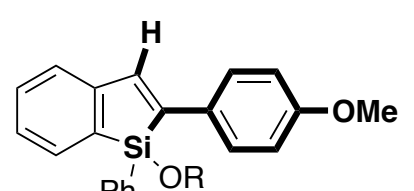

$\mathrm{Ph}$ 'OR

Following the General Procedure (reaction at $60{ }^{\circ} \mathrm{C}$ ), the titled compound was obtained as colorless oil in $40 \%$ yield $(55.3 \mathrm{mg}) .{ }^{1} \mathbf{H}$ NMR $\left(\mathbf{5 0 0} \mathbf{~ M H z}, \mathbf{C D C l}_{3}\right): \delta 1.11(\mathrm{~s}$, $3 \mathrm{H}), 1.12(\mathrm{~s}, 3 \mathrm{H}), 1.19(\mathrm{~s}, 3 \mathrm{H}), 1.23(\mathrm{~s}, 3 \mathrm{H}), 1.26(\mathrm{brs}, 1 \mathrm{H}), 1.52-1.60(\mathrm{~m}, 4 \mathrm{H}), 3.77(\mathrm{~s}$, $3 \mathrm{H}), 6.82(\mathrm{dt}, J=8.9,3.1 \mathrm{~Hz}, 2 \mathrm{H}), 7.15(\mathrm{dt}, J=7.5,0.9 \mathrm{~Hz}, 1 \mathrm{H}), 7.24(\mathrm{~d}, J=6.7 \mathrm{~Hz}$, $1 \mathrm{H}), 7.28-7.37(\mathrm{~m}, 4 \mathrm{H}), 7,47-7.50(\mathrm{~m}, 3 \mathrm{H}), 7.54(\mathrm{~s}, 1 \mathrm{H}), 7.63$ (dd, J = 7.9, $1.2 \mathrm{~Hz}, 2 \mathrm{H}) .{ }^{13} \mathbf{C}$ NMR (125 MHz, $\left.\mathbf{C D C l}_{3}\right): \delta 29.2,29.28,29.33,29.6,38.4,39.1,55.4,70.9,76.5,114.2,124.4,127.3,128.1,128.4,130.2,130.96,131.03$, 132.8, 134.2, 134.4, 135.3, 139.9, 142.5, 148.3, 159.1. IR (ATR-IR): 3412, 2968, 1604, 1587, 1506, 1464, 1440, 1429, 1384, 1367, 1298, 1358, 1176, 1105, 1064, 1033, 946, 901, 828, 786, 669, 625, 598, 564, $516 \mathrm{~cm}^{-1}$. HRMS (ESI): $\mathrm{m} / z$ calculated for $\mathrm{C}_{29} \mathrm{H}_{34} \mathrm{NaO}_{3} \mathrm{Si}[\mathrm{M}+\mathrm{Na}]^{+}=481.2169$, found: 481.2169 .

5-((2-(3,5-bis(trifluoromethyl)phenyl)-1-phenyl-1H-benzo[b] silol-1-yl)oxy)-2,5-dimethylhexan-2-ol (3g)

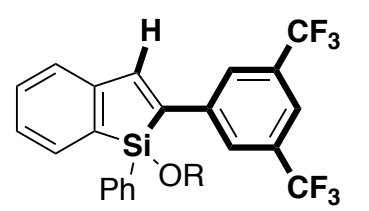

Following the General Procedure, the titled compound was obtained as yellow oil in $76 \%$ yield (128.0 mg). ${ }^{1} \mathbf{H}$ NMR (400 MHz, $\left.\mathbf{C D C l}_{3}\right): \delta 1.13(\mathrm{~s}, 3 \mathrm{H}), 1.15(\mathrm{~s}, 3 \mathrm{H}), 1.20(\mathrm{~s}, 3 \mathrm{H})$, $1.25(\mathrm{~s}, 3 \mathrm{H}), 1.52-1.67(\mathrm{~m}, 4 \mathrm{H}), 7.28-7.46(\mathrm{~m}, 6 \mathrm{H}), 7.58-7.60(\mathrm{~m}, 3 \mathrm{H}), 7.67(\mathrm{~s}, 1 \mathrm{H}), 7.74(\mathrm{~s}$,

$1 \mathrm{H}), 7.96$ (s, 2H). The proton of hydroxy group was not observed. ${ }^{13} \mathbf{C}$ NMR (125 $\mathbf{~ M H z}$, $\mathbf{C D C l}_{3}$ ): $\delta 29.2,29.26,29.34,29.7,38.4,39.1,70.8,77.0,120.5$ (apparent t $J=3.6 \mathrm{~Hz}$ ), $123.6(\mathrm{q}, J=272.5 \mathrm{~Hz}$ ), 125.7, 126.6, 128.4, 128.8, 130.7, 131.5, $132.0(\mathrm{q}, J=32.7 \mathrm{~Hz}), 132.8,133.3,134.3,135.0,140.6,140.8,144.8,147.2 .{ }^{19} \mathbf{F}$ NMR (470 MHz, CDCl $_{3}$ ): $\delta$-63.0. IR (ATR-IR): 3487, 2967, 1542, 1466, 1440, 1429, 1375, 1315, 1278, 1226, 1173 , $1122,1065,1037,995,890,857,844,760,741,718,699,682,660,590,562,541,508 \mathrm{~cm}^{-1}$. HRMS (ESI): $\mathrm{m} / z$ calculated for $\mathrm{C}_{30} \mathrm{H}_{30} \mathrm{~F}_{6} \mathrm{NaO}_{2} \mathrm{Si}[\mathrm{M}+\mathrm{Na}]^{+}=587.1812$, found: 587.1810 .

\section{5-((2-mesityl-1-phenyl-1 H-benzo[b]silol-1-yl)oxy)-2,5-dimethylhexan-2-ol (3h)}

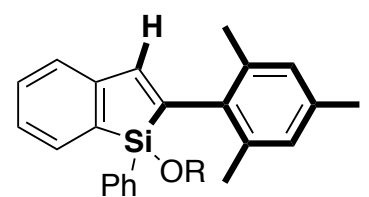

Following the General Procedure (reaction at $60^{\circ} \mathrm{C}$ ), the titled compound was obtained as colorless oil in $71 \%$ yield $(100.0 \mathrm{mg}) .{ }^{1} \mathbf{H}$ NMR $\left(\mathbf{5 0 0} \mathbf{~ M H z}, \mathbf{C D C l}_{3}\right): \delta 0.95$ (brs, $\left.1 \mathrm{H}\right)$, $1.046(\mathrm{~s}, 3 \mathrm{H}), 1.052(\mathrm{~s}, 3 \mathrm{H}), 1.256(\mathrm{~s}, 3 \mathrm{H}), 1.260(\mathrm{~s}, 3 \mathrm{H}), 1.43-1.58(\mathrm{~m}, 4 \mathrm{H}), 1.94(\mathrm{~s}, 6 \mathrm{H})$, $2.26(\mathrm{~s}, 3 \mathrm{H}), 6.80(\mathrm{~s}, 2 \mathrm{H}), 6.88(\mathrm{~s}, 1 \mathrm{H}), 7.21(\mathrm{~d}, J=7.3 \mathrm{~Hz}, 1 \mathrm{H}), 7.24(\mathrm{dd}, J=7.6 \mathrm{~Hz}, 2 \mathrm{H})$, $7.30(\mathrm{ddd}, J=7.3,7.3,0.6 \mathrm{~Hz}, 1 \mathrm{H}), 7.35(\mathrm{tt}, J=7.3,1.2 \mathrm{~Hz}, 1 \mathrm{H}), 7.40(\mathrm{ddd}, J=7.5,7.5,1.2 \mathrm{~Hz}, 1 \mathrm{H}), 7.49$ (dd, $J=7.3$, $1.2 \mathrm{~Hz}, 2 \mathrm{H}), 7.74(\mathrm{dd}, J=7.0,0.6 \mathrm{~Hz}, 1 \mathrm{H}) .{ }^{13} \mathbf{C}$ NMR (125 MHz, $\left.\mathbf{C D C l}_{3}\right): \delta 20.9,21.1,29.1,29.3,30.27,30.28,38.3$, 38.9, 70.7, 76.5, 124.5, 127.3, 127.9, 128.0, 130.2, 131.0, 133.2, 133.3, 134.7, 135.0, 135.4, 135.9, 137.2, 145.5, 146.4, 149.0. IR (ATR-IR): 3403, 2969, 1577, 1439, 1366, 1279, 1221, 1104, 1063, 1036, 904, 850, 756, 741, 700, 662, 622, $607,565,520 \mathrm{~cm}^{-1}$. HRMS (ESI): $\mathrm{m} / z$ calculated for $\mathrm{C}_{31} \mathrm{H}_{38} \mathrm{NaO}_{2} \mathrm{Si}[\mathrm{M}+\mathrm{Na}]^{+}=493.2533$, found: 493.2534 .

\section{2,5-dimethyl-5-((2-(naphthalen-1-yl)-1-phenyl-1H-benzo[b]silol-1-yl)oxy)hexan-2-ol (3i)}

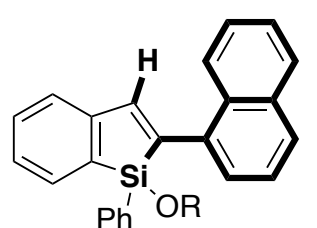

Following the General Procedure, the titled compound was obtained as colorless oil in $90 \%$ yield (129.7 mg). ${ }^{1} \mathbf{H}$ NMR (400 MHz, $\mathbf{C D C l}_{3}$ ): $\delta 0.82$ (brs, 1H), 0.94 (s, 3H), 0.97 (s, 3H), $1.18(\mathrm{~s}, 3 \mathrm{H}), 1.24(\mathrm{~s}, 3 \mathrm{H}), 1.34-1.52(\mathrm{~m}, 4 \mathrm{H}), 7.22(\mathrm{dd}, J=7.1,1.2 \mathrm{~Hz}, 1 \mathrm{H}), 7.27-7.32(\mathrm{~m}, 3 \mathrm{H})$, $7.34(\mathrm{~d}, J=7.6 \mathrm{~Hz}, 2 \mathrm{H}), 7.38(\mathrm{dt}, J=8.3,1.5 \mathrm{~Hz}, 1 \mathrm{H}), 7.39-7.46(\mathrm{~m}, 3 \mathrm{H}), 7.51(\mathrm{~s}, 1 \mathrm{H}), 7.60(\mathrm{~d}$, $J=6.9 \mathrm{~Hz}, 1 \mathrm{H}), 7.64(\mathrm{dd}, J=8.0,1.5 \mathrm{~Hz}, 2 \mathrm{H}), 7.67(\mathrm{~d}, J=8.1 \mathrm{~Hz}, 1 \mathrm{H}), 7.83(\mathrm{~d}, J=8.1 \mathrm{~Hz}, 1 \mathrm{H})$, $8.25(\mathrm{~d}, J=8.3 \mathrm{~Hz}, 1 \mathrm{H}) .{ }^{13} \mathbf{C}$ NMR (125 $\left.\mathbf{~ M H z}, \mathbf{C D C l}_{3}\right): \delta 29.0,29.1,29.3,29.9,38.3,38.8,70.6,76.3,124.8,125.1$, S-13 
125.5, 125.7 (2C, overlapped), 126.4, 126.8, 127.9, 128.2 (2C, overlapped), 128.5, 130.3, 131.0, 131.5, 132.9, 134.3, 134.4, 134.6 (2C, overlapped), 135.0, 138.4, 143.3, 147.8, 148.0. IR (ATR-IR): 3433, 2970, 1589, 1439, 1367, 1217, 1112, 1066, 1037, 906, 799, 755, 716, 698, 670, 652, 608, 577, $504 \mathrm{~cm}^{-1}$. HRMS (ESI): $\mathrm{m} / z$ calculated for $\mathrm{C}_{32} \mathrm{H}_{34} \mathrm{NaO}_{2} \mathrm{Si}[\mathrm{M}+\mathrm{Na}]^{+}=501.2220$, found: 501.2219 .

\section{2,5-dimethyl-5-((1-phenyl-2-(thiophen-2-yl)-1H-benzo[b] silol-1-yl)oxy)hexan-2-ol (3j)}

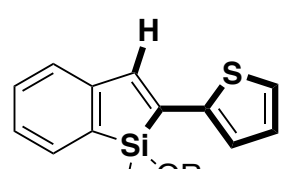

$\mathrm{Ph}$ 'OR

Following the General Procedure, the titled compound was obtained as colorless oil in 58\% yield (75.0 mg). ${ }^{1} \mathbf{H}$ NMR (400 MHz, $\left.\mathbf{C D C l}_{3}\right): \delta 1.09$ (brs, $\left.1 \mathrm{H}\right), 1.13$ (s, 3H), 1.14 (s, 3H), 1.22 $(\mathrm{s}, 3 \mathrm{H}), 1.25(\mathrm{~s}, 3 \mathrm{H}), 1.53-1.61(\mathrm{~m}, 4 \mathrm{H}), 6.92(\mathrm{dd}, J=5.1,3.4 \mathrm{~Hz}, 1 \mathrm{H}), 6.99(\mathrm{dd}, J=3.4,0.7 \mathrm{~Hz}$, $1 \mathrm{H}), 7.16-7.20(\mathrm{~m}, 2 \mathrm{H}), 7.24(\mathrm{~d}, J=7.6 \mathrm{~Hz}, 1 \mathrm{H}), 7.30-7.41(\mathrm{~m}, 4 \mathrm{H}), 7.44(\mathrm{~s}, 1 \mathrm{H}), 7.51(\mathrm{dd}, J=$ $7.1 \mathrm{~Hz}, 1 \mathrm{H}), 7.64$ (dd, $J=8.1,1.5 \mathrm{~Hz}, 2 \mathrm{H}) .{ }^{13} \mathbf{C} \mathbf{~ N M R}\left(\mathbf{1 2 5} \mathbf{~ M H z}, \mathbf{C D C l}_{3}\right): \delta 29.2,29.28,29.34,29.6,38.4,39.1,70.8$, 76.7, 124.5, 124,6, 127.0, 127.5, 127.7, 128.1, 130.4, 131.1, 132.9, 133.4, 134.5, 134.8, 136.9, 140.6, 143.3, 148.0. IR (ATR-IR): 3464, 2962, 1731, 1586, 1498, 1444, 1427, 1364, 1336, 1306, 1254, 1231, 1114, 1063, 1022, 941, 891, 845, $818,742,717,698,670,599,558,526 \mathrm{~cm}^{-1}$. HRMS (ESI): $\mathrm{m} / z$ calculated for $\mathrm{C}_{26} \mathrm{H}_{30} \mathrm{NaO}_{2} \mathrm{SSi}[\mathrm{M}+\mathrm{Na}]^{+}=457.1628$, found: 457.1629 .

(E)-2,5-dimethyl-5-((1-phenyl-2-styryl-1H-benzo[b]silol-1-yl)oxy)hexan-2-ol (3k)

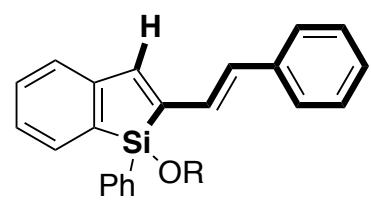

Following the General Procedure, the titled compound was obtained as yellow viscose oil in 97\% yield (132.6 mg). ${ }^{1} \mathbf{H}$ NMR (400 MHz, $\left.\mathbf{C D C l}_{3}\right): \delta 1.16(\mathrm{~s}, 3 \mathrm{H}), 1.17(\mathrm{~s}, 3 \mathrm{H})$, $1.269(\mathrm{~s}, 3 \mathrm{H}), 1.272(\mathrm{~s}, 3 \mathrm{H}), 1.61-1.64(\mathrm{~m}, 4 \mathrm{H}), 6.72(\mathrm{~d}, J=15.9 \mathrm{~Hz}, 1 \mathrm{H}), 7.16-7.22(\mathrm{~m}$, $4 \mathrm{H}), 7.26-7.40(\mathrm{~m}, 9 \mathrm{H}), 7.51(\mathrm{~d}, J=6.9 \mathrm{~Hz}, 1 \mathrm{H}), 7.65(\mathrm{dd}, J=8.0,1.5 \mathrm{~Hz}, 2 \mathrm{H})$. The proton of hydroxy group was not observed. ${ }^{13} \mathbf{C}$ NMR (125 $\left.\mathbf{~ M H z}, \mathbf{C D C l}_{3}\right): \delta 29.26,29.32,29.4,29.7,38.5,39.2,70.9$, 76.5, 124.7, 126.5, 127.6, 127.7, 128.1, 128.7, 129.2, 130.2, 131.0, 132.9, 134.0, 134.2, 134.4, 135.7, 137.8, 143.4, 145.8, 148.2. IR (ATR-IR): 3389, 3053, 2970, 2932, 2871, 1586, 1438, 1430, 1384, 1367, 1273, 1250, 1228, 1113, 1105, 1062, 1021, 997, 959, 907, 843, 748, 716, 698, 662, 589, 553, 544, 528, 503, 478, 468, 450, 438, 422, 414, $404 \mathrm{~cm}^{-1}$. HRMS (ESI): $m / z$ calculated for $\mathrm{C}_{30} \mathrm{H}_{34} \mathrm{NaO}_{2} \mathrm{Si}[\mathrm{M}+\mathrm{Ag}]^{+}=561.1374$, found: 561.1371 .

\section{2,5-dimethyl-5-((1-phenyl-2-(phenylethynyl)-1H-benzo[b]silol-1-yl)oxy)hexan-2-ol (31)}

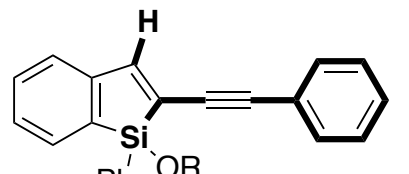

Following the General Procedure, the titled compound was obtained as brown oil in 59\% yield (80.2 mg). ${ }^{1} \mathbf{H}$ NMR (500 MHz, $\left.\mathbf{C D C l}_{3}\right): 1.13$ (s, 3H), 1.14 (s, 3H), 1.31 (s, $3 \mathrm{H}), 1.35(\mathrm{~s}, 3 \mathrm{H}), 1.58-1.66(\mathrm{~m}, 4 \mathrm{H}), 7.21-7.30(\mathrm{~m}, 5 \mathrm{H}), 7.32-7.36(\mathrm{~m}, 3 \mathrm{H}), 7.39(\mathrm{dt}, J$ $=7.3,1.5 \mathrm{~Hz}, 1 \mathrm{H}), 7.42(\mathrm{dd}, J=7.9,1.2 \mathrm{~Hz}, 2 \mathrm{H}), 7.55(\mathrm{dd}, J=6.7,0.6 \mathrm{~Hz}, 1 \mathrm{H}), 7.56(\mathrm{~s}$, $1 \mathrm{H}), 7.70(\mathrm{dd}, J=8.1,1.2 \mathrm{~Hz}, 2 \mathrm{H})$. The proton of hydroxy group was not detected. ${ }^{13} \mathbf{C}$ NMR $\left(\mathbf{1 2 5} \mathbf{~ M H z}, \mathbf{C D C l}_{3}\right): \delta$ 29.26, 29.29, 29.5, 29.8, 38.4, 39.0, 70.8, 76.8, 89.3, 97.8, 124.3, 125.1, 125.7, 128.0, 128.1, 128.4, 128.6, 130.5, 131.1, 131.6, 132.8, 132.9, 134.0, 134.5, 147.0, 152.6. IR (ATR-IR): 3420, 2968, 1589, 1487, 1440, 1367, 1221, 1115, 1063, 1037, 1023, 904, 859, 754, 716, 690, 666, 600, 554, $520 \mathrm{~cm}^{-1}$. HRMS (ESI): $\mathrm{m} / z$ calculated for $\mathrm{C}_{30} \mathrm{H}_{32} \mathrm{NaO}_{2} \mathrm{Si}$ $[\mathrm{M}+\mathrm{Na}]^{+}=475.2064$, found: 475.2064 . 
5-((2-((4-(tert-butyl)phenyl)thio)-1-phenyl-1H-benzo[b]silol-1-yl)oxy)-2,5-dimethylhexan-2-ol (3m)

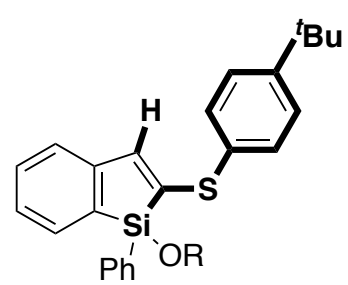

Following the General Procedure, the titled compound was obtained as colorless oil in 41\% yield (64.0 mg). ${ }^{1} \mathbf{H}$ NMR (500 MHz, $\mathbf{C D C l}_{3}$ ): $\delta 1.19$ (s, 6H), 1.30 (s, 3H), 1.32 (s, 9H), $1.34(\mathrm{~s}, 3 \mathrm{H}), 1.59-1.70(\mathrm{~m}, 4 \mathrm{H}), 6.59(\mathrm{~s}, 1 \mathrm{H}), 6.94(\mathrm{~d}, J=7.6 \mathrm{~Hz}, 1 \mathrm{H}), 7.06(\mathrm{dd}, J=$ 7.3, 7.0 Hz, 1H), 7.21-7.25 (m, 1H), 730-7.33 (m, 4H), 7.38 (dt, J= 7.6, $1.2 \mathrm{~Hz}, 1 \mathrm{H}), 7.40$ $(\mathrm{d}, J=8.2 \mathrm{~Hz}, 2 \mathrm{H}), 7.44(\mathrm{dd}, J=7.0,0.6 \mathrm{~Hz}, 1 \mathrm{H}), 7.58(\mathrm{dd}, J=7.0,0.6 \mathrm{~Hz}, 2 \mathrm{H})$. The proton of hydroxy group was not detected. ${ }^{13} \mathbf{C}$ NMR $\left(125 \mathbf{~ M H z}, \mathbf{C D C l}_{3}\right): \delta 29.4$ (2C, overlapped), 29.5, 29.6, 31.4 (3C, overlapped), 34.8, 38.5, 39.0, 71.0, 76.8, 122.8, 126.2, 126.3, 128.0, 128.5, 130.3, 131.0, 132.8, 133.1, 134.2, 134.3, 134.5, 136.8, 142.6, 148.1, 151.8. IR (ATR-IR): 3382, 2965, 1587, 1518, 1488, 1429, 1384, 1366, 1266, 1117, 1066, 1037, 907, 828, 754, 716, 697, 667, 599, 554, 534, $514 \mathrm{~cm}^{-1}$. HRMS (ESI): $\mathrm{m} / z$ calculated for $\mathrm{C}_{32} \mathrm{H}_{40} \mathrm{NaO}_{2} \mathrm{SSi}[\mathrm{M}+\mathrm{Na}]^{+}=539.2411$, found: 539.2410 .

\section{5-((2-(diphenylphosphaneyl)-1-phenyl-1H-benzo[b]silol-1-yl)oxy)-2,5-dimethylhexan-2-ol (3n)}

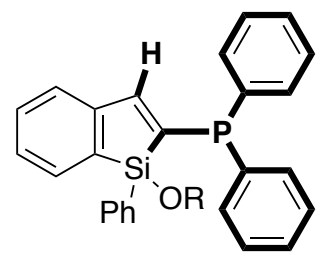

Following the General Procedure (reaction at $70^{\circ} \mathrm{C}$ ), the titled compound was obtained as yellow oil in 94\% yield (150.6 mg). ${ }^{1} \mathbf{H}$ NMR (400 $\left.\mathbf{~ M H z}, \mathbf{C D C l}_{3}\right): \delta 1.151(\mathrm{~s}, 3 \mathrm{H}), 1.153(\mathrm{~s}$, $3 \mathrm{H}), 1.209(\mathrm{~s}, 3 \mathrm{H}), 1.213(\mathrm{~s}, 3 \mathrm{H}), 1.47-1.56(\mathrm{~m}, 4 \mathrm{H}), 7.01\left(\mathrm{~d}, J_{\mathrm{H}-\mathrm{P}}=10.7 \mathrm{~Hz}, 1 \mathrm{H}\right), 7.02-7.21$ $(\mathrm{m}, 6 \mathrm{H}), 7.22(\mathrm{~d}, J=7.1 \mathrm{~Hz}, 1 \mathrm{H}), 7.25-7.35(\mathrm{~m}, 9 \mathrm{H}), 7.39-7.44(\mathrm{~m}, 2 \mathrm{H}), 7.53(\mathrm{~d}, J=6.9 \mathrm{~Hz}$, $1 \mathrm{H})$. The proton of hydroxy group was not detected. ${ }^{13} \mathbf{C} \mathbf{~ N M R}\left(\mathbf{1 0 0} \mathbf{~ M H z}, \mathbf{C D C l}_{3}\right): \delta 29.3$, 29.4, 29.6, 29.7, 38.4, 38.9, 70.9, 76.4, 124.4, 127.66, $127.72\left(\mathrm{~d}, J_{\mathrm{C}-\mathrm{P}}=9.5 \mathrm{~Hz}\right), 128.1\left(\mathrm{~d}, J_{\mathrm{C}-\mathrm{P}}=7.3 \mathrm{~Hz}\right), 128.4\left(\mathrm{~d}, J_{\mathrm{C}-\mathrm{P}}=\right.$ $7.3 \mathrm{~Hz}$ ), 128.8 (d, $\left.J_{\mathrm{C}-\mathrm{P}}=2.9 \mathrm{~Hz}\right), 129.9,130.9,133.0,134.1,134.3,134.4,134.6,134.8,135.5$ (d, $\left.J_{\mathrm{C}-\mathrm{P}}=8.8 \mathrm{~Hz}\right), 136.3$ $\left(\mathrm{d}, J_{\mathrm{C}-\mathrm{P}}=2.9 \mathrm{~Hz}\right), 136.7\left(\mathrm{~d}, J_{\mathrm{C}-\mathrm{P}}=12.5 \mathrm{~Hz}\right), 142.2\left(\mathrm{~d}, J_{\mathrm{C}-\mathrm{P}}=30.8 \mathrm{~Hz}\right), 148.2\left(\mathrm{~d}, J_{\mathrm{C}-\mathrm{P}}=7.3 \mathrm{~Hz}\right), 152.0\left(\mathrm{~d}, J_{\mathrm{C}-\mathrm{P}}=8.8 \mathrm{~Hz}\right)$. IR (ATR-IR): 3387, 3052, 2968, 1586, 1519, 1434, 1366, 1103, 1065, 1038, 998, 948, 905, 829, 740, 694, 666, 525 $\mathrm{cm}^{-1}$. HRMS (ESI): $m / z$ calculated for $\mathrm{C}_{34} \mathrm{H}_{37} \mathrm{NaO}_{2} \mathrm{PSi}[\mathrm{M}+\mathrm{Na}]^{+}=559.2193$, found: 559.2193 .

\section{5-((2-(9H-carbazol-9-yl)-1-phenyl-1H-benzo[b] silol-1-yl)oxy)-2,5-dimethylhexan-2-ol (3o)}

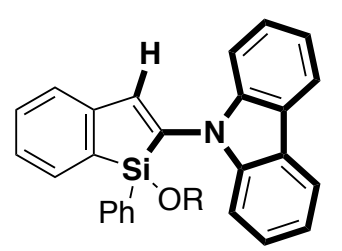

Following the General Procedure $(0.2 \mathrm{mmol} \mathrm{scale})$, the titled compound was obtained as yellow oil in 91\% yield (94.2 mg). ${ }^{1} \mathbf{H}$ NMR (500 $\left.\mathbf{~ M H z}, \mathbf{C D C l}_{3}\right): \delta 0.90(\mathrm{~s}, 3 \mathrm{H}), 0.93(\mathrm{~s}, 3 \mathrm{H})$, $1.13(\mathrm{~s}, 3 \mathrm{H}), 1.18-1.26(\mathrm{~m}, 6 \mathrm{H}), 1.37-1.49(\mathrm{~m}, 2 \mathrm{H}), 7.19(\mathrm{dd}, J=7.3,7.3 \mathrm{~Hz}, 2 \mathrm{H}), 7.24-7.28$ $(\mathrm{m}, 4 \mathrm{H}), 7.31(\mathrm{dd}, J=7.6,7.6 \mathrm{~Hz}, 2 \mathrm{H}), 7.38-7.42(\mathrm{~m}, 2 \mathrm{H}), 7.55(\mathrm{~d}, J=7.0 \mathrm{~Hz}, 1 \mathrm{H}), 7.58(\mathrm{~d}, J$ $=8.2 \mathrm{~Hz}, 2 \mathrm{H}), 7.60(\mathrm{dd}, J=8.2,1.2 \mathrm{~Hz}, 2 \mathrm{H}), 7.61(\mathrm{~s}, 1 \mathrm{H}), 8.02(\mathrm{~d}, J=7.6 \mathrm{~Hz}, 2 \mathrm{H}) .{ }^{13} \mathbf{C}$ NMR

(125 MHz, $\mathbf{C D C l}_{3}$ ): $\delta 28.9,29.0$ (2C, overlapped), 29.9, 38.1, 38.7, 70.5, 77.0, 112.1, 120.0, 120.1, 124.1, 124.4, 125.8, 127.7, 128.4, 130.7, 131.4, 132.4, 133.1, 134.0, 134.7, 139.4, 140.8, 142.6, 146.1. IR (ATR-IR): 3421, 2969, 1590, 1539, 1477, 1448, 1439, 1335, 1318, 1278, 1220, 1102, 1065, 1038, 905, 747, 722, 698, 666, 640, 588, 573, 534, 506 $\mathrm{cm}^{-1}$. HRMS (ESI): $m / z$ calculated for $\mathrm{C}_{34} \mathrm{H}_{35} \mathrm{NNaO}_{2} \mathrm{Si}[\mathrm{M}+\mathrm{Na}]^{+}=540.2329$, found: 540.2330 .

\section{5,5'-((1,4-phenylenebis(1-phenyl-1H-benzo[b]silole-2,1-diyl))bis(oxy))bis(2,5-dimethylhexan-2-ol) (3p)}

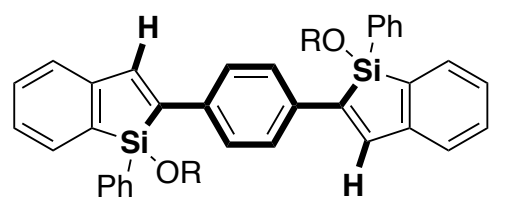

Following the General Procedure (reaction at $70^{\circ} \mathrm{C}$, 4.0 equiv of ${ }^{t} \mathrm{BuLi}$ and $\mathbf{1 a}$ were used), the titled compound was obtained as yellow oil in $83 \%$ yield (193.3 mg). ${ }^{1} \mathbf{H}$ NMR (500 MHz, $\left.\mathbf{C D C l}_{3}\right): \delta 1.05$ (d, $\left.J=6.4 \mathrm{~Hz}, 5 \mathrm{H}\right), 1.09(\mathrm{~s}, 7 \mathrm{H})$, 1.16-1.23 (m, 12H), 1.27 (brs, $2 \mathrm{H}), 1.49-1.61(\mathrm{~m}, 8 \mathrm{H}), 7.16(\mathrm{t}, J=7.0 \mathrm{~Hz}, 2 \mathrm{H})$, 7.23-7.35 (m, 10H), $7.47(\mathrm{~s}, 4 \mathrm{H}), 7.49(\mathrm{~d}, J=7.0 \mathrm{~Hz}, 2 \mathrm{H}), 7.62-7.63(\mathrm{~m}, 6 \mathrm{H}) .{ }^{13} \mathbf{C}$ NMR (125 MHz, CDCl $): \delta 29.19$ (2C, overlapped), 29.22 (2C, overlapped), 29.26, 29.29, 29.6, 29.7, 38.39, 38.42, 39.00, 39.04, 70.71, 70.74, 76.5 (2C, overlapped), 124.7, 127.4, 127.6, 128.1, 130.2, 130.9, 132.8, 134.0, 134.4, 135.6, 137.28, 137.32, 141.7, 142.65, 142.72, 
147.9. IR (ATR-IR): 3574, 2967, 1586, 1429, 1367, 1260, 1103, 1064, 1039, 901, 833, 758, 741, 716, 697, 668, 601, $571,512 \mathrm{~cm}^{-1}$. HRMS (ESI): $m / z$ calculated for $\mathrm{C}_{50} \mathrm{H}_{58} \mathrm{NaO}_{4} \mathrm{Si}_{2}[\mathrm{M}+\mathrm{Na}]^{+}=801.3766$, found: 801.3767.

\section{5-((1,2-diphenyl-1H-naphtho[2,3-b] silol-1-yl)oxy)-2,5-dimethylhexan-2-ol (3q)}

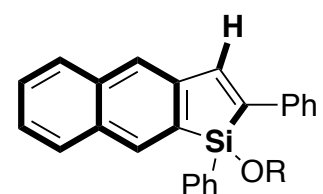

Following the General Procedure, the titled compound was obtained as colorless oil in 91\% yield (130.5 mg). ${ }^{1} \mathbf{H}$ NMR (400 MHz, $\left.\mathbf{C D C l}_{3}\right): \delta 1.05$ (brs, $\left.1 \mathrm{H}\right), 1.11$ (s, 3H), 1.12 (s, 3H), $1.18(\mathrm{~s}, 3 \mathrm{H}), 1.25(\mathrm{~s}, 3 \mathrm{H}), 1.52-1.64(\mathrm{~m}, 4 \mathrm{H}), 7.21(\mathrm{dt}, J=7.3,1.7 \mathrm{~Hz}, 1 \mathrm{H}), 7.28-7.33(\mathrm{~m}$, $4 \mathrm{H}), 7.36-7.43(\mathrm{~m}, 2 \mathrm{H}), 7.47(\mathrm{ddd}, J=7.6,7.3,1.5 \mathrm{~Hz}, 1 \mathrm{H}), 7.57(\mathrm{dd}, J=8.0,1.2 \mathrm{~Hz}, 2 \mathrm{H})$, 7.67-7.69 (m, 3H), $7.75(\mathrm{~d}, J=8.1 \mathrm{~Hz}, 1 \mathrm{H}), 7.80(\mathrm{~d}, J=8.1 \mathrm{~Hz}, 1 \mathrm{H}), 7.84(\mathrm{~s}, 1 \mathrm{H}), 7.97(\mathrm{~s}, 1 \mathrm{H}) .{ }^{13} \mathbf{C}$ NMR (125 MHz, $\left.\mathbf{C D C l}_{3}\right): \delta 29.21,29.22,29.3,29.7,38.4,39.1,70.8,76.5,123.0,125.9,127.15,127.22,127.5,128.1,128.3,128.65$, 128.74, 130.2, 133.6, 134.0, 134.3, 134.4, 134.5, 135.4, 138.5, 142.9, 144.4, 144.8. IR (ATR-IR): 3409, 2968, 1620, 1598, 1542, 1487, 1429, 1384, 1367, 1219, 1102, 1039, 902, 797, 747, 712, 695, 636, 589, 561, $517 \mathrm{~cm}^{-1}$. HRMS (ESI): $m / z$ calculated for $\mathrm{C}_{32} \mathrm{H}_{34} \mathrm{NaO}_{2} \mathrm{Si}[\mathrm{M}+\mathrm{Na}]^{+}=501.2220$, found: 501.2220 .

\section{5-((1,2-diphenyl-1H-naphtho[1,2-b] silol-1-yl)oxy)-2,5-dimethylhexan-2-ol (3r)}

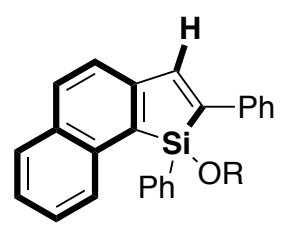

Following the General Procedure (reaction at $70^{\circ} \mathrm{C}$ ), the titled compound was obtained as light-green oil in 97\% yield (140 mg). ${ }^{1} \mathbf{H}$ NMR (500 $\left.\mathbf{~ M H z}, \mathbf{C D C l}_{3}\right): \delta 1.012(\mathrm{~s}, 3 \mathrm{H}), 1.014(\mathrm{~s}$, $3 \mathrm{H}), 1.14(\mathrm{~s}, 3 \mathrm{H}), 1.15(\mathrm{~s}, 3 \mathrm{H}), 1.46-1.56(\mathrm{~m}, 4 \mathrm{H}), 7.16-7.19(\mathrm{~m}, 1 \mathrm{H}), 7.26-7.36(\mathrm{~m}, 7 \mathrm{H}), 7.46$ $(\mathrm{d}, J=8.2 \mathrm{~Hz}, 1 \mathrm{H}), 7.55(\mathrm{~d}, J=7.3 \mathrm{~Hz}, 2 \mathrm{H}), 7.72(\mathrm{dd}, J=7.0,1.2 \mathrm{~Hz}, 2 \mathrm{H}), 7.74-7.76(\mathrm{~m}, 1 \mathrm{H})$, $7.76(\mathrm{~s}, 1 \mathrm{H}), 7.78(\mathrm{~d}, J=7.9 \mathrm{~Hz}, 1 \mathrm{H}), 7.86(\mathrm{~d}, J=8.2 \mathrm{~Hz}, 1 \mathrm{H})$. The proton of hydroxy group was not detected. ${ }^{13} \mathbf{C}$ NMR (125 MHz, $\mathbf{C D C l}_{3}$ ): $\delta 29.07$ (2C, overlapped), 29.10, 29.4, 38.5, 39.2, 70.7, 76.8, 123.8, 125.4, 126.9 , 127.2, 127.4, 128.3, 128.73, 128.74, 128.78, 130.2, 131.7, 133.0, 133.4, 133.9, 134.3, 135.8, 138.3, 142.0, 143.3, 146.9. IR (ATR-IR): 3403, 2970, 1583, 1507, 1466, 1429, 1367, 1217, 1136, 1103, 1037, 997, 977, 893, 818, 781, 743, 709, 693, 587, 563, 536, 526, $503 \mathrm{~cm}^{-1}$. HRMS (ESI): $\mathrm{m} / z$ calculated for $\mathrm{C}_{32} \mathrm{H}_{34} \mathrm{NaO}_{2} \mathrm{Si}[\mathrm{M}+\mathrm{Na}]^{+}=501.2220$, found: 501.2220 .

\section{5-((5,6-difluoro-1,2-diphenyl-1H-benzo[b] silol-1-yl)oxy)-2,5-dimethylhexan-2-ol (3s)}

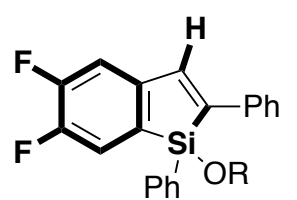

Following the General Procedure, the titled compound was obtained as colorless oil in 87\% yield (121.8 mg). ${ }^{1} \mathbf{H}$ NMR (500 MHz, $\left.\mathbf{C D C l}_{3}\right): \delta 0.86$ (brs, $\left.1 \mathrm{H}\right), 1.11(\mathrm{~s}, 3 \mathrm{H}), 1.12(\mathrm{~s}, 3 \mathrm{H})$, $1.17(\mathrm{~s}, 3 \mathrm{H}), 1.23(\mathrm{~s}, 3 \mathrm{H}), 1.50-1.61(\mathrm{~m}, 4 \mathrm{H}), 7.08(\mathrm{dd}, J=10.5,7.0 \mathrm{~Hz}, 1 \mathrm{H}), 7.20(\mathrm{t}, J=7.3 \mathrm{~Hz}$ $1 \mathrm{H}), 7.26-7.30(\mathrm{~m}, 3 \mathrm{H}), 7.32(\mathrm{~d}, J=7.3 \mathrm{~Hz}, 2 \mathrm{H}), 7.38(\mathrm{tt}, J=7.3,1.2 \mathrm{~Hz}, 1 \mathrm{H}), 7.49(\mathrm{dd}, J=7.9$, $1.2 \mathrm{~Hz}, 2 \mathrm{H}), 7.53(\mathrm{~s}, 1 \mathrm{H}), 7.61(\mathrm{dd}, J=7.9,1.2 \mathrm{~Hz}, 2 \mathrm{H}) .{ }^{13} \mathbf{C}$ NMR $\left(125 \mathbf{~ M H z}, \mathbf{C D C l}_{3}\right): \delta 29.25,29.26(2 \mathrm{C}$, overlapped), 29.6, 38.4 39.0, 70.7, 76.9, 113.9 (d, $J=17.3 \mathrm{~Hz}$ ), 121.6 (d, $J=16.4 \mathrm{~Hz}$ ), 127.1, 127.8, 128.3, 128.8, 130.6, 132.2 (dd, $J=3.6,3.6 \mathrm{~Hz}), 132.9,134.3,137.7,139.8,144.7$ (dd, $J=3.6,3.6 \mathrm{~Hz}), 149.3$ (d, $J=12.7 \mathrm{~Hz}), 151.3(\mathrm{dd}, J$ $=12.7,8.2 \mathrm{~Hz}), 153.3(\mathrm{~d}, J=13.6 \mathrm{~Hz}) .{ }^{19} \mathbf{F}$ NMR (470 MHz, $\left.\mathbf{C D C l}_{3}\right): \delta,-139.9--139.8(\mathrm{~m}, 1 \mathrm{~F}),-135.04--134.96(\mathrm{~m}$, 1F). IR (ATR-IR): 3416, 2969, 1599, 1548, 1467, 1368, 1330, 1265, 11043, 1039, 1008, 898, 759, 741, 711, 694, 629, $561,509 \mathrm{~cm}^{-1}$. HRMS (ESI): $\mathrm{m} / z$ calculated for $\mathrm{C}_{28} \mathrm{H}_{30} \mathrm{~F}_{2} \mathrm{NaO}_{2} \mathrm{Si}[\mathrm{M}+\mathrm{Na}]^{+}=487.1875$, found: 487.1874 .

\section{5-((5,6-diphenyl-5H-silolo[2',3':4,5] benzo[1,2-d][1,3] dioxol-5-yl)oxy)-2,5-dimethylhexan-2-ol (3t)}

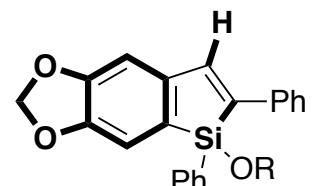

Following the General Procedure ( $0.2 \mathrm{mmol}$ scale), the titled compound was obtained as colorless oil in 94\% yield $(88.7 \mathrm{mg}) .{ }^{1} \mathbf{H}$ NMR $\left(\mathbf{4 0 0} \mathbf{~ M H z}, \mathbf{C D C l}_{3}\right): \delta 1.11(\mathrm{~s}, 3 \mathrm{H}), 1.13(\mathrm{~s}$, $3 \mathrm{H}), 1.18(\mathrm{~s}, 3 \mathrm{H}), 1.25(\mathrm{~s}, 3 \mathrm{H}), 1.51-1.57(\mathrm{~m}, 4 \mathrm{H}), 5.94(\mathrm{~d}, J=1.2 \mathrm{~Hz}, 1 \mathrm{H}), 5.98(\mathrm{~d}, J=1.2 \mathrm{~Hz}$, $1 \mathrm{H}), 6.83(\mathrm{~s}, 1 \mathrm{H}), 6.97(\mathrm{~s}, 1 \mathrm{H}), 7.17(\mathrm{tt}, J=7.1,1.7 \mathrm{~Hz}, 1 \mathrm{H}), 7.24-7.29(\mathrm{~m}, 2 \mathrm{H}), 7.32(\mathrm{~d}, J=$ $7.6 \mathrm{~Hz}, 2 \mathrm{H}), 7.37(\mathrm{tt}, J=7.3,1.5 \mathrm{~Hz}, 1 \mathrm{H}), 7.48(\mathrm{dd}, J=8.0,1.2 \mathrm{~Hz}, 2 \mathrm{H}), 7.54(\mathrm{~s}, 1 \mathrm{H}), 7.63(\mathrm{dd}, J=8.1,1.5 \mathrm{~Hz}, 2 \mathrm{H})$. 
The proton of hydroxy group was not detected. ${ }^{13} \mathbf{C}$ NMR (125 $\mathbf{~ M H z}, \mathbf{C D C l}_{3}$ ): $\delta 29.23$ (2C, overlapped), 29.24 (2C, overlapped), 29.7, 38.4, 39.0, 70.8, 76.4, 101.1, 106.6, 112.7, 126.9, 127.1, 128.1, 128.7, 130.2, 134.1, 134.3, 138.3, 141.3, 141.9, 143.0, 147.5, 149.9. IR (ATR-IR): 3445, 2970, 1597, 1542, 1467, 1429, 1385, 1367, 1282, 1233, 1172, $1103,1036,986,933,897,755,717,693,667,559 \mathrm{~cm}^{-1}$. HRMS (ESI): $m / z$ calculated for $\mathrm{C}_{29} \mathrm{H}_{32} \mathrm{NaO}_{4} \mathrm{Si}[\mathrm{M}+\mathrm{Na}]^{+}=$ 495.1962, found: 495.1959 .

1,3-dihexyl-5-((5-hydroxy-2,5-dimethylhexan-2-yl)oxy)-5,6-diphenyl-3,5-dihydrosilolo[2',3':4,5] benzo[1,2-d ]imidazol-2(1H)-one (3u)

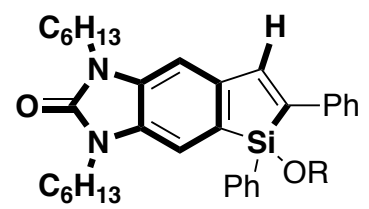

Following the General Procedure, the titled compound was obtained as yellow fluorescent viscose oil in 56\% yield (109.7 mg). ${ }^{1} \mathbf{H}$ NMR (400 MHz, $\left.\mathbf{C D C l}_{3}\right): \delta 0.84(\mathrm{t}, J=$ $6.9 \mathrm{~Hz}, 3 \mathrm{H}), 0.90(\mathrm{t}, J=6.9 \mathrm{~Hz}, 3 \mathrm{H}), 1.11(\mathrm{~s}, 3 \mathrm{H}), 1.12(\mathrm{~s}, 3 \mathrm{H}), 1.18(\mathrm{~s}, 3 \mathrm{H}), 1.25(\mathrm{~s}, 3 \mathrm{H})$, $1.26-1.43(\mathrm{~m}, 11 \mathrm{H}), 1.51-1.81(\mathrm{~m}, 10 \mathrm{H}), 3.75-3.94(\mathrm{~m}, 4 \mathrm{H}), 6.95(\mathrm{~s}, 1 \mathrm{H}), 7.12(\mathrm{~s}, 1 \mathrm{H})$, $7.18(\mathrm{tt}, J=7.3,1.5 \mathrm{~Hz}, 1 \mathrm{H}), 7.28-7.34(\mathrm{~m}, 4 \mathrm{H}), 7.39(\mathrm{tt}, J=7.3,1.5 \mathrm{~Hz}, 1 \mathrm{H}), 7.50(\mathrm{dd}, J=8.6,1.2 \mathrm{~Hz}, 2 \mathrm{H}), 7.67(\mathrm{dd}$, $J=8.1,1.5 \mathrm{~Hz}, 2 \mathrm{H}), 7.68(\mathrm{~s}, 1 \mathrm{H}) .{ }^{13} \mathbf{C}$ NMR (100 MHz, $\left.\mathbf{C D C l}_{3}\right): \delta 14.12,14.17,22.6,22.7,26.65,26.70,28.6,28.7$, 29.27 (2C, overlapped), 29.30, 29.7, 31.56, 31.61, 38.4, 39.1, 41.36, 41.40, 70.8, 76.4, 105.1, 112.2, 126.9, 127.1, 127.5, 128.2, 128.7, 129.4, 130.2, 131.7, 134.40, 134.44, 138.5, 141.5, 141.9, 142.2, 154.2. IR (ATR-IR): 2961, 2928, 2858, 1688, 1607, 1541, 1490, 1457, 1407, 1366, 1288, 1232, 1191, 1135, 1113, 1064, 1038, 1024, 911, 898, 761, 741, 727, 713, 696, 647, 562, 497, 484, 473, 459, 436, 425, $417 \mathrm{~cm}^{-1}$. HRMS (ESI): $\mathrm{m} / z$ calculated for $\mathrm{C}_{41} \mathrm{H}_{56} \mathrm{~N}_{2} \mathrm{NaO}_{3} \mathrm{Si}$ $[\mathrm{M}+\mathrm{Na}]^{+}=675.3952$, found: 675.3953 .

2,5-dimethyl-5-((6-methyl-1,2-diphenyl-1H-silolo[3,2-b]pyridin-1-yl)oxy)hexan-2-ol (3v)

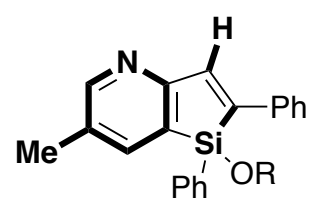

Following the General Procedure, the titled compound was obtained as colorless oil in $72 \%$ yield (95.7 mg). ${ }^{1} \mathbf{H}$ NMR (400 MHz, $\left.\mathbf{C D C l}_{3}\right): \delta 1.12(\mathrm{~s}, 3 \mathrm{H}), 1.13(\mathrm{~s}, 3 \mathrm{H}), 1.18(\mathrm{~s}, 3 \mathrm{H}), 1.24$ $(\mathrm{s}, 3 \mathrm{H}), 1.50-1.61(\mathrm{~m}, 4 \mathrm{H}), 2.28(\mathrm{~s}, 3 \mathrm{H}), 7.23(\mathrm{t}, J=7.3 \mathrm{~Hz}, 1 \mathrm{H}), 7.28-7.35(\mathrm{~m}, 4 \mathrm{H}), 7.40(\mathrm{tt}, J$ $=7.3,1.2 \mathrm{~Hz}, 1 \mathrm{H}), 7.55(\mathrm{dd}, J=7.9,1.2 \mathrm{~Hz}, 2 \mathrm{H}), 7.62(\mathrm{brs}, 1 \mathrm{H}), 7.63(\mathrm{dd}, J=7.9,1.5 \mathrm{~Hz}, 2 \mathrm{H})$,

7.79 (brs, $1 \mathrm{H}), 8.34$ (brs, $1 \mathrm{H})$. The proton of hydroxy group was not detected. ${ }^{13} \mathbf{C}$ NMR $\left(\mathbf{1 2 5} \mathbf{~ M H z}, \mathbf{C D C l}_{3}\right): \delta 18.6$, 29.2, 29.26, 29.29, 29.6, 38.4, 39.0, 70.6, 76.9, 127.4, 128.0, 128.3, 128.8, 129.6, 130.5, 131.4, 132.8, 134.3, 137.6, 140.9, 143.1, 146.9, 151.2, 163.8. IR (ATR-IR): 3385, 2968, 1561, 1531, 1490, 1428, 1369, 1264, 1103, 1072, 1038, 997, 944, 901, 776, 752, 716, 696, 596, $569 \mathrm{~cm}^{-1}$. HRMS (ESI): $\mathrm{m} / z$ calculated for $\mathrm{C}_{28} \mathrm{H}_{33} \mathrm{NNaO}_{2} \mathrm{Si}[\mathrm{M}+\mathrm{H}]^{+}=$ 444.2353, found: 444.2349 .

\section{5-((1,2-diphenyl-1H-benzo[b] silolo[2,3-d] thiophen-1-yl)oxy)-2,5-dimethylhexan-2-ol (3w)}

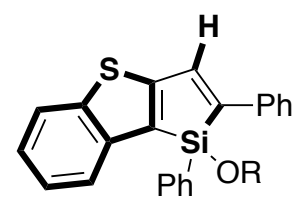

Following the General Procedure (pTLC (Hexane/EtOAc 4:1)), the titled compound was obtained as yellow oil in 50\% yield (72.9 mg). ${ }^{1} \mathbf{H}$ NMR (400 $\left.\mathbf{~ M H z}, \mathbf{C D C l}_{3}\right): \delta 1.073(\mathrm{~s}, 3 \mathrm{H})$, $1.078(\mathrm{~s}, 3 \mathrm{H}), 1.22(\mathrm{~s}, 3 \mathrm{H}), 1.26(\mathrm{~s}, 3 \mathrm{H}), 1.47-1.63(\mathrm{~m}, 4 \mathrm{H}), 7.20-7.25(\mathrm{~m}, 2 \mathrm{H}), 7.28-7.32(\mathrm{~m}$, $5 \mathrm{H}), 7.37(\mathrm{tt}, J=7.3,1.5 \mathrm{~Hz}, 1 \mathrm{H}), 7.56(\mathrm{dd}, J=7.1,1.5 \mathrm{~Hz}, 2 \mathrm{H}), 7.63(\mathrm{~s}, 1 \mathrm{H}), 7.67-7.70(\mathrm{~m}$, $3 \mathrm{H}), 7.85(\mathrm{~d}, J=7.8 \mathrm{~Hz}, 1 \mathrm{H})$. The proton of hydroxy group was not detected. ${ }^{13} \mathbf{C} \mathbf{~ N M R}\left(\mathbf{1 0 0} \mathbf{~ M H z}, \mathbf{C D C l}_{\mathbf{3}}\right): \delta 29.1$, 29.17, 29.20, 29.4, 38.5, 39.1, 70.8, 76.9, 123.1, 123.6, 124.3, 125.2, 127.1, 127.8, 128.3, 128.9, 130.5, 132.29, 132.31, 132.9, 134.4, 137.8, 141.1, 143.8, 146.1, 156.6. IR (ATR-IR): 3433, 2967, 1455, 1429, 1367, 1314, 1103, 1037, 976, 907, 871, 757, 694, 566, $522 \mathrm{~cm}^{-1}$. HRMS (ESI): $\mathrm{m} / z$ calculated for $\mathrm{C}_{30} \mathrm{H}_{32} \mathrm{NaO}_{2} \mathrm{SSi}[\mathrm{M}+\mathrm{Na}]^{+}=507.1785$, found: 507.1780 . 


\section{5-((1,2-diphenyl-4,5,6,7-tetrahydro-1H-benzo[b]silol-1-yl)oxy)-2,5-dimethylhexan-2-ol (3x)}

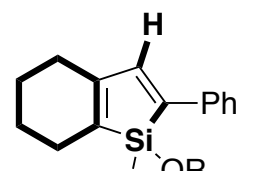

Following the General Procedure, the titled compound was obtained as colorless oil in $92 \%$ (119.6 mg). ${ }^{1} \mathbf{H}$ NMR (400 MHz, $\left.\mathbf{C D C l}_{3}\right): \delta 1.10(\mathrm{~s}, 3 \mathrm{H}), 1.12(\mathrm{~s}, 3 \mathrm{H}), 1.22(\mathrm{~s}, 3 \mathrm{H}), 1.28(\mathrm{~s}, 3 \mathrm{H})$, 1.48-1.76 (m, 8H), 2.08-2.39 (m, 4H), 7.01 (s, $1 \mathrm{H}), 7.09(\mathrm{dt}, J=7.3,1.7 \mathrm{~Hz}, 1 \mathrm{H}), 7.20(\mathrm{dd}, J=7.3$, $1.7 \mathrm{~Hz}, 2 \mathrm{H}), 7.29-7.35(\mathrm{~m}, 3 \mathrm{H}), 7.39(\mathrm{dd}, J=7.6,1.2 \mathrm{~Hz}, 2 \mathrm{H}), 7.63(\mathrm{dd}, J=7.6,1.7 \mathrm{~Hz}, 2 \mathrm{H})$. The proton of hydroxy group was not detected. $\left.{ }^{13} \mathbf{C} \mathbf{~ N M R ~ ( 1 0 0 ~} \mathbf{~ M H z}, \mathbf{C D C l}_{3}\right): \delta 22.9,23.1,25.0,28.8,29.1,29.2$ (2C, overlapped), 29.4, 38.5, 39.1, 70.8, 75.7, 126.6, 126.8, 128.1, 128.5, 129.8, 133.0, 134.2, 134.4, 138.4, 139.6, 141.8, 150.3. IR (ATR-IR): 3409, 2969, 2927, 1594, 1524, 1488, 1429, 1366, 1218, 1104, 1038, 1014, 906, 878, 755, 693, 635, $559 \mathrm{~cm}^{-1}$. HRMS (ESI): $m / z$ calculated for $\mathrm{C}_{28} \mathrm{H}_{36} \mathrm{NaO}_{2} \mathrm{Si}[\mathrm{M}+\mathrm{Na}]^{+}=455.2377$, found: 455.2375 .

\section{5-(((6bS,8aS,12bR)-4-methoxy-8a-methyl-9,10-diphenyl-1,2,6b,7,8,8a,9,12,12a,12b-decahydronaphtho[2',1'} $: 4,5]$ indeno[1,2-b]silol-9-yl)oxy)-2,5-dimethylhexan-2-ol (3y)

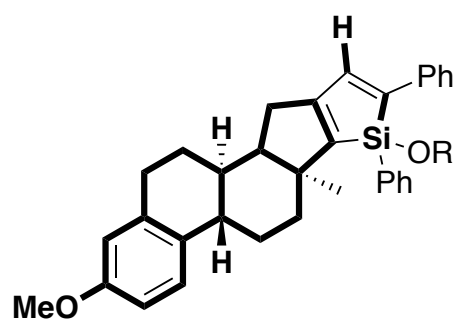

Following the General Procedure $\left(0.2 \mathrm{mmol}\right.$ scale, reaction at $\left.60^{\circ} \mathrm{C}\right)$, the titled compound (a mixture of diastereomers; 3:1) was obtained as colorless oil in 67\% (41.5 mg). ${ }^{1} \mathbf{H}$ NMR (400 MHz, $\mathbf{C D C l}_{3}$ ): $\delta 0.51$ (s, 3H; major), 1.07 (s, 3H; minor), $1.13(\mathrm{~s}, 3 \mathrm{H}), 1.14(\mathrm{~s}, 3 \mathrm{H}), 1.23-1.26(\mathrm{~m}, 3 \mathrm{H}), 1.32-1.35(\mathrm{~m}, 4 \mathrm{H}), 1.43-2.03(\mathrm{~m}$, $11 \mathrm{H}), 2.17-2.37(\mathrm{~m}, 3 \mathrm{H}), 2.39-2.47(\mathrm{~m}, 1 \mathrm{H}), 2.83-2.97(\mathrm{~m}, 2 \mathrm{H}), 3.77(\mathrm{~s}, 3 \mathrm{H}), 6.63$ (s, $1 \mathrm{H}$; minor), $6.64(\mathrm{~s}, 1 \mathrm{H}$; major), 6.67-6.72 (m, $1 \mathrm{H}), 7.11-7.23(\mathrm{~m}, 5 \mathrm{H})$, 7.29-7.39 (m, 5H), 7.66 (dd, $J=7.8,1.5 \mathrm{~Hz}, 2 \mathrm{H}$; major), 7.70 (dd, $J=7.8,1.5 \mathrm{~Hz}, 2 \mathrm{H}$; minor). ${ }^{13} \mathbf{C}$ NMR (100 MHz, $\mathbf{C D C l}_{3}$ ): $\delta 17.9$ (major), 18.7 (minor), 26.5 (minor), 26.8 (major), 28.4 (minor), 28.5 (major), 29.0 (minor), 29.1 (major), 29.21 (major), 29.24 (minor), 29.3, 29.4, 29.85 (minor), 29.93 (major), 33.2 (minor), 33.5 (major), 36.5 (minor), 37.5 (major), 37.7 (major), 37.8 (minor), 38.55 (major), 38.64 (minor), 39.1, 44.3 (minor), 44.5 (major), 48.1 (minor), 48.7 (major), 55.3, 58.6 (major), 59.6 (minor), 70.9, 75.85 (major), 75.89 (minor), 11.50 (minor), 111.54 (major), 114.0, 126.1 (minor), 126.2 (major), 126.8, 126.85 (2C, overlapped, minor), 126.93 (2C, overlapped, major), 128.0 (2C, overlapped, major), 128.1 (2C, overlapped, minor), 128.6 (2C, overlapped), 129.8, 133.1 (major), 133.2 (minor), 134.17 (2C overlapped, minor), 134.21 (2C overlapped, major), 134.5 (major), 134.7 (minor), 136.07 (major), 136.13 (minor), 138.01 (minor), 138.05 (major), 138.7 (major), 139.0 (minor), 143.3 (minor), 143.7 (major) 152.0, 157.5 (minor), 157.6 (major), 160.6 (major), 161.4 (minor). IR (ATR-IR): 3441, 2968, 2927, 1608, 1497, 1429, 1367, 1281, 1247, 1135, 1104, 1041, 908, 800, 755, 696, 667, 613, $535 \mathrm{~cm}^{-1}$. HRMS (ESI): $m / z$ calculated for $\mathrm{C}_{41} \mathrm{H}_{50} \mathrm{NaO}_{3} \mathrm{Si}[\mathrm{M}+\mathrm{Na}]^{+}=641.3421$, found: 641.3418 . 


\section{2-4. Transformation of Benzosilole (Scheme 3)}

\section{1-(4-methoxyphenyl)-1,2-diphenyl-1H-benzo[b] silole (4)}

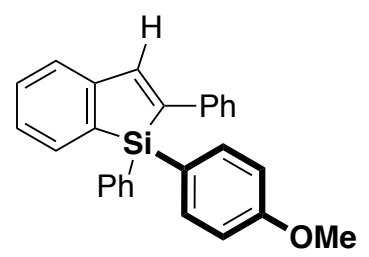

To a solution of $3 \mathrm{a}(40.2 \mathrm{mg}, 0.093 \mathrm{mmol}, 1.0$ equiv) in THF $(2.0 \mathrm{ml})$ in a heat gun-dried Schlenk tube was added 4-methoxyphenyllithium $(0.206 \mathrm{mmol}, 2.2$ equiv, prepared from 1-bromo-4-methoxybenzene and ${ }^{t} \mathrm{BuLi}$ at $-78{ }^{\circ} \mathrm{C}$ ) at $0{ }^{\circ} \mathrm{C}$. The resultant mixture was warmed to $80^{\circ} \mathrm{C}$, and stirred for $72 \mathrm{~h}$. The reaction was cooled to $\mathrm{rt}$, and quenched with sat.

$\mathrm{NH}_{4} \mathrm{Cl}$ aq. followed by extraction with EtOAc $x$ 3. The combined organic layer was dried over $\mathrm{Na}_{2} \mathrm{SO}_{4}$, filtered and concentrated in vacuo. Purification by MPLC (eluent: hexane/EtOAc 9:1) afforded the product 4 as colorless oil in 93\% (34.0 mg). ${ }^{1} \mathbf{H}$ NMR (500 $\left.\mathbf{M H z}, \mathbf{C D C l}_{3}\right): \delta 3.78(\mathrm{~s}, 3 \mathrm{H}), 6.89(\mathrm{~d}, \mathrm{~J}=8.5 \mathrm{~Hz}, 2 \mathrm{H})$, 7.17-7.22 (m, 2H), 7.25-7.28 (m, 2H), 7.31-7.34 (m, 2H), $7.37(\mathrm{~d}, J=4.0 \mathrm{~Hz}, 2 \mathrm{H}), 7.40(\mathrm{tt}, J=7.6,1.5 \mathrm{~Hz}, 1 \mathrm{H}), 7.50$ $(\mathrm{dd}, J=7.0,1.5 \mathrm{~Hz}, 2 \mathrm{H}), 7.57(\mathrm{~d}, J=8.5 \mathrm{~Hz}, 2 \mathrm{H}), 7.60(\mathrm{dd}, J=6.9,0.9 \mathrm{~Hz}, 1 \mathrm{H}), 7.65(\mathrm{dd}, J=7.9,1.5 \mathrm{~Hz}, 2 \mathrm{H}), 7.73(\mathrm{~s}$, $1 \mathrm{H}) .{ }^{13} \mathbf{C}$ NMR (125 MHz, $\mathbf{C D C l}_{3}$ ): $\delta$ 55.2, 114.3, 122.7, 124.9, 127.2, 127.32, 127.35, 128.3, 128.8, 130.2, 130.6, 132.7, 133.0, 135.8, 136.7, 137.4, 139.2, 134.3, 143.8, 149.7, 161.5. IR (ATR-IR): 2972, 1593, 1561, 1501, 1429, 1368, $1280,1248,1181,1111,1027,961,898,866,824,797,765,733,693,674,639,601,554 \mathrm{~cm}^{-1}$. HRMS (APCI): $m / z$ calculated for $\mathrm{C}_{27} \mathrm{H}_{23} \mathrm{OSi}[\mathrm{M}+\mathrm{H}]^{+}=391.1513$, found: 391.1511 .

\section{1,2-diphenyl-1-(phenylethynyl)-1H-benzo $[\boldsymbol{b}]$ silole (5)}

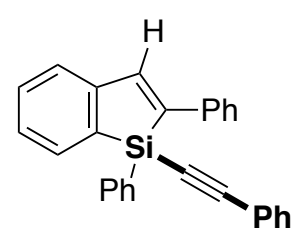

To a solution of $3 \mathrm{a}(85.7 \mathrm{mg}, 0.20 \mathrm{mmol}, 1.0$ equiv $)$ in THF $(2.0 \mathrm{ml})$ in a heat gun-dried Schlenk tube was added $\mathrm{BF}_{3} \cdot \mathrm{Et}_{2} \mathrm{O}(100 \mu \mathrm{L}, 0.80 \mathrm{mmol}, 4.0$ equiv $)$ at $0{ }^{\circ} \mathrm{C}$. The reaction mixture was warmed to rt and stirred for $20 \mathrm{~h}$. Removal of the solvent in vacuo gave slightly reddish oil. This crude material was dissoved in THF $(2.0 \mathrm{~mL})$ and cooled to $0{ }^{\circ} \mathrm{C}$. To the solution was added phenylethynyllithium ( $1.00 \mathrm{mmol}, 5.0$ equiv, prepared from ethynylbezene and ${ }^{\mathrm{n}} \mathrm{BuLi}$ at $-78^{\circ} \mathrm{C}$ ). The reaction mixture was warmed to $60{ }^{\circ} \mathrm{C}$ and stirred for $12 \mathrm{~h}$. The reaction was quenched with sat. $\mathrm{NH}_{4} \mathrm{Cl}$ aq. followed by extraction with EtOAc x 3. The combined organic layer was dried over $\mathrm{Na}_{2} \mathrm{SO}_{4}$, filtered and concentrated in vacuo to give brown oil. Purification with MPLC (eluent: hexane/EtOAc 9:1) and $\mathrm{GPC}\left(\mathrm{CHCl}_{3}\right)$ afforded the product 5 as slightly reddish oil in $68 \%$ yield $(52.0 \mathrm{mg}) .{ }^{1} \mathbf{H} \mathbf{N M R}\left(\mathbf{5 0 0} \mathbf{M H z}, \mathbf{C D C l}_{3}\right): \delta 7.20(\mathrm{tt}, J=7.3$, $1.5 \mathrm{~Hz}, 1 \mathrm{H}), 7.23(\mathrm{dd}, J=7.2,1.5 \mathrm{~Hz}, 1 \mathrm{H}), 7.28-7.33(\mathrm{~m}, 5 \mathrm{H}), 7.34-7.36(\mathrm{~m}, 3 \mathrm{H}), 7.37-7.42(\mathrm{~m}, 2 \mathrm{H}), 7.54(\mathrm{dd}, J=6.4$, $1.5 \mathrm{~Hz}, 2 \mathrm{H}), 7.62-7.64(\mathrm{~m}, 3 \mathrm{H}), 7.72(\mathrm{~s}, 1 \mathrm{H}), 7.77$ (dd, $J=6.4,1.5 \mathrm{~Hz}, 2 \mathrm{H}) .{ }^{13} \mathbf{C}$ NMR (125 $\left.\mathbf{M H z}, \mathbf{C D C l}_{3}\right): \delta 86.4$, 109.7, 122.6, 124.9, 127.2 (2C, overlapped), 127.5, 127.7, 128.4 (4C, overlapped), 128.8 (2C, overlapped), 129.4, 130.6, 131.1, 131.4, 132.5 (2C, overlapped), 133.1, 134.4, 135.1 (2C, overlapped), 138.2, 141.7, 143.8, 149.8. IR (ATR-IR): 3051, 2154, 1595, 1488, 1440, 1428, 1219, 1111, 1060, 1027, 946, 898, 836, 756, 740, 715, 688, 637, 600, $552,537 \mathrm{~cm}^{-1}$. HRMS (APCI): $m / z$ calculated for $\mathrm{C}_{28} \mathrm{H}_{21} \mathrm{Si}[\mathrm{M}+\mathrm{H}]^{+}=385.1407$, found: 385.1402 .

\section{1,2-diphenyl-1H-benzo[b] silol-1-ol (6)}

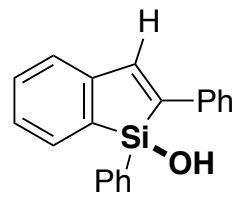

To a solution of 3a (41.1 mg, $0.096 \mathrm{mmol}, 1.0$ equiv) in THF (1.0 ml) in a heat gun-dried Schlenk tube was added $\mathrm{BF}_{3} \cdot \mathrm{Et}_{2} \mathrm{O}\left(24 \mu \mathrm{L}, 0.192 \mathrm{mmol}, 2.0\right.$ equiv) at $0{ }^{\circ} \mathrm{C}$. The reaction mixture was warmed to rt and stirred for $20 \mathrm{~h}$. Removal of the solvent gave slightly yellow oil. This crude material was loaded on the silica gel column for MPLC and eluted with a mixture of hexane $/ \mathrm{CH}_{2} \mathrm{Cl}_{2}$ (1:1) afforded the product 6 as colorless oil in 98\% yield $(28.4 \mathrm{mg}) .{ }^{1} \mathbf{H} \mathbf{N M R}\left(\mathbf{4 0 0} \mathbf{~ M H z}, \mathbf{C D C l}_{3}\right): \delta 2.48(\mathrm{brs}, 1 \mathrm{H})$, $7.22(\mathrm{dd}, J=7.1,0.7 \mathrm{~Hz}, 2 \mathrm{H}), 7.27-7.38(\mathrm{~m}, 6 \mathrm{H}), 7.42(\mathrm{tt}, J=7.3,1.5 \mathrm{~Hz}, 1 \mathrm{H}), 7.52-7.56(\mathrm{~m}, 3 \mathrm{H}), 7.67(\mathrm{~s}, 1 \mathrm{H}), 7.71$ $(\mathrm{dd}, J=8.0,1.5 \mathrm{~Hz}, 2 \mathrm{H}) .{ }^{13} \mathbf{C}$ NMR (100 $\left.\mathbf{M H z}, \mathbf{C D C l}_{3}\right): \delta 124.8,126.9,127.6,127.9,128.3,128.9,130.8,131.5$, 132.66, 132.74, 133.9, 134.5, 138.0, 141.6, 143.3, 148.4. IR (ATR-IR): 3593, 3388, 3054, 2925, 1711, 1591, 1490, 
1443, 1429, 1278, 1117, 1070, 901, 848, 766, 741, 716, 697, 604, 552, $517 \mathrm{~cm}^{-1}$. HRMS (APCI): $m / z$ calculated for $\mathrm{C}_{20} \mathrm{H}_{17} \mathrm{OSi}[\mathrm{M}+\mathrm{H}]^{+}=301.1043$, found: 301.1053 .

\section{1,2-diphenyl-1H-benzo[b] silole (7)}

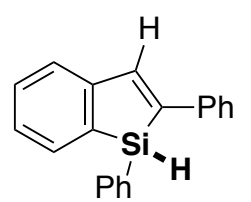

To a solution of 3a $(224.5 \mathrm{mg}, 0.52 \mathrm{mmol}, 1.0$ equiv) in THF $(8.0 \mathrm{ml})$ in a heat gun-dried Schlenk tube was added DIBAL ( $1.03 \mathrm{M}$ in $\mathrm{n}$-hexane, $2.03 \mathrm{~mL}, 2.1 \mathrm{mmol}, 4.0$ equiv) at $0{ }^{\circ} \mathrm{C}$. The reaction mixture was warmed to $80^{\circ} \mathrm{C}$ and stirred for $20 \mathrm{~h}$. The reaction was cooled to rt, and quenched by sat. $\mathrm{NH}_{4} \mathrm{Cl}$ aq. and subsequently acidified with $1.0 \mathrm{M} \mathrm{HCl}$ aq. The organic layer was separated, and the aqueous phase was extracted with ether $\mathrm{x} 3$. The combined organic layer was dried over $\mathrm{Na}_{2} \mathrm{SO}_{4}$, filtered, and concentrated under reduced pressure. The crude product was purified with MPLC (eluent: hexane $/ \mathrm{CH}_{2} \mathrm{Cl}_{2}$ 8:1) to afford 7 as white solid in 79\% yield (117.1 mg). Mp.: 119.1-120.3 ${ }^{\circ} \mathrm{C}$ (hexane). ${ }^{1} \mathbf{H}$ NMR (400 $\left.\mathbf{~ M H z}, \mathbf{C D C l}_{3}\right): \delta 5.41$ $(\mathrm{s}, 1 \mathrm{H}), 7.19-7.24(\mathrm{~m}, 2 \mathrm{H}), 7.28-7.42(\mathrm{~m}, 7 \mathrm{H}), 7.49(\mathrm{dd}, J=7.1,1.5 \mathrm{~Hz}, 2 \mathrm{H}), 7.59(\mathrm{~d}, J=6.6 \mathrm{~Hz}, 1 \mathrm{H}), 7.62(\mathrm{dd}, J=$ 6.6, $1.5 \mathrm{~Hz}, 2 \mathrm{H}), 7.70(\mathrm{~s}, 1 \mathrm{H}) .{ }^{13} \mathbf{C}$ NMR (125 $\left.\mathbf{M H z}, \mathbf{C D C l}_{3}\right): \delta 124.8,127.2,127.4,127.5,128.5,128.8,130.5,130.9$, 131.2, 133.5, 133.8, 135.6, 138.6, 142.1, 143.9, 150.4. IR (ATR-IR): 3045, 2159, 1487, 1428, 1261, 1112, 1057, 947, 904, 799, 762, 744, 733, 711, 687, 545, $523 \mathrm{~cm}^{-1}$. HRMS (APCI): $\mathrm{m} / z$ calculated for $\mathrm{C}_{20} \mathrm{H}_{17} \mathrm{Si}[\mathrm{M}+\mathrm{H}]^{+}=285.1094$, found: 285.1097. Anal.: Calcd: C, 84.46; H, 5.67. Found: C 84.40; H, 6.01.

\section{1-methyl-1,2-diphenyl-1H-benzo[b]silole (8)}<smiles>C[Si]1(c2ccccc2)C(=Cc2ccccc2)c2ccccc21</smiles>

To a solution of $7(28.4 \mathrm{mg}, 0.10 \mathrm{mmol}, 1.0$ equiv $)$ in THF $(1.0 \mathrm{ml})$ in a heat gun-dried Schlenk tube was added TMPLi $\left(0.200 \mathrm{mmol}, 2.0\right.$ equiv. prepared from TMP and $\left.{ }^{\mathrm{n}} \mathrm{BuLi}\right)$ in THF $(1.0 \mathrm{~mL})$ at $-78^{\circ} \mathrm{C}$. After stirring at $-78{ }^{\circ} \mathrm{C}$ for $15 \mathrm{~min}$, iodomethane $(62.3 \mu \mathrm{L}, 1.0 \mathrm{mmol}, 10.0$ equiv) was added. The resulting mixture was quenched by sat. $\mathrm{NH}_{4} \mathrm{Cl}$ aq. The organic layer was separated, and the aqueous phase was extracted with EtOAc $x$ 3. The combined organic layer was dried over $\mathrm{Na}_{2} \mathrm{SO}_{4}$, filtered, and concentrated under reduced pressure. The crude product was purified with MPLC (eluent: hexane) to afford the titled compound as colorless solid in 19\% yield $(5.5 \mathrm{mg}) .{ }^{1} \mathrm{H}$ NMR spectrum was in agreement with the reference. ${ }^{18}{ }^{1} \mathbf{H}$ NMR (400 MHz, $\left.\mathbf{C D C l}_{3}\right): \delta 0.78(\mathrm{~s}, 3 \mathrm{H}), 7.18-7.23(\mathrm{~m}, 2 \mathrm{H}), 7.27-7.40(\mathrm{~m}, 7 \mathrm{H}), 7.46(\mathrm{dd}, J=8.0,1.2 \mathrm{~Hz}, 2 \mathrm{H}), 7.51$ $(\mathrm{dd}, J=7.1,0.6 \mathrm{~Hz}, 1 \mathrm{H}), 7.58(\mathrm{dd}, J=8.0,1.5 \mathrm{~Hz}, 2 \mathrm{H}), 7.68(\mathrm{~s}, 1 \mathrm{H})$. HRMS (APCI): $m / z$ calculated for $\mathrm{C}_{21} \mathrm{H}_{19} \mathrm{Si}$ $[\mathrm{M}+\mathrm{H}]^{+}=299.1251$, found: 298.1263 .

\section{5-((2-([1,1'-biphenyl]-2-yl)-1-phenyl-1 H-benzo[b] silol-1-yl)oxy)-2,5-dimethylhexan-2-ol (3z)}

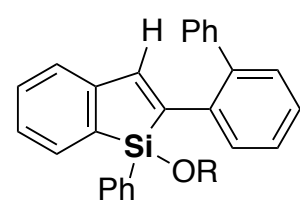

$\mathrm{Ph}$

Following the General Procedure for Table 2, the titled compound was obtained as colorless oil in 99\% yield (400.0 mg; $0.804 \mathrm{mmol} \mathrm{scale).}{ }^{1} \mathbf{H}$ NMR (400 $\left.\mathbf{~ M H z}, \mathbf{C D C l}_{3}\right): \delta 1.10$ (s, 3H), $1.11(\mathrm{~s}, 3 \mathrm{H}), 1.27\left(\mathrm{~s}, 4 \mathrm{H}\right.$; OH was overlapped with $\left.\mathrm{CH}_{3}\right), 1.29(\mathrm{~s}, 3 \mathrm{H}), 1.55-1.65(\mathrm{~m}, 4 \mathrm{H}), 6.59$

$(\mathrm{s}, 1 \mathrm{H}), 6.80(\mathrm{~d}, J=7.3 \mathrm{~Hz}, 1 \mathrm{H}), 7.08-7.34(\mathrm{~m}, 13 \mathrm{H}), 7,48(\mathrm{~d}, J=6.7 \mathrm{~Hz}, 1 \mathrm{H}), 7.52(\mathrm{dd}, J=6.6$, $2.0 \mathrm{~Hz}, 1 \mathrm{H}), 7.58-7.61(\mathrm{~m}, 2 \mathrm{H}) .{ }^{13} \mathbf{C}$ NMR (100 MHz, $\left.\mathbf{C D C l}_{3}\right): \delta 29.2,29.3,29.5,29.7,38.4,39.0,70.7,76.4,124.7$, 126.5 , 126.7, 127.2, 127.5, 128.0, 128.3, 129.4, 130.0, 130.1, 130.8, 131.3, 132.6, 134.3, 134.4, 134.5, 137.3, 140.9, 141.9, 142.9, 147.7, 148.1. IR (ATR-IR): 2967, 2181, 1590, 1473, 1440, 1367, 1279, 1112, 1065, 1036, 944, 907, 806, $741,715,698,606,573,518 \mathrm{~cm}^{-1}$. HRMS (ESI): $\mathrm{m} / z$ calculated for $\mathrm{C}_{34} \mathrm{H}_{36} \mathrm{NaO}_{2} \mathrm{Si}[\mathrm{M}+\mathrm{Na}]^{+}=527.2377$, found: 527.2366 .

(18) Matsuda, T.; Yamaguchi, Y.; Shigeno, M.; Sato, S.; Murakami, M. Chem. Commun. 2011, 47, 8697. S-20 
2-([1,1'-biphenyl]-2-yl)-1-phenyl-1H-benzo[b]silole (9)

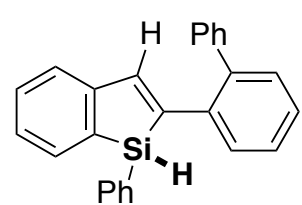

To a solution of $3 \mathbf{z}(400.0 \mathrm{mg}, 0.79 \mathrm{mmol}, 1.0$ equiv) in THF $(12.0 \mathrm{~mL})$ in a heat gun-dried Schlenk tube was added DIBAL ( $1.03 \mathrm{M}$ in n-hexane, $3.08 \mathrm{~mL}, 3.2 \mathrm{mmol}, 4.0$ equiv) at $0{ }^{\circ} \mathrm{C}$.

The reaction mixture was warmed to $80^{\circ} \mathrm{C}$ and stirred for $20 \mathrm{~h}$. The reaction was cooled to rt, and quenched by sat. $\mathrm{NH}_{4} \mathrm{Cl}$ aq. followed by acidification with $1.0 \mathrm{M} \mathrm{HCl}$ aq. The organic layer was separated, and the aqueous phase was extracted with ether $\mathrm{x} 3$. The combined organic layer was dried over $\mathrm{Na}_{2} \mathrm{SO}_{4}$, filtered, and concentrated under reduced pressure. The crude product was purified with MPLC (eluent: hexane $\left./ \mathrm{CH}_{2} \mathrm{Cl}_{2} 8: 1\right)$ to afford $\mathbf{9}$ as colorless oil in $87 \%$ yield $(247.0 \mathrm{mg}) .{ }^{1} \mathbf{H} \mathbf{N M R}\left(400 \mathbf{~ M H z}, \mathbf{C D C l}_{3}\right): \delta 3.93(\mathrm{~s}, 1 \mathrm{H})$, $7.15(\mathrm{td}, J=7.3,1.2 \mathrm{~Hz}, 1 \mathrm{H}), 7.18-7.22(\mathrm{~m}, 2 \mathrm{H}), 7.24-7.36(\mathrm{~m}, 14 \mathrm{H}), 7.42-7.46(\mathrm{~m}, 2 \mathrm{H}) .{ }^{13} \mathbf{C}$ NMR (100 MHz, $\left.\mathbf{C D C l}_{3}\right): \delta 124.5,127.06,127.09,127.4,127.7,128.1,128.3,128.5,129.96,129.98,130.5,130.6,131.2,133.2,135.1$, 135.3, 139.0, 141.1, 142.0, 144.3, 147.1, 149.9. IR (ATR-IR): 3055, 2153, 1588, 1471, 1439, 1429, 1277, 1190, 1111, $1008,950,904,802,762,736,719,696,607,564,518 \mathrm{~cm}^{-1}$. HRMS (ESI): $\mathrm{m} / z$ calculated for $\mathrm{C}_{26} \mathrm{H}_{20} \mathrm{AgSi}[\mathrm{M}+\mathrm{Ag}]^{+}=$ 467.0380, found: 467.0370 .

\section{9-phenyl-9H-dibenzo $[c, e]$ benzo $[4,5]$ silolo $[1,2-a]$ siline $(10)$}

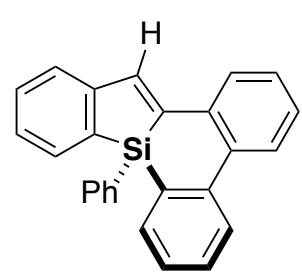

To a solution of 9 ( $36.1 \mathrm{mg}, 0.10 \mathrm{mmol}, 1.0$ equiv) and 2,6-lutidine ( $23 \mu \mathrm{L}, 0.20 \mathrm{mmol}, 2.0$ equiv) in $\mathrm{CH}_{2} \mathrm{Cl}_{2}(1.0 \mathrm{~mL})$ in a heat gun-dried Schlenk tube was added a $\mathrm{CH}_{2} \mathrm{Cl}_{2}(1.0 \mathrm{~mL})$ solution of $\mathrm{Ph}_{3} \mathrm{CB}\left(\mathrm{C}_{6} \mathrm{~F}_{5}\right)_{3}(184.5 \mathrm{mg}, 0.20 \mathrm{mmol}, 2.0$ equiv) via cannula at room temperature. The reaction mixture was stirred for $16 \mathrm{~h}$. The reaction was quenched by water. The organic layer was separated, and the aqueous phase was extracted with AcOEt x 3. The combined organic layer was dried over $\mathrm{Na}_{2} \mathrm{SO}_{4}$, filtered, and concentrated under reduced pressure. Purification with MPLC (eluent: hexane/AcOEt 10:1) and GPC $\left(\mathrm{CHCl}_{3}\right)$ afford 10 as white solid in $77 \%$ yield $(27.7 \mathrm{mg})$. Mp.: 120.1-121.7 ${ }^{\circ} \mathrm{C}$ (hexane). ${ }^{1} \mathbf{H}$ NMR (400 MHz, $\left.\mathbf{C D C l}_{3}\right): \delta 7.18(\mathrm{~s}, 1 \mathrm{H}), 7.21-7.45(\mathrm{~m}, 12 \mathrm{H}), 7.52(\mathrm{td}, J=7.7,1.5 \mathrm{~Hz}$, $1 \mathrm{H}), 7.79(\mathrm{dd}, J=7.2,1.5 \mathrm{~Hz}, 1 \mathrm{H}), 7.82(\mathrm{~d}, J=7.1 \mathrm{~Hz}, 1 \mathrm{H}), 7.85-7.89(\mathrm{~m}, 2 \mathrm{H}) .{ }^{13} \mathbf{C}$ NMR $\left(125 \mathbf{M H z}, \mathbf{C D C l}_{3}\right): \delta$ 125.4, 125.6, 126.8, 127.1, 127.7, 127.9, 128.2, 128.45, 128.53, 130.4, 130.5, 131.0, 131.2, 131.8, 134.5, 135.1, 135.2, 135.9, 137.2, 137.8, 141.1, 145.9, 146.5, 152.7. ${ }^{29}$ Si NMR (79.5 MHz, $\mathbf{C D C l}_{3}$ ): -18.6. IR (ATR-IR): 2971, 2030, 1586, 1437, 1365, 1241, 1191, 1111, 1008, 950, 855, 761, 728, 700, 575, 545, $511 \mathrm{~cm}^{-1}$. HRMS (ESI): $m / z$ calculated for $\mathrm{C}_{26} \mathrm{H}_{18} \mathrm{AgSi}[\mathrm{M}+\mathrm{Ag}]^{+}=465.0223$, found: 465.0221. Anal.: Calcd: C, 87.11; H, 5.06. Found: C 87.27; H, 5.28. CCDC number for the information on X-ray analysis: 2046045. 


\section{2-5. Hydrosilylation for Other Silacycles (Table 3)}

\section{4-((1,2-diphenyl-1H-naphtho[1,8-bc] silin-1-yl)oxy)-2,4-dimethylpentan-2-ol (11)}

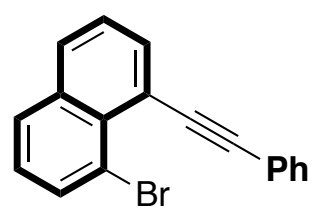

1) ${ }^{t} \mathrm{BuLi}$ (2.0 equiv), $-78^{\circ} \mathrm{C}, 15 \mathrm{~min}$

2) $\mathbf{1 b}$ (2.0 equiv), $-78^{\circ} \mathrm{C}, 15 \mathrm{~min}$; then $\mathrm{rt}, 1 \mathrm{~h}$

THF (0.04 M)

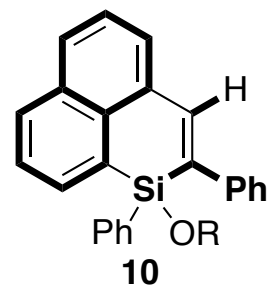

R:

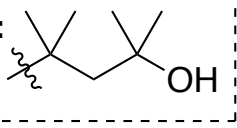

1-Bromo-8-(phenylethynyl)naphthalene (92.2 mg, $0.30 \mathrm{mmol}, 1.0$ equiv) and anhydrous THF (7.5 mL) were added to a heat gun-dried Schlenk tube. To the tube was added ${ }^{t} \mathrm{BuLi}\left(1.48 \mathrm{M}\right.$ in ${ }^{\mathrm{n}}$ pentane, $0.60 \mathrm{mmol}, 2.0$ equiv) slowly at $78^{\circ} \mathrm{C}$ and the mixture was stirred for $15 \mathrm{~min}$ before $\mathbf{1 b}(142 \mathrm{mg}, 0.60 \mathrm{mmol}, 2.0$ equiv) was added. After stirring for 15 min at $-78{ }^{\circ} \mathrm{C}$, the resultant mixture was warmed to rt slowlly and stirred for $1 \mathrm{~h}$. The reaction was quenched with sat. $\mathrm{NH}_{4} \mathrm{Cl}$ aq. followed by extraction with EtOAc $x$ 3. The combined organic layer was dried over $\mathrm{Na}_{2} \mathrm{SO}_{4}$, filtered and concentrated in vacuo. Purification with MPLC (eluent: hexane/EtOAc 4:1) afforded the product as colorless oil in 76\% yield (106.0 mg). ${ }^{1} \mathbf{H}$ NMR (500 MHz, $\mathbf{C D C l}_{3}$ ): $\delta 1.10$ (s, 3H), 1.14 (s, 3H), 1.21 (s, 3H), 1.27 (s, $\left.3 \mathrm{H}\right), 1.64$ (s, $2 \mathrm{H}$ ), 4.22 (brs, $1 \mathrm{H}), 7.22(\mathrm{tt}, J=7.3,1.2 \mathrm{~Hz}, 1 \mathrm{H}), 7.28-7.33(\mathrm{~m}, 5 \mathrm{H}), 7.52-7.57(\mathrm{~m}, 4 \mathrm{H}), 7.63-7.65(\mathrm{~m}, 3 \mathrm{H}), 7.84(\mathrm{dd}$, $J=8.1,1.0 \mathrm{~Hz}, 1 \mathrm{H}), 7.95$ (s, 1H), 7.95-7.99 (m, 2H). ${ }^{13} \mathbf{C}$ NMR (125 MHz, $\mathbf{C D C l}_{3}$ ): $\delta 31.9$ (2C, overlapped), 32.1 (2C, overlapped), 54.3, 71.3, 79.0, 125.98, 126.03, 127.2, 127.3, 128.2, 128.7, 129.8, 130.2, 131.4, 132.4, 132.87,

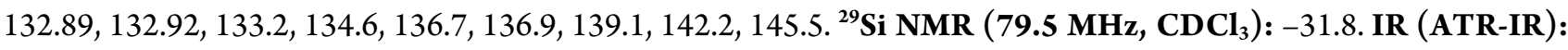
3519, 2968, 1557, 1493, 1428, 1367, 1166, 1108, 986, 911, 824, 777, 757, 696, 674, 583, 559, 538, $517 \mathrm{~cm}^{-1}$. HRMS $^{-13}$ (ESI): $m / z$ calculated for $\mathrm{C}_{31} \mathrm{H}_{32} \mathrm{NaO}_{2} \mathrm{Si}[\mathrm{M}+\mathrm{Na}]^{+}=487.2064$, found: 487.2064.

\section{1,2-diphenyl-1H-naphtho $[1,8-b c]$ siline (11')}

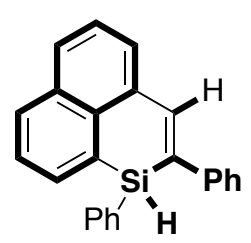

Following the procedure for the synthesis of 7, the titled compound was obtained as white solid in $86 \%$ yield $\left(65.5 \mathrm{mg}\right.$ ) from 10 (106.0 mg, 0.228 mmol). Mp.: $119.0-119.7^{\circ} \mathrm{C}$ (hexane). ${ }^{1} \mathbf{H}$ NMR (400 MHz, $\left.\mathbf{C D C l}_{3}\right): \delta 5.77(\mathrm{~s}, 1 \mathrm{H}), 7.24(\mathrm{dd}, J=7.3,1.2 \mathrm{~Hz}, 1 \mathrm{H}), 7.27-7.35(\mathrm{~m}, 5 \mathrm{H}), 7.49-7.59$ $(\mathrm{m}, 7 \mathrm{H}), 7.79(\mathrm{tt}, J=8.2,1.2 \mathrm{~Hz}, 1 \mathrm{H}), 7.86(\mathrm{~d}, J=6.1 \mathrm{~Hz}, 1 \mathrm{H}), 7.87(\mathrm{~s}, 1 \mathrm{H}), 7.93(\mathrm{dd}, J=7.9,1.2$ $\mathrm{Hz}, 1 \mathrm{H}) .{ }^{13} \mathbf{C} \mathbf{N M R}\left(\mathbf{1 2 5} \mathbf{M H z}, \mathbf{C D C l}_{3}\right): \delta 125.9,126.1,126.6,127.2,128.2,128.8,128.9,129.9$, 130.1, 131.1, 132.0, 133.1, 133.2, 134.3, 135.1, 135.4, 135.6, 136.7, 142.6, 144.7. IR (ATR-IR): 3054, 2126, 1716, $1559,1493,1445,1427,1337,1171,1108,1030,978,916,892,829,816,797,773,760,729,691,642,587,547,531$ $\mathrm{cm}^{-1}$. HRMS (APCI): $m / z$ calculated for $\mathrm{C}_{24} \mathrm{H}_{19} \mathrm{Si}[\mathrm{M}+\mathrm{H}]^{+}=335.1251$, found: 335.1242. Anal.: Calcd: $\mathrm{C}$, 86.18; $\mathrm{H}$, 5.42. Found: C 86.14; H, 5.66. CCDC number for the information on X-ray analysis: 2021313.

4-((2-mesityl-1-phenyl-1 H-naphtho[1,8-bc] silin-1-yl)oxy)-2,4-dimethylpentan-2-ol (12)

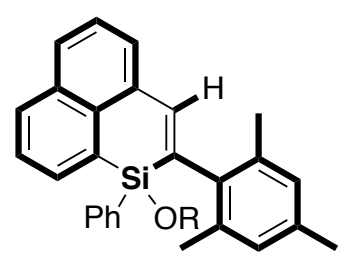

Following the procedure of the synthesis of $\mathbf{1 0}(0.18 \mathrm{mmol}$ scale $)$, the titled compound was obtained as colorless oil in 52\% yield (47.6 mg). ${ }^{1} \mathbf{H}$ NMR (400 $\left.\mathbf{~ M H z , ~} \mathbf{C D C l}_{3}\right): \delta 1.06$ (s, $3 \mathrm{H}), 1.13(\mathrm{~s}, 3 \mathrm{H}), 1.15$ (s, 3H), $1.28(\mathrm{~s}, 3 \mathrm{H}), 1.34(\mathrm{~s}, 3 \mathrm{H}), 1.53-1.74(\mathrm{~m}, 2 \mathrm{H}), 2.26(\mathrm{~s}, 3 \mathrm{H})$, 2.52 (s, 3H), 3.18 (brs, $1 \mathrm{H}), 6.64(\mathrm{~s}, 1 \mathrm{H}), 6.95(\mathrm{~s}, 1 \mathrm{H}), 7.22-7.27(\mathrm{~m}, 3 \mathrm{H}), 7.34(\mathrm{tt}, J=7.6$, $1.5 \mathrm{~Hz}, 1 \mathrm{H}), 7.43-7.48(\mathrm{~m}, 3 \mathrm{H}), 7.54(\mathrm{dd}, J=8.1,7.1 \mathrm{~Hz}, 1 \mathrm{H}), 7.69(\mathrm{dd}, J=8.1,6.9 \mathrm{~Hz}, 1 \mathrm{H})$, $7.88(\mathrm{dd}, J=8.1,1.0 \mathrm{~Hz}, 1 \mathrm{H}), 8.11(\mathrm{dd}, J=7.8,1.0 \mathrm{~Hz}, 1 \mathrm{H}), 8.21$ (dd, $J=6.7,1.2 \mathrm{~Hz}, 1 \mathrm{H}) .{ }^{13} \mathbf{C}$ NMR (125 MHz, $\left.\mathbf{C D C l}_{3}\right): \delta 20.3,21.1,22.1,31.5,31.8,32.3,32.4,54.6,71.0,78.7,125.5,126.1,127.7,128.27,128.29,129.7,129.9$, $130.8,131.8,132.0,133.27,133.28,133.9,135.35,135.40,135.75,135.84,136.2,137.6,139.0,142.8$, 146.7. IR 
(ATR-IR): 3493, 2969, 1570, 1428, 1366, 1194, 1112, 1022, 888, 851, 824, 779, 740, 700, 673, 653, 619, 568, 531, $517 \mathrm{~cm}^{-1}$. HRMS (ESI): $\mathrm{m} / z$ calculated for $\mathrm{C}_{34} \mathrm{H}_{38} \mathrm{NaO}_{2} \mathrm{Si}[\mathrm{M}+\mathrm{Na}]^{+}=529.2533$, found: 529.2531 .

4-((6,7-dimethoxy-1,2-diphenyl-1H-naphtho[1,8-bc]silin-1-yl)oxy)-2,4-dimethylpentan-2-ol (13)

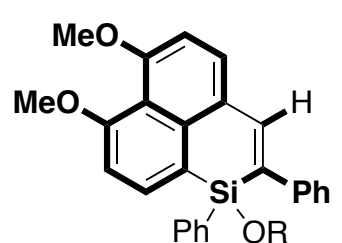

$\mathrm{Ph}^{\prime}$ 'OR

Following the procedure of the synthesis of $\mathbf{1 0}(0.20 \mathrm{mmol}$ scale $)$, the titled compound was obtained as yellow oil in 83\% yield (86.6 mg). ${ }^{1} \mathbf{H}$ NMR (400 $\left.\mathbf{~ M H z}, \mathbf{C D C l}_{3}\right): \delta 1.05(\mathrm{~s}, 3 \mathrm{H})$, $1.14(\mathrm{~s}, 3 \mathrm{H}), 1.22(\mathrm{~s}, 3 \mathrm{H}), 1.26(\mathrm{~s}, 3 \mathrm{H}), 1.60-1.68(\mathrm{~m}, 2 \mathrm{H}), 3.99(\mathrm{~s}, 3 \mathrm{H}), 4.02(\mathrm{~s}, 3 \mathrm{H}) 6.91$ $(\mathrm{d}, J=8.3 \mathrm{~Hz}, 1 \mathrm{H}), 6.99(\mathrm{~d}, J=8.1 \mathrm{~Hz}, 1 \mathrm{H}), 7.18(\mathrm{tt}, J=7.6,2.0 \mathrm{~Hz}, 1 \mathrm{H}), 7.27-7.32(\mathrm{~m}, 5 \mathrm{H})$, $7.53(\mathrm{dd}, J=8.2,1.2 \mathrm{~Hz}, 2 \mathrm{H}), 7.59(\mathrm{~d}, J=8.3 \mathrm{~Hz}, 1 \mathrm{H}), 7.63-7.65(\mathrm{~m}, 2 \mathrm{H}), 7.86(\mathrm{~d}, J=7.8 \mathrm{~Hz}$,

1H), 7.89 (s, 1H). ${ }^{13} \mathbf{C}$ NMR (125 MHz, $\mathbf{C D C l}_{3}$ ): $\delta 31.8,31.87,31.89,31.93,54.3,56.3,56.4,71.378 .7,105.7,107.0$, 116.8, 123.7, 126.2, 126.7, 127.1, 128.1, 128.6, 129.6, 134.4, 134.75, 134.79, 136.7, 137.4, 138.5, 142.4, 145.9, 159.0, 160.2. IR (ATR-IR): 3487, 2968, 1578, 1553, 1457, 1428, 1398, 1334, 1297, 1278, 1237, 1203, 1174, 1116, 986, 944, 902, 818, 755, 724, 697, 666, 605, 561, $514 \mathrm{~cm}^{-1}$. HRMS (ESI): $\mathrm{m} / z$ calculated for $\mathrm{C}_{33} \mathrm{H}_{36} \mathrm{NaO}_{4} \mathrm{Si}[\mathrm{M}+\mathrm{Na}]^{+}=$ 547.2275, found: 547.2276 .

2,4-dimethyl-4-((2-methyl-4,5-diphenyl-5H-1-thia-5-silaacenaphthylen-5-yl)oxy)pentan-2-ol (14)

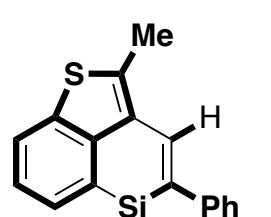

$\mathrm{Ph}^{\prime} \mathrm{O} \mathrm{OR}$

Following the procedure of the synthesis of $\mathbf{1 0}(0.11 \mathrm{mmol}$ scale), the titled compound was obtained as colorless oil in 58\% yield (31.0 mg). ${ }^{1} \mathbf{H}$ NMR (400 $\left.\mathbf{~ M H z}, \mathbf{C D C l}_{3}\right): \delta 1.15(\mathrm{~s}, 3 \mathrm{H})$, $1.179(\mathrm{~s}, 3 \mathrm{H}), 1.183(\mathrm{~s}, 3 \mathrm{H}), 1.27(\mathrm{~s}, 3 \mathrm{H}), 1.59-1.68(\mathrm{~m}, 2 \mathrm{H}), 2.77(\mathrm{~s}, 3 \mathrm{H}), 7.21(\mathrm{tt}, J=7.3,1.2 \mathrm{~Hz}$, $1 \mathrm{H}), 7.27-7.38(\mathrm{~m}, 6 \mathrm{H}), 7.49(\mathrm{dd}, J=7.8,1.5 \mathrm{~Hz}, 2 \mathrm{H}), 7.59-7.61(\mathrm{~m}, 2 \mathrm{H}), 7.71(\mathrm{dd}, J=7.1,1.0 \mathrm{~Hz}$, $1 \mathrm{H}), 7.837(\mathrm{~s}, 1 \mathrm{H}), 7.841(\mathrm{dd}, J=8.1,1.0 \mathrm{~Hz}, 1 \mathrm{H})$. The proton of hydroxy group was not detected. ${ }^{13}$ C NMR (125 MHz, $\mathbf{C D C l}_{3}$ ): $\delta$ 14.1, 31.85, 31.87 (2C overlapped), 32.2, 54.3, 71.2, 78.9, 123.8, 124.4, 127.0, 127.5, 128.2, 128.7, 129.5, 129.8, 130.2, 131.8, $134.5,134.9,136.1,136.2,136.7,142.2,143.0,143.2$. IR (ATR-IR): 3520, 2969, 1574, 1419, 1367, 1168, 1108, 1022, 988, 909, 827, 755, 697, 666, 633, 595, 560, $521 \mathrm{~cm}^{-1}$. HRMS (ESI): $\mathrm{m} / z$ calculated for $\mathrm{C}_{30} \mathrm{H}_{32} \mathrm{NaO}_{2} \mathrm{SSi}[\mathrm{M}+\mathrm{Na}]^{+}=507.1785$, found: 507.1766.

2,4-dimethyl-4-((1-phenyl-2-(4-(trifluoromethyl)phenyl)-1 $H$-[1,4]azasilino[3,2,1-hi]indol-1-yl)oxy)pentan2-ol (15)

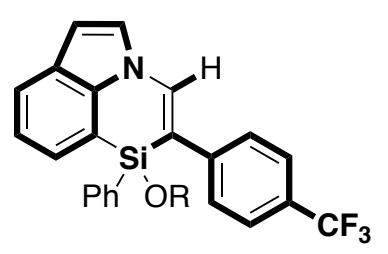

Following the procedure of the synthesis of $\mathbf{1 0}(0.10 \mathrm{mmol}$ scale $)$, the titled compound was obtained as slightly yellow oil in $47 \%$ yield $(24.6 \mathrm{mg}) .{ }^{1} \mathbf{H}$ NMR (500 $\left.\mathbf{~ M H z}, \mathbf{C D C l}_{3}\right)$ : $\delta 1.175(\mathrm{~s}, 3 \mathrm{H}), 1.184(\mathrm{~s}, 6 \mathrm{H}), 1.27(\mathrm{~s}, 3 \mathrm{H}), 1.67(\mathrm{~s}, 2 \mathrm{H}), 3.88$ (brs, $1 \mathrm{H}), 6.72(\mathrm{~d}, J=3.2$ $\mathrm{Hz}, 1 \mathrm{H}), 7.31-7.36(\mathrm{~m}, 5 \mathrm{H}), 7.52(\mathrm{~s}, 4 \mathrm{H}), 7.59-7.61(\mathrm{~m}, 2 \mathrm{H}), 7.64(\mathrm{dd}, J=7.0,0.9 \mathrm{~Hz}$, $1 \mathrm{H}), 7.78(\mathrm{dd}, J=7.8,0.9 \mathrm{~Hz}, 1 \mathrm{H}), 7.97(\mathrm{~s}, 1 \mathrm{H}) .{ }^{13} \mathbf{C} \mathbf{N M R}\left(\mathbf{1 2 5} \mathbf{~ M H z}, \mathbf{C D C l}_{3}\right): \delta 31.7$, 31.8, 32.1, 32.2, 54.4, 71.3, 79.1, 106.1, 117.7, 117.9, 123.1, 123.6, 124.5 (q, $J=272.5 \mathrm{~Hz}$ ), 125.8 (q, J=3.6 Hz), 127.4, $128.2,128.25(\mathrm{q}, J=32.7 \mathrm{~Hz}), 128.29,128.5,130.1,130.2,134.9,135.7,138.1,138.5,144.8 .{ }^{19}$ F NMR (470 MHz, CDCl $_{3}$ ): $\delta$-62.3. IR (ATR-IR): 3494, 2971, 1605, 1559, 1524, 1471, 1433, 1368, 1322, 1267, 1234, 1194, 1163, 1111 , 1066, 1015, 944, 907, 841, 792, 755, 723, 710, 699, 664, 605, $549 \mathrm{~cm}^{-1}$. HRMS (ESI): $\mathrm{m} / z$ calculated for $\mathrm{C}_{30} \mathrm{H}_{30} \mathrm{~F}_{3} \mathrm{NNaO}_{2} \mathrm{Si}[\mathrm{M}+\mathrm{Na}]^{+}=544.1890$, found: 544.1891 . 

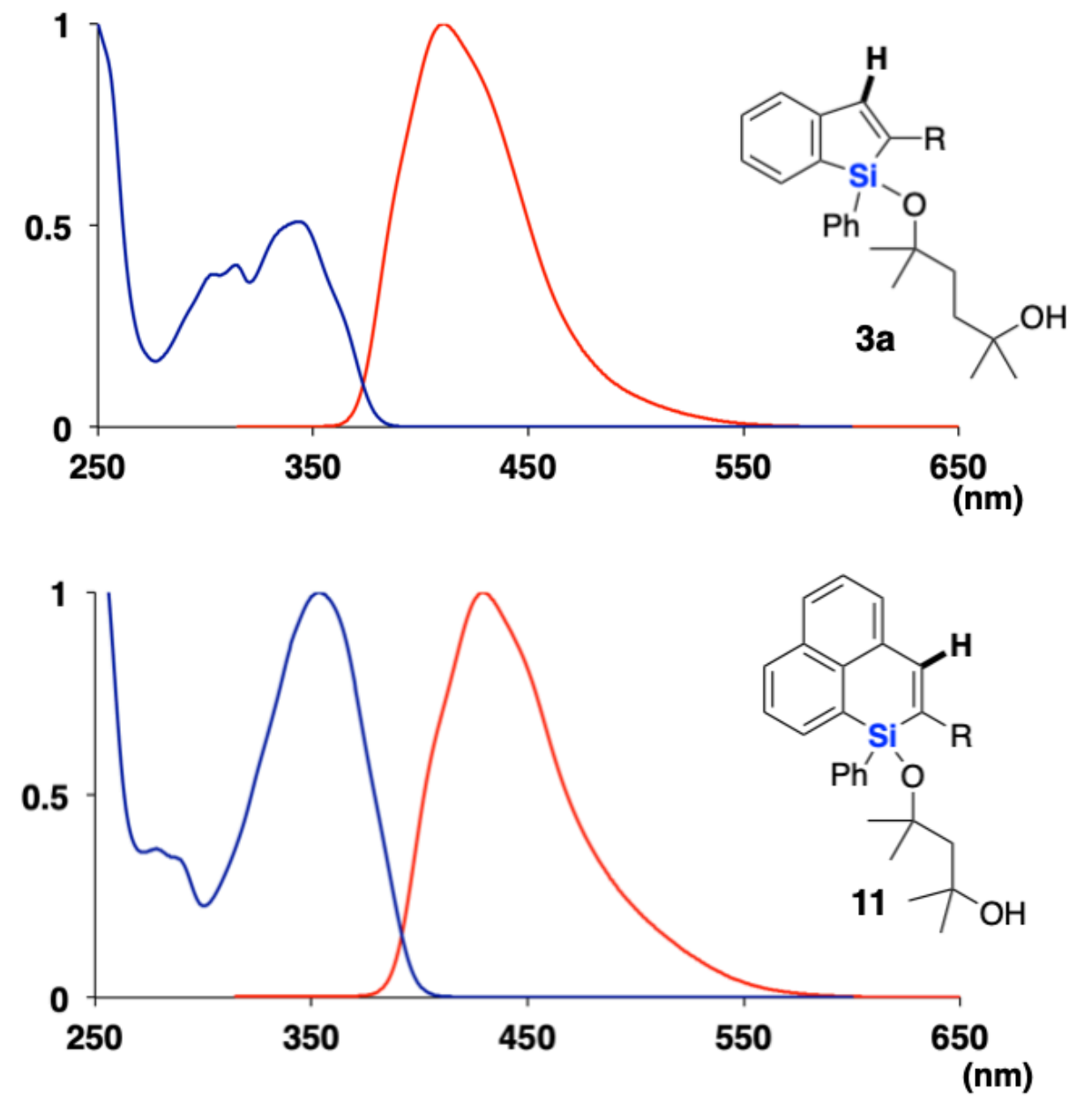

$\lambda_{\text {abs }}=343 \mathrm{~nm}$

$\lambda_{\mathrm{em}}=410 \mathrm{~nm}$

$\Phi_{\mathrm{FL}}=98 \%\left(\right.$ in $\left.\mathrm{CHCl}_{3}\right)$

— : absorption

: emission

$$
\begin{aligned}
\lambda_{\mathrm{abs}} & =354 \mathrm{~nm} \\
\lambda_{\mathrm{em}} & =430 \mathrm{~nm} \\
\Phi_{\mathrm{FL}} & =57 \%\left(\text { in } \mathrm{CHCl}_{3}\right)
\end{aligned}
$$

Figure S1.

${ }^{*} \mathrm{X}$-axis: wavelength, $\mathrm{Y}$-axis: normalized absorption and emission. 


\section{2-7. Computational Studies (Figure 1, Scheme 2, Table 3, and Scheme S1)}

All DFT calculations were carried with the Gaussian 16 program package ${ }^{19}$ with the help of the GRRM (The Global Reaction Route Mapping ${ }^{20,21}$ version 11) program utilizing the energies and energy derivatives from Gaussian 16 . The molecular structure optimizations were conducted at the B3LYP ${ }^{22}$ level in the gas phase using 6-31G $\mathrm{G}^{*}$ basis set for all the atoms (Figure 1). For Scheme 1 and Table 3c, the molecular structure optimizations were conducted at the M06-2 $\mathrm{X}^{23}$ level using 6-31+ $\mathrm{G}^{*}$ basis set for all the atoms and solvation was evaluated by the self-consistent reaction field (SCRF) method using the polarizable continuum model $(\mathrm{PCM}, \mathrm{SOLVENT}=\mathrm{THF}){ }^{24}$ The molecular structure optimizations in Table $3 \mathrm{~b}$ were conducted at the M06-2X level using 6-311++ $\mathrm{G}^{* *}$ basis set for all the atoms in combination with the self-consistent reaction field (SCRF) method using the polarizable continuum model (PCM, SOLVENT $=$ THF). The intrinsic reaction coordinate (IRC) method was used to track minimum energy paths from transition structures to the corresponding local minima. ${ }^{25}$ In this study, the Gibbs free energy was adopted as the basis for discussion. The vibrational frequencies were computed at the same level for all the calculations.

(19) Gaussian 16, Revision B.01, Frisch, M. J.; Trucks, G. W.; Schlegel, H. B.; Scuseria, G. E.; Robb, M. A.; Cheeseman, J. R.; Scalmani, G.; Barone, V.; Petersson, G. A.; Nakatsuji, H.; Li, X.; Caricato, M.; Marenich, A. V.; Bloino, J.; Janesko, B. G.; Gomperts, R.; Mennucci, B.; Hratchian, H. P.; Ortiz, J. V.; Izmaylov, A. F.; Sonnenberg, J. L.; Williams-Young, D.; Ding, F.; Lipparini, F.; Egidi, F.; Goings, J.; Peng, B.; Petrone, A.; Henderson, T.; Ranasinghe, D.; Zakrzewski, V. G.; Gao, J.; Rega, N.; Zheng, G.; Liang, W.; Hada, M.; Ehara, M.; Toyota, K.; Fukuda, R.; Hasegawa, J.; Ishida, M.; Nakajima, T.; Honda, Y.; Kitao, O.; Nakai, H.; Vreven, T.; Throssell, K.; Montgomery, J. A. Jr.; Peralta, J. E.; Ogliaro, F.; Bearpark, M. J.; Heyd, J. J.; Brothers, E. N.; Kudin, K. N.; Staroverov, V. N.; Keith, T. A.; Kobayashi, R.; Normand, J.; Raghavachari, K.; Rendell, A. P.; Burant, J. C.; Iyengar, S. S.; Tomasi, J.; Cossi, M.; Millam, J. M.; Klene, M.; Adamo, C.; Cammi, R.; Ochterski, J. W.; Martin, R. L.; Morokuma, K.; Farkas, O.; Foresman, J. B.; Fox, D. J. Gaussian, Inc., Wallingford CT, 2016.

(20) (a) Ohno, K.; Maeda, S. Chem. Phys. Lett. 2004, 384, 277. (b) Maeda, S.; Ohno, K. J. Phys. Chem. A 2005, 109, 5742. (c) Ohno, K.; Maeda, S. J. Phys. Chem. A 2006, 110, 8933. (d) Maeda, S.; Morokuma, K. J. Chem. Phys. 2010, $132,241102$.

(21) Maeda, S.; Ohno, K.; Morokuma, K. Phys. Chem. Chem. Phys. 2013, 15, 3683.

(22) (a) Becke, A. D. Phys. Rev. 1988, A38, 3098. (b) Becke, A. D. J. Chem. Phys. 1993, 98, 1372. (c) Becke, A. D. J. Chem. Phys. 1993, 98, 5648. (d) Lee, C.; Yang, W.; Parr, R. G. Phys. Rev. 1988, B37, 785.

(23) (a) Zhao, Y.; Truhlar, D. G. Theor. Chem. Acc. 2008, 120, 215. (b) Zhao, Y.; Truhlar, D. G. Acc. Chem. Res. 2008, $41,157$.

(24) (a) Miertuš, S.; Scrocco, E.; Tomasi, J. Chem. Phys. 1981, 55, 117. (b) Pascual-Ahuir, J. L.; Silla, E.; Tuñón, I.; J. Comp. Chem. 1994, 15, 1127. (c) Cancès, E.; Mennucci, B.; Tomasi, J. J. Chem. Phys. 1997, 107, 3032. (d) Cossi, M.; Barone, V.; Mennuci, B.; Tomasi, J. Chem. Phys. Lett. 1998, 286, 253. (e) Tomasi, J.; Mennucci, B.; Cancès, E. J. Mol. Struct. (THEOCHEM) 1999, 464, 21. (f) Tomasi, J.; Mennucci, B.; Cammi, R. Chem. Rev. 2005, 105, 2999.

(25) (a) Fukui, K. Acc. Chem. Res. 1981, 14, 363. (b) Ishida, K.; Morokuma, K.; Komornicki, A. J. Chem. Phys. 1977, 66, 2153. (c) Gonzalez, C.; Schlegel, H. B. J. Chem. Phys. 1989, 90, 2154. (d) Gonzalez, C.; Schlegel, H. B. J. Phys. Chem. 1990, 94, 5523. 


\section{2-7-1. DFT Calculations on Benzosilole Formation}

DFT calculations of the reaction pathway are summarized in Scheme S1. Coordination of the aryllithium to 1 a has a low activation barrier $(1.8 \mathrm{kcal} / \mathrm{mol})$, as expected, although the silicate formation is not highly favorable due to the steric congestion around the pentavalent silicon atom $\left(\Delta G_{\mathbf{C P 1}-\mathbf{C P 2}}=-0.1 \mathrm{kcal} / \mathrm{mol}\right)$. This highly reversible process is in good accordance with experimental finding (Table 1) that an excess of 1a increases the yield (Entries 4 and 5 ). The activation energy for hydride transfer from the silicon center to the carbon-carbon triple bond was computed to be $15.0 \mathrm{kcal} / \mathrm{mol}$ with a large energy gain of $-24.1 \mathrm{kcal} / \mathrm{mol}$ resulting from strain release as shown in the main text. The final silylative cyclization is also essentially barrierless, and rapid pseudo-rotation of CP6 to the more stable conformer CP7 followed by acidic workup gave the product.

\section{Scheme S1. DFT Calculations on Benzosilole Formation}
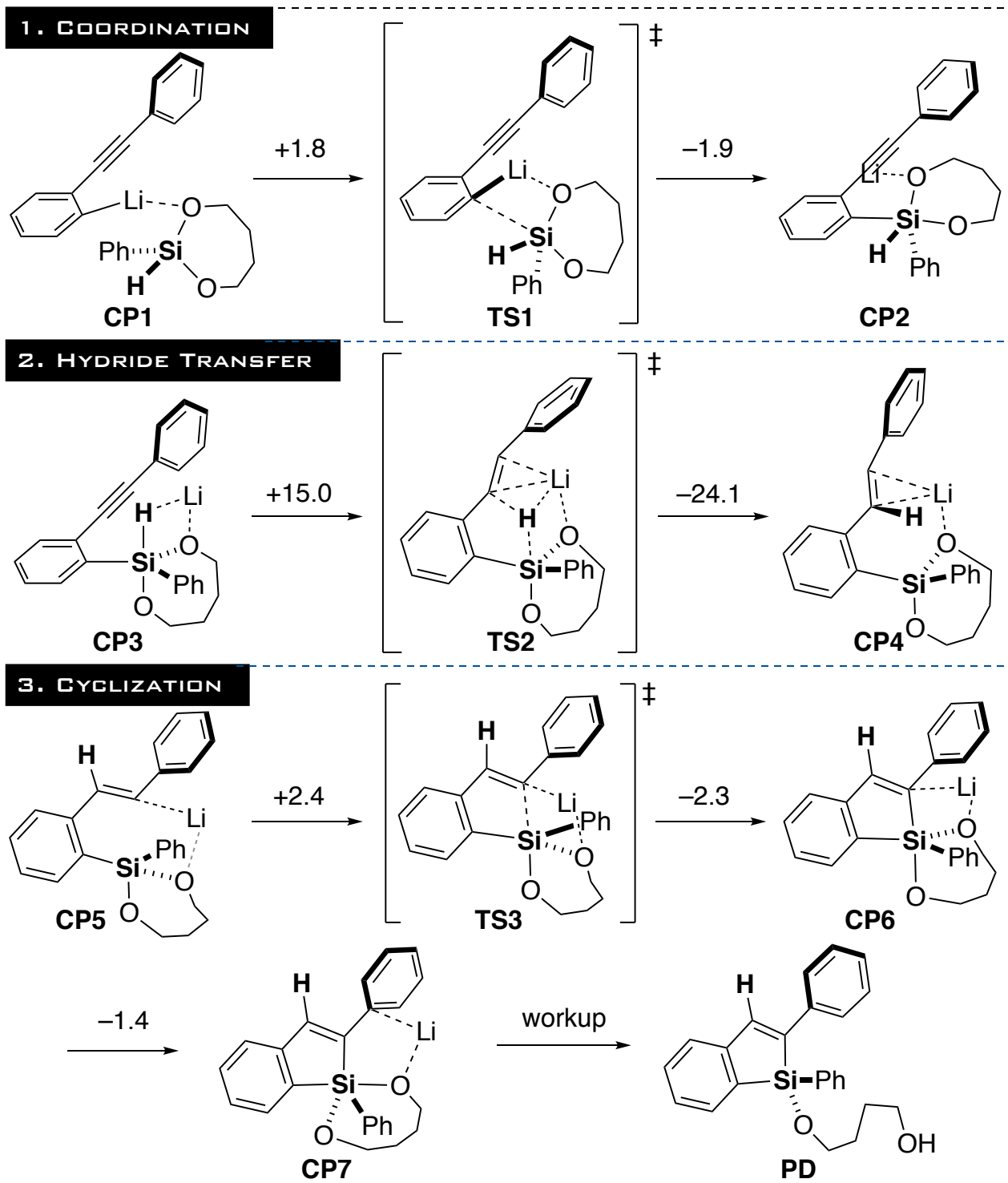

Calculated at the level of M06-2X/6-31+G* with PCM (THF). Numerical values are $\Delta G(\mathrm{kcal} / \mathrm{mol})$. Methyl groups on the ligand and one molecule of THF on a Li atom are omitted for clarity. 


\section{2-7-2. Cartesian Coordinates}

\section{- Figure 1}<smiles>CO[SiH2]OC</smiles>

CP1: Free Energy (RB3LYP): -568.366146685374 (hartree)

$\begin{array}{lccc}* * * * * * * * * * * * * * * * * * * * * * * * * * * * * * * * * * * * * * * * * * * * * * * * * * * * * * * * * * * * * * * * * * * * * * * * * * * * * * * * * * * * * * * \\ \mathrm{Si} & 1.214242299428 & 0.764781633683 & -0.069014911108 \\ \mathrm{O} & 0.842722147281 & -0.893353885921 & -0.077712313282 \\ \mathrm{C} & 1.834520706495 & -1.891005995339 & 0.226319769441 \\ \mathrm{H} & 2.252303652796 & -1.726618180839 & 1.226220010110 \\ \mathrm{H} & 1.348482440804 & -2.869102379558 & 0.201648191223 \\ \mathrm{H} & 2.637224543526 & -1.871019509199 & -0.519693732836 \\ \mathrm{H} & 2.216286105982 & 1.025566654020 & -1.122621124976 \\ \mathrm{H} & 1.712163805073 & 1.105814028370 & 1.288274415926 \\ \mathrm{Li} & -1.024014671262 & -1.292392785738 & -0.333003560660 \\ \mathrm{C} & -1.268460435435 & 1.914837346256 & 0.396951853672 \\ \mathrm{H} & -1.719761530226 & 2.814476790948 & -0.027941089925 \\ \mathrm{H} & -2.005669831233 & 1.104952736997 & 0.417599114897 \\ \mathrm{H} & -0.921261759189 & 2.130207209229 & 1.415081515292 \\ \mathrm{O} & -0.169793903314 & 1.546188476992 & -0.455643156387 \\ \mathrm{C} & -2.994546486904 & -1.532908875290 & -0.058397145313 \\ \mathrm{H} & -3.604097485597 & -0.903175497074 & -0.732408806775 \\ \mathrm{H} & -3.318640682887 & -2.571590747161 & -0.251354570571 \\ \mathrm{H} & -3.337795915337 & -1.302302020375 & 0.967149541272 \\ * * * * * * * * * * * * * * * * * * * * * * * * * * * * * * * * * * * * * * * * * * * * * * * * * * * * * * * * * * * * * * * * * * * * * * * * * * * * * * * * * * * * * * * * * *\end{array}$<smiles>CO[SiH](C)(C)OC</smiles>

CP2: Free Energy (RB3LYP): -568.393251286876 (hartree)

$\begin{array}{lccc}* * * * * * * * * * * * * * * * * * * * * * * * * * * * * * * * * * * * * * * * * * * * * * * * * * * * * * * * * * * * * * * * * * * * * * * * * * * * \\ \mathrm{Si} & 0.141830354740 & -0.647141520157 & 0.035409391467 \\ \mathrm{O} & -1.656603088938 & 0.180963492960 & -0.065883265840 \\ \mathrm{O} & 0.555481341216 & 1.097590158877 & -0.087580216589 \\ \mathrm{C} & -2.734344953320 & -0.715129311738 & -0.019577453458 \\ \mathrm{H} & -2.735051007784 & -1.292904116185 & 0.919420463932 \\ \mathrm{H} & -3.685432546283 & -0.168077170461 & -0.083163809463 \\ \mathrm{H} & -2.696083839410 & -1.431486579644 & -0.856786605500 \\ \mathrm{C} & 1.878562505540 & 1.619416767084 & -0.100982928995 \\ \mathrm{H} & 2.451632223205 & 1.238831532119 & -0.953576192582 \\ \mathrm{H} & 1.814179046164 & 2.711491722227 & -0.185547563873 \\ \mathrm{H} & 2.416190992427 & 1.374181502181 & 0.821494736349 \\ \mathrm{H} & -0.258203363882 & -1.391140955784 & -1.205212285997 \\ \mathrm{H} & -0.314207529671 & -1.198540832138 & 1.354817518302 \\ \mathrm{C} & 1.952123005677 & -1.335264542226 & 0.127520113572 \\ \mathrm{H} & 2.526795458491 & -1.015039989108 & 1.008174344236 \\ \mathrm{H} & 1.835448325524 & -2.427113129009 & 0.208155660942 \\ \mathrm{H} & 2.566336354260 & -1.150197263477 & -0.765126569982 \\ \mathrm{Li} & -1.101292022821 & 1.803435923708 & -0.176850809882\end{array}$


<smiles>CO[Si](C)([Hg])O[AlH]C</smiles>

TS1: Free Energy (RB3LYP): -568.390578428711 (hartree)

$\begin{array}{lccc}* * * * * * * * * * * * * * * * * * * * * * * * * * * * * * * * * * * * * * * * * * * * * * * * * * * * * * * * * * * * * * * * * * * * * * * * * * * * * * * * * * \\ \mathrm{Si} & 0.593270018803 & -0.752529026461 & 0.038856590285 \\ \mathrm{O} & -2.282768137488 & 0.417171017420 & -0.094333749155 \\ \mathrm{O} & 0.682592578594 & 0.941275006389 & -0.282278355426 \\ \mathrm{C} & -3.289760307058 & -0.486907524144 & 0.155025193112 \\ \mathrm{H} & -3.656098276446 & -0.438479118018 & 1.201022830492 \\ \mathrm{H} & -4.175111664283 & -0.329927012816 & -0.494341114508 \\ \mathrm{H} & -2.966293896719 & -1.537490128416 & -0.013353858792 \\ \mathrm{C} & 1.738074139138 & 1.741937786144 & 0.261602369445 \\ \mathrm{H} & 2.714740368228 & 1.390017176907 & -0.088236331763 \\ \mathrm{H} & 1.596101009911 & 2.771418151491 & -0.081632156086 \\ \mathrm{H} & 1.718583755457 & 1.728183579957 & 1.359211069917 \\ \mathrm{H} & -0.257473790689 & -1.329991658413 & -1.016638279105 \\ \mathrm{H} & 0.076425475200 & -0.966519838342 & 1.410731027897 \\ \mathrm{C} & 2.339670934723 & -1.459690804291 & -0.079544566179 \\ \mathrm{H} & 3.010150165145 & -1.087363338837 & 0.704433790372 \\ \mathrm{H} & 2.286706889076 & -2.551029290952 & 0.034085513872 \\ \mathrm{H} & 2.801257645541 & -1.255199329579 & -1.053714825364 \\ \mathrm{Li} & -1.125336907133 & 1.490148351958 & -0.490663149013 \\ * * * * * * * * * * * * * * * * * * * * * * * * * * * * * * * * * * * * * * * * * * * * * * * * * * * * * * * * * * * * * * * * * * * * * * * * * * * * * * * *\end{array}$<smiles>CO[AlH]OC</smiles>

CP3: Free Energy (RB3LYP): -568.399651857819 (hartree)

$\begin{array}{lccc}* * * * * * * * * * * * * * * * * * * * * * * * * * * * * * * * * * * * * * * * * * * * * * * * * * * * * * * * * * * * * * * * * * * * * * * * * * * * * * * * * * * * * * \\ \mathrm{Si} & 1.776329227741 & -0.881429066305 & 0.551507267515 \\ \mathrm{O} & -2.856497843548 & 0.517884221577 & -0.145909853013 \\ \mathrm{O} & 0.442671143507 & 0.102246398352 & 0.184257477662 \\ \mathrm{C} & -4.109220890460 & 1.044165113103 & -0.365807799545 \\ \mathrm{H} & -4.712906898793 & 1.112251613713 & 0.563311972192 \\ \mathrm{H} & -4.072065514539 & 2.071742964810 & -0.785822987929 \\ \mathrm{H} & -4.706997449827 & 0.440635868943 & -1.080438175944 \\ \mathrm{C} & 0.600730530171 & 1.512550562868 & -0.101810719687 \\ \mathrm{H} & 1.170770146189 & 1.649585405673 & -1.027068617073 \\ \mathrm{H} & -0.400663417859 & 1.933972932725 & -0.225076358422 \\ \mathrm{H} & 1.110935293000 & 2.010785742685 & 0.730502166137 \\ \mathrm{H} & 1.150178519798 & -2.171740414911 & 0.926840655965 \\ \mathrm{H} & 2.484654220538 & -0.282909796876 & 1.713839912147 \\ \mathrm{C} & 2.938089188031 & -1.064486663154 & -0.910271789575 \\ \mathrm{H} & 3.396623985410 & -0.109807431196 & -1.193990355711 \\ \mathrm{H} & 3.751842658419 & -1.755749838797 & -0.654407774747 \\ \mathrm{H} & 2.418163782376 & -1.468404345370 & -1.786834389333 \\ \mathrm{Li} & -1.441135009793 & -0.227630080365 & 0.137791331967 \\ * * * * * * * * * * * * * * * * * * * * * * * * * * * * * * * * * * * * * * * * * * * * * * * * * * * * * * * * * * * * * * * * * * * * * * * * * * * * * * * * *\end{array}$




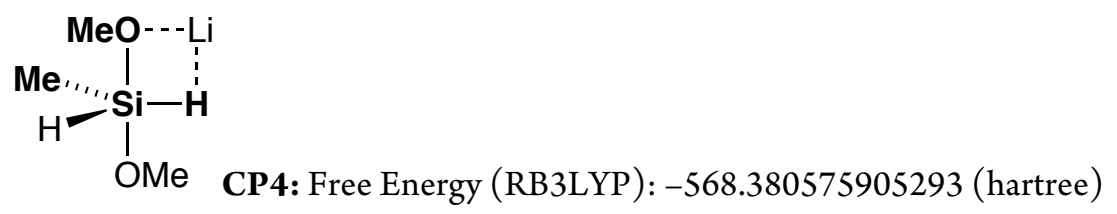

$\begin{array}{lccc}* * * * * * * * * * * * * * * * * * * * * * * * * * * * * * * * * * * * * * * * * * * * * * * * * * * * * * * * * * * * * * * * * * * * * * * * * * * * * * * * * * \\ \mathrm{Si} & -0.200890234545 & 0.075211581140 & 0.019272085714 \\ \mathrm{O} & -1.842588921258 & 0.630263690859 & -0.059994161829 \\ \mathrm{O} & 1.559867515901 & -0.919964004825 & 0.016674301511 \\ \mathrm{C} & -2.876832369319 & -0.116745884273 & 0.525247459667 \\ \mathrm{H} & -3.823615446160 & 0.422738877795 & 0.397365302596 \\ \mathrm{H} & -2.986261391767 & -1.110498675735 & 0.051889559758 \\ \mathrm{H} & -2.714530670715 & -0.273536844560 & 1.606347960321 \\ \mathrm{C} & 2.616773422716 & -0.469913446334 & 0.825739307718 \\ \mathrm{H} & 2.242450070099 & -0.132058862766 & 1.806146798510 \\ \mathrm{H} & 3.342244681086 & -1.277908147311 & 1.001176760486 \\ \mathrm{H} & 3.153005965107 & 0.370185215562 & 0.358311253884 \\ \mathrm{H} & -0.099430293041 & -0.035970082921 & 1.508686055132 \\ \mathrm{H} & -0.580885725337 & -1.212938811518 & -0.780016001208 \\ \mathrm{C} & 0.566782231288 & 1.523833180406 & -0.921295731356 \\ \mathrm{H} & 0.941001553379 & 1.198909576371 & -1.900445236717 \\ \mathrm{H} & -0.177209657097 & 2.311119971281 & -1.075643811973 \\ \mathrm{H} & 1.419741199465 & 1.945658228081 & -0.376685475788 \\ \mathrm{Li} & 0.868432661549 & -2.209391429188 & -0.859994503700 \\ * * * * * * * * * * * * * * * * * * * * * * * * * * * * * * * * * * * * * * * * * * * * * * * * * * * * * * * * * * * * * * * * * * * * * * * * * * * * * * * * * * * * * * * * * *\end{array}$<smiles>[3H][IH][Si](C)(OC)OC</smiles>

TS2: Free Free Energy (RB3LYP): -568.364982086629 (hartree)

$\begin{array}{lccc}* * * * * * * * * * * * * * * * * * * * * * * * * * * * * * * * * * * * * * * * * * * * * * * * * * * * * * * * * * * * * * * * * * * * * * * * * * * * * * * * * * * * * * * * * * \\ \mathrm{Si} & -0.096471940767 & 0.291090842343 & 0.541653007734 \\ \mathrm{O} & -1.422114563876 & 0.309511404065 & -0.454625799833 \\ \mathrm{O} & 1.152397122449 & -0.871242154179 & 0.140560083755 \\ \mathrm{C} & -2.632031545704 & -0.436118072717 & -0.427606845978 \\ \mathrm{H} & -3.318002432202 & 0.046322535118 & -1.132760510315 \\ \mathrm{H} & -2.459504074726 & -1.475323013456 & -0.725131616239 \\ \mathrm{H} & -3.077535928391 & -0.441219178010 & 0.572828495330 \\ \mathrm{C} & 2.272440707907 & -0.557816316225 & -0.697272968319 \\ \mathrm{H} & 2.895995730664 & 0.221140214548 & -0.247114948105 \\ \mathrm{H} & 2.874527192834 & -1.465784465474 & -0.811904738743 \\ \mathrm{H} & 1.941768125164 & -0.227100978172 & -1.687802867057 \\ \mathrm{H} & -0.310052307532 & 0.359436600184 & 2.001707639948 \\ \mathrm{H} & -0.987104012174 & -1.853345495559 & 1.199780302883 \\ \mathrm{C} & 0.699567704959 & 1.942868052822 & 0.066143148847 \\ \mathrm{H} & 0.895133398168 & 2.048451888550 & -1.007956293202 \\ \mathrm{H} & 0.001122939160 & 2.742419124970 & 0.344808698748 \\ \mathrm{H} & 1.636658324008 & 2.126575604632 & 0.608528394957 \\ \mathrm{Li} & 0.455097560056 & -2.452343593438 & 0.720174815588 \\ * * * * * * * * * * * * * * * * * * * * * * * * * * * * * * * * * * * * * * * * * * * * * * * * * * * * * * * * * * * * * * * * * * * * * * * * * * * * * * * * * * * * * * * * * *\end{array}$


<smiles>CO[SiH](C)O[SiH]</smiles>

CP5: Free Energy (RB3LYP): -568.378731618227 (hartree)

$\begin{array}{lccc}* * * * * * * * * * * * * * * * * * * * * * * * * * * * * * * * * * * * * * * * * * * * * * * * * * * * * * * * * * * * * * * * * * * * * * * * * * * * * * * * * * * * \\ \mathrm{Si} & -0.119200473321 & 0.429505474251 & 0.529357561174 \\ \mathrm{O} & -1.340310544847 & 0.494300333679 & -0.582199859183 \\ \mathrm{O} & 0.922566847087 & -0.878042854128 & 0.199161181915 \\ \mathrm{C} & -2.389697799560 & -0.465924383070 & -0.721544792657 \\ \mathrm{H} & -3.163497185875 & -0.020789240740 & -1.352286881255 \\ \mathrm{H} & -2.019225380754 & -1.379903704026 & -1.200924780828 \\ \mathrm{H} & -2.826974254609 & -0.722784574797 & 0.251748275703 \\ \mathrm{C} & 1.693722041178 & -0.939623601092 & -1.021564464309 \\ \mathrm{H} & 2.460662649911 & -0.158130592662 & -1.023607998852 \\ \mathrm{H} & 2.179778238070 & -1.917856586664 & -1.064043092771 \\ \mathrm{H} & 1.040575662804 & -0.823933232791 & -1.892381940004 \\ \mathrm{H} & -0.627915095249 & 0.054937222192 & 1.869692645108 \\ \mathrm{H} & 1.413504331269 & -3.786914552466 & 2.123457963769 \\ \mathrm{C} & 0.787513312145 & 2.055770895775 & 0.449806531858 \\ \mathrm{H} & 1.097723820389 & 2.294738926597 & -0.574047468194 \\ \mathrm{H} & 0.132108407733 & 2.865874899135 & 0.790893077685 \\ \mathrm{H} & 1.677842757720 & 2.047667813787 & 1.089493546547 \\ \mathrm{Li} & 1.126275169771 & -2.377123953779 & 1.342115490823 \\ * * * * * * * * * * * * * * * * * * * * * * * * * * * * * * * * * * * * * * * * * * * * * * * * * * * * * * * * * * * * * * * * * * * * * * * * * * * * * * * * * * *\end{array}$

- Scheme S1 (Me and THF are omitted for clarity)<smiles>C(#C[SiH]1OCCCO[Si]1(c1ccccc1)c1ccccc1)c1ccccc1</smiles>

CP1: Free Energy (RM062X): -1764.443649524466 (hartree)

$\begin{array}{lccc}* * * * * * * * * * * * * * * * * * * * * * * * * * * * * * * * * * * * * * * * * * * * * * * * * * * * * * * * * * * * * * * * * * * * * * * * * * * * * * * * * * * \\ \mathrm{C} & 0.303728226325 & -3.623567448063 & -0.077958469545 \\ \mathrm{C} & 0.799644918957 & -2.316504476184 & 0.099515925374 \\ \mathrm{C} & 1.543476388217 & -2.155883156077 & 1.299837072922 \\ \mathrm{C} & 1.767627271197 & -3.175975205112 & 2.241652539210 \\ \mathrm{C} & 1.240779616341 & -4.441633412154 & 2.004056462198 \\ \mathrm{C} & 0.507010269387 & -4.664523787980 & 0.833652431666 \\ \mathrm{H} & -0.283598984477 & -3.849503243597 & -0.972691818010 \\ \mathrm{H} & 2.342205695809 & -2.976645758994 & 3.144125086037 \\ \mathrm{H} & 1.398339496624 & -5.244487593902 & 2.719206840916 \\ \mathrm{H} & 0.092953164330 & -5.652721964593 & 0.640153931207 \\ \mathrm{C} & 2.072269530596 & -0.829877804189 & 1.527036395889 \\ \mathrm{C} & 2.440687904692 & 0.330141237136 & 1.575901883376 \\ \mathrm{C} & 2.819804857018 & 1.714234792485 & 1.633769816712 \\ \mathrm{C} & 2.222565010269 & 2.574440684130 & 2.569214800923 \\ \mathrm{C} & 3.755770565029 & 2.232838615679 & 0.725148544554 \\ \mathrm{C} & 2.553040013686 & 3.926955318107 & 2.588728806323 \\ \mathrm{H} & 1.497035921056 & 2.173227276207 & 3.270988543626 \\ \mathrm{C} & 4.080031257509 & 3.586542172106 & 0.74781115762\end{array}$

S-30 


\begin{tabular}{|c|c|}
\hline $\mathrm{H}$ & 4.219408188422 \\
\hline C & 3.479167766622 \\
\hline $\mathrm{H}$ & 2.084667140866 \\
\hline $\mathrm{H}$ & 4.803194348936 \\
\hline $\mathrm{H}$ & 3.733309406409 \\
\hline $\mathrm{Si}$ & -1.930666138452 \\
\hline $\mathrm{H}$ & -1.752496993824 \\
\hline $\mathrm{C}$ & -2.042058756960 \\
\hline C & -2.909369723339 \\
\hline C & -1.266472646775 \\
\hline $\mathrm{C}$ & -3.005674299727 \\
\hline $\mathrm{H}$ & -3.517004125184 \\
\hline C & -1.359270524963 \\
\hline $\mathrm{H}$ & -0.569323955160 \\
\hline C & -2.230792836285 \\
\hline $\mathrm{H}$ & -3.679896671344 \\
\hline $\mathrm{H}$ & -0.745872214652 \\
\hline $\mathrm{H}$ & -2.301221043432 \\
\hline $\mathrm{C}$ & -0.673623735424 \\
\hline C & -3.239347639127 \\
\hline C & -4.043434747191 \\
\hline $\mathrm{H}$ & -3.820949409249 \\
\hline $\mathrm{H}$ & -3.190516844283 \\
\hline $\mathrm{O}$ & -0.644050633198 \\
\hline $\mathrm{O}$ & -3.306497174766 \\
\hline $\mathrm{Li}$ & 1.006648719900 \\
\hline $\mathrm{C}$ & 1.419471234192 \\
\hline $\mathrm{O}$ & 2.054590904408 \\
\hline $\mathrm{C}$ & 3.422473387899 \\
\hline $\mathrm{C}$ & 3.717095910384 \\
\hline C & 2.340811306560 \\
\hline $\mathrm{H}$ & 0.421683801882 \\
\hline $\mathrm{H}$ & 1.328532013707 \\
\hline $\mathrm{H}$ & 4.054156978615 \\
\hline $\mathrm{H}$ & 3.496808190714 \\
\hline $\mathrm{H}$ & 4.049405447208 \\
\hline $\mathrm{H}$ & 4.484955373948 \\
\hline $\mathrm{H}$ & 2.237111087230 \\
\hline $\mathrm{H}$ & 2.126348163657 \\
\hline $\mathrm{C}$ & -1.826752836683 \\
\hline $\mathrm{H}$ & -1.823933504316 \\
\hline $\mathrm{H}$ & -1.542266119639 \\
\hline $\mathrm{C}$ & 0.645395714198 \\
\hline $\mathrm{H}$ & 0.690302869655 \\
\hline $\mathrm{H}$ & 1.497107173364 \\
\hline $\mathrm{H}$ & 0.746854467595 \\
\hline $\mathrm{C}$ & -0.733596594568 \\
\hline $\mathrm{H}$ & 0.178170120039 \\
\hline $\mathrm{H}$ & -1.596069373944 \\
\hline $\mathrm{H}$ & -0.808470389771 \\
\hline C & -4.393466553724 \\
\hline $\mathrm{H}$ & -4.901768869443 \\
\hline $\mathrm{H}$ & -3.511238128186 \\
\hline $\mathrm{H}$ & -5.063665849516 \\
\hline $\mathrm{C}$ & -5.327193092575 \\
\hline $\mathrm{H}$ & -5.935969699009 \\
\hline
\end{tabular}

1.566831593854

4.437218989886

4.584572023367

3.978550478277

5.492684673279

$-0.543002018189$

$-1.796160448998$

$-0.788789760968$

$-1.750680652736$

$-0.024490430672$

$-1.942001199435$

$-2.365210019809$

$-0.208412772533$

0.712848138922

$-1.167558131727$

$-2.694425638165$

0.387704007803

$-1.316008801681$

1.815318529664

2.493668775713

1.283870711383

3.390828610900

2.453793612358

0.505864024452

0.082018230754

$-0.443465089102$

$-1.439492810562$

$-0.475970142231$

$-0.865635231275$

$-1.994887345737$

$-2.651716505611$

$-1.649158580862$

$-0.993584915726$

0.015686880054

$-1.210619999015$

$-1.589462705149$

$-2.674807947486$

$-3.250268098763$

$-3.278051656931$

2.679327078144

2.578413994522

3.719385977702

2.477477040697

2.624540925620

1.859782127212

3.452134271213

1.619455159329

1.115981871851

1.008773832170

2.585478118922

1.402535751481

0.493144619020

1.543586360670

2.254815398081

1.159766840327

2.064411212111
0.002993373360

1.677497245292

3.314881589524

0.038924209691

1.693205074815

$-0.711064205864$

$-1.460809762899$

1.134167970674

1.670700985768

2.016782761985

3.049007818747

1.007770056272

3.395908829382

1.621308508723

3.913757470941

3.448068130238

4.066306138533

4.987556238038

$-1.599082976507$

$-1.617770628741$

$-1.123446752879$

$-1.371385187985$

$-2.712792108783$

$-0.974410916729$

$-1.381299810434$

$-0.755126996420$

$-3.238319536511$

$-2.368950939424$

$-2.116678632327$

$-3.098859128567$

$-3.239574261682$

$-2.838621298935$

$-4.236119176292$

$-2.253942394963$

$-1.076987351991$

$-4.060614038283$

$-2.723260610070$

$-4.147723025135$

$-2.367361945124$

$-1.049087545954$

0.045392916555

$-1.248417094511$

$-1.200160210367$

$-0.114326599655$

$-1.506890132565$

$-1.687479987027$

$-3.111085213044$

$-3.448089694886$

$-3.395984188212$

$-3.622618446233$

0.360889887190

0.697848206850

0.991477022056

0.514310145591

$-1.940986118312$

$-1.844229191449$ 


$\begin{array}{llcc}\mathrm{H} & -5.086919645591 & 1.010046306445 & -2.998353563085 \\ \mathrm{H} & -5.914882735631 & 0.304381819715 & -1.593022033911 \\ * * * * * * * * * * * * * * * * * * * * * * * * * * * * * * * * * * * * * * * * * * * * * * * * * * * * * * * * * * * * * * * * * * * * * * * * * * * * * * * * * * * * * * * * * * * * *\end{array}$<smiles>[CH2+]C(C#Cc1ccccc1)[Si]1(c2ccccc2)OCCCO1</smiles>

TS1: Free Energy (RM062X): -1764.440705740086 (hartree)

\begin{tabular}{|c|c|c|c|}
\hline $\mathrm{C}$ & -0.016636671883 & -3.413216093025 & -0.126750209599 \\
\hline $\mathrm{C}$ & 0.428990256434 & -2.100810666922 & 0.115368492136 \\
\hline $\mathrm{C}$ & 1.582802270874 & -2.042202177504 & 0.945736527464 \\
\hline $\mathrm{C}$ & 2.248264817481 & -3.171154584534 & 1.461414022602 \\
\hline $\mathrm{C}$ & 1.756537002025 & -4.438464170758 & 1.171974065247 \\
\hline $\mathrm{C}$ & 0.610148025685 & -4.555722028315 & 0.377992612709 \\
\hline $\mathrm{H}$ & -0.902391052697 & -3.564274760677 & -0.750345967709 \\
\hline $\mathrm{H}$ & 3.134358904606 & -3.048614761016 & 2.080749535982 \\
\hline $\mathrm{H}$ & 2.253692717439 & -5.322558094354 & 1.561104710327 \\
\hline $\mathrm{H}$ & 0.213568842728 & -5.543465550762 & 0.150520473007 \\
\hline $\mathrm{C}$ & 2.096481701798 & -0.725113219388 & 1.241989744969 \\
\hline $\mathrm{C}$ & 2.427526866714 & 0.438589962739 & 1.381995812496 \\
\hline $\mathrm{C}$ & 2.822803111707 & 1.808759492470 & 1.550313133546 \\
\hline $\mathrm{C}$ & 1.857323597632 & 2.794869128908 & 1.811361807443 \\
\hline $\mathrm{C}$ & 4.173027076397 & 2.178968039860 & 1.445321596873 \\
\hline $\mathrm{C}$ & 2.237822273656 & 4.125279240047 & 1.960714994322 \\
\hline $\mathrm{H}$ & 0.811962800209 & 2.508730376390 & 1.892270146808 \\
\hline $\mathrm{C}$ & 4.546714201217 & 3.511402073616 & 1.598721146132 \\
\hline $\mathrm{H}$ & 4.919035165950 & 1.416073169477 & 1.243208093122 \\
\hline $\mathrm{C}$ & 3.582039007313 & 4.487013534260 & 1.855108131606 \\
\hline $\mathrm{H}$ & 1.484234062370 & 4.880730388047 & 2.161688466789 \\
\hline $\mathrm{H}$ & 5.593242239721 & 3.788779140568 & 1.516722014954 \\
\hline $\mathrm{H}$ & 3.876585353415 & 5.525294171895 & 1.973290615928 \\
\hline $\mathrm{Si}$ & -1.895381262677 & -0.516054593788 & -0.575906682112 \\
\hline $\mathrm{H}$ & -1.792506812686 & -1.680006651966 & -1.471459167065 \\
\hline $\mathrm{C}$ & -2.137963854959 & -0.851828631105 & 1.247041342264 \\
\hline $\mathrm{C}$ & -3.240345550768 & -1.619129934773 & 1.645641089504 \\
\hline $\mathrm{C}$ & -1.297704248872 & -0.334330897279 & 2.241750427805 \\
\hline $\mathrm{C}$ & -3.490905241221 & -1.875533920398 & 2.996117685730 \\
\hline $\mathrm{H}$ & -3.920761343273 & -2.021436330337 & 0.896257012470 \\
\hline $\mathrm{C}$ & -1.550565087643 & -0.568039238291 & 3.591315624020 \\
\hline $\mathrm{H}$ & -0.424488507217 & 0.247804965856 & 1.954839805715 \\
\hline $\mathrm{C}$ & -2.648194027016 & -1.345196897328 & 3.971711000340 \\
\hline $\mathrm{H}$ & -4.345275849471 & -2.481607920055 & 3.284581265338 \\
\hline $\mathrm{H}$ & -0.888011393749 & -0.154805875926 & 4.347136077300 \\
\hline $\mathrm{H}$ & -2.842331109714 & -1.536828543800 & 5.023276653884 \\
\hline $\mathrm{C}$ & -0.781566525437 & 1.731242336918 & -1.846055096642 \\
\hline $\mathrm{C}$ & -3.326885190242 & 2.443696497845 & -1.773633454794 \\
\hline $\mathrm{C}$ & -3.965960594543 & 1.372798989591 & -0.879636679133 \\
\hline $\mathrm{H}$ & -3.904213275338 & 3.369294202190 & -1.651437282154 \\
\hline
\end{tabular}




\begin{tabular}{|c|c|c|c|}
\hline $\mathrm{H}$ & -3.458013311240 & 2.133954270319 & -2.818467557379 \\
\hline $\mathrm{O}$ & -0.731063896159 & 0.690654183966 & -0.829141998330 \\
\hline $\mathrm{O}$ & -3.337629508742 & 0.116793642315 & -1.145083236088 \\
\hline $\mathrm{Li}$ & 0.909629957706 & -0.252094965841 & -0.719954439700 \\
\hline $\mathrm{C}$ & 1.618093885359 & -1.822661036801 & -2.950784809071 \\
\hline $\mathrm{O}$ & 2.053326647261 & -0.660199381551 & -2.218864860234 \\
\hline $\mathrm{C}$ & 3.484450721672 & -0.721402680977 & -2.019784445895 \\
\hline $\mathrm{C}$ & 3.943795234454 & -2.045976940055 & -2.637919701454 \\
\hline $\mathrm{C}$ & 2.659259041056 & -2.881788441140 & -2.628557316547 \\
\hline $\mathrm{H}$ & 0.608794187000 & -2.069328180012 & -2.610720754831 \\
\hline $\mathrm{H}$ & 1.602651688681 & -1.581035969128 & -4.021735892495 \\
\hline $\mathrm{H}$ & 3.936756887475 & 0.155715516923 & -2.491557466468 \\
\hline $\mathrm{H}$ & 3.671498816245 & -0.686386195272 & -0.941853712657 \\
\hline $\mathrm{H}$ & 4.287440882855 & -1.892380114414 & -3.666075016783 \\
\hline $\mathrm{H}$ & 4.757512767530 & -2.499224669466 & -2.067424644862 \\
\hline $\mathrm{H}$ & 2.670326182327 & -3.696287370015 & -3.356906578850 \\
\hline $\mathrm{H}$ & 2.466230508799 & -3.293339654465 & -1.630561748502 \\
\hline $\mathrm{C}$ & -1.855580129233 & 2.780412931359 & -1.514152766392 \\
\hline $\mathrm{H}$ & -1.706422330272 & 3.087141140674 & -0.470837031417 \\
\hline $\mathrm{H}$ & -1.618602736745 & 3.662048546009 & -2.122805540711 \\
\hline $\mathrm{C}$ & 0.584863163705 & 2.416288339803 & -1.784232771460 \\
\hline $\mathrm{H}$ & 0.747230792022 & 2.859873568536 & -0.795915968539 \\
\hline $\mathrm{H}$ & 1.391591806940 & 1.703866395788 & -1.991218311235 \\
\hline $\mathrm{H}$ & 0.645260450833 & 3.210193748337 & -2.534907308154 \\
\hline $\mathrm{C}$ & -0.979293703924 & 1.090125380335 & -3.218655920511 \\
\hline $\mathrm{H}$ & -0.137354327142 & 0.425111765381 & -3.441358166405 \\
\hline $\mathrm{H}$ & -1.906493814908 & 0.511872162040 & -3.264153290664 \\
\hline $\mathrm{H}$ & -1.020283108064 & 1.863751187057 & -3.993249127910 \\
\hline $\mathrm{C}$ & -3.881783743492 & 1.759930202721 & 0.599269891303 \\
\hline $\mathrm{H}$ & -4.378089994902 & 1.008098534050 & 1.220788900019 \\
\hline $\mathrm{H}$ & -2.848083195280 & 1.847286510901 & 0.947455627848 \\
\hline $\mathrm{H}$ & -4.376092179653 & 2.725408983959 & 0.754004278402 \\
\hline $\mathrm{C}$ & -5.431354890448 & 1.194324147085 & -1.273325504318 \\
\hline $\mathrm{H}$ & -5.995624846321 & 2.119020085317 & -1.113829115346 \\
\hline $\mathrm{H}$ & -5.506998364387 & 0.914280509779 & -2.329131026479 \\
\hline $\mathrm{H}$ & -5.883882236980 & 0.400083678094 & -0.670692257461 \\
\hline
\end{tabular}<smiles>[O-][Si](OCCCO)(c1ccccc1)c1ccccc1C#Cc1ccccc1</smiles>

CP2: Free Energy (RM062X): -1764.443759616209 (hartree)

$\begin{array}{lccc}* * * * * * * * * * * * * * * * * * * * * * * * * * * * * * * * * * * * * * * * * * * * * * * * * * * * * * * * * * * * * * * * * * * * * * * * * * * * * * * * * * * * * * * * * * \\ \mathrm{C} & -0.213308302168 & -3.290198680664 & -0.315831764768 \\ \mathrm{C} & 0.114240078091 & -1.953251159008 & -0.016072569427 \\ \mathrm{C} & 1.322197242942 & -1.807596701361 & 0.722188627376 \\ \mathrm{C} & 2.114057425180 & -2.902951505147 & 1.125555129100 \\ \mathrm{C} & 1.719096227456 & -4.197069646397 & 0.822028643568 \\ \mathrm{C} & 0.542730834922 & -4.387841632588 & 0.091965491881 \\ \mathrm{H} & -1.124626724594 & -3.484800745566 & -0.881175739591\end{array}$




\begin{tabular}{|c|c|}
\hline $\mathrm{H}$ & 3.027924861900 \\
\hline $\mathrm{H}$ & 2.318597548299 \\
\hline $\mathrm{H}$ & 0.219661639298 \\
\hline $\mathrm{C}$ & 1.796803952238 \\
\hline $\mathrm{C}$ & 2.142605458340 \\
\hline $\mathrm{C}$ & 2.431558406798 \\
\hline C & 1.362216111166 \\
\hline $\mathrm{C}$ & 3.750715438620 \\
\hline $\mathrm{C}$ & 1.612538372369 \\
\hline $\mathrm{H}$ & 0.344980827122 \\
\hline $\mathrm{C}$ & 3.992493456865 \\
\hline $\mathrm{H}$ & 4.574946645862 \\
\hline C & 2.927242145148 \\
\hline $\mathrm{H}$ & 0.782060059919 \\
\hline $\mathrm{H}$ & 5.014727091524 \\
\hline $\mathrm{H}$ & 3.121188390159 \\
\hline $\mathrm{Si}$ & -1.525868132509 \\
\hline $\mathrm{H}$ & -1.919047701375 \\
\hline $\mathrm{C}$ & -2.086899577093 \\
\hline $\mathrm{C}$ & -3.369105026383 \\
\hline C & -1.325112636755 \\
\hline $\mathrm{C}$ & -3.868294358101 \\
\hline $\mathrm{H}$ & -3.999354353279 \\
\hline $\mathrm{C}$ & -1.825864062207 \\
\hline $\mathrm{H}$ & -0.324643222300 \\
\hline $\mathrm{C}$ & -3.100203150042 \\
\hline $\mathrm{H}$ & -4.860802952915 \\
\hline $\mathrm{H}$ & -1.219782317191 \\
\hline $\mathrm{H}$ & -3.488929558531 \\
\hline $\mathrm{C}$ & -0.587363318956 \\
\hline $\mathrm{C}$ & -2.976086961202 \\
\hline $\mathrm{C}$ & -3.450708901981 \\
\hline $\mathrm{H}$ & -3.444788971669 \\
\hline $\mathrm{H}$ & -3.346759090255 \\
\hline $\mathrm{O}$ & -0.411931186803 \\
\hline $\mathrm{O}$ & -2.970150199006 \\
\hline $\mathrm{Li}$ & 1.248345087213 \\
\hline $\mathrm{C}$ & 1.806240375653 \\
\hline $\mathrm{O}$ & 2.394743519235 \\
\hline $\mathrm{C}$ & 3.733895101732 \\
\hline $\mathrm{C}$ & 4.023324000807 \\
\hline $\mathrm{C}$ & 2.620374842640 \\
\hline $\mathrm{H}$ & 0.748341374176 \\
\hline $\mathrm{H}$ & 1.895859135805 \\
\hline $\mathrm{H}$ & 4.411070483943 \\
\hline $\mathrm{H}$ & 3.741461362281 \\
\hline $\mathrm{H}$ & 4.479610944687 \\
\hline $\mathrm{H}$ & 4.693277084882 \\
\hline $\mathrm{H}$ & 2.560240591362 \\
\hline $\mathrm{H}$ & 2.269848009854 \\
\hline $\mathrm{C}$ & -1.464074004124 \\
\hline $\mathrm{H}$ & -1.084381756524 \\
\hline $\mathrm{H}$ & -1.269432364323 \\
\hline $\mathrm{C}$ & 0.822115820550 \\
\hline $\mathrm{H}$ & 1.236894601230 \\
\hline $\mathrm{H}$ & 1.501416203465 \\
\hline
\end{tabular}

$-2.720226022003$

$-5.043569187746$

$-5.394185362785$

$-0.489814512897$

0.664206331030

2.051961195080

2.953832602171

2.523652183403

4.309159350223

2.575424611648

3.881369003203

1.823848571428

4.775230562204

5.001935215381

4.243024385374

5.833148360667

$-0.644585812838$

$-1.651753802386$

$-0.982584546317$

$-1.523673117918$

$-0.729039819488$

$-1.806914885187$

$-1.708819939785$

$-0.972996288262$

$-0.311946731830$

$-1.520867285177$

$-2.236407769587$

$-0.742080260058$

$-1.722771940785$

1.634826095044

2.591496090725

1.522704321219

3.551733547108

2.316272248298

0.608053360019

0.266761021317

$-0.170160090397$

$-2.011862054776$

$-0.993770744131$

$-1.378469614025$

$-2.676843494700$

$-3.262734827765$

$-2.098806369369$

$-1.695275828618$

$-0.560609737714$

$-1.528692178034$

$-2.465138918946$

$-3.331080202057$

$-3.995295377487$

$-3.725032664438$

2.800395323841

3.116655505442

3.638811420311

2.192611414480

2.600694998930

1.422143622578
1.685511630723

1.143268087242

$-0.162781852351$

1.060295955723

1.227296561711

1.443749848641

1.571491670126

1.511508690347

1.759647403185

1.517524468397

1.703778973515

1.412423675990

1.825878680832

1.855952438796

1.756603304850

1.973699963313

$-0.430769125150$

$-1.457419486818$

1.371327803616

1.558646549315

2.521054609707

2.832115321610

0.690956864959

3.800493402480

2.425003322248

3.960864244111

2.943593735797

4.672999108145

4.955267492971

$-1.926359222870$

$-1.388621743184$

$-0.395218266975$

$-1.133516999462$

$-2.384925789794$

$-0.918238843986$

$-0.832006096951$

$-1.056255797824$

$-3.136722442998$

$-2.298784819691$

$-1.911943370889$

$-2.662772829873$

$-2.848354476903$

$-2.869357490622$

$-4.182621674211$

$-2.169517164078$

$-0.826247032602$

$-3.635301834708$

$-2.100473215277$

$-3.656497140730$

$-1.919161326972$

$-1.440923483892$

$-0.460307945588$

$-2.122309260996$

$-2.165500196904$

$-1.235710875826$

$-2.554890593351$

S-34 


$\begin{array}{lccr}\mathrm{H} & 0.798127659815 & 2.993526212161 & -2.910442609470 \\ \mathrm{C} & -1.108058599768 & 1.012403231359 & -3.221752821170 \\ \mathrm{H} & -0.395530580866 & 0.264052669318 & -3.589162432788 \\ \mathrm{H} & -2.074148201044 & 0.529583641555 & -3.064228211316 \\ \mathrm{H} & -1.219452277794 & 1.783363037787 & -3.992839427668 \\ \mathrm{C} & -3.016539797348 & 1.908088885017 & 1.028930108488 \\ \mathrm{H} & -3.482320425303 & 1.258577966113 & 1.775361195218 \\ \mathrm{H} & -1.932047993207 & 1.835145533931 & 1.158752356607 \\ \mathrm{H} & -3.315580339880 & 2.943615581581 & 1.232393101301 \\ \mathrm{C} & -4.979435647931 & 1.445767071507 & -0.432550095890 \\ \mathrm{H} & -5.436625362284 & 2.392771450158 & -0.122983163978 \\ \mathrm{H} & -5.318944720983 & 1.207166441556 & -1.446664115357 \\ \mathrm{H} & -5.323145060884 & 0.653875423596 & 0.242052493843 \\ * * * * * * * * * * * * * * * * * * * * * * * * * * * * * * * * * * * * * * * * * * * * * * * * * * * * * * * * * * * * * * * * * * * * * * * * * * * * * * * * * * * * * * * * * * *\end{array}$

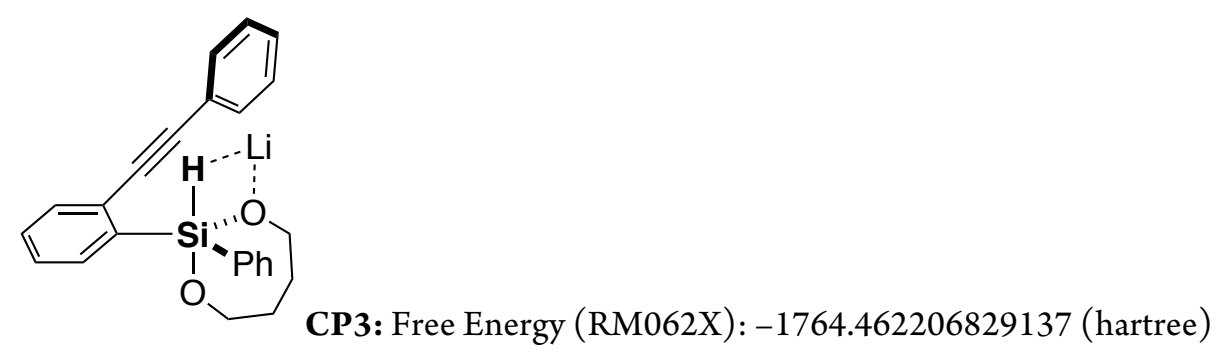

$\begin{array}{lccc}* * * * * * * * * * * * * * * * * * * * * * * * * * * * * * * * * * * * * * * * * * * * * * * * * * * * * * * * * * * * * * * * * * * * * * * * * * * * * * * * * * \\ \mathrm{C} & -2.541080099095 & 2.266200159660 & 1.689312439264 \\ \mathrm{C} & -1.388872893494 & 1.723294623117 & 1.105222459981 \\ \mathrm{C} & -0.235702518436 & 2.536210999381 & 1.091866465959 \\ \mathrm{C} & -0.243232634610 & 3.828255562253 & 1.644112251212 \\ \mathrm{C} & -1.400331593629 & 4.326341654151 & 2.234519134972 \\ \mathrm{C} & -2.554630051173 & 3.541183491382 & 2.257152956896 \\ \mathrm{H} & -3.452921721953 & 1.671064074834 & 1.695678225269 \\ \mathrm{H} & 0.657187334958 & 4.434953178115 & 1.599447445996 \\ \mathrm{H} & -1.402781418831 & 5.322279245525 & 2.667425007863 \\ \mathrm{H} & -3.463714111991 & 3.925270333752 & 2.712307983848 \\ \mathrm{C} & 0.961675229216 & 2.093226673795 & 0.424536608977 \\ \mathrm{C} & 1.959311201688 & 1.802757985658 & -0.207671036366 \\ \mathrm{C} & 3.067685452683 & 1.451032271769 & -1.050181723726 \\ \mathrm{C} & 2.812573076104 & 0.968281632949 & -2.344264221601 \\ \mathrm{C} & 4.394337497731 & 1.585465308667 & -0.613551677812 \\ \mathrm{C} & 3.866978134654 & 0.620159487910 & -3.183910017507 \\ \mathrm{H} & 1.782845766672 & 0.879733011765 & -2.680502851212 \\ \mathrm{C} & 5.444156809910 & 1.238815008130 & -1.460388029011 \\ \mathrm{H} & 4.593263636750 & 1.966663707043 & 0.383744857655 \\ \mathrm{C} & 5.185227645363 & 0.753170347380 & -2.743524405475 \\ \mathrm{H} & 3.659562379700 & 0.247708223349 & -4.182578584221 \\ \mathrm{H} & 6.468682442442 & 1.347372743178 & -1.116907717561 \\ \mathrm{H} & 6.007635389094 & 0.483422191090 & -3.398831635124 \\ \mathrm{Si} & -1.424538843594 & -0.109120801891 & 0.508205856729 \\ \mathrm{H} & -0.092711184682 & -0.293059012633 & 1.395386718808 \\ \mathrm{C} & -2.385490566867 & -1.252084400870 & 1.702898684582 \\ \mathrm{C} & -3.761782748710 & -1.507965956616 & 1.562554954036 \\ \mathrm{C} & -1.735951659137 & -1.858249780877 & 2.789398239688 \\ \mathrm{C} & -4.451548165217 & -2.325358356635 & 2.458777759011 \\ \mathrm{H} & -4.292046209622 & -1.054350750918 & 0.730365931085\end{array}$




\begin{tabular}{|c|c|c|c|}
\hline $\mathrm{C}$ & -2.413902552674 & -2.681080026114 & 3.692456272463 \\
\hline $\mathrm{H}$ & -0.671901799068 & -1.681715859908 & 2.933007640980 \\
\hline $\mathrm{C}$ & -3.778187607530 & -2.917298818003 & 3.529086036410 \\
\hline $\mathrm{H}$ & -5.515855978711 & -2.501253457325 & 2.323447719001 \\
\hline $\mathrm{H}$ & -1.877913482878 & -3.135732980280 & 4.521735648493 \\
\hline $\mathrm{H}$ & -4.312354184173 & -3.555182835934 & 4.228198942413 \\
\hline $\mathrm{C}$ & -0.714057439673 & -1.643128194471 & -1.847436025862 \\
\hline $\mathrm{C}$ & -2.651276154423 & -0.252186379803 & -2.928848229194 \\
\hline $\mathrm{C}$ & -2.873896785914 & 0.768643388346 & -1.800276581492 \\
\hline $\mathrm{H}$ & -2.895752001012 & 0.228832450404 & -3.885770246785 \\
\hline $\mathrm{H}$ & -3.379204530438 & -1.062972186451 & -2.792861602099 \\
\hline $\mathrm{O}$ & -0.390425154639 & -0.787983868187 & -0.740381805587 \\
\hline $\mathrm{O}$ & -2.783576248692 & 0.108205072613 & -0.552173126613 \\
\hline $\mathrm{Li}$ & 1.201435841407 & -0.609214065393 & 0.190919411076 \\
\hline $\mathrm{C}$ & 3.906117278321 & -1.876806258837 & -0.207541299045 \\
\hline $\mathrm{O}$ & 2.906434272744 & -1.269243924490 & 0.638546894635 \\
\hline $\mathrm{C}$ & 3.466445377583 & -0.950898248094 & 1.926005447004 \\
\hline $\mathrm{C}$ & 4.684061877274 & -1.854697529977 & 2.049143566624 \\
\hline $\mathrm{C}$ & 5.199885222557 & -1.864325616708 & 0.606693816002 \\
\hline $\mathrm{H}$ & 3.574876195961 & -2.895632775593 & -0.433790721631 \\
\hline $\mathrm{H}$ & 3.972456354649 & -1.301236008049 & -1.135519482533 \\
\hline $\mathrm{H}$ & 3.748085173521 & 0.110043651527 & 1.936939363869 \\
\hline $\mathrm{H}$ & 2.693393872609 & -1.126255290944 & 2.677503262313 \\
\hline $\mathrm{H}$ & 5.414759426860 & -1.473132075630 & 2.765648687944 \\
\hline $\mathrm{H}$ & 4.379025252374 & -2.859859468571 & 2.358549549069 \\
\hline $\mathrm{H}$ & 5.767052401314 & -0.950209100113 & 0.403241064328 \\
\hline $\mathrm{H}$ & 5.834417832909 & -2.723935628342 & 0.379792589948 \\
\hline $\mathrm{C}$ & -1.242513390650 & -0.836345037902 & -3.042666097978 \\
\hline $\mathrm{H}$ & -0.518156606990 & -0.037178162134 & -3.251465535244 \\
\hline $\mathrm{H}$ & -1.224137832789 & -1.500844759939 & -3.916983901900 \\
\hline $\mathrm{C}$ & 0.615013622627 & -2.294830792847 & -2.240853548783 \\
\hline $\mathrm{H}$ & 1.348794931087 & -1.530110588014 & -2.524807587692 \\
\hline $\mathrm{H}$ & 1.018297696003 & -2.865715268140 & -1.395708934281 \\
\hline $\mathrm{H}$ & 0.486998238232 & -2.980271838500 & -3.084858642511 \\
\hline $\mathrm{C}$ & -1.694225446002 & -2.732645147067 & -1.409018465658 \\
\hline $\mathrm{H}$ & -1.255323768251 & -3.321660169057 & -0.595602371235 \\
\hline $\mathrm{H}$ & -2.633041085661 & -2.303199453535 & -1.053403856304 \\
\hline $\mathrm{H}$ & -1.909026236920 & -3.405296453356 & -2.247559201498 \\
\hline $\mathrm{C}$ & -1.898426704676 & 1.947396452878 & -1.948155671503 \\
\hline $\mathrm{H}$ & -2.152444780347 & 2.752434847563 & -1.251752419346 \\
\hline $\mathrm{H}$ & -0.861687224969 & 1.649644968473 & -1.756688356550 \\
\hline $\mathrm{H}$ & -1.954244968712 & 2.343242161979 & -2.969468052580 \\
\hline $\mathrm{C}$ & -4.305557386911 & 1.304650067422 & -1.896957561149 \\
\hline $\mathrm{H}$ & -4.473976897506 & 1.833359810815 & -2.842119100574 \\
\hline $\mathrm{H}$ & -5.021978392172 & 0.478395019418 & -1.825440640447 \\
\hline $\mathrm{H}$ & -4.493496151679 & 1.997177157544 & -1.069111355283 \\
\hline
\end{tabular}




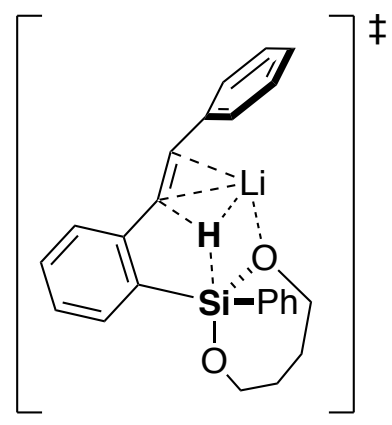

TS2: Free Energy (RM062X): -1764.438279508539 (hartree)

\begin{tabular}{|c|c|c|c|}
\hline $\mathrm{C}$ & -2.684437617696 & 2.676374210423 & 0.836099177923 \\
\hline $\mathrm{C}$ & -1.517101992321 & 1.963335848702 & 0.534048428408 \\
\hline $\mathrm{C}$ & -0.287683723428 & 2.618752867889 & 0.671428626628 \\
\hline $\mathrm{C}$ & -0.222709665572 & 3.964778309617 & 1.058784579049 \\
\hline $\mathrm{C}$ & -1.395922038472 & 4.654212940101 & 1.348397549667 \\
\hline $\mathrm{C}$ & -2.632183444121 & 4.007598280104 & 1.247214043750 \\
\hline $\mathrm{H}$ & -3.646144540901 & 2.174724688040 & 0.753250665742 \\
\hline $\mathrm{H}$ & 0.745291886046 & 4.451950307432 & 1.140902383551 \\
\hline $\mathrm{H}$ & -1.348266504248 & 5.693206902852 & 1.662251376665 \\
\hline $\mathrm{H}$ & -3.546813661897 & 4.542097366247 & 1.489165732721 \\
\hline $\mathrm{C}$ & 0.947223535816 & 1.867912546646 & 0.399214567594 \\
\hline $\mathrm{C}$ & 2.174461030921 & 1.961789806949 & 0.167741061286 \\
\hline $\mathrm{C}$ & 3.532245284791 & 1.639429187174 & -0.101602249729 \\
\hline $\mathrm{C}$ & 4.095791363316 & 1.869070657390 & -1.377346811336 \\
\hline $\mathrm{C}$ & 4.365244303119 & 1.095401543087 & 0.901353516068 \\
\hline $\mathrm{C}$ & 5.424025924474 & 1.547920134879 & -1.638292738211 \\
\hline $\mathrm{H}$ & 3.478250926211 & 2.308602630632 & -2.156016948890 \\
\hline $\mathrm{C}$ & 5.695026371518 & 0.789093991025 & 0.631520498171 \\
\hline $\mathrm{H}$ & 3.952807017396 & 0.918074380658 & 1.890783862869 \\
\hline $\mathrm{C}$ & 6.235703112489 & 1.004809179464 & -0.638848145065 \\
\hline $\mathrm{H}$ & 5.830724271196 & 1.729596817473 & -2.629409389417 \\
\hline $\mathrm{H}$ & 6.314572152297 & 0.370492130550 & 1.420375108163 \\
\hline $\mathrm{H}$ & 7.273310478183 & 0.760983857862 & -0.844199888616 \\
\hline $\mathrm{Si}$ & -1.491965647689 & 0.097223565568 & 0.118750354649 \\
\hline $\mathrm{H}$ & 0.214523562740 & 0.477608386344 & 0.457800689226 \\
\hline $\mathrm{C}$ & -1.575614321242 & -0.940850541752 & 1.710654803921 \\
\hline $\mathrm{C}$ & -2.616748797348 & -1.850675356090 & 1.956817410750 \\
\hline $\mathrm{C}$ & -0.631814027240 & -0.753772645318 & 2.733127733203 \\
\hline $\mathrm{C}$ & -2.693414092412 & -2.567567960260 & 3.152224138306 \\
\hline $\mathrm{H}$ & -3.388337294033 & -1.986804780663 & 1.203585566456 \\
\hline $\mathrm{C}$ & -0.706025319239 & -1.453116985788 & 3.938179980725 \\
\hline $\mathrm{H}$ & 0.181158359596 & -0.045552665514 & 2.580591327805 \\
\hline $\mathrm{C}$ & -1.734971865412 & -2.372455174538 & 4.147042010162 \\
\hline $\mathrm{H}$ & -3.506668471533 & -3.270874560425 & 3.311145339547 \\
\hline $\mathrm{H}$ & 0.037886844693 & -1.282452555757 & 4.711951027029 \\
\hline $\mathrm{H}$ & -1.794025699294 & -2.925470188442 & 5.080458095965 \\
\hline $\mathrm{C}$ & -1.083208781285 & -1.639814719621 & -2.142662111421 \\
\hline $\mathrm{C}$ & -3.596328664518 & -1.017287796795 & -2.561961136451 \\
\hline $\mathrm{C}$ & -3.761651333147 & 0.163479175713 & -1.594045332459 \\
\hline $\mathrm{H}$ & -4.274228274421 & -0.856764678254 & -3.410737933654 \\
\hline $\mathrm{H}$ & -3.949074694875 & -1.922405159728 & -2.050617021011 \\
\hline $\mathrm{O}$ & -0.701336983398 & -0.530861136352 & -1.297087734489 \\
\hline $\mathrm{O}$ & -3.108310959580 & -0.136713308224 & -0.365701114038 \\
\hline $\mathrm{Li}$ & 1.119315859164 & 0.068993900948 & -1.111482789212 \\
\hline
\end{tabular}




\begin{tabular}{|c|c|c|c|}
\hline $\mathrm{C}$ & 3.569782921287 & -1.747087655651 & -0.944817099521 \\
\hline $\mathrm{O}$ & 2.299209867067 & -1.263149317491 & -0.464758850908 \\
\hline $\mathrm{C}$ & 2.001014822264 & -1.828324766254 & 0.823117310932 \\
\hline $\mathrm{C}$ & 2.868304425609 & -3.075429281353 & 0.906146871558 \\
\hline $\mathrm{C}$ & 4.135335820126 & -2.609832738406 & 0.182610551284 \\
\hline $\mathrm{H}$ & 3.382254752180 & -2.334615442512 & -1.851788435029 \\
\hline $\mathrm{H}$ & 4.200946444191 & -0.889123237705 & -1.190284158578 \\
\hline $\mathrm{H}$ & 2.263560128925 & -1.096299042843 & 1.598863285423 \\
\hline $\mathrm{H}$ & 0.925283721065 & -2.015752417459 & 0.864829516618 \\
\hline $\mathrm{H}$ & 3.047938751269 & -3.390655924729 & 1.936479830756 \\
\hline $\mathrm{H}$ & 2.394308670453 & -3.901481537409 & 0.363652422852 \\
\hline $\mathrm{H}$ & 4.748438021961 & -1.997186287803 & 0.852160180166 \\
\hline $\mathrm{H}$ & 4.747395669188 & -3.431574820597 & -0.196217497603 \\
\hline $\mathrm{C}$ & -2.193595749505 & -1.243629904563 & -3.125290307321 \\
\hline $\mathrm{H}$ & -1.856602031946 & -0.353073490325 & -3.671701879063 \\
\hline $\mathrm{H}$ & -2.260521473994 & -2.048872321817 & -3.868627089740 \\
\hline $\mathrm{C}$ & 0.174890864686 & -1.983469295681 & -2.942714624656 \\
\hline $\mathrm{H}$ & 0.502532571609 & -1.119058784785 & -3.534683378842 \\
\hline $\mathrm{H}$ & 0.983397847738 & -2.276052962974 & -2.264872960149 \\
\hline $\mathrm{H}$ & -0.016435258361 & -2.812681484871 & -3.630860576506 \\
\hline $\mathrm{C}$ & -1.475261588760 & -2.841006747833 & -1.285120607665 \\
\hline $\mathrm{H}$ & -0.642809466580 & -3.120342143831 & -0.629040846118 \\
\hline $\mathrm{H}$ & -2.344493192867 & -2.622502125524 & -0.662309563489 \\
\hline $\mathrm{H}$ & -1.715180957951 & -3.697809410251 & -1.924837351037 \\
\hline $\mathrm{C}$ & -3.256319614572 & 1.461494326736 & -2.238462759426 \\
\hline $\mathrm{H}$ & -3.532094464438 & 2.331339088205 & -1.636977095757 \\
\hline $\mathrm{H}$ & -2.167758765718 & 1.462533021096 & -2.353498570637 \\
\hline $\mathrm{H}$ & -3.702363035567 & 1.576007788737 & -3.233350666979 \\
\hline $\mathrm{C}$ & -5.245888090547 & 0.314009342571 & -1.254189087966 \\
\hline $\mathrm{H}$ & -5.833270412849 & 0.553209935060 & -2.147278786947 \\
\hline $\mathrm{H}$ & -5.628931356388 & -0.616381210671 & -0.820908114528 \\
\hline $\mathrm{H}$ & -5.384417708236 & 1.116408450692 & -0.522177973127 \\
\hline
\end{tabular}

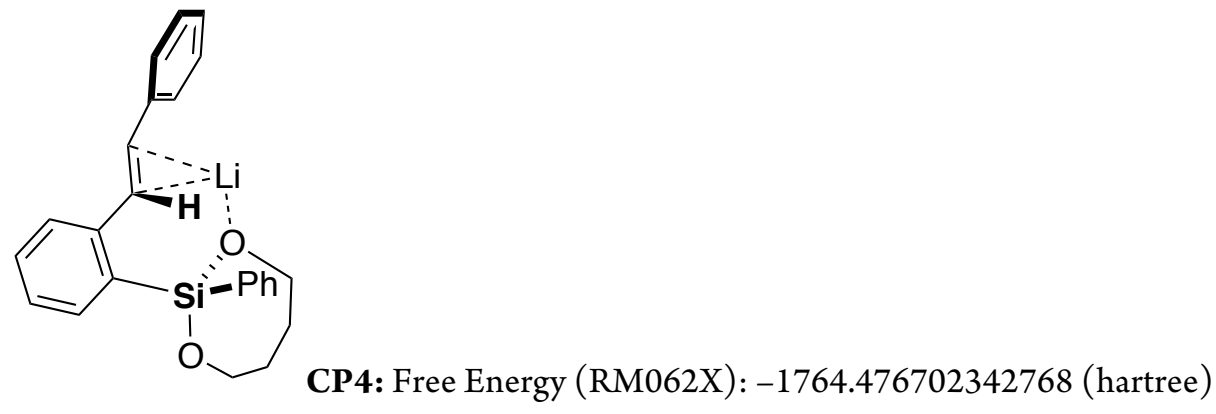

$\begin{array}{lccc}* * * * * * * * * * * * * * * * * * * * * * * * * * * * * * * * * * * * * * * * * * * * * * * * * * * * * * * * * * * * * * * * * * * * * * * * * * * * * * * \\ \mathrm{C} & -2.574210378488 & 2.558429039319 & 0.941546812932 \\ \mathrm{C} & -1.410795115747 & 1.852979335484 & 0.577498172252 \\ \mathrm{C} & -0.174776244027 & 2.534066969305 & 0.572761841115 \\ \mathrm{C} & -0.161500212084 & 3.907748546313 & 0.872009906644 \\ \mathrm{C} & -1.324351122727 & 4.593551885411 & 1.202241911345 \\ \mathrm{C} & -2.542842750139 & 3.913201213040 & 1.255955132386 \\ \mathrm{H} & -3.529521606738 & 2.036936170547 & 0.964880886299 \\ \mathrm{H} & 0.796704137800 & 4.418274084644 & 0.855987567475 \\ \mathrm{H} & -1.279239264605 & 5.653301670522 & 1.439043579123 \\ \mathrm{H} & -3.455352354016 & 4.432013434076 & 1.535236555192 \\ & & \mathrm{~S}-38 & \end{array}$




\begin{tabular}{|c|c|}
\hline $\mathrm{C}$ & 1.124359811040 \\
\hline $\mathrm{C}$ & 2.171820404232 \\
\hline $\mathrm{C}$ & 3.480014561400 \\
\hline $\mathrm{C}$ & 4.273361163613 \\
\hline $\mathrm{C}$ & 4.067763873527 \\
\hline $\mathrm{C}$ & 5.564216836199 \\
\hline $\mathrm{H}$ & 3.864354945006 \\
\hline $\mathrm{C}$ & 5.367178077890 \\
\hline $\mathrm{H}$ & 3.494096885423 \\
\hline $\mathrm{C}$ & 6.126485291258 \\
\hline $\mathrm{H}$ & 6.143674327799 \\
\hline $\mathrm{H}$ & 5.792933149354 \\
\hline $\mathrm{H}$ & 7.141774668843 \\
\hline Si & -1.720850924572 \\
\hline $\mathrm{H}$ & 1.185994055357 \\
\hline $\mathrm{C}$ & -1.599701834582 \\
\hline $\mathrm{C}$ & -2.423337920026 \\
\hline $\mathrm{C}$ & -0.686783666606 \\
\hline $\mathrm{C}$ & -2.311606794832 \\
\hline $\mathrm{H}$ & -3.175875107825 \\
\hline $\mathrm{C}$ & -0.562429804453 \\
\hline $\mathrm{H}$ & -0.061700325771 \\
\hline $\mathrm{C}$ & -1.371182621763 \\
\hline $\mathrm{H}$ & -2.961017657052 \\
\hline $\mathrm{H}$ & 0.156934924074 \\
\hline $\mathrm{H}$ & -1.280478295688 \\
\hline $\mathrm{C}$ & -1.017779350533 \\
\hline $\mathrm{C}$ & -3.517028846133 \\
\hline $\mathrm{C}$ & -3.868922741886 \\
\hline $\mathrm{H}$ & -4.101404548362 \\
\hline $\mathrm{H}$ & -3.873309689206 \\
\hline $\mathrm{O}$ & -0.682784392702 \\
\hline $\mathrm{O}$ & -3.272576748718 \\
\hline $\mathrm{Li}$ & 1.213283647919 \\
\hline $\mathrm{C}$ & 3.495951189282 \\
\hline $\mathrm{O}$ & 2.244639697702 \\
\hline $\mathrm{C}$ & 1.867828684449 \\
\hline $\mathrm{C}$ & 2.679329634400 \\
\hline $\mathrm{C}$ & 3.989841888907 \\
\hline $\mathrm{H}$ & 3.294379771222 \\
\hline $\mathrm{H}$ & 4.172119638932 \\
\hline $\mathrm{H}$ & 2.125819452113 \\
\hline $\mathrm{H}$ & 0.785272878095 \\
\hline $\mathrm{H}$ & 2.803908640491 \\
\hline $\mathrm{H}$ & 2.194847133300 \\
\hline $\mathrm{H}$ & 4.596412865257 \\
\hline $\mathrm{H}$ & 4.588029391616 \\
\hline $\mathrm{C}$ & -2.048257650709 \\
\hline $\mathrm{H}$ & -1.686730931993 \\
\hline $\mathrm{H}$ & -2.009972318131 \\
\hline $\mathrm{C}$ & 0.272501559630 \\
\hline $\mathrm{H}$ & 0.649994598141 \\
\hline $\mathrm{H}$ & 1.037045229634 \\
\hline $\mathrm{H}$ & 0.084635100762 \\
\hline $\mathrm{C}$ & -1.491234577375 \\
\hline $\mathrm{H}$ & -0.727469233654 \\
\hline
\end{tabular}

1.851363803265

2.440019506027

1.780900127271

1.771869477808

1.245188241573

1.248970208002

2.218038568201

0.742234377038

1.257186208102

0.735443045148

1.259283161627

0.351723660268

0.349783522153

0.059039535423

0.845300950789

$-0.970487112478$

$-2.080146500472$

$-0.599744085014$

$-2.816687966933$

$-2.366430243138$

$-1.334925765541$

0.281382856435

$-2.451752392603$

$-3.670977674258$

$-1.031203088806$

$-3.026011421308$

$-1.516223139966$

$-0.908487914688$

0.181825432460

$-0.711539747844$

$-1.869516516883$

$-0.446059994372$

$-0.156597805249$

0.235657224762

$-1.718390723827$

$-1.183970280986$

$-1.828780883565$

$-3.115629598952$

$-2.658917822036$

$-2.258478323357$

$-0.885783967514$

$-1.169070425649$

$-1.977108711781$

$-3.501218782238$

$-3.885887717466$

$-2.103890857112$

$-3.480520325736$

$-1.021982421330$

$-0.067562674367$

$-1.733373069472$

$-1.851867066756$

$-0.982261824896$

$-2.201890650398$

$-2.642840161164$

$-2.759339578940$

$-3.091690822691$
0.323203304298

$-0.299589145032$

$-0.311683358766$

$-1.481708801552$

0.858525456985

$-1.489132472132$

$-2.385938845356$

0.856949789826

1.783137786789

$-0.316407687295$

$-2.409006364319$

1.778621544604

$-0.313914884434$

0.153040556358

0.795079774616

1.715819212219

1.961735593941

2.716464096957

3.140718759641

1.231614201694

3.894960061825

2.580441982889

4.105168969858

3.309304276507

4.650118029749

5.022734036269

$-2.036867333903$

$-2.650177117134$

$-1.632874095659$

$-3.557700972097$

$-2.258789399871$

$-1.090478574430$

$-0.367161636005$

$-1.230974405112$

$-0.899931607078$

$-0.418776094691$

0.808934167280

0.842436031370

0.195792266621

$-1.834209333777$

$-1.097438115759$

1.648867017571

0.794882521748

1.856856883968

0.230988378109

0.919545234056

$-0.204800118116$

$-3.067156756589$

$-3.469918826359$

$-3.901198853666$

$-2.781139796007$

$-3.333136028517$

$-2.084388891388$

$-3.512323373946$

$-1.289728795071$

$-0.578620650452$ 


$\begin{array}{lccc}\mathrm{H} & -2.420356979422 & -2.582439802720 & -0.746048651031 \\ \mathrm{H} & -1.671716112817 & -3.568406525897 & -2.005575191595 \\ \mathrm{C} & -3.422470615165 & 1.560993029202 & -2.123601476838 \\ \mathrm{H} & -3.788551096485 & 2.348511759216 & -1.459842712925 \\ \mathrm{H} & -2.332568823293 & 1.649832927165 & -2.178376926078 \\ \mathrm{H} & -3.826861303978 & 1.737725577664 & -3.126202345808 \\ \mathrm{C} & -5.374713123495 & 0.183394236541 & -1.385146198361 \\ \mathrm{H} & -5.916965106788 & 0.441762796683 & -2.300239313368 \\ \mathrm{H} & -5.704855020535 & -0.804467144498 & -1.047969754830 \\ \mathrm{H} & -5.625674195854 & 0.916221502496 & -0.611771716124 \\ * * * * * * * * * * * * * * * * * * * * * * * * * * * * * * * * * * * * * * * * * * * * * * * * * * * * * * * * * * * * * * * * * * * * * * * * * * * * * * * * * * * * * * * * * * * * *\end{array}$

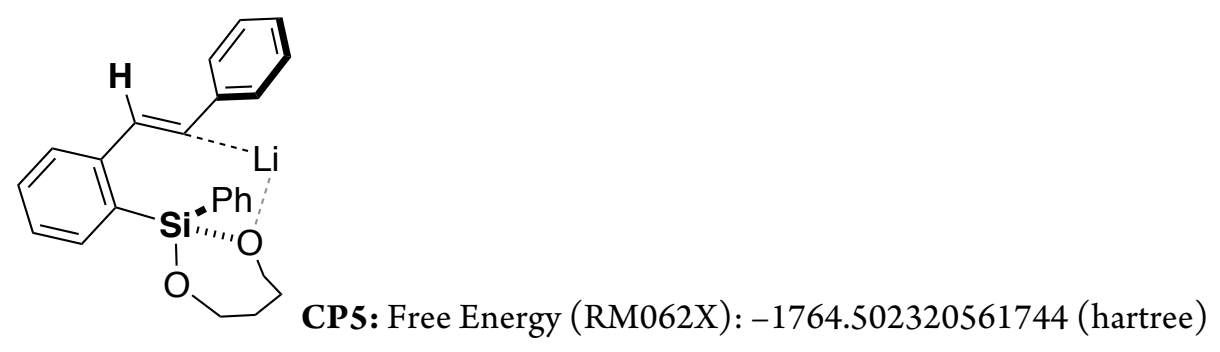

\begin{tabular}{|c|c|c|c|}
\hline $\mathrm{C}$ & -3.509174123433 & 1.902569307865 & -0.139039405083 \\
\hline $\mathrm{C}$ & -2.109716846697 & 1.764604732467 & -0.106538884794 \\
\hline $\mathrm{C}$ & -1.314697251315 & 2.932208184193 & -0.208350455367 \\
\hline $\mathrm{C}$ & -1.953926592197 & 4.181742615361 & -0.299005348790 \\
\hline $\mathrm{C}$ & -3.339001312166 & 4.293915945615 & -0.338275148580 \\
\hline $\mathrm{C}$ & -4.127573942242 & 3.144759252235 & -0.265068072448 \\
\hline $\mathrm{H}$ & -4.127224969050 & 1.014241261435 & -0.041609418322 \\
\hline $\mathrm{H}$ & -1.338478622182 & 5.077491428065 & -0.352101252908 \\
\hline $\mathrm{H}$ & -3.802652258227 & 5.273268681284 & -0.422130938848 \\
\hline $\mathrm{H}$ & -5.211250442751 & 3.216023781051 & -0.286378202041 \\
\hline $\mathrm{C}$ & 0.160688353790 & 2.907119433089 & -0.193481904214 \\
\hline $\mathrm{C}$ & 0.927526745634 & 1.863604467259 & -0.562104106441 \\
\hline $\mathrm{C}$ & 2.381785368814 & 2.022096086461 & -0.409600864147 \\
\hline $\mathrm{C}$ & 3.193963487997 & 2.499764744482 & -1.459580862123 \\
\hline $\mathrm{C}$ & 3.030751065790 & 1.623740207825 & 0.778523632686 \\
\hline $\mathrm{C}$ & 4.580430656147 & 2.573280101593 & -1.330394261099 \\
\hline $\mathrm{H}$ & 2.720844537214 & 2.813334750112 & -2.388104151853 \\
\hline $\mathrm{C}$ & 4.417265106880 & 1.689514395071 & 0.902284373835 \\
\hline $\mathrm{H}$ & 2.426975421824 & 1.251409019484 & 1.603768724553 \\
\hline $\mathrm{C}$ & 5.205774084699 & 2.158385030428 & -0.151945975641 \\
\hline $\mathrm{H}$ & 5.176823154854 & 2.953000892389 & -2.156459119199 \\
\hline $\mathrm{H}$ & 4.886725219094 & 1.371845658428 & 1.830715164514 \\
\hline $\mathrm{H}$ & 6.286303755438 & 2.206370695998 & -0.054271105871 \\
\hline $\mathrm{Si}$ & -1.417555852343 & 0.050913546558 & 0.185596133681 \\
\hline $\mathrm{H}$ & 0.606898012606 & 3.851290748508 & 0.155415871125 \\
\hline $\mathrm{C}$ & -0.213493124763 & -0.086113622159 & 1.611961184669 \\
\hline $\mathrm{C}$ & -0.399942824393 & 0.736356410024 & 2.733545125600 \\
\hline $\mathrm{C}$ & 0.814926375067 & -1.035277214871 & 1.658995217328 \\
\hline $\mathrm{C}$ & 0.412322761981 & 0.615413030784 & 3.860219063474 \\
\hline $\mathrm{H}$ & -1.184115604281 & 1.492004641749 & 2.724199819572 \\
\hline $\mathrm{C}$ & 1.622853351139 & -1.174928146182 & 2.788172656248 \\
\hline $\mathrm{H}$ & 0.998973503544 & -1.667626039802 & 0.794257878429 \\
\hline $\mathrm{C}$ & 1.423406081920 & -0.347613318789 & 3.893047405313 \\
\hline \multirow[t]{2}{*}{$\mathrm{H}$} & 0.256125329069 & 1.268941197703 & 4.713865424770 \\
\hline & & S-40 & \\
\hline
\end{tabular}




\begin{tabular}{|c|c|c|c|}
\hline $\mathrm{H}$ & 2.413847605878 & -1.921015449455 & 2.804134295803 \\
\hline $\mathrm{H}$ & 2.053444916158 & -0.448347698557 & 4.772067007435 \\
\hline $\mathrm{C}$ & -1.937231152628 & -3.031757240625 & 0.028889083494 \\
\hline $\mathrm{C}$ & -2.960767272428 & -2.200497119926 & 0.817858553695 \\
\hline $\mathrm{H}$ & -2.118286592359 & -4.086372577623 & 0.271776811963 \\
\hline $\mathrm{H}$ & -0.936798806409 & -2.804193690732 & 0.419531703179 \\
\hline $\mathrm{O}$ & -0.769768161086 & -0.761121739941 & -1.155968840738 \\
\hline $\mathrm{O}$ & -2.798166903471 & -0.799878221456 & 0.575039556274 \\
\hline $\mathrm{Li}$ & 0.907180222226 & 0.026540041725 & -1.603977272751 \\
\hline $\mathrm{C}$ & 2.051704011201 & -2.746777962995 & -1.278162949788 \\
\hline $\mathrm{O}$ & 2.271850242915 & -1.354028450225 & -1.534605780937 \\
\hline $\mathrm{C}$ & 3.676986923551 & -1.132226984957 & -1.343861292050 \\
\hline $\mathrm{C}$ & 3.992547135404 & -1.913074527356 & -0.074677111524 \\
\hline $\mathrm{C}$ & 3.075082668686 & -3.149165615016 & -0.194566181223 \\
\hline $\mathrm{H}$ & 1.007817628332 & -2.865024060786 & -0.975339696263 \\
\hline $\mathrm{H}$ & 2.215492104115 & -3.305970088319 & -2.208058944116 \\
\hline $\mathrm{H}$ & 4.217917161837 & -1.533429695578 & -2.212444072310 \\
\hline $\mathrm{H}$ & 3.844614922147 & -0.057797586151 & -1.266329246126 \\
\hline $\mathrm{H}$ & 5.050616901003 & -2.174214099521 & 0.002497670732 \\
\hline $\mathrm{H}$ & 3.719703391911 & -1.303514691152 & 0.792486315747 \\
\hline $\mathrm{H}$ & 3.632735731666 & -4.034613600457 & -0.509119128952 \\
\hline $\mathrm{H}$ & 2.586728819266 & -3.386631941104 & 0.754779021693 \\
\hline $\mathrm{C}$ & -2.771746705421 & -2.454165541747 & 2.315268271948 \\
\hline $\mathrm{H}$ & -2.972876605383 & -3.502803808086 & 2.559694732618 \\
\hline $\mathrm{H}$ & -1.748925864044 & -2.213515877204 & 2.624251468695 \\
\hline $\mathrm{H}$ & -3.461620021654 & -1.823823321418 & 2.885558946227 \\
\hline $\mathrm{C}$ & -4.393354907831 & -2.543957770736 & 0.412360826136 \\
\hline $\mathrm{H}$ & -5.094821647643 & -1.944059119915 & 1.000914792070 \\
\hline $\mathrm{H}$ & -4.570323090354 & -2.332748452550 & -0.646174668271 \\
\hline $\mathrm{H}$ & -4.601861858338 & -3.603640414617 & 0.594586806077 \\
\hline $\mathrm{C}$ & -0.708512342698 & -1.701160330792 & -3.343063288876 \\
\hline $\mathrm{H}$ & -0.502510299008 & -0.735522396212 & -3.818524691497 \\
\hline $\mathrm{H}$ & 0.241194898459 & -2.184501116614 & -3.095425963963 \\
\hline $\mathrm{H}$ & -1.229377947077 & -2.332809031515 & -4.069674549580 \\
\hline $\mathrm{C}$ & -2.820406804344 & -0.723430845471 & -2.501115962263 \\
\hline $\mathrm{H}$ & -2.530252659538 & 0.273276005043 & -2.851478650418 \\
\hline $\mathrm{H}$ & -3.328693882250 & -1.242653262665 & -3.320437810089 \\
\hline $\mathrm{H}$ & -3.525583233282 & -0.610216558251 & -1.676508814037 \\
\hline $\mathrm{C}$ & -1.574746232587 & -1.517942556513 & -2.097549727997 \\
\hline $\mathrm{C}$ & -1.922426598914 & -2.905623667098 & -1.499414872012 \\
\hline $\mathrm{H}$ & -2.882695312339 & -3.230928366596 & -1.918081487111 \\
\hline $\mathrm{H}$ & -1.181197710428 & -3.630258136092 & -1.861083274591 \\
\hline
\end{tabular}

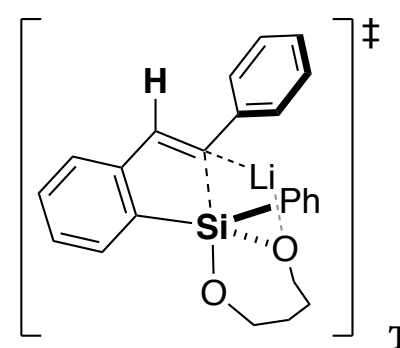

TS3: Free Energy (RM062X): -1764.498470560655 (hartree)

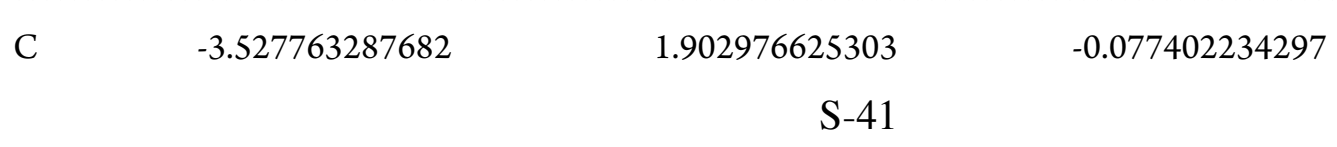




\begin{tabular}{|c|c|}
\hline C & -2.127286169580 \\
\hline $\mathrm{C}$ & -1.402128756689 \\
\hline $\mathrm{C}$ & -2.079519444325 \\
\hline $\mathrm{C}$ & -3.470739664685 \\
\hline $\mathrm{C}$ & -4.200787573848 \\
\hline $\mathrm{H}$ & -4.098234361341 \\
\hline $\mathrm{H}$ & -1.503618416878 \\
\hline $\mathrm{H}$ & -3.984169475273 \\
\hline $\mathrm{H}$ & -5.286525993536 \\
\hline $\mathrm{C}$ & 0.065679824741 \\
\hline $\mathrm{C}$ & 0.729687449789 \\
\hline $\mathrm{C}$ & 2.205418669757 \\
\hline C & 2.955497766037 \\
\hline $\mathrm{C}$ & 2.919981971792 \\
\hline C & 4.346350651864 \\
\hline $\mathrm{H}$ & 2.429207647617 \\
\hline $\mathrm{C}$ & 4.308347258153 \\
\hline $\mathrm{H}$ & 2.369184003877 \\
\hline $\mathrm{C}$ & 5.033765555302 \\
\hline $\mathrm{H}$ & 4.892716112453 \\
\hline $\mathrm{H}$ & 4.828577814673 \\
\hline $\mathrm{H}$ & 6.115986537896 \\
\hline $\mathrm{Si}$ & -1.236484275427 \\
\hline $\mathrm{H}$ & 0.578979693440 \\
\hline $\mathrm{C}$ & -0.147198061983 \\
\hline C & -0.387210138565 \\
\hline $\mathrm{C}$ & 0.873348277886 \\
\hline $\mathrm{C}$ & 0.368131920348 \\
\hline $\mathrm{H}$ & -1.173394586899 \\
\hline $\mathrm{C}$ & 1.619363555798 \\
\hline $\mathrm{H}$ & 1.102721368085 \\
\hline $\mathrm{C}$ & 1.370454081476 \\
\hline $\mathrm{H}$ & 0.170506282017 \\
\hline $\mathrm{H}$ & 2.402799280418 \\
\hline $\mathrm{H}$ & 1.953217920891 \\
\hline $\mathrm{C}$ & -1.834810024049 \\
\hline $\mathrm{C}$ & -2.821981942603 \\
\hline $\mathrm{H}$ & -2.034231117900 \\
\hline $\mathrm{H}$ & -0.823296817681 \\
\hline $\mathrm{O}$ & -0.670609810547 \\
\hline $\mathrm{O}$ & -2.629465079591 \\
\hline $\mathrm{Li}$ & 1.014844804564 \\
\hline $\mathrm{C}$ & 2.055902672659 \\
\hline $\mathrm{O}$ & 2.327898995521 \\
\hline $\mathrm{C}$ & 3.734535710109 \\
\hline $\mathrm{C}$ & 4.003497605699 \\
\hline $\mathrm{C}$ & 3.058216676968 \\
\hline $\mathrm{H}$ & 1.007627076297 \\
\hline $\mathrm{H}$ & 2.202841793288 \\
\hline $\mathrm{H}$ & 4.280980631927 \\
\hline $\mathrm{H}$ & 3.929343971627 \\
\hline $\mathrm{H}$ & 5.052536400889 \\
\hline $\mathrm{H}$ & 3.727380229392 \\
\hline $\mathrm{H}$ & 3.599486777035 \\
\hline $\mathrm{H}$ & 2.554030210817 \\
\hline $\mathrm{C}$ & -2.629674194141 \\
\hline
\end{tabular}

1.830774278126

3.023198728731

4.243053259528

4.291558966727

3.115755519219

0.993864512126

5.156406270695

5.242314620415

3.143674011673

2.969211007262

1.801483411926

1.899579916855

2.380605405563

1.512954874695

2.480573626857

2.691721437273

1.615801346235

1.153295952138

2.095230142633

2.864495481659

1.321810978099

2.171611772488

0.187375727063

3.935643656213

$-0.074846252823$

0.661339205362

$-1.033793350440$

0.451947430360

1.415101160912

$-1.267728556299$

$-1.599298709129$

$-0.521045638361$

1.041114028585

$-2.022406978418$

$-0.692672419487$

$-2.954397969018$

$-2.082869830996$

$-4.002814816912$

$-2.741574601704$

$-0.694753423869$

$-0.701053990694$

0.010191344187

$-2.780351553844$

$-1.395460542620$

$-1.214470816278$

$-2.005910544388$

$-3.217739158394$

$-2.857305759675$

$-3.345238028722$

$-1.628351188926$

$-0.145164107093$

$-2.294525235349$

$-1.393268175470$

$-4.111577877972$

$-3.454883714436$

$-2.337360245695$
$-0.103071414242$

$-0.303141246463$

$-0.443486618421$

$-0.418635340900$

$-0.238854383000$

0.088032818336

$-0.579049785153$

$-0.535472797136$

$-0.209874741112$

$-0.333026268851$

$-0.412259360874$

$-0.348354877489$

$-1.442030638239$

0.804863523607

$-1.384715035367$

$-2.342279159363$

0.861087658916

1.669712635644

$-0.233076942344$

$-2.242357354034$

1.769592129540

$-0.185724877858$

0.150843603644

$-0.236635395494$

1.659210102694

2.828989379748

1.690173192472

3.982788246473

2.840285417028

2.845728442804

0.789699277675

3.997534650633

4.874174757667

2.846204189053

4.897924512153

0.025301939891

0.815940776289

0.282420376482

0.393867143230

$-1.203434640307$

0.547037901929

$-1.568681146329$

$-1.283539961354$

$-1.541496803936$

$-1.322668263219$

$-0.049451366996$

$-0.193928297437$

$-0.984538724129$

$-2.212381335741$

$-2.181540808534$

$-1.240855102352$

0.049193071171

0.814745891247

$-0.513265548441$

0.747099875710

2.315376837644 


$\begin{array}{lccc}\mathrm{H} & -2.886450122879 & -3.372263502755 & 2.568527116292 \\ \mathrm{H} & -1.594002347892 & -2.151269310041 & 2.616760661224 \\ \mathrm{H} & -3.279922906997 & -1.666857297102 & 2.887247977055 \\ \mathrm{C} & -4.270060402818 & -2.402640882105 & 0.436291556367 \\ \mathrm{H} & -4.948735263608 & -1.783326528250 & 1.032293456677 \\ \mathrm{H} & -4.461408287133 & -2.193889003442 & -0.620276292280 \\ \mathrm{H} & -4.500276417675 & -3.456396148568 & 0.629051936003 \\ \mathrm{C} & -0.663752671829 & -1.658658132339 & -3.374567071027 \\ \mathrm{H} & -0.456225542229 & -0.698048352165 & -3.860764238355 \\ \mathrm{H} & 0.286345470899 & -2.149157981089 & -3.141364693507 \\ \mathrm{H} & -1.204633664934 & -2.290944060792 & -4.086158451687 \\ \mathrm{C} & -2.747550799654 & -0.652217685426 & -2.498600015627 \\ \mathrm{H} & -2.451451038303 & 0.335349065349 & -2.870384915228 \\ \mathrm{H} & -3.282161898595 & -1.175678635035 & -3.298642466556 \\ \mathrm{H} & -3.426422002795 & -0.515239315361 & -1.656540362037 \\ \mathrm{C} & -1.502529887700 & -1.455788470860 & -2.111872921582 \\ \mathrm{C} & -1.851643081981 & -2.837740916113 & -1.502112525357 \\ \mathrm{H} & -2.826127868701 & -3.151926280420 & -1.896212905677 \\ \mathrm{H} & -1.129905273061 & -3.573623775381 & -1.881072726967 \\ * * * * * * * * * * * * * * * * * * * * * * * * * * * * * * * * * * * * * * * * * * * * * * * * * * * * * * * * * * * * * * * * * * * * * * * * * * * * * * * * * * * * *\end{array}$<smiles>[PH3+][C@]1(c2ccccc2)C(c2ccccc2)=Cc2ccccc21</smiles>

CP6: Free Energy (RM062X): -1764.502127124008 (hartree)

$\begin{array}{lccc}* * * * * * * * * * * * * * * * * * * * * * * * * * * * * * * * * * * * * * * * * * * * * * * * * * * * * * * * * * * * * * * * * * * * * * * * * * * * * * * \\ \mathrm{C} & -3.569394938053 & 1.921203237128 & 0.095970659105 \\ \mathrm{C} & -2.177997652918 & 1.891702741240 & -0.031278107770 \\ \mathrm{C} & -1.516976879192 & 3.089761853573 & -0.355268165333 \\ \mathrm{C} & -2.224681301861 & 4.282223172979 & -0.533465818235 \\ \mathrm{C} & -3.613640001282 & 4.290380602729 & -0.398660251331 \\ \mathrm{C} & -4.287318911747 & 3.109445846390 & -0.084434802631 \\ \mathrm{H} & -4.092828560526 & 1.001931083871 & 0.342344870554 \\ \mathrm{H} & -1.690313609565 & 5.198411628323 & -0.777621286125 \\ \mathrm{H} & -4.167895326223 & 5.215011332723 & -0.536963338986 \\ \mathrm{H} & -5.368624844830 & 3.113508614460 & 0.025168091247 \\ \mathrm{C} & -0.054884182962 & 2.992990158795 & -0.468510810681 \\ \mathrm{C} & 0.523276170081 & 1.784225763034 & -0.325736687052 \\ \mathrm{C} & 2.011344885267 & 1.796517283869 & -0.374654143681 \\ \mathrm{C} & 2.688666023311 & 2.196597254530 & -1.545643704579 \\ \mathrm{C} & 2.798565948512 & 1.478026408836 & 0.751572431100 \\ \mathrm{C} & 4.080561915941 & 2.309825586566 & -1.581723315414 \\ \mathrm{H} & 2.105084047096 & 2.455804737946 & -2.427154656022 \\ \mathrm{C} & 4.185737331465 & 1.600267977168 & 0.716390343706 \\ \mathrm{H} & 2.307357828288 & 1.180834180699 & 1.672942364009 \\ \mathrm{C} & 4.836476290108 & 2.019598889348 & -0.447443453473 \\ \mathrm{H} & 4.569148112887 & 2.635930869765 & -2.495774298318 \\ \mathrm{H} & 4.763435854154 & 1.374174504428 & 1.608859299491 \\ \mathrm{H} & 5.918008809197 & 2.114553480772 & -0.468094557499 \\ \mathrm{Si} & -1.066533121710 & 0.347209133053 & 0.158322432353 \\ \mathrm{H} & 0.512636032935 & 3.919648093415 & -0.606476262267 \\ \mathrm{C} & -0.114816366226 & -0.053202861878 & 1.748403448391 \\ & & & \mathrm{~S}-43 \\ & & & \end{array}$




\begin{tabular}{|c|c|c|c|}
\hline $\mathrm{C}$ & -0.413496353426 & 0.602924060071 & 2.952133442429 \\
\hline $\mathrm{C}$ & 0.911260597227 & -1.008026575536 & 1.771809819675 \\
\hline $\mathrm{C}$ & 0.279864171941 & 0.315899342477 & 4.128891642182 \\
\hline $\mathrm{H}$ & -1.205912175827 & 1.350224010806 & 2.975348984407 \\
\hline $\mathrm{C}$ & 1.599590538222 & -1.318249583901 & 2.945535940623 \\
\hline $\mathrm{H}$ & 1.192080736214 & -1.516053100513 & 0.849446097013 \\
\hline $\mathrm{C}$ & 1.285684888098 & -0.653504231556 & 4.131571308832 \\
\hline $\mathrm{H}$ & 0.030996011138 & 0.841263740441 & 5.047292054978 \\
\hline $\mathrm{H}$ & 2.386365828294 & -2.069912167563 & 2.936306007004 \\
\hline $\mathrm{H}$ & 1.820314826263 & -0.885996755287 & 5.048147219906 \\
\hline $\mathrm{C}$ & -1.708686891288 & -2.871586703906 & -0.042685318612 \\
\hline $\mathrm{C}$ & -2.686583320422 & -1.995569931923 & 0.752862184454 \\
\hline $\mathrm{H}$ & -1.918010776041 & -3.923834355408 & 0.192648609645 \\
\hline $\mathrm{H}$ & -0.698208979865 & -2.669936102662 & 0.334166610195 \\
\hline $\mathrm{O}$ & -0.532602588504 & -0.579243965194 & -1.219215148421 \\
\hline $\mathrm{O}$ & -2.473045710230 & -0.623707266727 & 0.503587928294 \\
\hline $\mathrm{Li}$ & 1.204700539095 & -0.085636966700 & -1.588338046261 \\
\hline $\mathrm{C}$ & 2.078924662374 & -2.884671484815 & -1.226119958561 \\
\hline $\mathrm{O}$ & 2.435493473792 & -1.529026919980 & -1.540037493372 \\
\hline $\mathrm{C}$ & 3.835039097964 & -1.397765369266 & -1.256133936445 \\
\hline $\mathrm{C}$ & 4.003499795675 & -2.139190941624 & 0.063469510070 \\
\hline $\mathrm{C}$ & 3.020539164113 & -3.320377469205 & -0.083107980716 \\
\hline $\mathrm{H}$ & 1.020755193268 & -2.886816196629 & -0.956545408301 \\
\hline $\mathrm{H}$ & 2.220842144358 & -3.499327840471 & -2.123056657652 \\
\hline $\mathrm{H}$ & 4.405939217897 & -1.872545381978 & -2.065786974585 \\
\hline $\mathrm{H}$ & 4.069719175172 & -0.333988594722 & -1.213484700291 \\
\hline $\mathrm{H}$ & 5.032882664415 & -2.462854048581 & 0.233246971699 \\
\hline $\mathrm{H}$ & 3.705216533855 & -1.479482351526 & 0.885032976008 \\
\hline $\mathrm{H}$ & 3.543451928444 & -4.241195764845 & -0.352347461195 \\
\hline $\mathrm{H}$ & 2.469923239210 & -3.509901636681 & 0.842482665830 \\
\hline $\mathrm{C}$ & -2.522617150686 & -2.296088428625 & 2.250124375152 \\
\hline $\mathrm{H}$ & -2.830401438657 & -3.324198873342 & 2.474980167595 \\
\hline $\mathrm{H}$ & -1.485216854015 & -2.165222640636 & 2.572032535634 \\
\hline $\mathrm{H}$ & -3.150165289422 & -1.610367039148 & 2.830246945479 \\
\hline $\mathrm{C}$ & -4.137857709044 & -2.295054583455 & 0.356577223602 \\
\hline $\mathrm{H}$ & -4.813604046686 & -1.686202458678 & 0.967338303415 \\
\hline $\mathrm{H}$ & -4.322389163335 & -2.051181989808 & -0.693740162210 \\
\hline $\mathrm{H}$ & -4.382016881627 & -3.351777376968 & 0.516403147306 \\
\hline $\mathrm{C}$ & -0.518697137684 & -1.510076197792 & -3.402148377546 \\
\hline $\mathrm{H}$ & -0.287514112572 & -0.542005811321 & -3.863717894183 \\
\hline $\mathrm{H}$ & 0.419843786901 & -2.028385169263 & -3.175506599799 \\
\hline $\mathrm{H}$ & -1.064501711193 & -2.113834598354 & -4.134600584471 \\
\hline $\mathrm{C}$ & -2.599314110954 & -0.501847249796 & -2.515808496948 \\
\hline $\mathrm{H}$ & -2.289996770946 & 0.483897368234 & -2.883446871826 \\
\hline $\mathrm{H}$ & -3.146307014835 & -1.012341761696 & -3.316322790362 \\
\hline $\mathrm{H}$ & -3.265633549361 & -0.358074995408 & -1.665011507255 \\
\hline $\mathrm{C}$ & -1.362833002381 & -1.322167404330 & -2.137256252144 \\
\hline $\mathrm{C}$ & -1.725461758204 & -2.717367671206 & -1.565185001718 \\
\hline $\mathrm{H}$ & -2.705455155384 & -3.009360410217 & -1.963033167310 \\
\hline $\mathrm{H}$ & -1.015663398592 & -3.451901798510 & -1.969407680027 \\
\hline
\end{tabular}




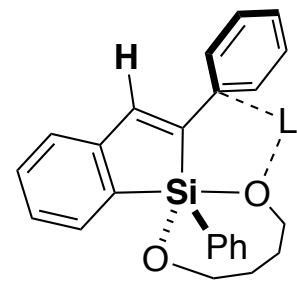

CP7: Free Energy (RM062X): -1764.504318581618 (hartree)

\begin{tabular}{|c|c|c|c|}
\hline $\mathrm{C}$ & 3.926253154273 & 0.487045891699 & 1.052377887012 \\
\hline $\mathrm{C}$ & 2.826324772950 & -0.326920554987 & 0.786100063160 \\
\hline $\mathrm{C}$ & 2.891962635578 & -1.667537038211 & 1.199964016397 \\
\hline $\mathrm{C}$ & 4.009441565519 & -2.189986949937 & 1.854243480618 \\
\hline $\mathrm{C}$ & 5.101848371623 & -1.354802868986 & 2.107027726784 \\
\hline $\mathrm{C}$ & 5.059984178254 & -0.017756660164 & 1.706984796894 \\
\hline $\mathrm{H}$ & 3.909705047030 & 1.531677778208 & 0.741903685630 \\
\hline $\mathrm{H}$ & 4.031998127577 & -3.234436739556 & 2.161680222082 \\
\hline $\mathrm{H}$ & 5.981149259304 & -1.746208664254 & 2.611723303284 \\
\hline $\mathrm{H}$ & 5.911842837503 & 0.629785773558 & 1.901661132956 \\
\hline $\mathrm{C}$ & 1.681960695676 & -2.435847511727 & 0.860318190843 \\
\hline $\mathrm{C}$ & 0.702203971538 & -1.793059209690 & 0.193863553592 \\
\hline $\mathrm{C}$ & -0.542554573990 & -2.527691521776 & -0.128711848121 \\
\hline $\mathrm{C}$ & -0.973795752731 & -2.694804319980 & -1.458867604956 \\
\hline C & -1.357544618254 & -3.049434280189 & 0.892484422131 \\
\hline $\mathrm{C}$ & -2.155768354160 & -3.370011206795 & -1.753414742678 \\
\hline $\mathrm{H}$ & -0.368164005391 & -2.281462858190 & -2.260433921664 \\
\hline $\mathrm{C}$ & -2.548719740404 & -3.719705318001 & 0.599117883113 \\
\hline $\mathrm{H}$ & -1.051131878889 & -2.919325336678 & 1.927441629587 \\
\hline $\mathrm{C}$ & -2.951999100932 & -3.883062274757 & -0.724750281194 \\
\hline $\mathrm{H}$ & -2.460905128330 & -3.492609025293 & -2.788974598807 \\
\hline $\mathrm{H}$ & -3.160063735224 & -4.108347559933 & 1.408541894603 \\
\hline $\mathrm{H}$ & -3.878339057900 & -4.400027230026 & -0.956337891066 \\
\hline $\mathrm{Si}$ & 1.096467040013 & 0.132846076423 & -0.058806455181 \\
\hline $\mathrm{H}$ & 1.615154029192 & -3.490038209077 & 1.141636219124 \\
\hline $\mathrm{C}$ & 0.400758177210 & 1.089452312010 & 1.433770879901 \\
\hline $\mathrm{C}$ & 0.446340857835 & 2.491940813347 & 1.485877246698 \\
\hline $\mathrm{C}$ & -0.144991084316 & 0.426575583590 & 2.545655095727 \\
\hline $\mathrm{C}$ & -0.050721354522 & 3.201166725949 & 2.581424601994 \\
\hline $\mathrm{H}$ & 0.882479434240 & 3.041924442321 & 0.655102474796 \\
\hline $\mathrm{C}$ & -0.642831900231 & 1.124337032203 & 3.648005949463 \\
\hline $\mathrm{H}$ & -0.177615700294 & -0.662312993142 & 2.556612907170 \\
\hline $\mathrm{C}$ & -0.603490444178 & 2.518513085600 & 3.666671828206 \\
\hline $\mathrm{H}$ & -0.001809834091 & 4.287223849966 & 2.590971802932 \\
\hline $\mathrm{H}$ & -1.062857635914 & 0.579805030894 & 4.490099987210 \\
\hline $\mathrm{H}$ & -0.991699730752 & 3.067619207741 & 4.520126528320 \\
\hline $\mathrm{Li}$ & -1.877606577531 & -0.606471335918 & -0.181902675729 \\
\hline $\mathrm{C}$ & -4.659856928825 & -0.287683855087 & 0.667108326127 \\
\hline $\mathrm{O}$ & -3.288754223316 & 0.086039491590 & 0.852974174998 \\
\hline $\mathrm{C}$ & -3.303337428967 & 1.416605499340 & 1.391282900120 \\
\hline $\mathrm{C}$ & -4.403370986533 & 2.132533344745 & 0.604903696067 \\
\hline $\mathrm{C}$ & -5.373558297337 & 0.993788449316 & 0.203674415025 \\
\hline $\mathrm{H}$ & -4.685070971546 & -1.104735492481 & -0.058161301567 \\
\hline $\mathrm{H}$ & -5.053863644664 & -0.646575504238 & 1.625567452892 \\
\hline $\mathrm{H}$ & -3.541578437200 & 1.354651248261 & 2.460736772074 \\
\hline $\mathrm{H}$ & -2.300913527624 & 1.836294064594 & 1.274197238819 \\
\hline $\mathrm{H}$ & -4.887090556638 & 2.905537258399 & 1.205912123044 \\
\hline
\end{tabular}




$\begin{array}{lccc}\mathrm{H} & -3.984050222939 & 2.611883436428 & -0.284668416977 \\ \mathrm{H} & -6.347939613065 & 1.092038939742 & 0.687126394060 \\ \mathrm{H} & -5.536175224623 & 0.982608207164 & -0.876925444318 \\ \mathrm{C} & 2.386382011963 & 1.012215117251 & -2.388600848197 \\ \mathrm{C} & 1.382935547644 & 1.497243068206 & -3.442249405824 \\ \mathrm{C} & -0.000592550759 & 0.847162635690 & -3.391625362891 \\ \mathrm{C} & -0.867555302345 & 1.061277757891 & -2.131308161575 \\ \mathrm{H} & 1.277577445308 & 2.584652070123 & -3.330503639788 \\ \mathrm{H} & 0.078414921238 & -0.236835338459 & -3.552377785674 \\ \mathrm{H} & 1.808831544011 & 1.327646931581 & -4.440672177339 \\ \mathrm{H} & -0.570167547709 & 1.237374902477 & -4.245770466019 \\ \mathrm{O} & 1.835037714295 & 1.264452679525 & -1.105652918071 \\ \mathrm{O} & -0.469909569799 & 0.209096229119 & -1.057933123915 \\ \mathrm{C} & 3.673307238758 & 1.831773856101 & -2.494130298668 \\ \mathrm{H} & 4.394840330630 & 1.490544309423 & -1.744646453713 \\ \mathrm{H} & 4.124905586591 & 1.733875893421 & -3.487847932105 \\ \mathrm{H} & 3.457371846717 & 2.890041719114 & -2.310605949843 \\ \mathrm{C} & 2.718890949468 & -0.472954183959 & -2.585134171375 \\ \mathrm{H} & 3.117418989690 & -0.645298765905 & -3.591137933068 \\ \mathrm{H} & 3.467528220733 & -0.795029931138 & -1.853506780037 \\ \mathrm{H} & 1.831771255325 & -1.106075855577 & -2.463385994927 \\ \mathrm{C} & -0.900241495850 & 2.530179515916 & -1.703897247636 \\ \mathrm{H} & -1.267623879136 & 3.159889841292 & -2.523752271585 \\ \mathrm{H} & -1.572157348344 & 2.651816147240 & -0.845671276512 \\ \mathrm{H} & 0.092527871422 & 2.874002265503 & -1.412475377429 \\ \mathrm{C} & -2.298530543047 & 0.631525393129 & -2.500025156730 \\ \mathrm{H} & -2.974806940056 & 0.748074552238 & -1.641562327741 \\ \mathrm{H} & -2.702586424476 & 1.247928869853 & -3.309366667449 \\ \mathrm{H} & -2.315207756291 & -0.415658704060 & -2.827420993092 \\ * * * * * * * * * * * * * * * * * * * * * * * * * * * * * * * * * * * * * * * * * * * * * * * * * * * * * * * * * * * * * * * * * * * * * * * * * * * * * * * * * *\end{array}$

- Table 3b

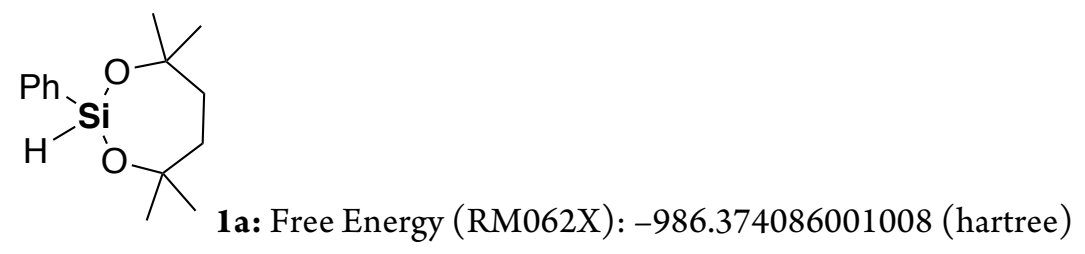

$\begin{array}{lccc}* * * * * * * * * * * * * * * * * * * * * * * * * * * * * * * * * * * * * * * * * * * * * * * * * * * * * * * * * * * * * * * * * * * * * * * * * * * * * * * * * * * * * * \\ \mathrm{Si} & -0.107728722897 & -0.235397427036 & -1.051102394753 \\ \mathrm{H} & -0.059129922406 & -0.498219904927 & -2.502630006120 \\ \mathrm{C} & -1.861433697929 & -0.324805719647 & -0.421609383699 \\ \mathrm{C} & -2.215533406184 & -1.132741424542 & 0.665309090421 \\ \mathrm{C} & -2.859156408409 & 0.446555803315 & -1.031293860030 \\ \mathrm{C} & -3.526662841680 & -1.166749258643 & 1.132328632568 \\ \mathrm{H} & -1.454590462806 & -1.736947709629 & 1.148685215731 \\ \mathrm{C} & -4.170779619380 & 0.418851548298 & -0.566121387697 \\ \mathrm{H} & -2.613803898345 & 1.080714321600 & -1.878971161773 \\ \mathrm{C} & -4.504804345963 & -0.389338063277 & 0.517662364023 \\ \mathrm{H} & -3.786186104359 & -1.798086462159 & 1.974431978136 \\ \mathrm{H} & -4.930554742901 & 1.023710380977 & -1.047451714105 \\ \mathrm{H} & -5.525624952724 & -0.414046148804 & 0.881353503460 \\ \mathrm{C} & 1.145637933665 & 1.816529261201 & 0.307861263928 \\ \mathrm{C} & 2.600719049390 & -0.274336690045 & 1.006013724903 \\ \mathrm{C} & 2.167666576176 & -1.367552546273 & 0.014869960483 \\ & & \mathrm{~S}-46 & \end{array}$




$\begin{array}{lccc}\mathrm{H} & 3.644529246757 & -0.479808064901 & 1.265243416725 \\ \mathrm{H} & 2.024390298372 & -0.418850884589 & 1.925395002674 \\ \mathrm{O} & 0.581216185270 & 1.258757059905 & -0.893961464355 \\ \mathrm{O} & 0.737228353580 & -1.331387986510 & -0.151899563964 \\ \mathrm{C} & 2.528098713505 & 1.192637395296 & 0.561528360752 \\ \mathrm{H} & 3.126126444392 & 1.341839625406 & -0.343183337774 \\ \mathrm{H} & 3.006097742656 & 1.789330356720 & 1.345434292191 \\ \mathrm{C} & 1.313240621776 & 3.305112117809 & 0.021492593480 \\ \mathrm{H} & 1.986301971644 & 3.450908133344 & -0.826176706200 \\ \mathrm{H} & 0.344565044903 & 3.745136825277 & -0.223296620329 \\ \mathrm{H} & 1.724600380306 & 3.821916676157 & 0.891121993272 \\ \mathrm{C} & 0.196825703010 & 1.618572925337 & 1.489824441888 \\ \mathrm{H} & -0.765819929268 & 2.090041014894 & 1.277751893576 \\ \mathrm{H} & 0.019073451603 & 0.561353609588 & 1.698122412362 \\ \mathrm{H} & 0.621307041168 & 2.075330058154 & 2.387215681337 \\ \mathrm{C} & 2.860482321989 & -1.229287182784 & -1.340192209518 \\ \mathrm{H} & 2.561011936841 & -2.050502397942 & -1.995449662470 \\ \mathrm{H} & 2.609433267789 & -0.285825388199 & -1.830441382025 \\ \mathrm{H} & 3.944965380240 & -1.264769011686 & -1.211983881483 \\ \mathrm{C} & 2.469581360360 & -2.735541044993 & 0.617231497140 \\ \mathrm{H} & 3.544520560187 & -2.866525629898 & 0.758113680352 \\ \mathrm{H} & 1.972915027645 & -2.837393830640 & 1.584688544012 \\ \mathrm{H} & 2.103856442028 & -3.521269336155 & -0.046530807123 \\ * * * * * * * * * * * * * * * * * * * * * * * * * * * * * * * * * * * * * * * * * * * * * * * * * * * * * * * * * * * * * * * * * * * * * * * * * * * * * * * * * * * * * * * *\end{array}$

$$
\text { 1b: Free Energy (RM062X): -947.092874809139 (hartree) }
$$

$\begin{array}{lccc}* * * * * * * * * * * * * * * * * * * * * * * * * * * * * * * * * * * * * * * * * * * * * * * * * * * * * * * * * * * * * * * * * * * * * * * * * * * * * * * * * * * * * \\ \mathrm{Si} & 0.009699558117 & -0.089998677264 & -1.306196701131 \\ \mathrm{H} & 0.072156657774 & -0.041716740975 & -2.777100896321 \\ \mathrm{C} & 1.714603572397 & -0.196192151830 & -0.554597530019 \\ \mathrm{C} & 2.727250283200 & 0.681371406161 & -0.964601883069 \\ \mathrm{C} & 1.997340094099 & -1.107824764175 & 0.469623635014 \\ \mathrm{C} & 3.981925912243 & 0.656355285668 & -0.363910522738 \\ \mathrm{H} & 2.537602624036 & 1.396385256894 & -1.760534862232 \\ \mathrm{C} & 3.251987510811 & -1.137678999656 & 1.073521476793 \\ \mathrm{H} & 1.227449624687 & -1.801828299273 & 0.793094479100 \\ \mathrm{C} & 4.243785750832 & -0.253682884104 & 0.658039268173 \\ \mathrm{H} & 4.754649310318 & 1.342411886627 & -0.690901803413 \\ \mathrm{H} & 3.456941192000 & -1.849747795445 & 1.864655923178 \\ \mathrm{H} & 5.220881466909 & -0.275300026159 & 1.126789954902 \\ \mathrm{C} & -2.020782839737 & -1.139188848390 & 0.106042186485 \\ \mathrm{C} & -1.692003366533 & 0.001507093285 & 1.089382110786 \\ \mathrm{C} & -1.383797875792 & 1.395511288313 & 0.485950671757 \\ \mathrm{H} & -2.513602699178 & 0.104805928018 & 1.804030188190 \\ \mathrm{H} & -0.817787754210 & -0.332961910651 & 1.660924219513 \\ \mathrm{O} & -0.874350861039 & -1.372311943023 & -0.741895649602 \\ \mathrm{O} & -0.812308399312 & 1.268437101437 & -0.832785877901 \\ \mathrm{C} & -2.229768236262 & -2.426246846196 & 0.893579768321 \\ \mathrm{H} & -3.109356049139 & -2.343958749210 & 1.536137929660 \\ \mathrm{H} & -2.375773172805 & -3.259341835879 & 0.203308886968 \\ \mathrm{H} & -1.357086210950 & -2.637044944730 & 1.515416962340 \\ \mathrm{C} & -3.232182413380 & -0.864826869711 & -0.782460436484 \\ & & & \end{array}$




$\begin{array}{lccc}\mathrm{H} & -4.113346707382 & -0.639222169175 & -0.176659952409 \\ \mathrm{H} & -3.044815728283 & -0.027345257869 & -1.455825580284 \\ \mathrm{H} & -3.442142194920 & -1.750280370230 & -1.386231354563 \\ \mathrm{C} & -2.639046161320 & 2.249434200880 & 0.337473792414 \\ \mathrm{H} & -2.377489106429 & 3.196729575672 & -0.138251767711 \\ \mathrm{H} & -3.395827337588 & 1.753964013245 & -0.269387842121 \\ \mathrm{H} & -3.064977929827 & 2.459769774532 & 1.321319602737 \\ \mathrm{C} & -0.374521885321 & 2.122052505514 & 1.373131451620 \\ \mathrm{H} & 0.565948506657 & 1.566953085148 & 1.415602141788 \\ \mathrm{H} & -0.175831723055 & 3.118448211639 & 0.972664967799 \\ \mathrm{H} & -0.765634411617 & 2.222412470915 & 2.388898042462 \\ * * * * * * * * * * * * * * * * * * * * * * * * * * * * * * * * * * * * * * * * * * * * * * * * * * * * * * * * * * * * * * * * * * * * * * * * * * * * * * * * * * * * * * * * * *\end{array}$

- Table 3c (THF and the exact structure of the ligand are omitted for clarity)

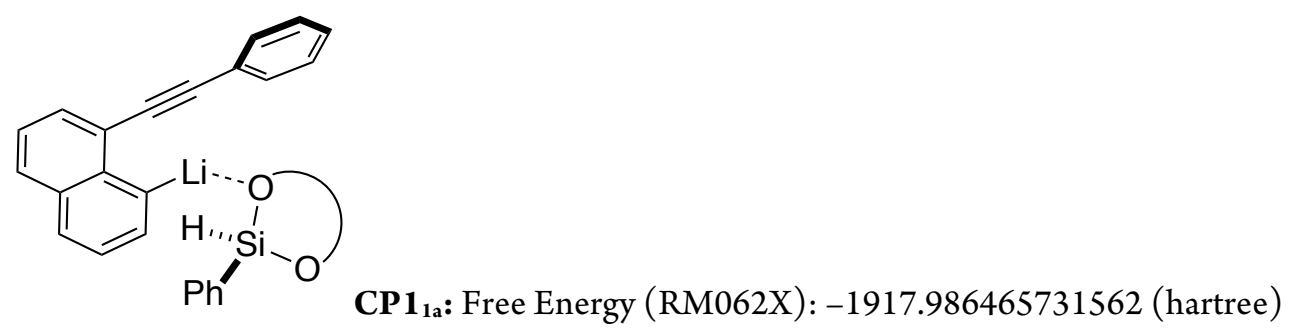

C

C

C

$\mathrm{C}$

$\mathrm{H}$

$\mathrm{H}$

C

C

$\mathrm{H}$

C

C

3.421725485437

$\mathrm{H} \quad 5.349835649911$

C $\quad 1.567390207905$

C $\quad 0.660747405310$

C $\quad-0.440344240838$

C $\quad-0.473847007643$

C $\quad-1.504770972381$

C $\quad-1.559389373815$

$\mathrm{H} \quad 0.344928493979$

C $\quad-2.588672794936$

$\mathrm{H} \quad-1.467683263894$

C $\quad-2.622142410755$

$\mathrm{H} \quad-1.575426864191$

$\mathrm{H} \quad-3.407593186363$

$\mathrm{H} \quad-3.469191546425$

$\mathrm{Li} \quad 0.491126730304$

C $\quad 1.900490666653$
$-2.951045415943$

$-2.773613745889$

$-1.677455682335$

$-0.790187729855$

$-0.946436907575$

$-2.043261810424$

$-2.159151467200$

$-3.791356045573$

$-3.457458392984$

$-1.469396157075$

0.282879413014

$-2.250326233482$

0.461486483373

$-0.423509193761$

1.280593013176

$-0.273238255658$

1.193884476405

2.006405307314

2.933493127388

4.069060811338

2.701204964893

4.939153627229

4.264475147023

3.573961319864

1.834659663948

4.692168410452

5.810934962064

3.381103919747

5.370683720302

0.591368549879

1.064733455490

S-48
$-1.416577949154$

$-0.391666769762$

0.499786095768

0.297715012881

$-0.766980198454$

$-1.586179143604$

1.705286969778

$-2.099156855459$

$-0.244828910234$

1.579439484253

1.243793532716

$-2.420178147449$

2.288682791236

2.454923654881

2.986879565014

3.278101471609

1.135378309335

1.135314199639

1.182118462625

0.358559556798

2.067760236194

0.404432764976

$-0.325968406902$

2.108864294694

2.718990306058

1.274198456140

$-0.242658709633$

2.795344831680

1.305796080134

$-0.977256845546$

$-3.399155737739$ 


\begin{tabular}{|c|c|c|c|}
\hline $\mathrm{O}$ & 1.318187055440 & 1.812692403612 & -2.317433512570 \\
\hline $\mathrm{C}$ & 2.297016523765 & 2.789841845055 & -1.946777433676 \\
\hline $\mathrm{C}$ & 3.625698563520 & 2.041293433218 & -1.993006479666 \\
\hline $\mathrm{C}$ & 3.417711500036 & 1.029738388547 & -3.139775102756 \\
\hline $\mathrm{H}$ & 1.446663588395 & 0.072132195978 & -3.394763613783 \\
\hline $\mathrm{H}$ & 1.658891329323 & 1.576741153183 & -4.339419243965 \\
\hline $\mathrm{H}$ & 2.267102024922 & 3.614225480570 & -2.673109715925 \\
\hline $\mathrm{H}$ & 2.042554384430 & 3.163069965218 & -0.954783517550 \\
\hline $\mathrm{H}$ & 4.469199363062 & 2.714572679785 & -2.164618954157 \\
\hline $\mathrm{H}$ & 3.783121738636 & 1.516381621782 & -1.046505526300 \\
\hline $\mathrm{H}$ & 3.968148071336 & 1.317107062939 & -4.039554333616 \\
\hline $\mathrm{H}$ & 3.736430572894 & 0.028394749719 & -2.840456430229 \\
\hline $\mathrm{Si}$ & -1.458957426534 & -1.591604178202 & -0.157744172448 \\
\hline $\mathrm{H}$ & -0.882925153840 & -2.568462405523 & -1.105376484062 \\
\hline $\mathrm{C}$ & -0.650019377712 & -1.577611791399 & 1.515151133721 \\
\hline $\mathrm{C}$ & -1.267879035155 & -0.868388348293 & 2.556183444374 \\
\hline $\mathrm{C}$ & 0.507283773882 & -2.311590177939 & 1.805550386313 \\
\hline $\mathrm{C}$ & -0.724572993117 & -0.854386165177 & 3.839923032888 \\
\hline $\mathrm{H}$ & -2.190131490636 & -0.324622827511 & 2.359914147903 \\
\hline $\mathrm{C}$ & 1.051254828423 & -2.310628448661 & 3.090034333375 \\
\hline $\mathrm{H}$ & 1.006136459500 & -2.870786928969 & 1.016953129802 \\
\hline $\mathrm{C}$ & 0.442359300415 & -1.573688812534 & 4.105675319571 \\
\hline $\mathrm{H}$ & -1.210037086323 & -0.290311229258 & 4.632029692846 \\
\hline $\mathrm{H}$ & 1.959822220862 & -2.871727079228 & 3.292246291042 \\
\hline $\mathrm{H}$ & 0.872396790783 & -1.563021314743 & 5.103438639435 \\
\hline $\mathrm{C}$ & -3.715880694911 & -0.034666949925 & -1.769446890411 \\
\hline $\mathrm{C}$ & -2.269318586225 & 0.474315674736 & -1.890751265091 \\
\hline $\mathrm{H}$ & -4.294801765056 & 0.537328485987 & -2.502554471923 \\
\hline $\mathrm{H}$ & -4.091295548368 & 0.259063397513 & -0.781915196311 \\
\hline $\mathrm{O}$ & -1.410417837729 & -0.048542498854 & -0.840173130346 \\
\hline $\mathrm{O}$ & -3.030645262744 & -1.985005948013 & 0.197426083127 \\
\hline $\mathrm{C}$ & -1.645951436125 & 0.111503385953 & -3.240189799114 \\
\hline $\mathrm{H}$ & -2.331121469865 & 0.357804981058 & -4.058469356506 \\
\hline $\mathrm{H}$ & -0.724503888537 & 0.681890034821 & -3.388827150102 \\
\hline $\mathrm{H}$ & -1.399916180584 & -0.953111289611 & -3.303080832440 \\
\hline $\mathrm{C}$ & -2.269196791779 & 1.989581111894 & -1.708833725636 \\
\hline $\mathrm{H}$ & -1.254411339219 & 2.389287250039 & -1.805625049245 \\
\hline $\mathrm{H}$ & -2.890223996937 & 2.463045616466 & -2.476572745585 \\
\hline $\mathrm{H}$ & -2.657706789873 & 2.260489624622 & -0.720800899514 \\
\hline $\mathrm{C}$ & -3.942459701200 & -3.850935905942 & -1.022965063330 \\
\hline $\mathrm{H}$ & -4.030851717872 & -4.434355267796 & -0.101131441818 \\
\hline $\mathrm{H}$ & -4.730262622797 & -4.168694839728 & -1.714390893278 \\
\hline $\mathrm{H}$ & -2.972858815762 & -4.071236936433 & -1.481713091156 \\
\hline $\mathrm{C}$ & -5.388161354957 & -2.086034305182 & 0.002978687580 \\
\hline $\mathrm{H}$ & -6.233671067150 & -2.403929196068 & -0.615536936716 \\
\hline $\mathrm{H}$ & -5.415802059107 & -2.641982707736 & 0.945231431782 \\
\hline $\mathrm{H}$ & -5.503007378037 & -1.022506768792 & 0.232408281413 \\
\hline $\mathrm{C}$ & -4.068935394790 & -2.359796271906 & -0.715045484292 \\
\hline $\mathrm{C}$ & -3.948103139061 & -1.536223129668 & -2.005961513248 \\
\hline $\mathrm{H}$ & -3.137969794311 & -1.958350144392 & -2.615813004616 \\
\hline $\mathrm{H}$ & -4.862667559766 & -1.686576810667 & -2.590495926366 \\
\hline
\end{tabular}




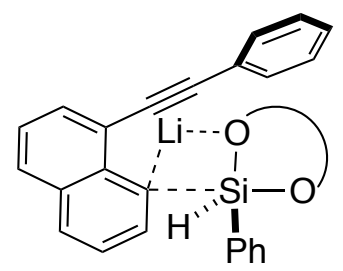

CP2 2a: Free Energy (RM062X): -1917.973720836187 (hartree)

\begin{tabular}{|c|c|c|c|}
\hline $\mathrm{C}$ & 2.828329928188 & -2.751567830613 & -1.835621805389 \\
\hline $\mathrm{C}$ & 3.827000628625 & -2.489307578312 & -0.934369064354 \\
\hline $\mathrm{C}$ & 3.619203508150 & -1.520729075404 & 0.085861120734 \\
\hline $\mathrm{C}$ & 2.360861312667 & -0.843992178553 & 0.161082055590 \\
\hline $\mathrm{C}$ & 1.253092152249 & -1.203829293019 & -0.695869458307 \\
\hline $\mathrm{C}$ & 1.564864993772 & -2.119390801295 & -1.688918771085 \\
\hline $\mathrm{H}$ & 5.595278011701 & -1.774818462827 & 0.928281772537 \\
\hline $\mathrm{H}$ & 2.985274696649 & -3.471121023419 & -2.635649410365 \\
\hline $\mathrm{H}$ & 4.786745543913 & -2.998157235377 & -0.989392970213 \\
\hline $\mathrm{C}$ & 4.659176573407 & -1.226512577324 & 1.009205081719 \\
\hline $\mathrm{C}$ & 2.260526223318 & 0.199031560453 & 1.150611087720 \\
\hline $\mathrm{H}$ & 0.779866664295 & -2.438620620283 & -2.376573559697 \\
\hline $\mathrm{C}$ & 3.293323315889 & 0.466203043354 & 2.033801246974 \\
\hline $\mathrm{C}$ & 4.496481283593 & -0.267956777216 & 1.979502140344 \\
\hline $\mathrm{H}$ & 3.175337336470 & 1.264436699476 & 2.760732368903 \\
\hline $\mathrm{H}$ & 5.292856178956 & -0.050793144374 & 2.684495762302 \\
\hline $\mathrm{C}$ & 1.125966303084 & 1.081173877514 & 1.173692048548 \\
\hline $\mathrm{C}$ & 0.248184304061 & 1.921517581568 & 1.150608546427 \\
\hline $\mathrm{C}$ & -0.781875112181 & 2.920642769734 & 1.070422638344 \\
\hline $\mathrm{C}$ & -0.577873454954 & 4.063810278408 & 0.282311320816 \\
\hline $\mathrm{C}$ & -1.998009076608 & 2.7587455333538 & 1.749882396349 \\
\hline $\mathrm{C}$ & -1.581417679659 & 5.020832611186 & 0.164868967127 \\
\hline $\mathrm{H}$ & 0.363323621041 & 4.186732708618 & -0.246509305755 \\
\hline $\mathrm{C}$ & -2.997660886295 & 3.720305172600 & 1.629393409991 \\
\hline $\mathrm{H}$ & -2.153182875427 & 1.878810506628 & 2.364596897493 \\
\hline $\mathrm{C}$ & -2.793990514906 & 4.849618883050 & 0.834706145822 \\
\hline $\mathrm{H}$ & -1.418604694824 & 5.898943957957 & -0.452532701234 \\
\hline $\mathrm{H}$ & -3.938315680825 & 3.585823155907 & 2.154546317392 \\
\hline $\mathrm{H}$ & -3.577352070987 & 5.595376599826 & 0.739527654485 \\
\hline $\mathrm{Li}$ & 0.449551405461 & 0.788701358402 & -1.032718527346 \\
\hline $\mathrm{C}$ & 2.351712767223 & 1.274378585501 & -3.123880037899 \\
\hline $\mathrm{O}$ & 1.776294432642 & 1.842627229437 & -1.939051190135 \\
\hline $\mathrm{C}$ & 2.820154752649 & 2.623674145801 & -1.340775149171 \\
\hline $\mathrm{C}$ & 4.102614633098 & 1.802418586001 & -1.532258831035 \\
\hline $\mathrm{C}$ & 3.769109881462 & 0.862806773233 & -2.716423686226 \\
\hline $\mathrm{H}$ & 1.724082069517 & 0.436453900259 & -3.436003049828 \\
\hline $\mathrm{H}$ & 2.355939323275 & 2.041547410719 & -3.909173475570 \\
\hline $\mathrm{H}$ & 2.874677714216 & 3.584486417074 & -1.869255870081 \\
\hline $\mathrm{H}$ & 2.552323422397 & 2.797017912716 & -0.297180521141 \\
\hline $\mathrm{H}$ & 4.955110242930 & 2.453113396761 & -1.741651188814 \\
\hline $\mathrm{H}$ & 4.327932815803 & 1.223289423978 & -0.632204863019 \\
\hline $\mathrm{H}$ & 4.468527407376 & 0.969190418452 & -3.549159631580 \\
\hline $\mathrm{H}$ & 3.775552345062 & -0.180594125149 & -2.391905992109 \\
\hline $\mathrm{Si}$ & -1.029398621417 & -1.418969543912 & -0.175389580240 \\
\hline $\mathrm{H}$ & -0.916252199416 & -2.512865773714 & -1.169890934107 \\
\hline $\mathrm{C}$ & -0.432185793346 & -1.653469405402 & 1.616365522183 \\
\hline $\mathrm{C}$ & -1.043522409747 & -0.963265954130 & 2.672792948754 \\
\hline
\end{tabular}




\begin{tabular}{|c|c|c|c|}
\hline $\mathrm{C}$ & 0.565055793654 & -2.581542928208 & 1.947651819418 \\
\hline $\mathrm{C}$ & -0.640701959987 & -1.141057190858 & 3.995858746214 \\
\hline $\mathrm{H}$ & -1.855973591138 & -0.275353189919 & 2.452899199885 \\
\hline $\mathrm{C}$ & 0.954125452417 & -2.795816804415 & 3.271595133175 \\
\hline $\mathrm{H}$ & 1.063209193159 & -3.143455577346 & 1.158937486915 \\
\hline $\mathrm{C}$ & 0.364890054040 & -2.060885596347 & 4.300131207045 \\
\hline $\mathrm{H}$ & -1.117483178181 & -0.572122110058 & 4.790124345369 \\
\hline $\mathrm{H}$ & 1.726816255294 & -3.526525229374 & 3.496493548803 \\
\hline $\mathrm{H}$ & 0.678896984598 & -2.209320476535 & 5.329746215057 \\
\hline $\mathrm{C}$ & -3.645823453010 & -0.039996457251 & -1.705775775383 \\
\hline $\mathrm{C}$ & -2.217254118286 & 0.475440039241 & -1.947367606338 \\
\hline $\mathrm{H}$ & -4.268530347415 & 0.442930321805 & -2.468489473606 \\
\hline $\mathrm{H}$ & -3.979062976573 & 0.350125221091 & -0.736777528766 \\
\hline $\mathrm{O}$ & -1.296247674516 & 0.132880094562 & -0.890988390138 \\
\hline $\mathrm{O}$ & -2.659303006598 & -1.719307597387 & 0.336449334278 \\
\hline $\mathrm{C}$ & -1.651966723158 & -0.044437168030 & -3.274903058649 \\
\hline $\mathrm{H}$ & -2.376182467619 & 0.095598800483 & -4.085128246781 \\
\hline $\mathrm{H}$ & -0.740606683072 & 0.501598542649 & -3.542803979052 \\
\hline $\mathrm{H}$ & -1.400094813808 & -1.107476480845 & -3.217264132115 \\
\hline $\mathrm{C}$ & -2.268299992453 & 2.003891445070 & -1.977883579913 \\
\hline $\mathrm{H}$ & -1.267463572886 & 2.430187828405 & -2.113960563679 \\
\hline $\mathrm{H}$ & -2.892963898131 & 2.346817831050 & -2.809503381428 \\
\hline $\mathrm{H}$ & -2.680947399935 & 2.394167646492 & -1.041432985781 \\
\hline $\mathrm{C}$ & -3.601705522523 & -3.751554186433 & -0.571865843598 \\
\hline $\mathrm{H}$ & -3.543293863238 & -4.238590384042 & 0.407515006819 \\
\hline $\mathrm{H}$ & -4.457522816706 & -4.167016799983 & -1.116990281129 \\
\hline $\mathrm{H}$ & -2.690631024807 & -3.988306499264 & -1.129883730467 \\
\hline $\mathrm{C}$ & -5.005788251211 & -1.957973460046 & 0.441978687440 \\
\hline $\mathrm{H}$ & -5.897303986158 & -2.382757465862 & -0.033047823256 \\
\hline $\mathrm{H}$ & -4.890155448371 & -2.407515807036 & 1.433607262976 \\
\hline $\mathrm{H}$ & -5.160023611784 & -0.882260894066 & 0.575114118447 \\
\hline $\mathrm{C}$ & -3.752454988550 & -2.236488380949 & -0.393561098038 \\
\hline $\mathrm{C}$ & -3.862192100200 & -1.556363571539 & -1.768185488924 \\
\hline $\mathrm{H}$ & -3.141419157134 & -2.017418876073 & -2.455232677404 \\
\hline $\mathrm{H}$ & -4.853075194015 & -1.773887661860 & -2.186063070918 \\
\hline
\end{tabular}

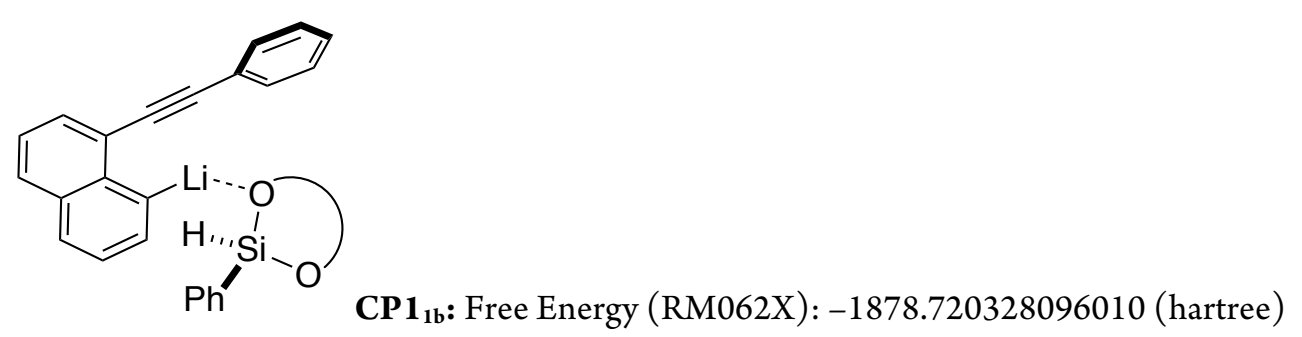

$\begin{array}{lllc}\mathrm{C} & 4.563573514954 & -0.195717015673 & 0.480545668287 \\ \mathrm{C} & 4.939624504799 & -0.786304345412 & -0.701527547541 \\ \mathrm{C} & 3.954312164390 & -1.410072231302 & -1.512280647400 \\ \mathrm{C} & 2.593110783327 & -1.396530985784 & -1.067786364387 \\ \mathrm{C} & 2.157024705972 & -0.754226281266 & 0.146714886926 \\ \mathrm{C} & 3.198447506462 & -0.187748654675 & 0.876181035350 \\ \mathrm{H} & 5.346922529062 & -2.030291334537 & -3.050415611718 \\ \mathrm{H} & 5.313359725214 & 0.277266247649 & 1.112693820460\end{array}$




\begin{tabular}{|c|c|}
\hline $\mathrm{H}$ & 5.977301744630 \\
\hline $\mathrm{C}$ & 4.304961918903 \\
\hline $\mathrm{C}$ & 1.640735285865 \\
\hline $\mathrm{H}$ & 2.984220907149 \\
\hline $\mathrm{C}$ & 2.008575423594 \\
\hline $\mathrm{C}$ & 3.355518200669 \\
\hline $\mathrm{H}$ & 1.258227768444 \\
\hline $\mathrm{H}$ & 3.633381034804 \\
\hline $\mathrm{C}$ & 0.266397470518 \\
\hline $\mathrm{C}$ & -0.906291859983 \\
\hline $\mathrm{C}$ & -2.301449748643 \\
\hline $\mathrm{C}$ & -2.746992189400 \\
\hline $\mathrm{C}$ & -3.228517273272 \\
\hline $\mathrm{C}$ & -4.099583710269 \\
\hline $\mathrm{H}$ & -2.026015984675 \\
\hline $\mathrm{C}$ & -4.580145222256 \\
\hline $\mathrm{H}$ & -2.877196732375 \\
\hline $\mathrm{C}$ & -5.018134610214 \\
\hline $\mathrm{H}$ & -4.437439586858 \\
\hline $\mathrm{H}$ & -5.291969411642 \\
\hline $\mathrm{H}$ & -6.072200263372 \\
\hline $\mathrm{Li}$ & 0.136014938375 \\
\hline $\mathrm{C}$ & 0.577586910947 \\
\hline $\mathrm{O}$ & 0.004454986629 \\
\hline $\mathrm{C}$ & 0.694617405312 \\
\hline $\mathrm{C}$ & 1.990889506482 \\
\hline $\mathrm{C}$ & 1.552479162324 \\
\hline $\mathrm{H}$ & 1.097599659926 \\
\hline $\mathrm{H}$ & -0.235451656113 \\
\hline $\mathrm{H}$ & 0.057662692261 \\
\hline $\mathrm{H}$ & 0.847317079777 \\
\hline $\mathrm{H}$ & 2.385220766165 \\
\hline $\mathrm{H}$ & 2.740741814707 \\
\hline $\mathrm{H}$ & 1.035839772495 \\
\hline $\mathrm{H}$ & 2.381694847544 \\
\hline $\mathrm{Si}$ & -0.391555396779 \\
\hline $\mathrm{C}$ & -2.808110787924 \\
\hline $\mathrm{C}$ & -2.087597831041 \\
\hline $\mathrm{O}$ & -0.965230740285 \\
\hline $\mathrm{O}$ & -1.718815812223 \\
\hline $\mathrm{C}$ & -1.560577007945 \\
\hline $\mathrm{H}$ & -2.391189248033 \\
\hline $\mathrm{H}$ & -0.867202139950 \\
\hline $\mathrm{H}$ & -1.026541561338 \\
\hline $\mathrm{C}$ & -2.680805050060 \\
\hline $\mathrm{H}$ & -1.926642849020 \\
\hline $\mathrm{H}$ & -3.514048394965 \\
\hline $\mathrm{H}$ & -3.050246249789 \\
\hline $\mathrm{C}$ & -2.449060451630 \\
\hline $\mathrm{H}$ & -2.306363570646 \\
\hline $\mathrm{H}$ & -3.256603389066 \\
\hline $\mathrm{H}$ & -1.524951694866 \\
\hline $\mathrm{C}$ & -3.993788712728 \\
\hline $\mathrm{H}$ & -4.876133059579 \\
\hline $\mathrm{H}$ & -3.747798305023 \\
\hline $\mathrm{H}$ & -4.234724752732 \\
\hline
\end{tabular}

$-0.790479038783$

$-2.043640740859$

$-2.081159356824$

0.314603097129

$-2.692808054380$

$-2.6666634917685$

$-3.200018627448$

$-3.147359883919$

$-2.157565287962$

$-2.269504395623$

$-2.371533815390$

$-3.302296748021$

$-1.526461885634$

$-3.378675022145$

$-3.952712044715$

$-1.610670452612$

$-0.801916297362$

$-2.533206576060$

$-4.097332184660$

$-0.950429728127$

$-2.593881825349$

$-0.941783937886$

$-1.442287190179$

$-2.120992594544$

$-3.368445635666$

$-3.267012608200$

$-2.431051809924$

$-0.545288180772$

$-1.142353075871$

$-4.183797423810$

$-3.477908687926$

$-4.247866213499$

$-2.726716808781$

$-3.057882981653$

$-1.923689635025$

1.560996782594

2.785685149329

1.149818127432

0.670684635624

2.286097314995

2.111214479248

2.489468428302

1.577223717094

2.963129196008

$-0.073839837998$

$-0.567602075311$

0.229336580755

$-0.794276562030$

4.168922756726

4.862176672405

4.549353220702

4.163946455454

2.921091731155

3.292644772423

3.623084919902

1.951191374988
$-1.028626399351$

$-2.736199485461$

$-1.900302190984$

1.823599360013

$-3.086634884648$

$-3.510979891590$

$-3.686190732323$

$-4.444229122774$

$-1.484337790992$

$-1.183395473550$

$-0.859601765221$

0.091698743134

$-1.489048910485$

0.412046198124

0.578279319690

$-1.165195612039$

$-2.219128209475$

$-0.213427489707$

1.152398213680

$-1.652361601744$

0.039676357823

0.689082854184

3.365155699904

2.227809192882

2.001706411763

2.794325846684

4.000012220002

3.003083982430

4.032352772064

2.366122485907

0.924331907314

3.070540373822

2.207833144189

4.734597970597

4.498608895501

$-0.874954817388$

$-0.759816108055$

1.231970267973

0.433506317902

$-1.556075901262$

2.297525144836

2.902663882945

2.957207115409

1.875576420988

1.923022491201

2.543138636256

2.565787048689

1.188461337087

$-0.210929105523$

$-1.046428072627$

0.423707042371

0.369573090264

$-1.712877125600$

$-1.181173424993$

$-2.515578123885$

$-2.159856743121$

S-52 


$\begin{array}{lrlr}\mathrm{C} & 0.836678502882 & 2.850928111329 & -0.314984315450 \\ \mathrm{C} & 1.096476415876 & 3.961164136262 & -1.134034782486 \\ \mathrm{C} & 1.526383156002 & 2.746629033249 & 0.900176392359 \\ \mathrm{C} & 2.018795473086 & 4.935349680765 & -0.752574517468 \\ \mathrm{H} & 0.566414531576 & 4.073917531176 & -2.078234808662 \\ \mathrm{C} & 2.447900497032 & 3.717874247470 & 1.290217811258 \\ \mathrm{H} & 1.340002749713 & 1.892496776755 & 1.546617628392 \\ \mathrm{C} & 2.695782586224 & 4.813268608039 & 0.461965588466 \\ \mathrm{H} & 2.207673469583 & 5.788147668671 & -1.398276552788 \\ \mathrm{H} & 2.974400614550 & 3.619266878786 & 2.235565380831 \\ \mathrm{H} & 3.414676916712 & 5.570413586088 & 0.761956016496 \\ \mathrm{H} & 0.153052661253 & 0.646665121989 & -1.885794761552 \\ \mathrm{C} & -3.178098272620 & 1.747486660532 & 0.318839788581 \\ \mathrm{H} & -3.955316398767 & 2.175965136208 & 0.963576542386 \\ \mathrm{H} & -3.634910913913 & 0.901234215894 & -0.208220223249 \\ * * * * * * * * * * * * * * * * * * * * * * * * * * * * * * * * * * * * * * * * * * * * * * * * * * * * * * * * * * * * * * * * * * * * * * * * * * * * * * * * * * * * * * * * * * *\end{array}$

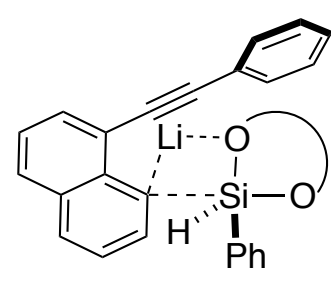

CP2 1 : Free Energy (RM062X): -1878.715652817094 (hartree)

$\begin{array}{llcc}\mathrm{C} & 3.958333063658 & -0.003155750801 & 0.927137632492 \\ \mathrm{C} & 4.478068823176 & -0.821848995569 & -0.042246615883 \\ \mathrm{C} & 3.615277258177 & -1.411931788627 & -1.006936362837 \\ \mathrm{C} & 2.222204911536 & -1.106161058391 & -0.966576460305 \\ \mathrm{C} & 1.696473774756 & -0.110915201086 & -0.069377537375 \\ \mathrm{C} & 2.582446852164 & 0.347278274787 & 0.893150885366 \\ \mathrm{H} & 5.187993947882 & -2.524347529375 & -1.991896294817 \\ \mathrm{H} & 4.603058051385 & 0.419001513325 & 1.693568128969 \\ \mathrm{H} & 5.539501987227 & -1.056310068739 & -0.072287381384 \\ \mathrm{C} & 4.118517598873 & -2.328402468166 & -1.969558177721 \\ \mathrm{C} & 1.364399291575 & -1.872117910928 & -1.826461103879 \\ \mathrm{H} & 2.237083326876 & 1.068163415510 & 1.629816531696 \\ \mathrm{C} & 1.880940127835 & -2.767324903514 & -2.746923316854 \\ \mathrm{C} & 3.274309819973 & -2.971355512647 & -2.842682099908 \\ \mathrm{H} & 1.202756969469 & -3.340321043470 & -3.371973476335 \\ \mathrm{H} & 3.665185397704 & -3.669366886517 & -3.576210842691 \\ \mathrm{C} & -0.055271359338 & -1.836847443684 & -1.613986482137 \\ \mathrm{C} & -1.247032682901 & -1.929906062627 & -1.391223956088 \\ \mathrm{C} & -2.658912417155 & -2.048964785647 & -1.159046980127 \\ \mathrm{C} & -3.130607982998 & -2.709904517914 & -0.014507791737 \\ \mathrm{C} & -3.573731408723 & -1.509255107911 & -2.075148759002 \\ \mathrm{C} & -4.498907166446 & -2.807485218735 & 0.220820505056 \\ \mathrm{H} & -2.417686017941 & -3.140213146157 & 0.684707445496 \\ \mathrm{C} & -4.940732398798 & -1.615118379808 & -1.835610013982 \\ \mathrm{H} & -3.201619545895 & -0.999556424608 & -2.958635623029 \\ \mathrm{C} & -5.405717795273 & -2.257686535715 & -0.686879603816 \\ \mathrm{H} & -4.857297665734 & -3.313262303746 & 1.112146891138 \\ \mathrm{H} & -5.644631924549 & -1.188370830555 & -2.543757115682 \\ \mathrm{H} & -6.472748947135 & -2.333187588099 & 0.501287719019 \\ \mathrm{Li} & -0.116235011367 & -1.086093418387 & 0.848860471578\end{array}$




\begin{tabular}{|c|c|c|c|}
\hline $\mathrm{C}$ & 1.070629339019 & -1.951685970112 & 3.231856663867 \\
\hline $\mathrm{O}$ & 0.403625706352 & -2.429812186910 & 2.040783992664 \\
\hline $\mathrm{C}$ & 1.092008787158 & -3.589148887537 & 1.536486563307 \\
\hline $\mathrm{C}$ & 2.534762588863 & -3.382388524336 & 1.966307507804 \\
\hline $\mathrm{C}$ & 2.359999154348 & -2.774156924194 & 3.363353690355 \\
\hline $\mathrm{H}$ & 1.268647425116 & -0.884083355321 & 3.087833666576 \\
\hline $\mathrm{H}$ & 0.395157546591 & -2.080830264575 & 4.082040991331 \\
\hline $\mathrm{H}$ & 0.653915406796 & -4.486885658110 & 1.990031554184 \\
\hline $\mathrm{H}$ & 0.942594229911 & -3.620234672035 & 0.453297745281 \\
\hline $\mathrm{H}$ & 3.107401409680 & -4.312835728822 & 1.973849581291 \\
\hline $\mathrm{H}$ & 3.022761655594 & -2.669778392665 & 1.294215684065 \\
\hline $\mathrm{H}$ & 2.233950177299 & -3.563852108274 & 4.110208861836 \\
\hline $\mathrm{H}$ & 3.207645103759 & -2.152544052596 & 3.660998799259 \\
\hline $\mathrm{Si}$ & 0.145703504157 & 1.202825050019 & -0.596241100279 \\
\hline $\mathrm{C}$ & -2.501803733484 & 2.259223919406 & -0.656775676637 \\
\hline $\mathrm{C}$ & -1.803448929050 & 1.116899949567 & 1.593682766278 \\
\hline $\mathrm{O}$ & -0.590070772225 & 0.693946465013 & 0.948130979508 \\
\hline $\mathrm{O}$ & -1.376770388366 & 1.678040260885 & -1.291554649241 \\
\hline $\mathrm{C}$ & -1.506406266034 & 2.294358537501 & 2.526402305763 \\
\hline $\mathrm{H}$ & -2.411818787291 & 2.573754176530 & 3.076589704737 \\
\hline $\mathrm{H}$ & -0.740629001877 & 1.998025041994 & 3.252854458279 \\
\hline $\mathrm{H}$ & -1.146260342242 & 3.173413744529 & 1.994768790814 \\
\hline $\mathrm{C}$ & -2.259579503741 & -0.054409594133 & 2.469432587928 \\
\hline $\mathrm{H}$ & -1.474461882658 & -0.319406566948 & 3.188057780440 \\
\hline $\mathrm{H}$ & -3.160033477425 & 0.213370175511 & 3.032864548585 \\
\hline $\mathrm{H}$ & -2.503121191318 & -0.933822882897 & 1.862849844509 \\
\hline $\mathrm{C}$ & -2.235222389946 & 3.740322512821 & -0.342561531514 \\
\hline $\mathrm{H}$ & -2.153994454020 & 4.294172232348 & -1.284770567320 \\
\hline $\mathrm{H}$ & -3.056750517037 & 4.171107405071 & 0.242172949429 \\
\hline $\mathrm{H}$ & -1.300228588620 & 3.892584295851 & 0.196654729379 \\
\hline $\mathrm{C}$ & -3.656989391535 & 2.204556329772 & -1.661549646341 \\
\hline $\mathrm{H}$ & -4.556473233814 & 2.678016647265 & -1.250233613242 \\
\hline $\mathrm{H}$ & -3.372649778092 & 2.732479647814 & -2.577912320103 \\
\hline $\mathrm{H}$ & -3.893821266776 & 1.167772315724 & -1.916793548325 \\
\hline $\mathrm{C}$ & 1.040980829252 & 2.855923624144 & -0.279934992974 \\
\hline $\mathrm{C}$ & 1.387775346264 & 3.615874331454 & -1.408404816762 \\
\hline $\mathrm{C}$ & 1.344553375977 & 3.409035230955 & 0.973680143012 \\
\hline $\mathrm{C}$ & 2.003708072274 & 4.864395760677 & -1.297949914298 \\
\hline $\mathrm{H}$ & 1.163344810717 & 3.225491656450 & -2.399625947986 \\
\hline $\mathrm{C}$ & 1.956017614585 & 4.657074445773 & 1.101073406396 \\
\hline $\mathrm{H}$ & 1.090264665016 & 2.862393828079 & 1.878354533978 \\
\hline $\mathrm{C}$ & 2.288430595461 & 5.391528999061 & -0.038438781472 \\
\hline $\mathrm{H}$ & 2.257553806583 & 5.426138109671 & -2.193209815010 \\
\hline $\mathrm{H}$ & 2.172909159440 & 5.056684717844 & 2.088551885048 \\
\hline $\mathrm{H}$ & 2.764651109239 & 6.363643154377 & 0.055224161737 \\
\hline $\mathrm{H}$ & 0.554918709299 & 0.865261881200 & -2.010195926642 \\
\hline $\mathrm{C}$ & -2.899348744123 & 1.414666135745 & 0.563688980136 \\
\hline $\mathrm{H}$ & -3.749873665298 & 1.880995536760 & 1.078493869341 \\
\hline $\mathrm{H}$ & -3.251761782305 & 0.449515650822 & 0.170928967218 \\
\hline
\end{tabular}




\section{2-8. Mechanistic Studies}

\section{2-8-1. Competition Experiment}

The desired benzosilole 3a was obtained in 24\% yield accompanied with the precursor of the hydride transfer step (77\% yield), when the reaction was quenched after $5 \mathrm{~min}$ (panel 1, Scheme S2). Additionally, a competition experiment using a 1:1 mixture of 1a and 1a-D (deuterated 1a) gave a mixture of 3a and 3a-D in a ratio of 1.71:1 (panel 2, Scheme S2). Although further mechanistic studies are needed, these results together with the DFT calculations shown in Scheme S1 strongly suggest that the hydride transfer step is rate-limiting.

\section{Scheme S2.}

1) Quenching at the very early stage of the reaction
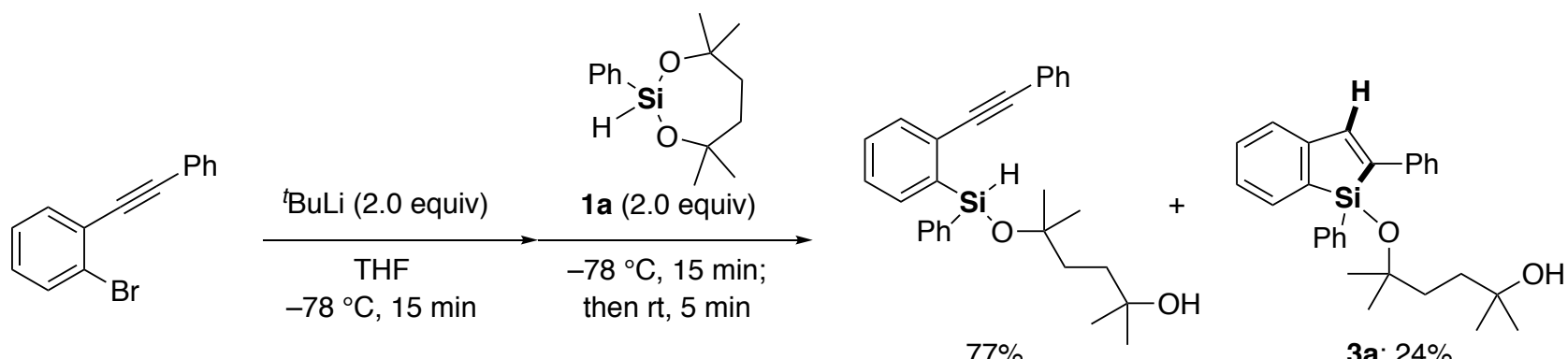

3a: $24 \%$

* Yields were determined by ${ }^{1} \mathrm{H}$ NMR spectra using mesitylene as an internal standard.

\section{2) Competition experiment using an equimolar mixture of 1a and 1a-D}
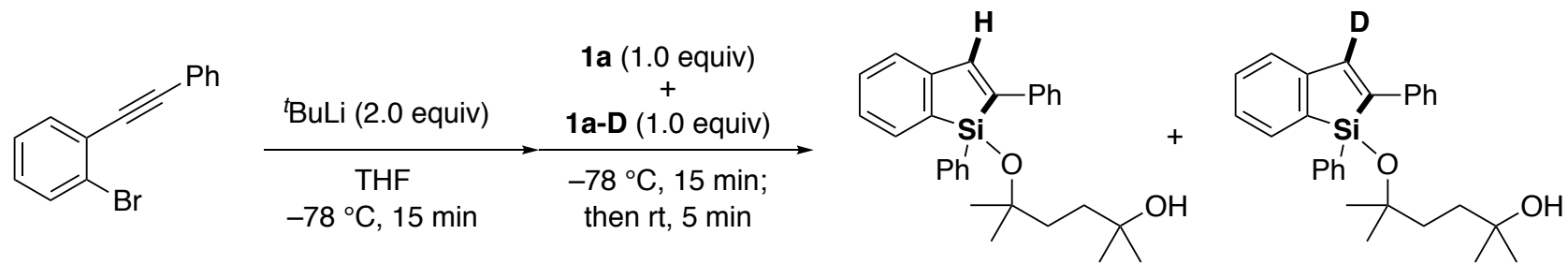

$14 \%, 3 a / 3 a-D=1.71$

* Yields was determined by ${ }^{1} \mathrm{H}$ NMR spectum using mesitylene as an internal standard. 


\section{2-8-2. Preparation of 4,4,7,7-tetramethyl-2-phenyl-1,3,2-dioxasilepane-2-d (1 a-D)}

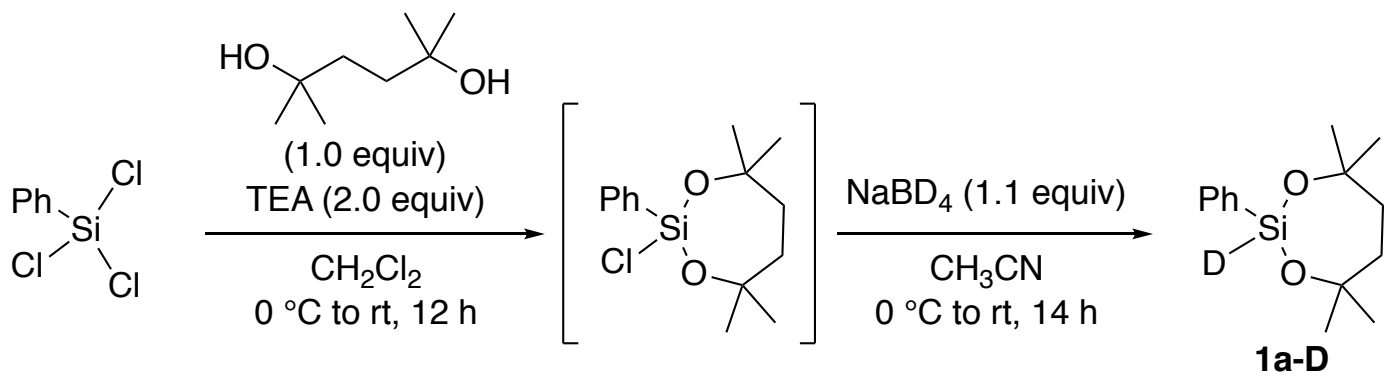

To a heat gun-dried 2-necked $200 \mathrm{~mL}$ egg-plant-shaped flask were added trichloro(phenyl)silane (2.12 g, $10.0 \mathrm{mmol}$, 1.0 equiv) and $\mathrm{CH}_{2} \mathrm{Cl}_{2}(10 \mathrm{~mL})$. After the solution was cooled to $0{ }^{\circ} \mathrm{C}$, triethylamine $(2.8 \mathrm{~mL}, 20.0 \mathrm{mmol}, 2.0$ equiv) was added. To the mixture was added a solution of 2,5-dimethylhexane-2,5-diol (1.46 g, $10.0 \mathrm{mmol}, 1.0$ equiv) in $\mathrm{CH}_{2} \mathrm{Cl}_{2}(80 \mathrm{~mL})$ dropwise at $0{ }^{\circ} \mathrm{C}$. The resulting mixture was allowed to warm to room temperature and vigorously stirred for $12 \mathrm{~h}$. After volatiles were removed by evaporation, the residue was suspended in hexane and stirred vigorously for 12 hours. Filtration through a pad of Celite to remove ammonium chloride gave a clear colorless solution, which was concentrated in vacuo to give 2-chloro-4,4,7,7-tetramethyl-2-phenyl-1,3,2-dioxasilepane as colorless oil. This compound was used for the next step without further purification.

${ }^{1} \mathrm{H}$ NMR (400 MHz, $\left.\mathbf{C D C l}_{3}\right): \delta 1.27(\mathrm{~s}, 6 \mathrm{H}), 1.43(\mathrm{~s}, 6 \mathrm{H}), 1.79-1.99(\mathrm{~m}, 4 \mathrm{H}), 7.31-7.40(\mathrm{~m}, 3 \mathrm{H}), 7.68-7.71(\mathrm{~m}, 2 \mathrm{H})$. ${ }^{13}$ C NMR (100 MHz, CDCl ): $\delta 29.6$ (br), 30.7 (br), 37.3, 77.4, 127.8, 130.7, 133.1, 133.9. IR (ATR-IR): 2977, 2937, 1594, 1431, 1386, 1369, 1277, 1244, 1215, 1169, 1124, 1040, 1027, 998, 853, 816, 741, 712, 696, 647, 555, 519, 474, $428,404 \mathrm{~cm}^{-1}$. MS (GC-MS, EI): $m / z$ calculated for $\mathrm{C}_{15} \mathrm{H}_{21} \mathrm{ClO}_{3} \mathrm{Si}^{+}[\mathrm{M}]^{+}=284.1$, found: 284.1 .

To a heat gun-dried 2-necked $30 \mathrm{~mL}$ egg-plant-shaped flask were added $\mathrm{NaBD}_{4}(138 \mathrm{mg}, 3.3 \mathrm{mmol}, 1.1 \mathrm{equiv})$ and $\mathrm{CH}_{3} \mathrm{CN}(5 \mathrm{~mL})$. After the suspension was cooled to $0{ }^{\circ} \mathrm{C}$, 2-chloro-4,4,7,7-tetramethyl-2-phenyl-1,3,2-dioxasilepane ( $855 \mathrm{mg}, 3.0 \mathrm{mmol}, 1.0$ equiv) was added dropwise. The resulting mixture was allowed to warm to room temperature and stirred for $14 \mathrm{~h}$. The reaction was quenched with sat. $\mathrm{NH}_{4} \mathrm{Cl}$ aq., followed by extraction with hexane $\mathrm{x} 3$. The combined organic layer was washed with water $\mathrm{x} 3$, dried over $\mathrm{Na}_{2} \mathrm{SO}_{4}$, filtered and concentrated in vacuo. The residue was purified by distillation with a Kugelrohr apparatus at $160^{\circ} \mathrm{C}$ under a pressure of $1.0 \mathrm{mmHg}$ to give $\mathbf{1 a - D}(380 \mathrm{mg}$, $1.51 \mathrm{mmol})$ in 50\% yield as colorless oil. ${ }^{1} \mathbf{H}$ NMR (400 $\left.\mathbf{~ M H z}, \mathbf{C D C l}_{3}\right): \delta 1.30(\mathrm{~s}, 6 \mathrm{H}), 1.39(\mathrm{~s}, 6 \mathrm{H}), 1.87$ (brs, 4H), 7.34-7.42 (m, 3H), 7.64-7.67 (m, 2H). ${ }^{13} \mathbf{C}$ NMR (100 MHz, $\left.\mathbf{C D C l}_{3}\right): \delta 29.8$ (br), 30.8 (br), 37.9, 75.4, 127.8, 130.2, 133.9, $135.1(\mathrm{t}, J=2.9 \mathrm{~Hz})$. IR (ATR-IR): 2973, 2932, 1564, 1430, 1385, 1367, 1276, 1242, 1212, 1185, 1123, 1025, $853,804,742,716,700,628,596,537,495,464,454,408 \mathrm{~cm}^{-1}$. HRMS (ESI): $\mathrm{m} / z$ calculated for $\mathrm{C}_{15} \mathrm{H}_{25} \mathrm{DNaO}_{3} \mathrm{Si}^{+}$ $[\mathrm{M}+\mathrm{MeOH},+\mathrm{Na}]^{+}=306.1606$, found: 306.1601 . 


\section{NMR Spectra}

4,4,7,7-tetramethyl-2-phenyl-1,3,2-dioxasilepane (1a)
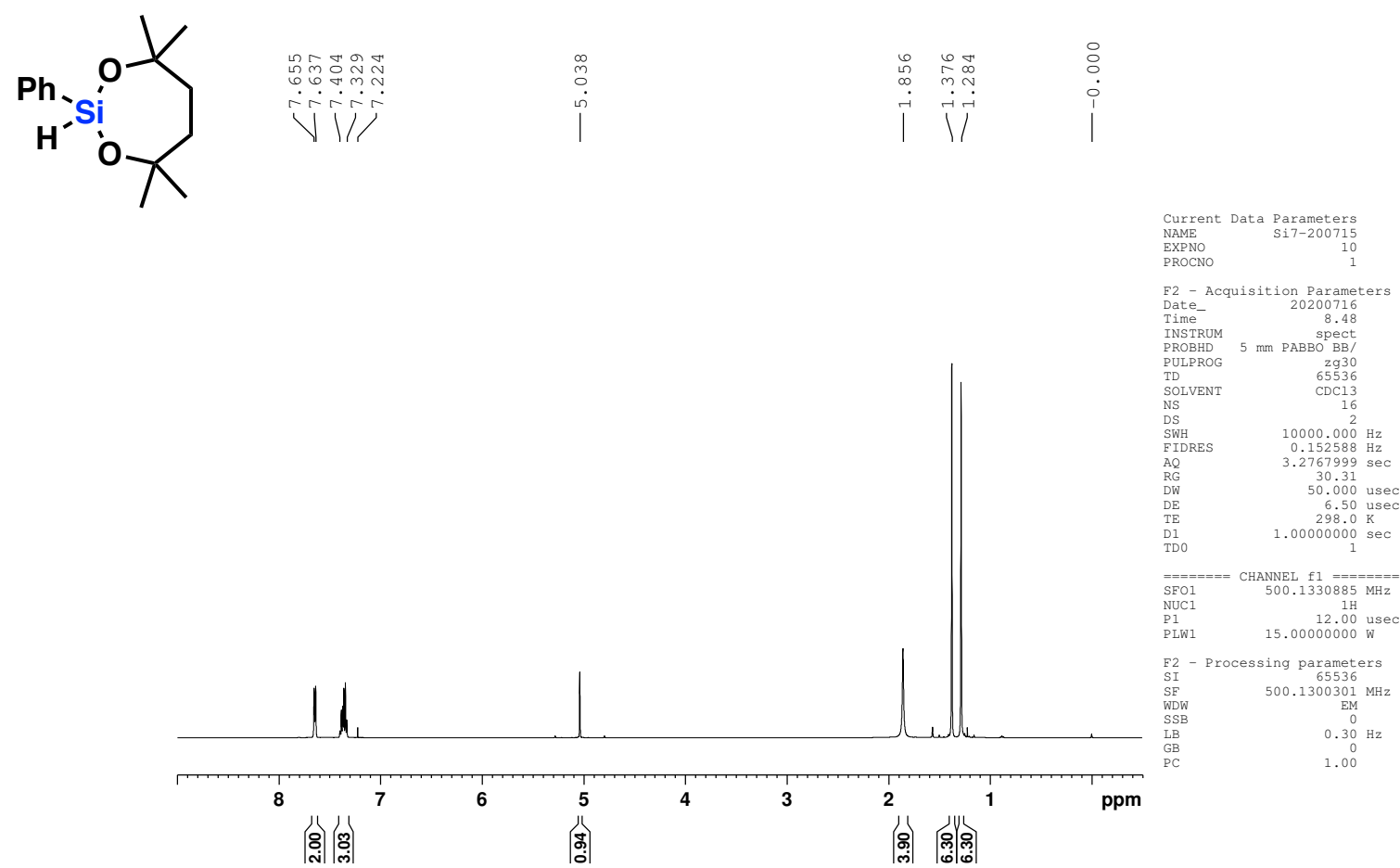

:

敌

|
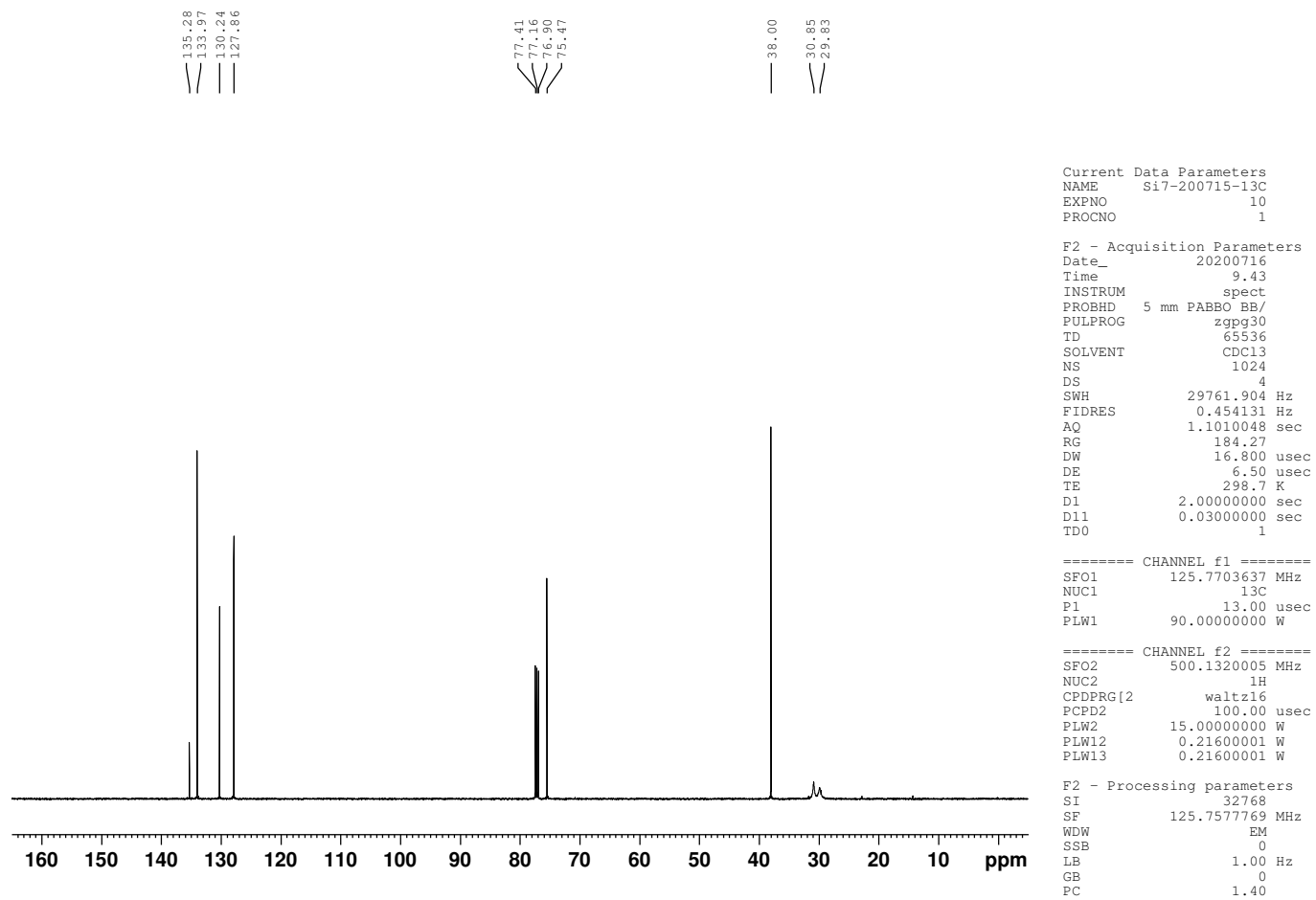


\section{4,4,6,6-tetramethyl-2-phenyl-1,3,2-dioxasilinane (1b)}
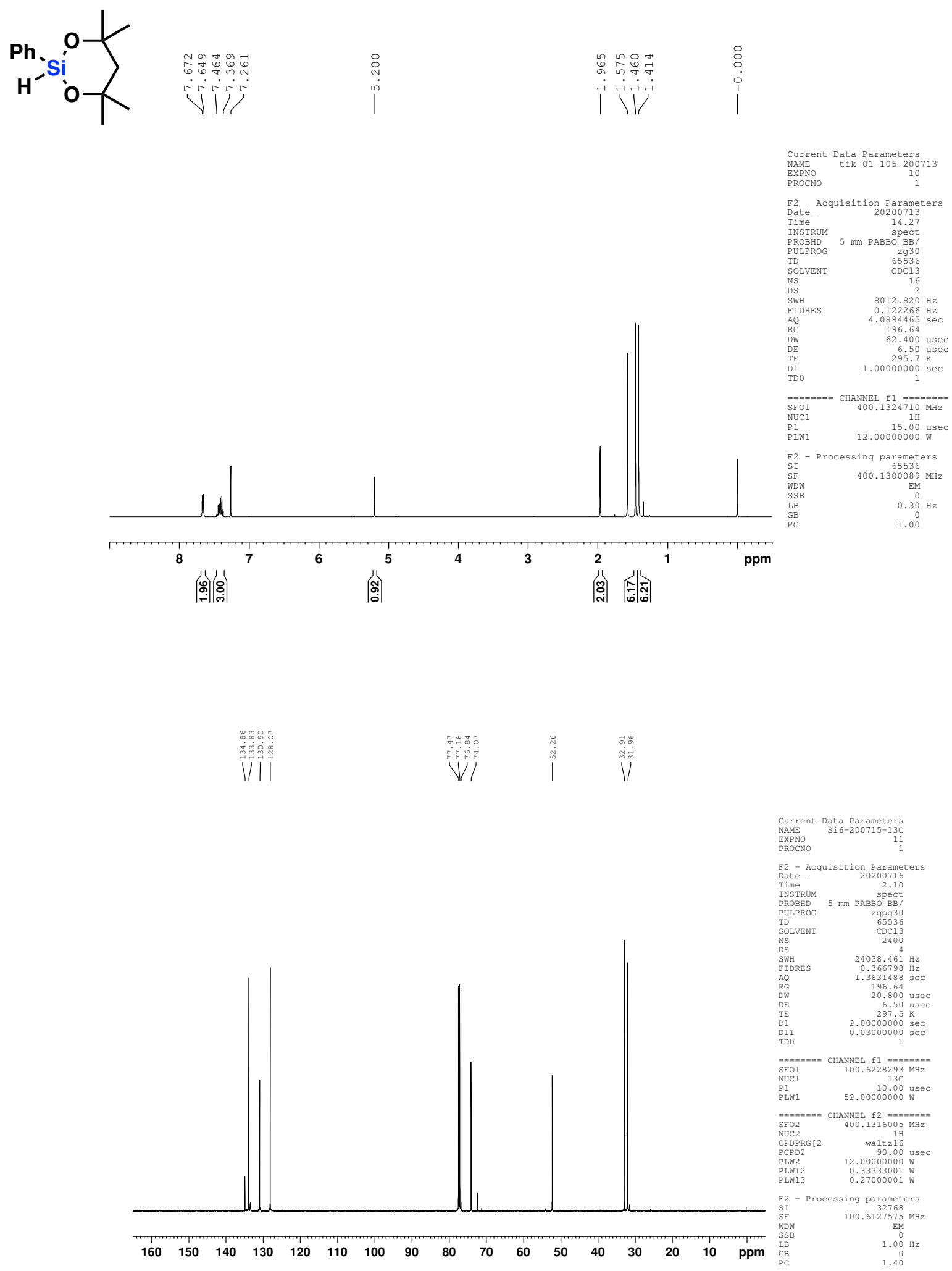

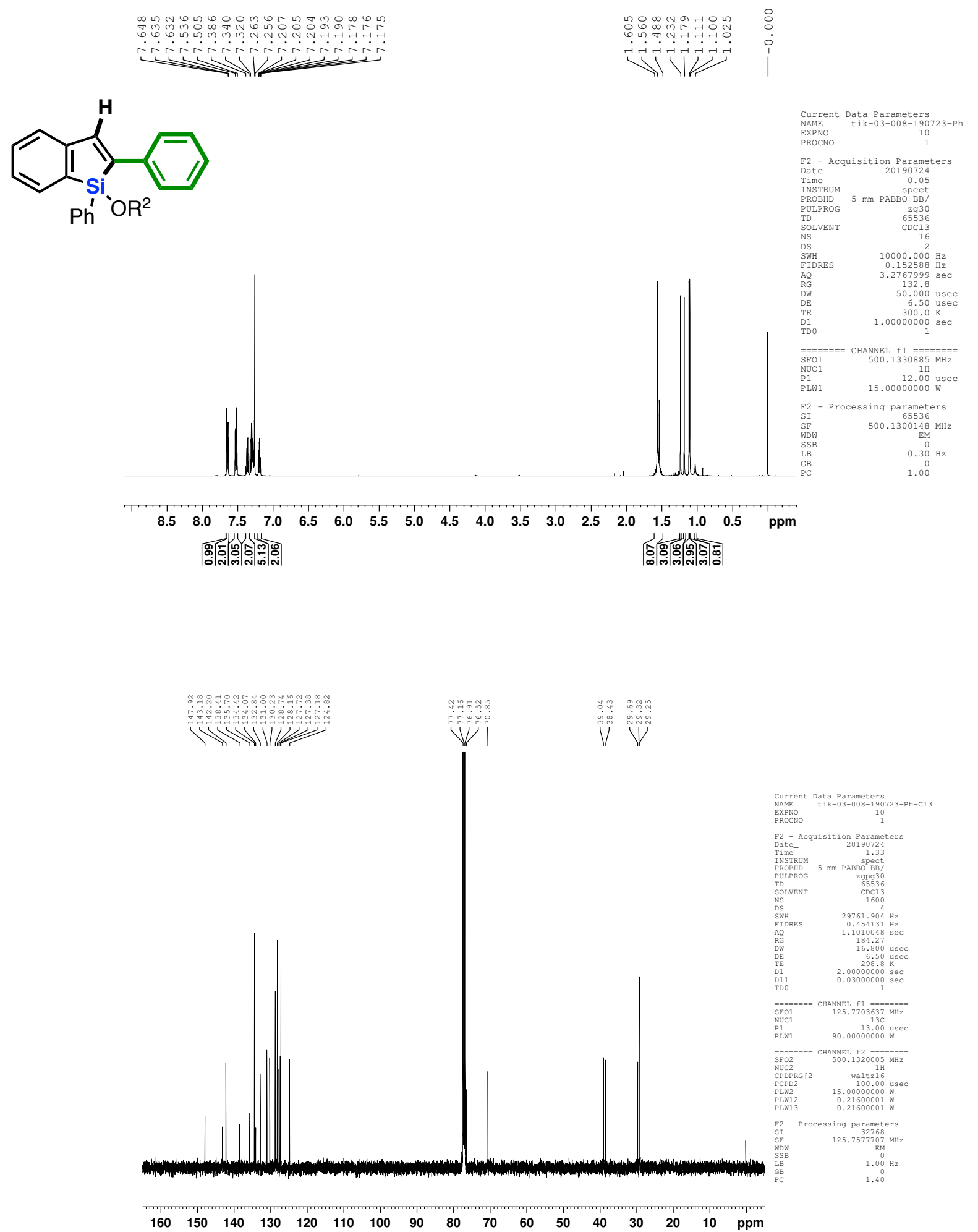


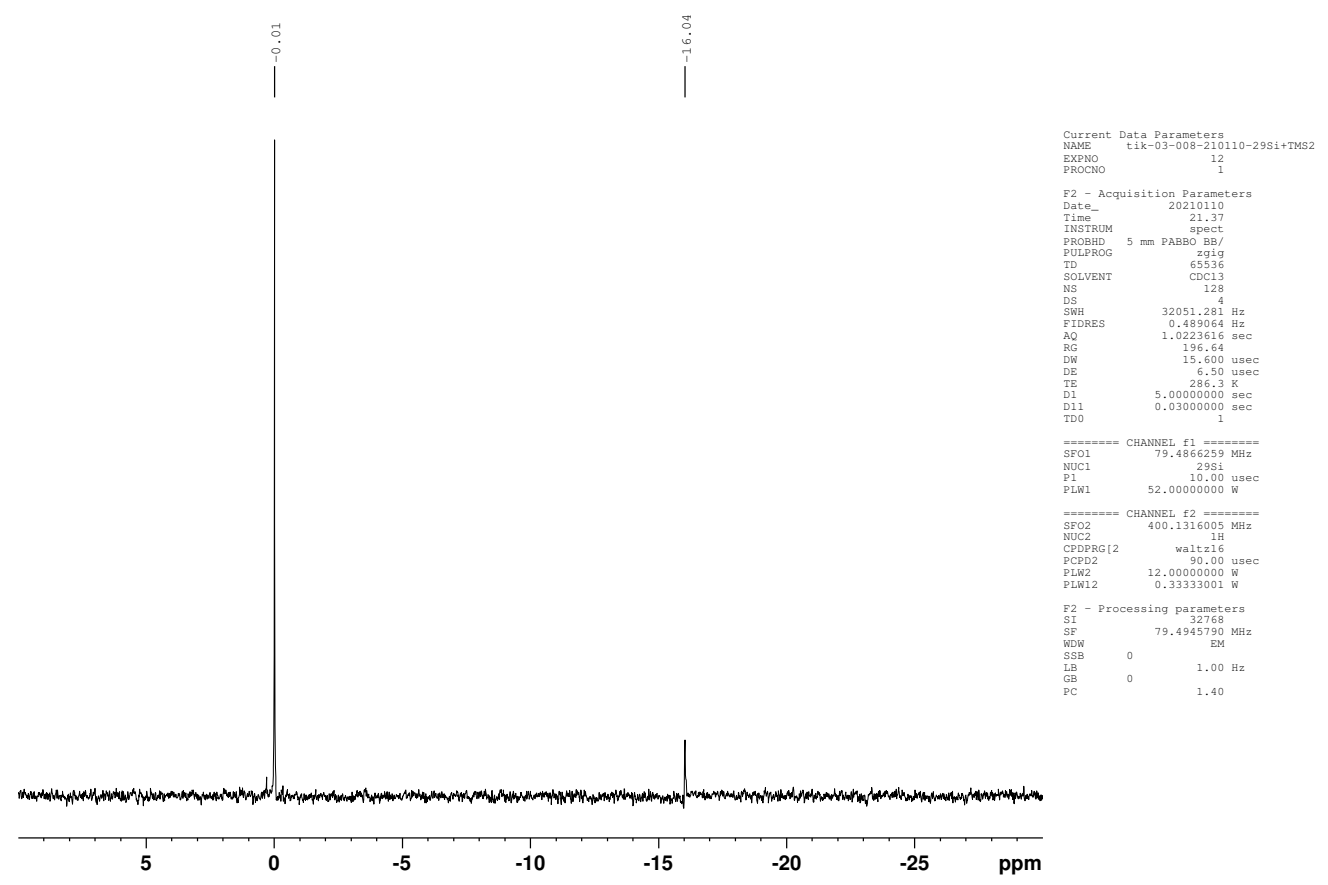




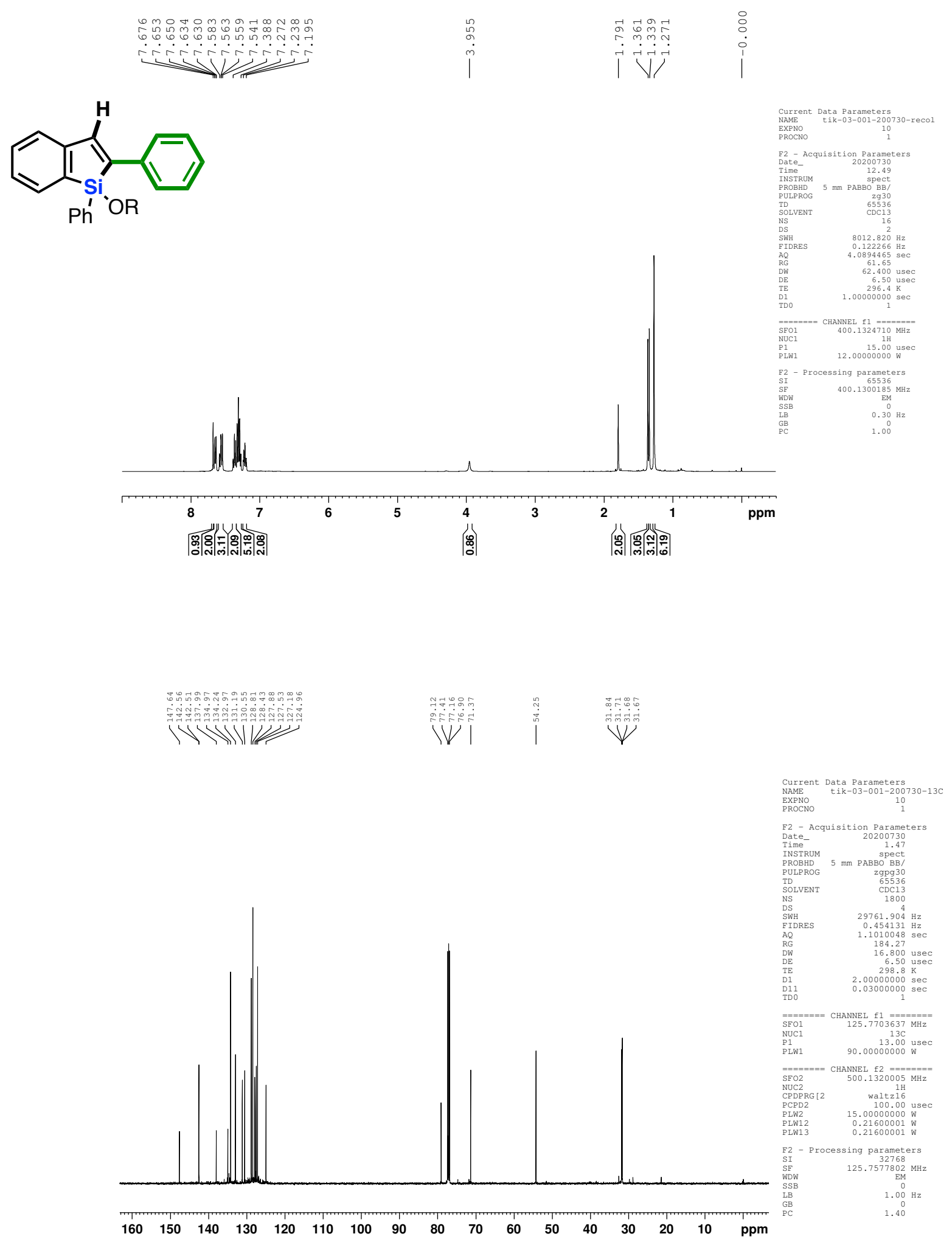



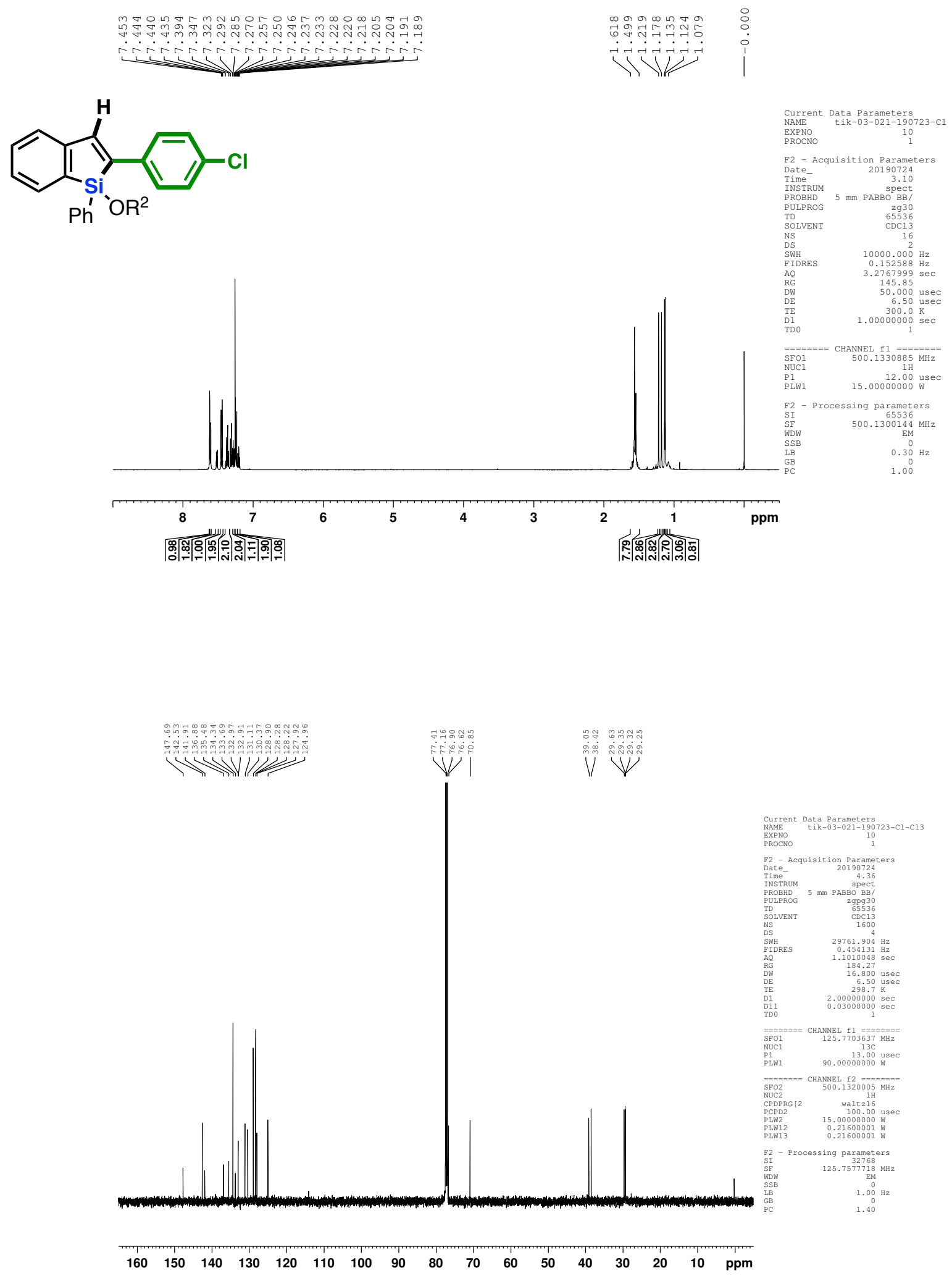


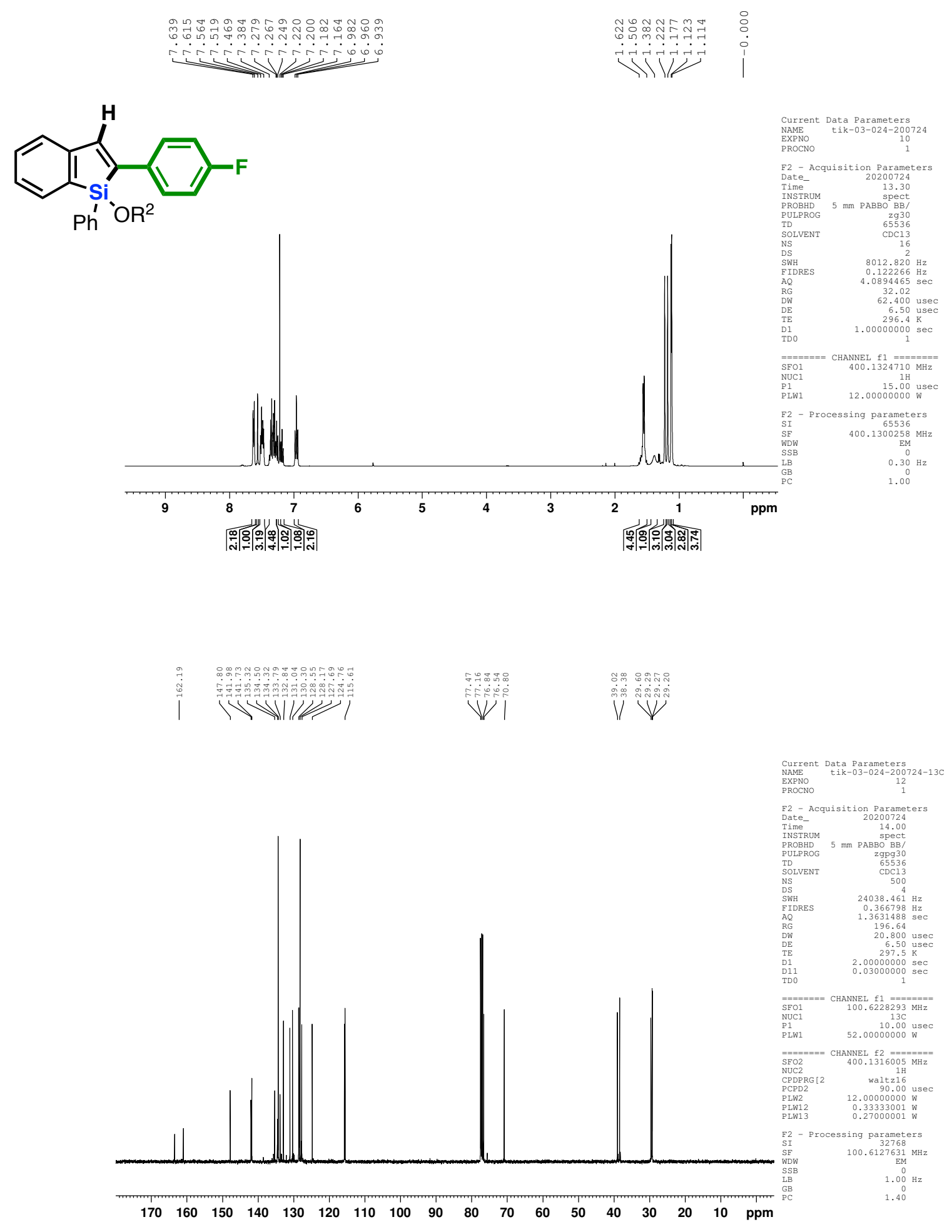




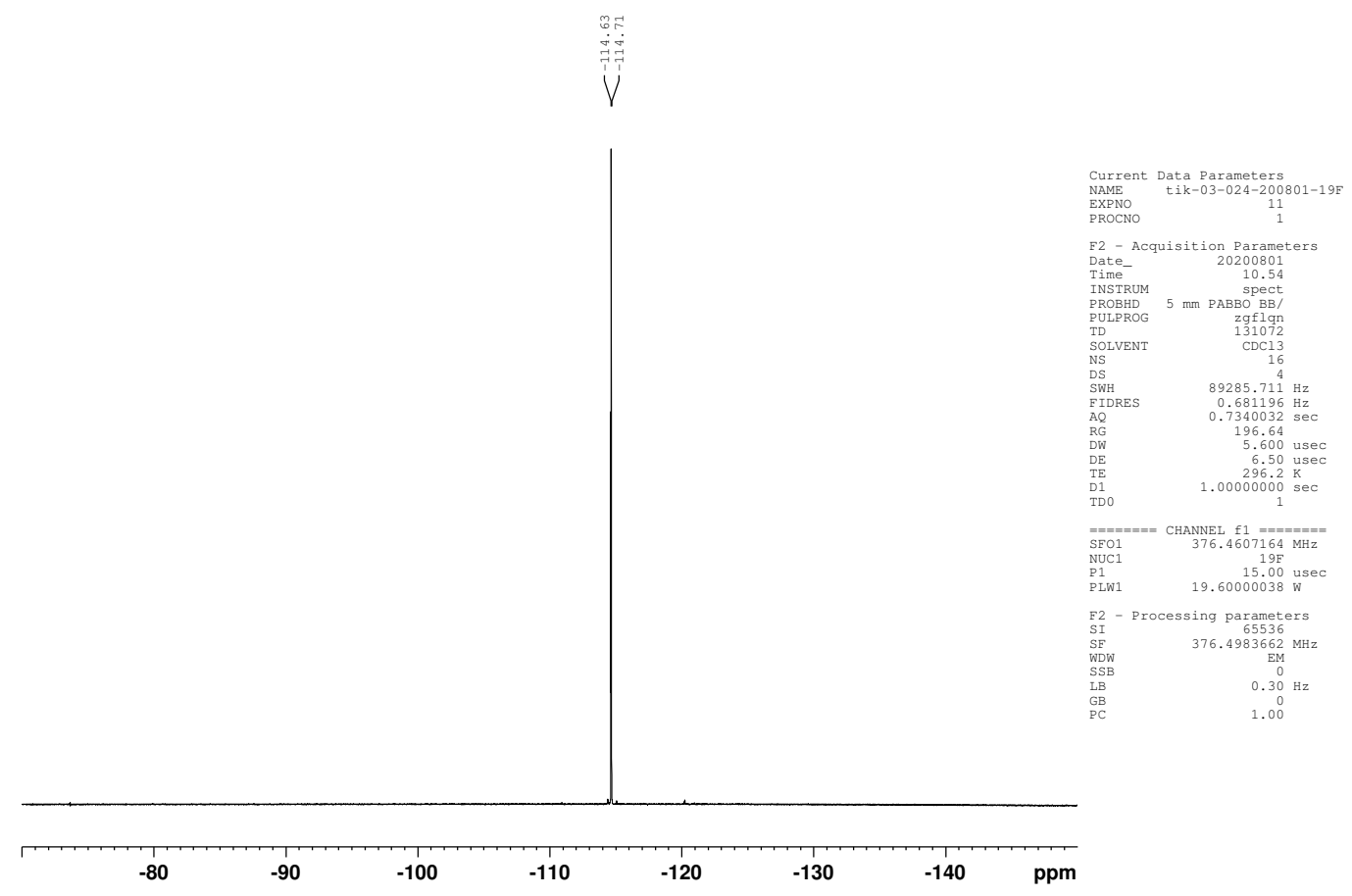



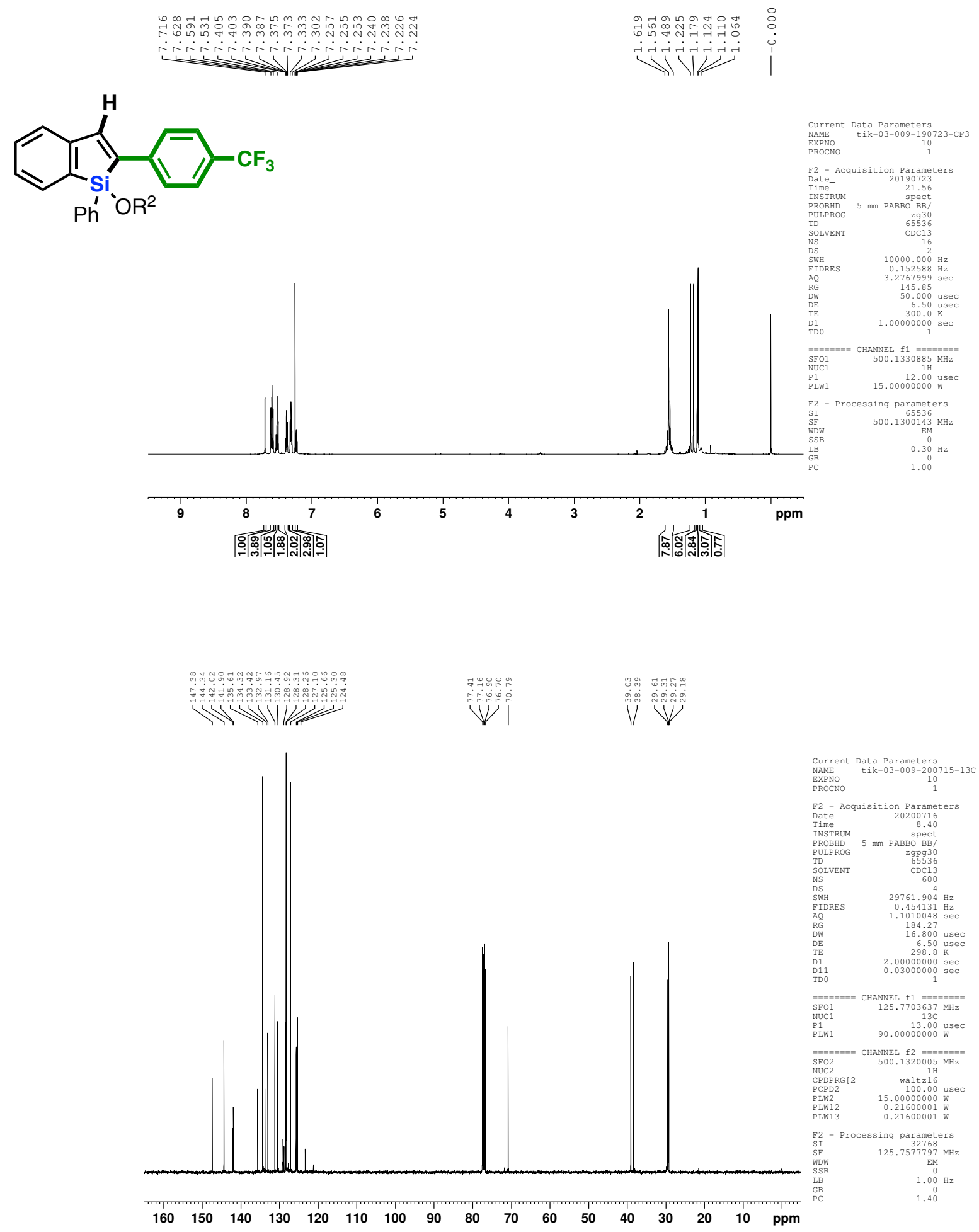


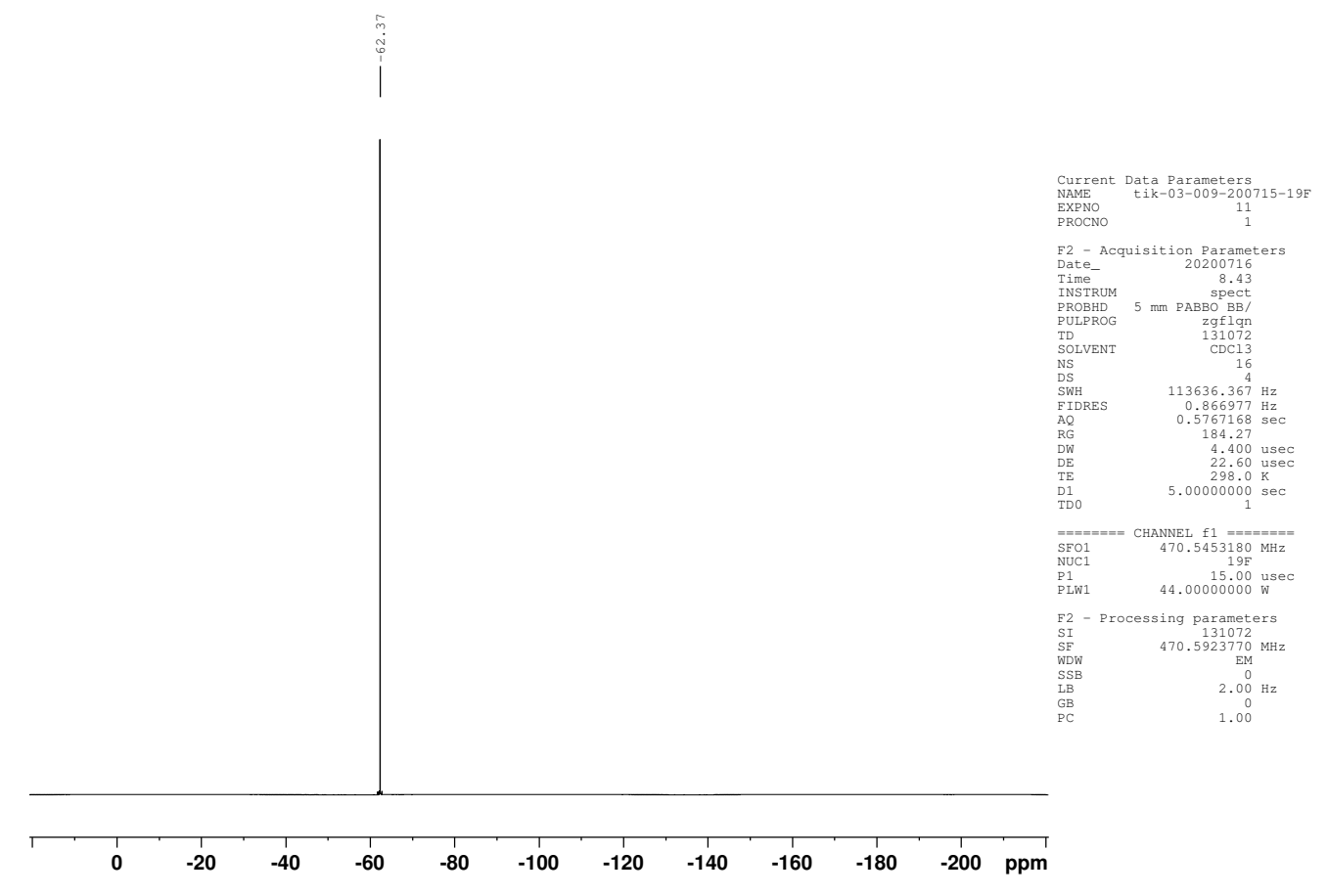




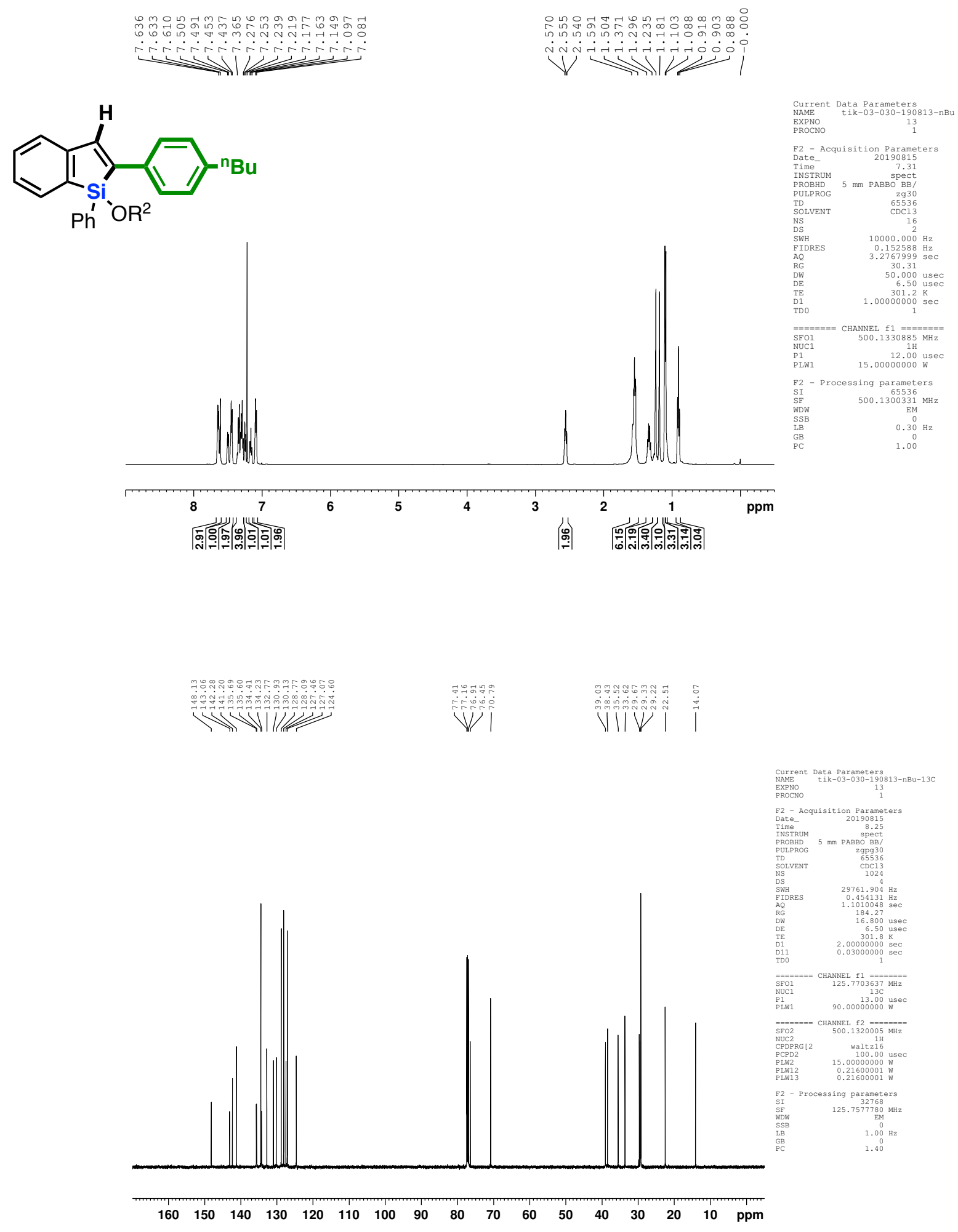




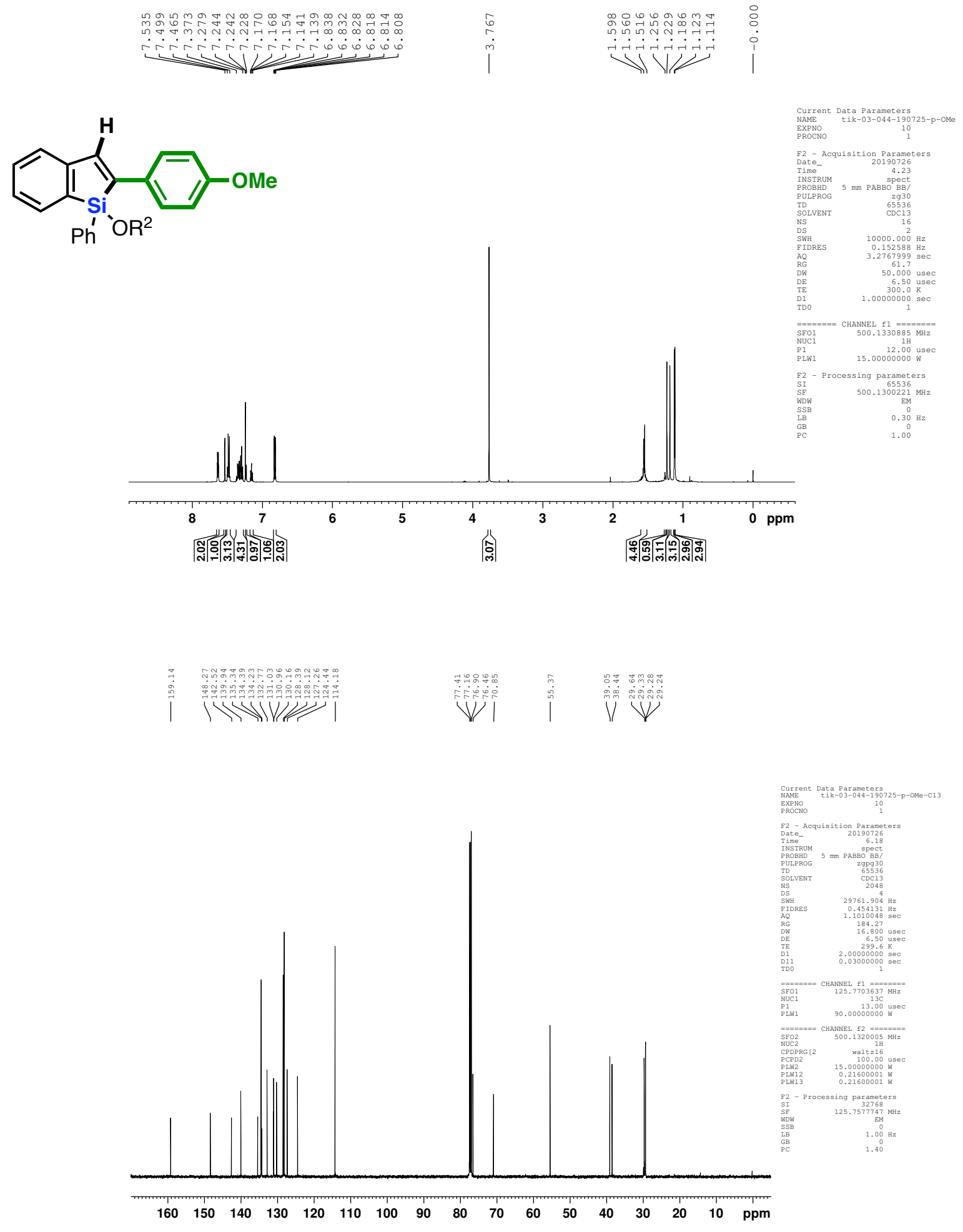




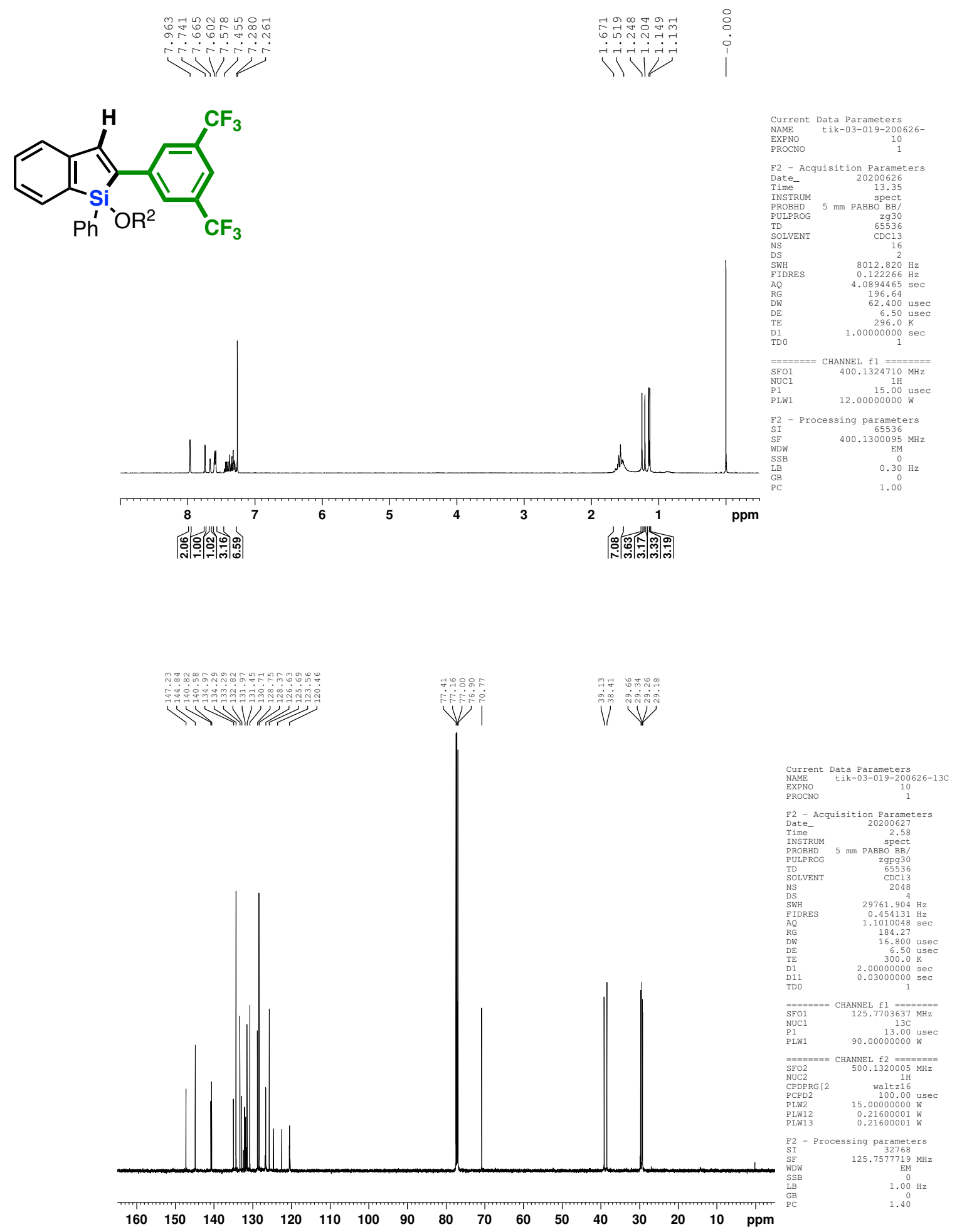




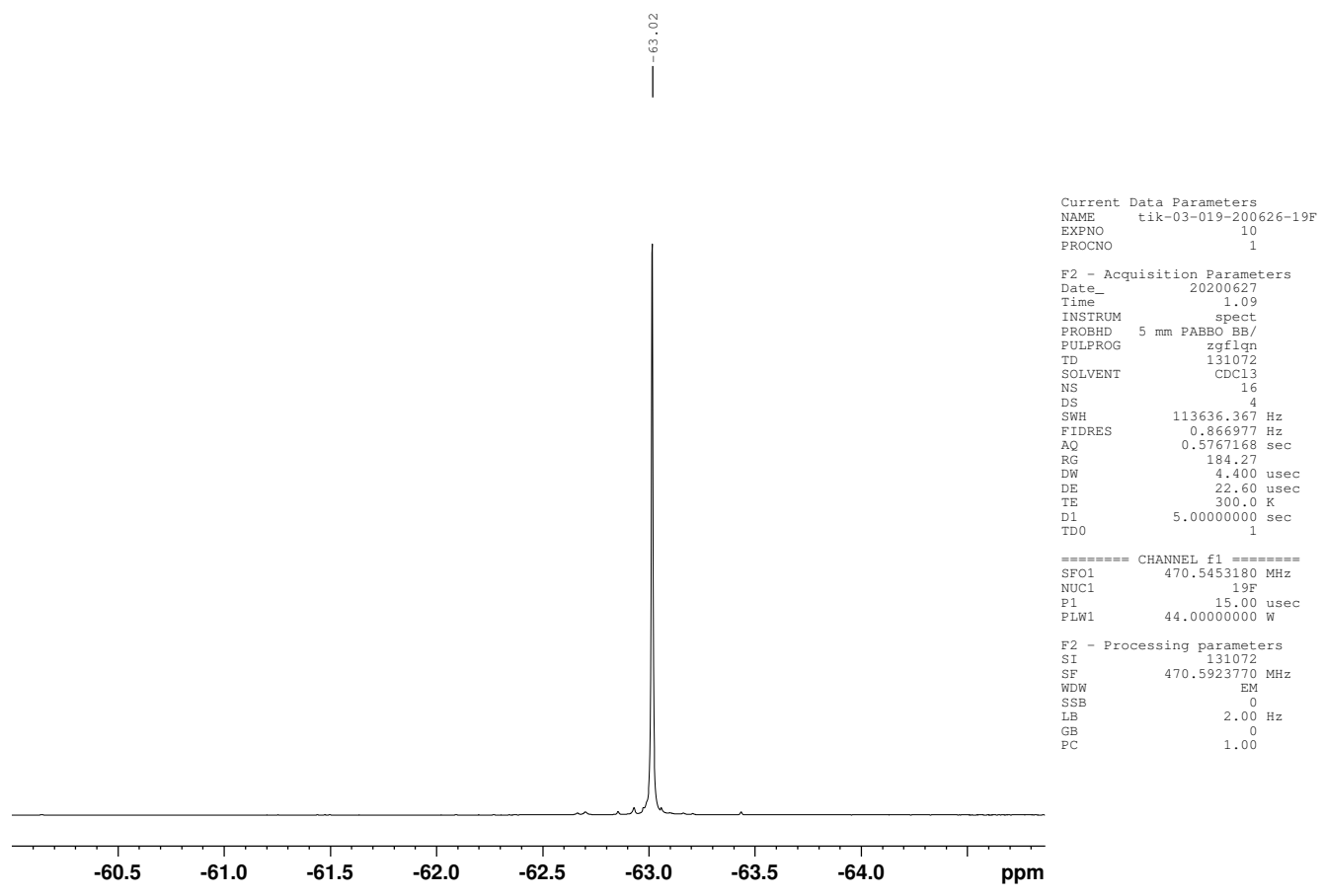




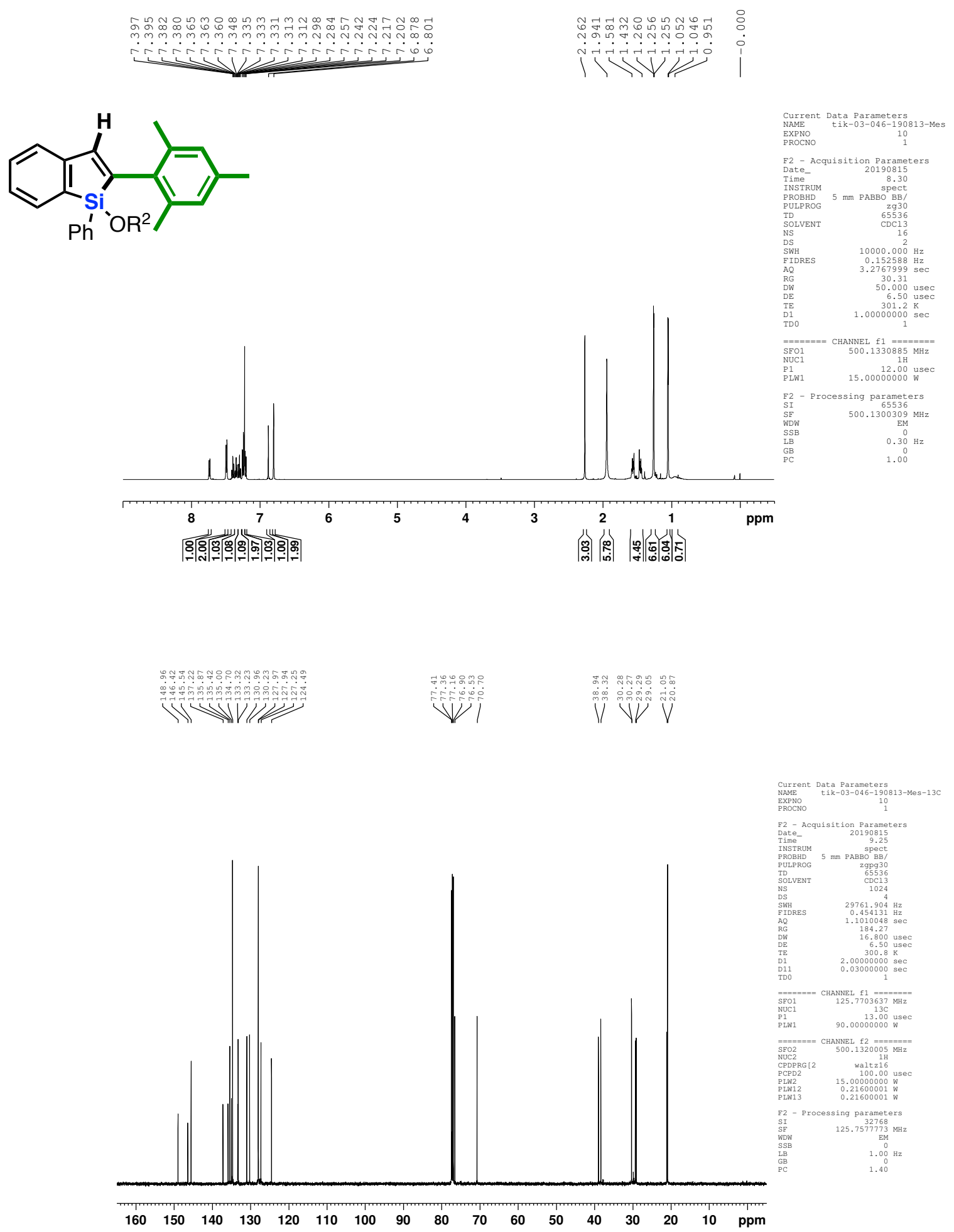


<smiles>[R7]O[Si]1(c2cccc3ccccc23)C(c2cccc3ccccc23)=C2c3ccccc3[Si]21c1ccccc1</smiles>

$\mathrm{Ph}{ }^{\prime} \mathrm{OR}{ }^{2}$ VII
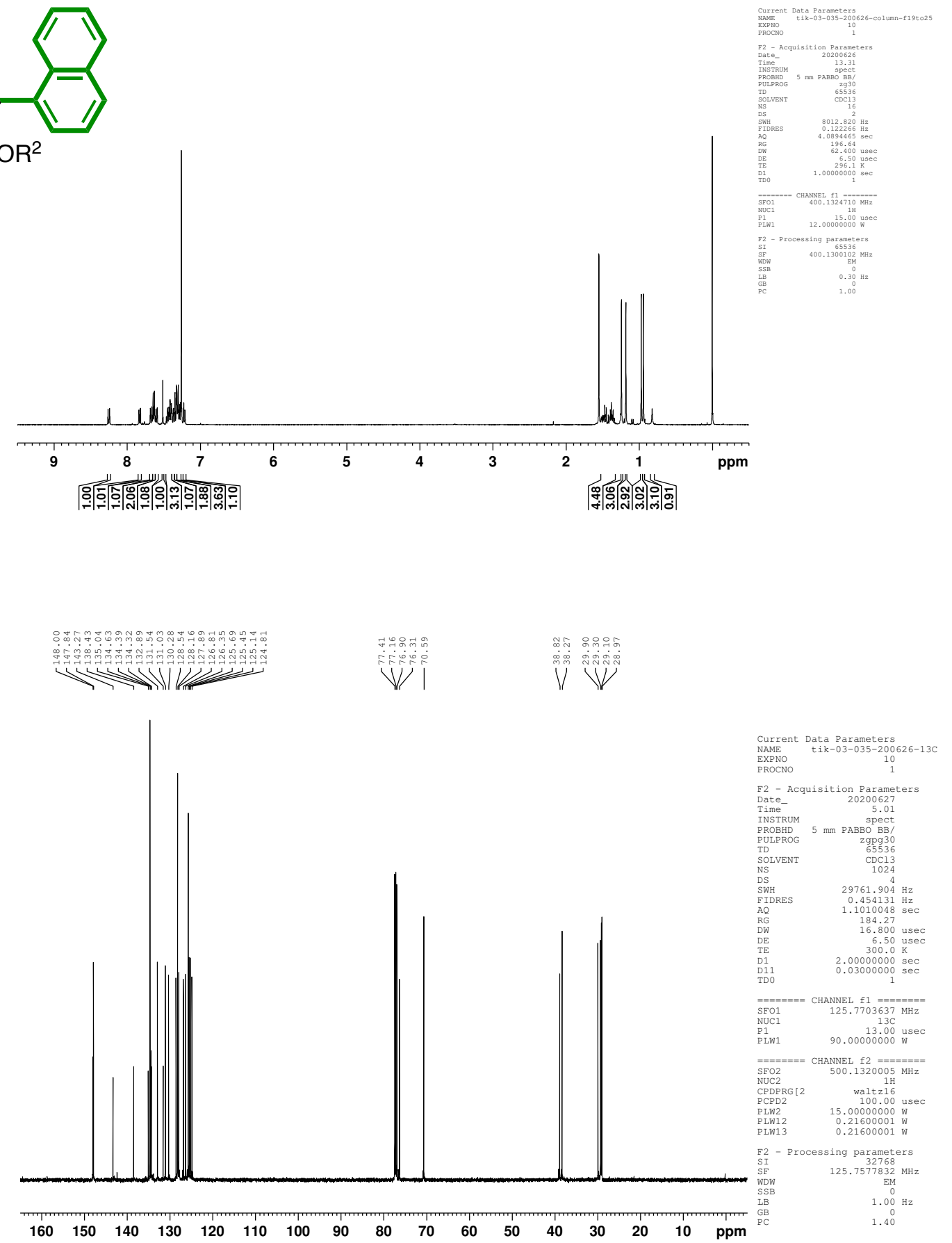

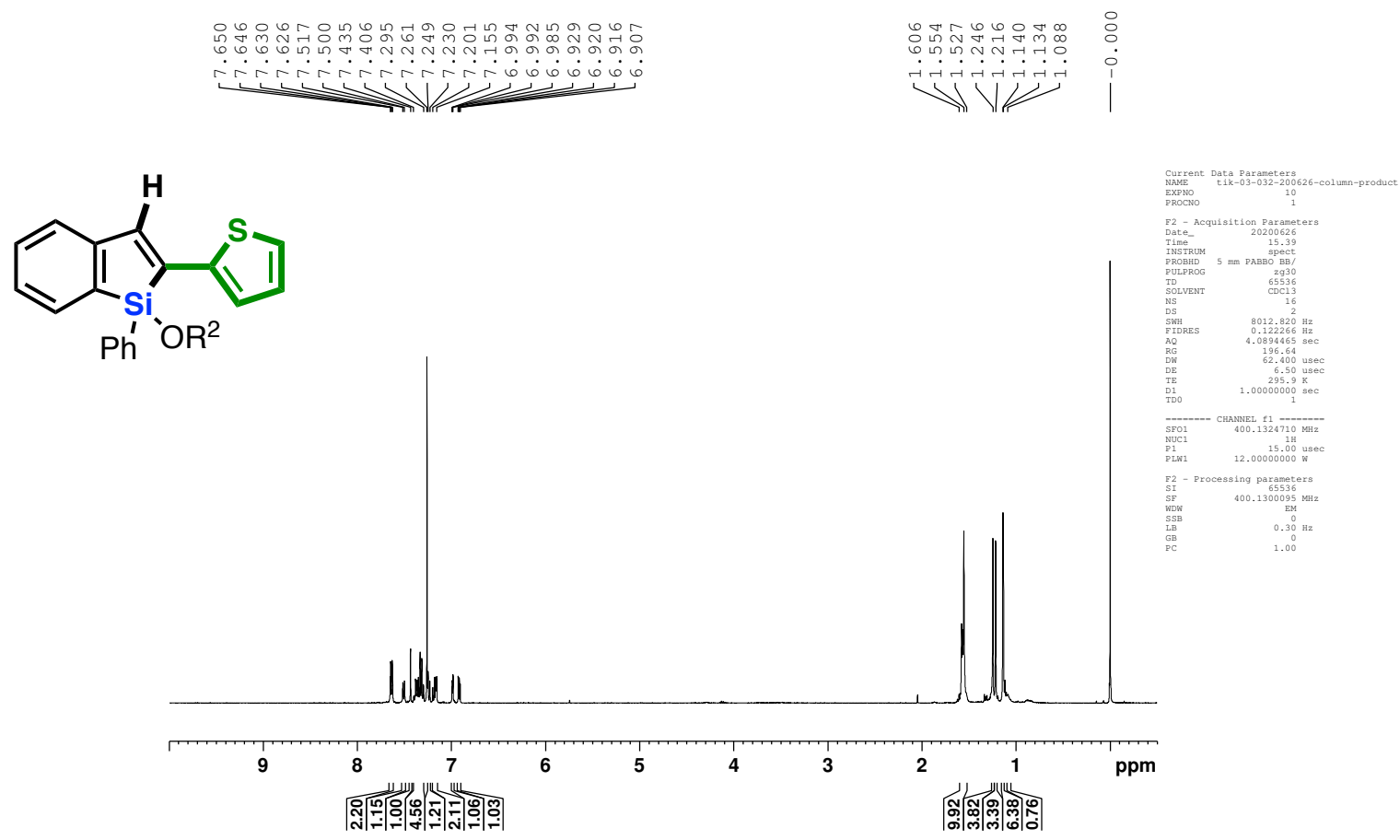

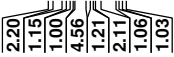

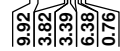
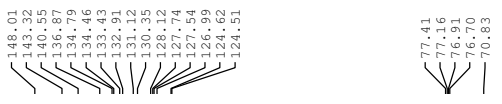

$\prod^{2}$
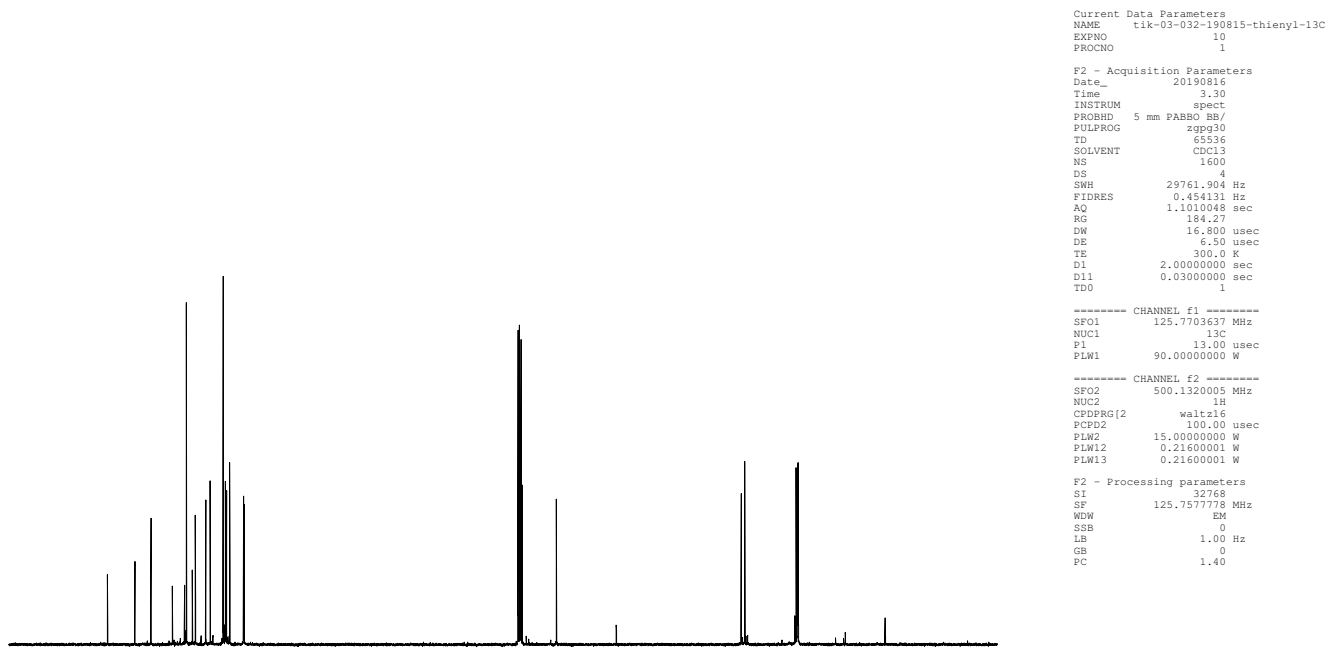

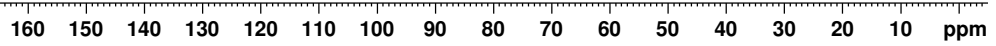




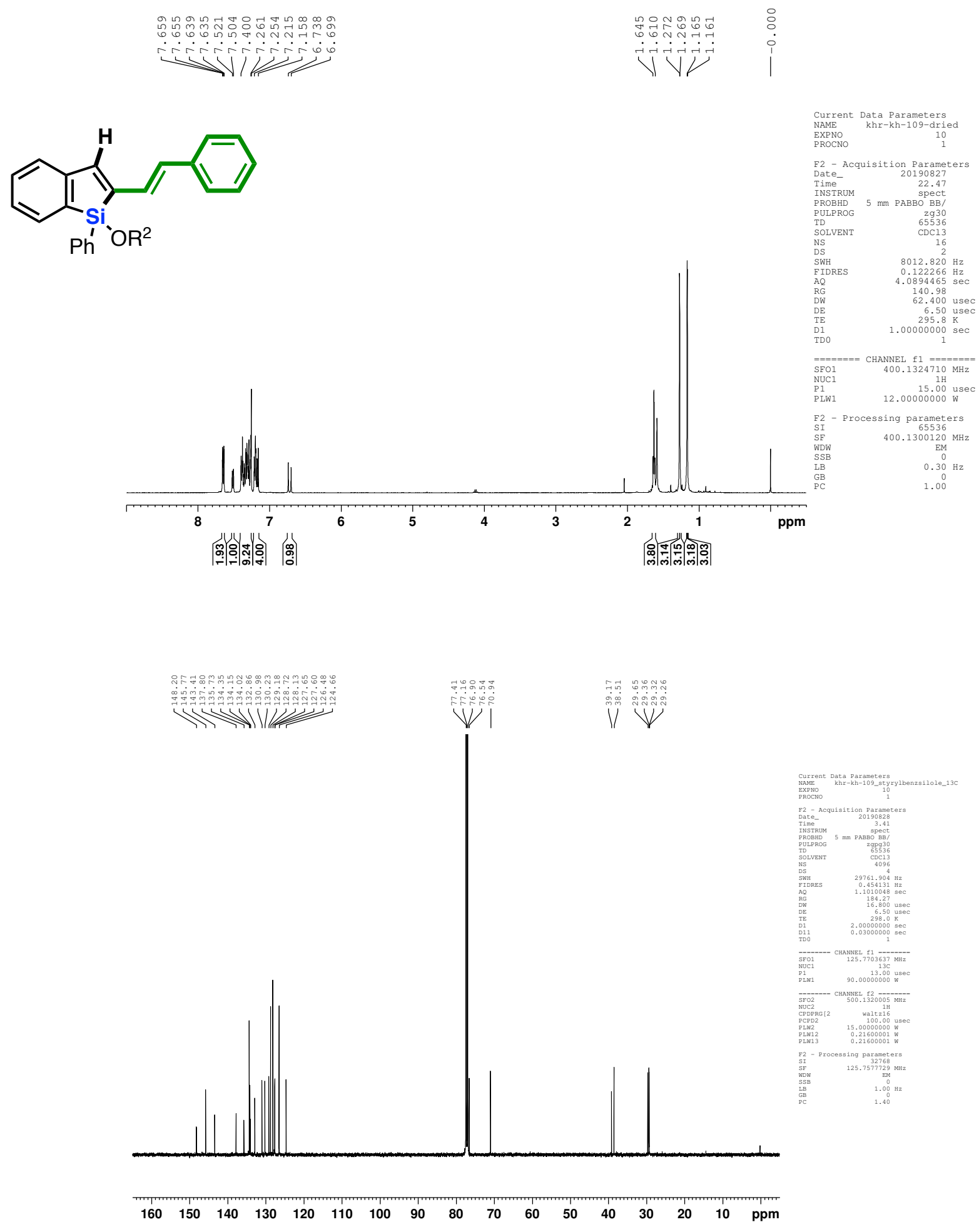




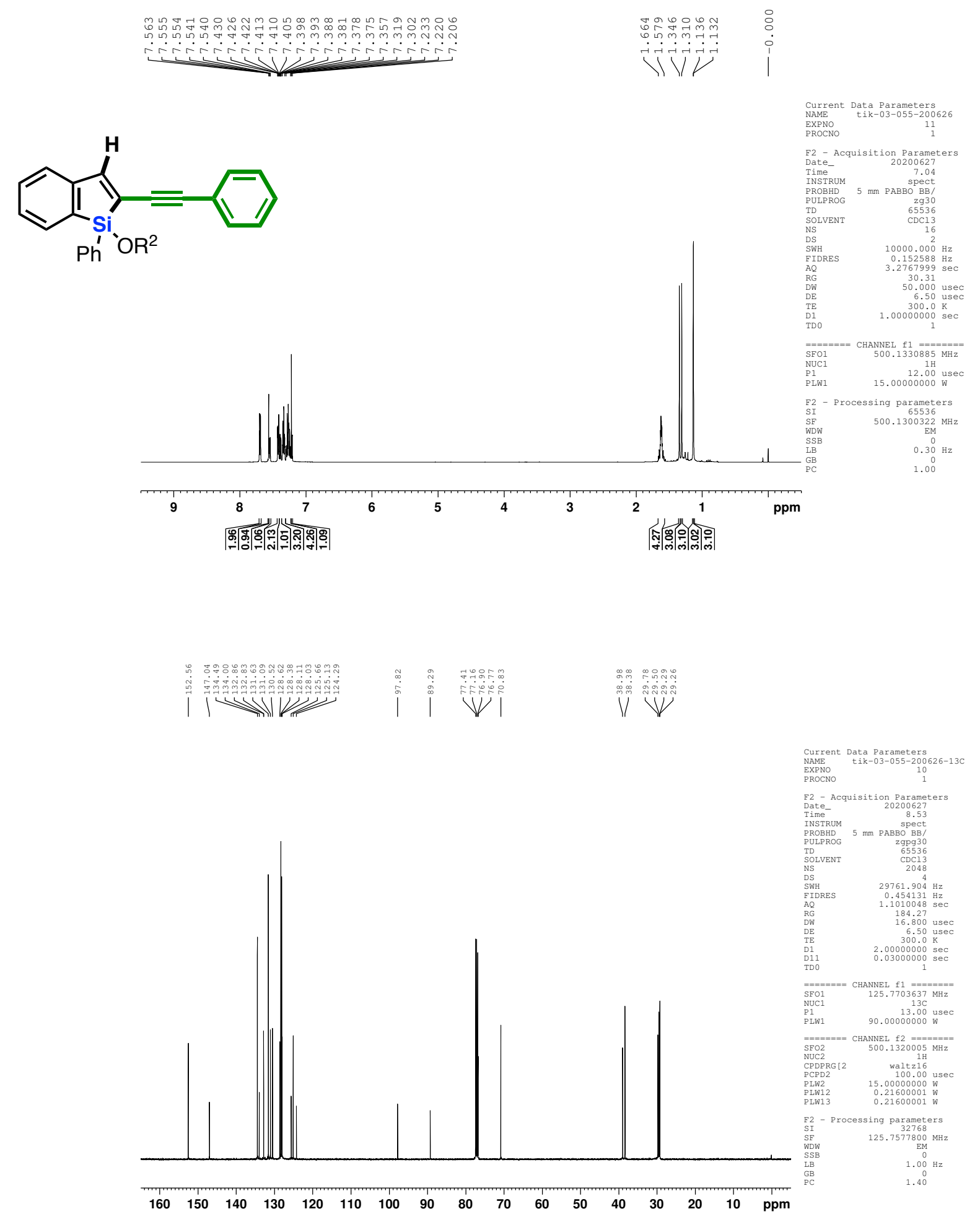




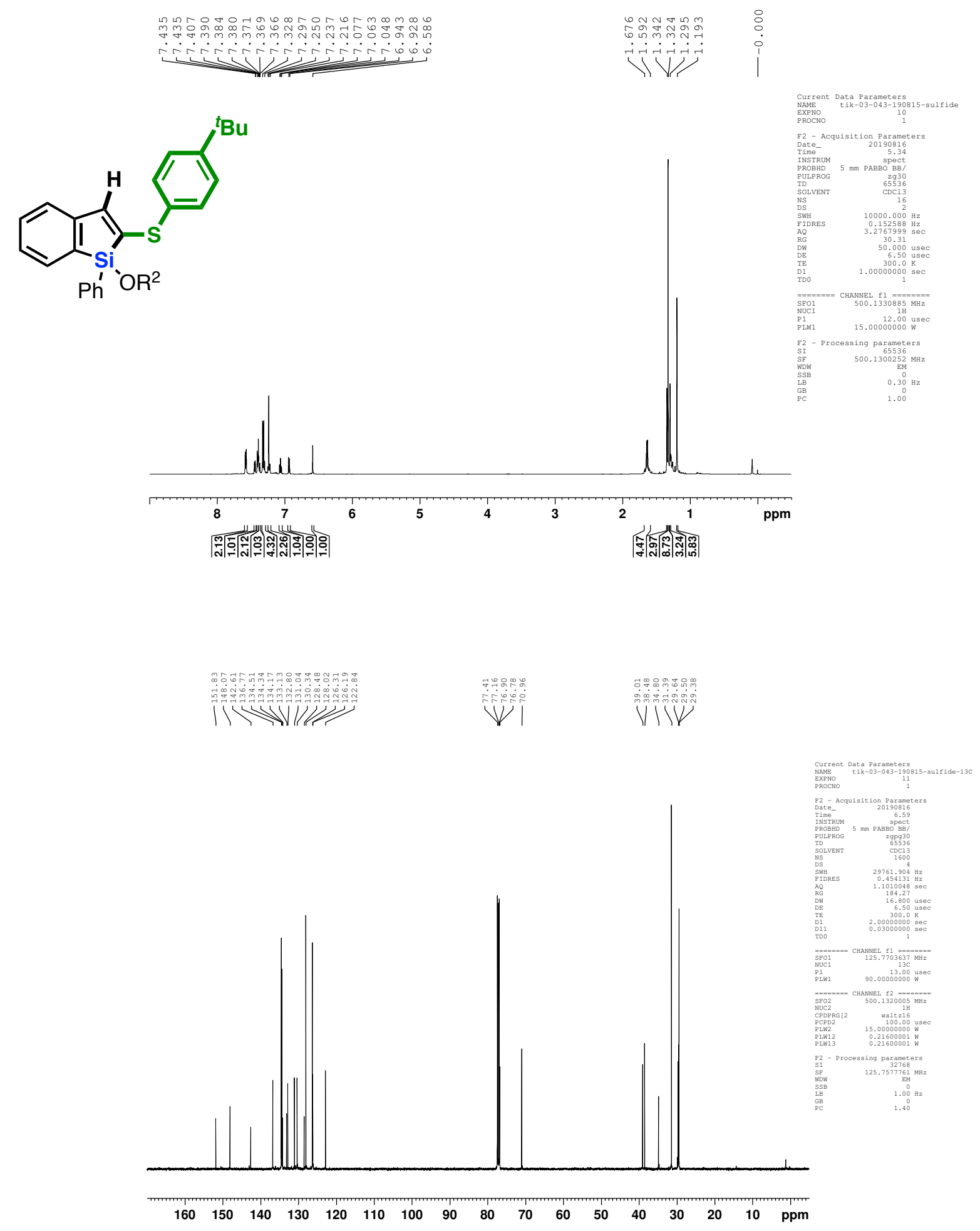




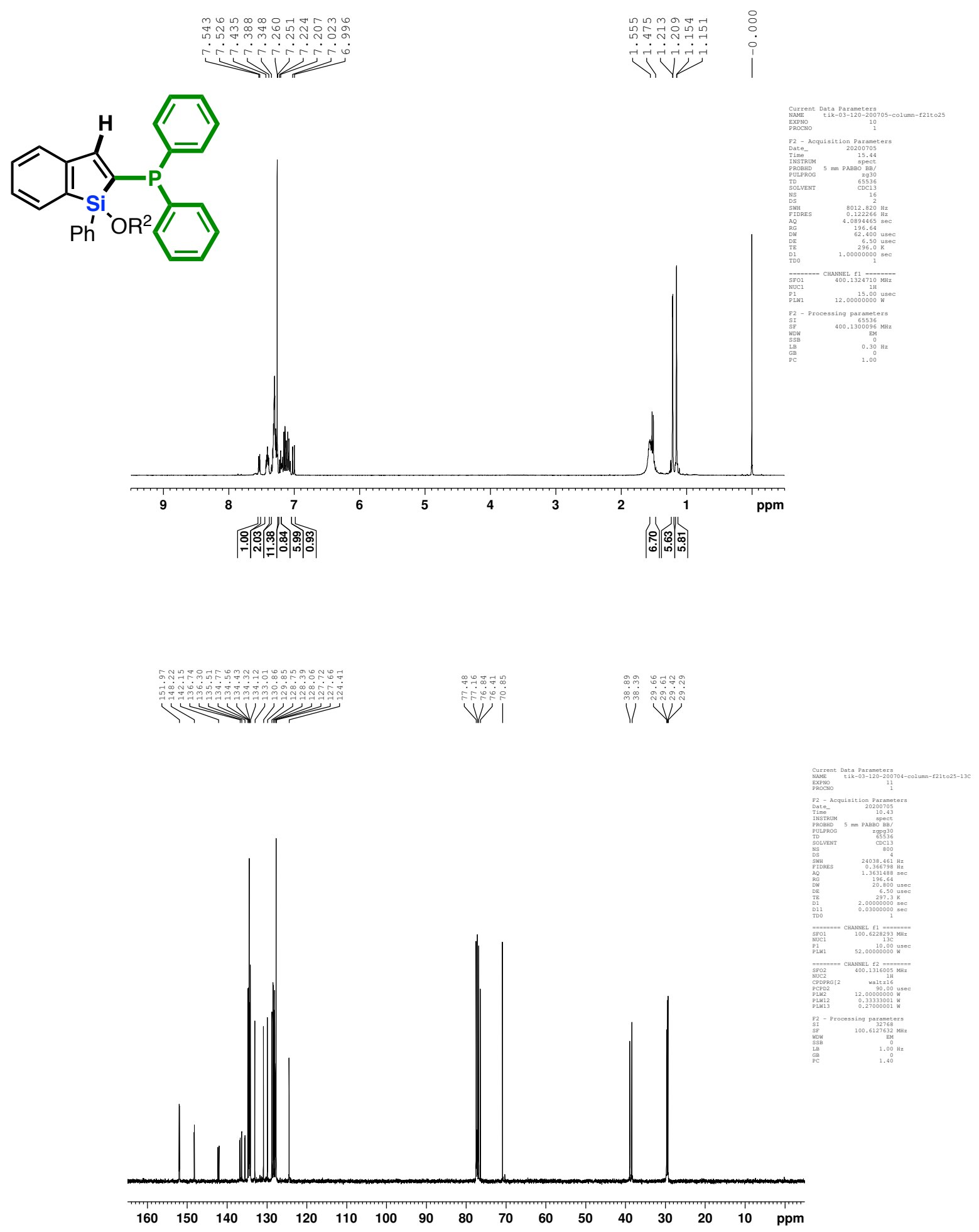




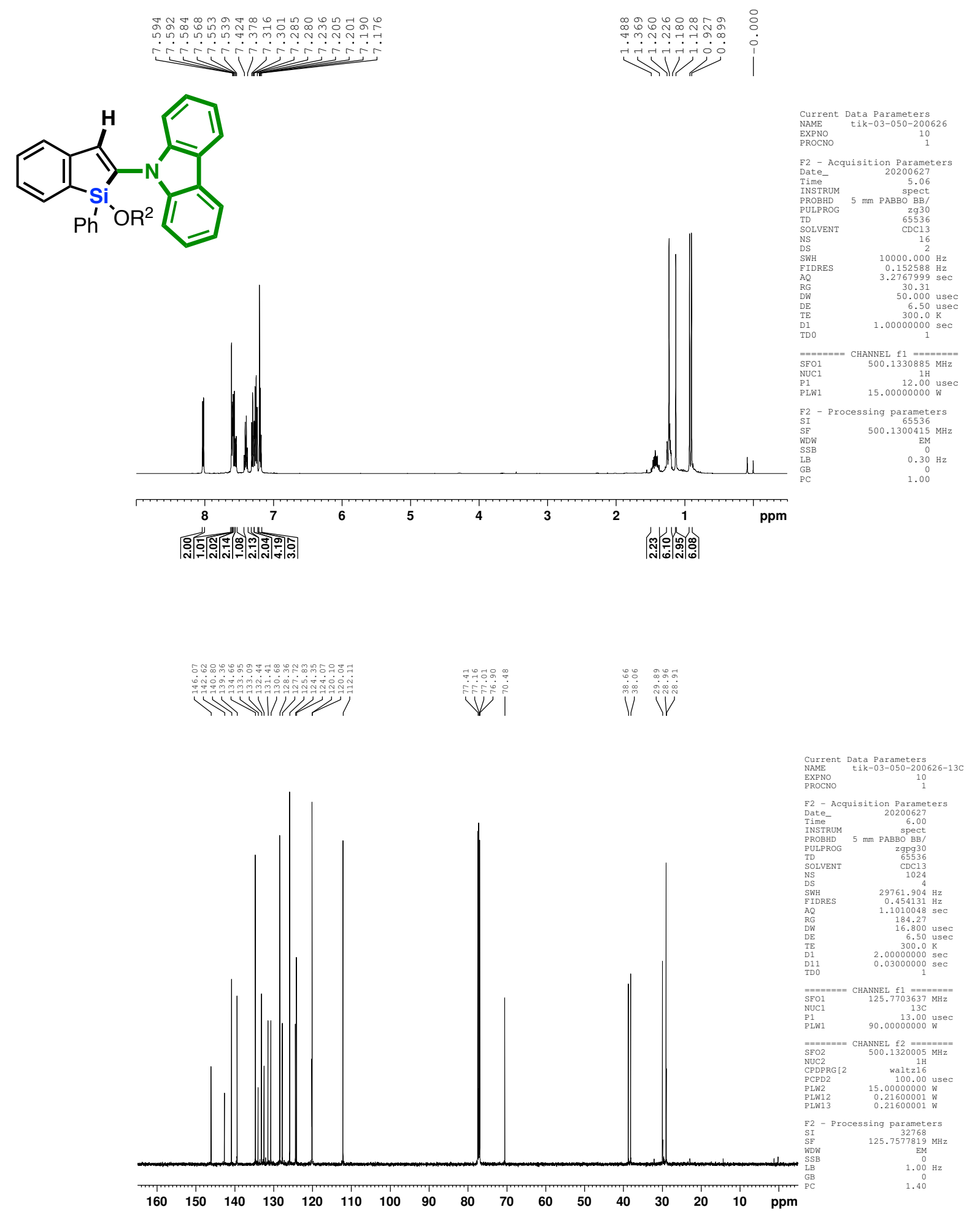




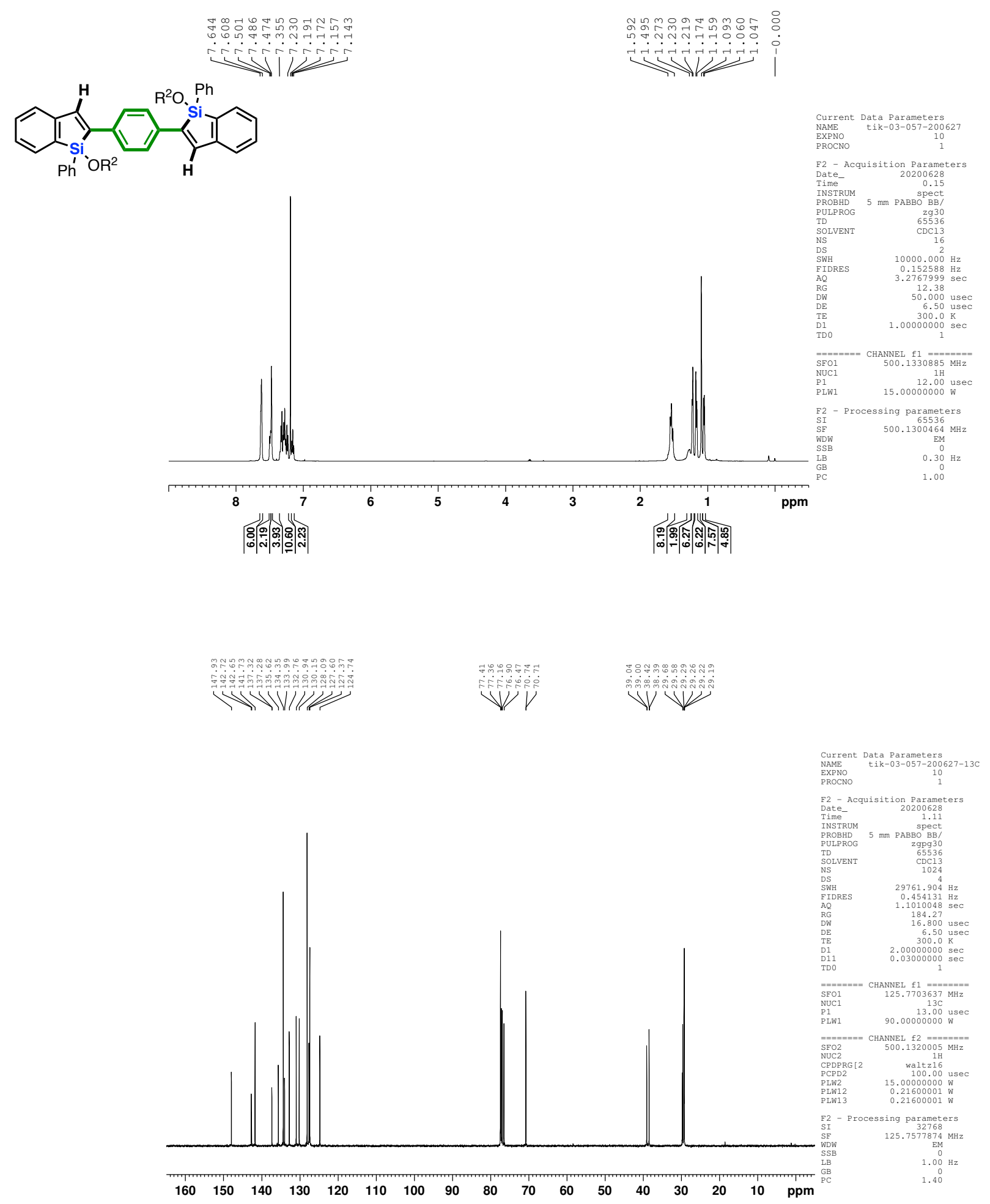




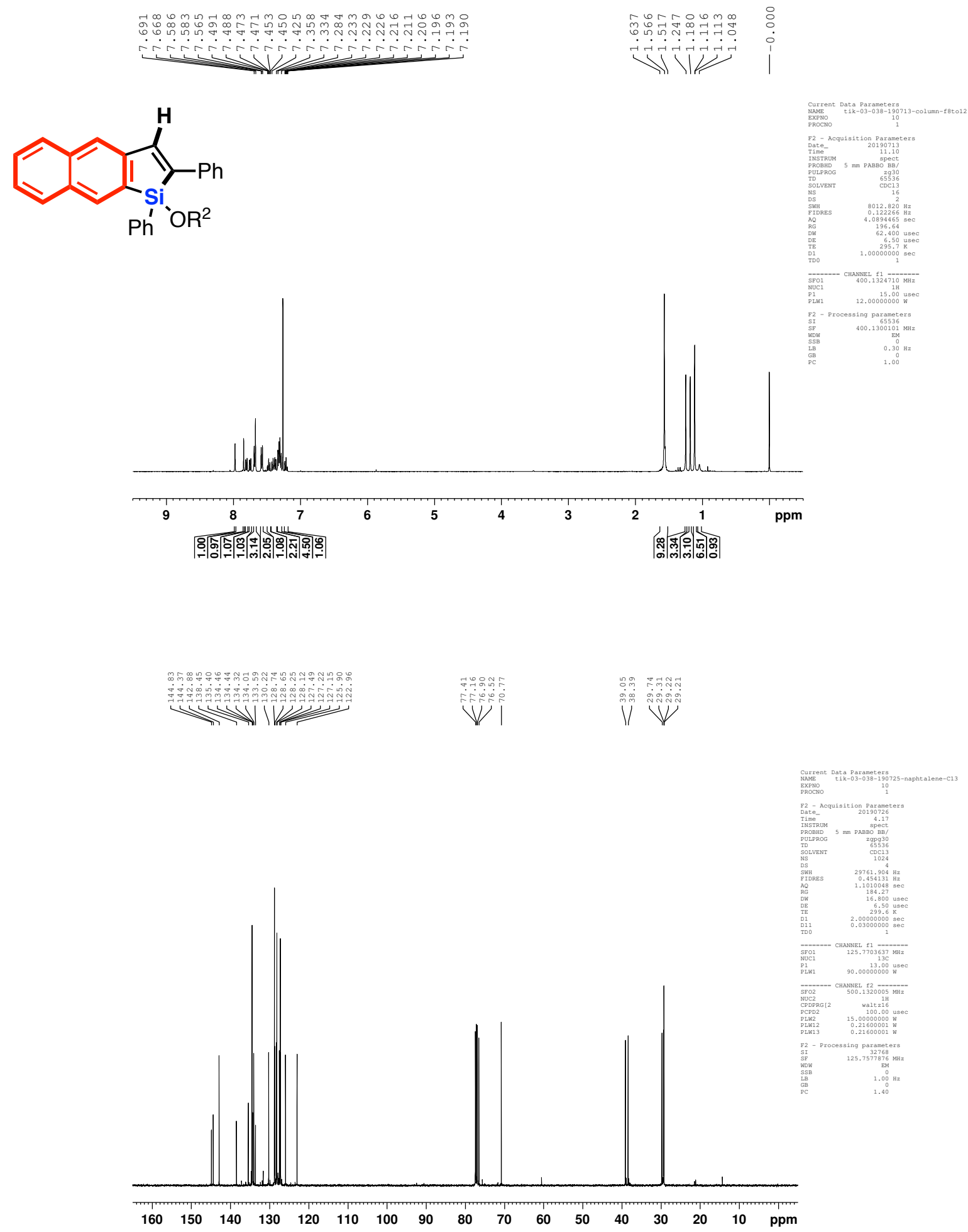



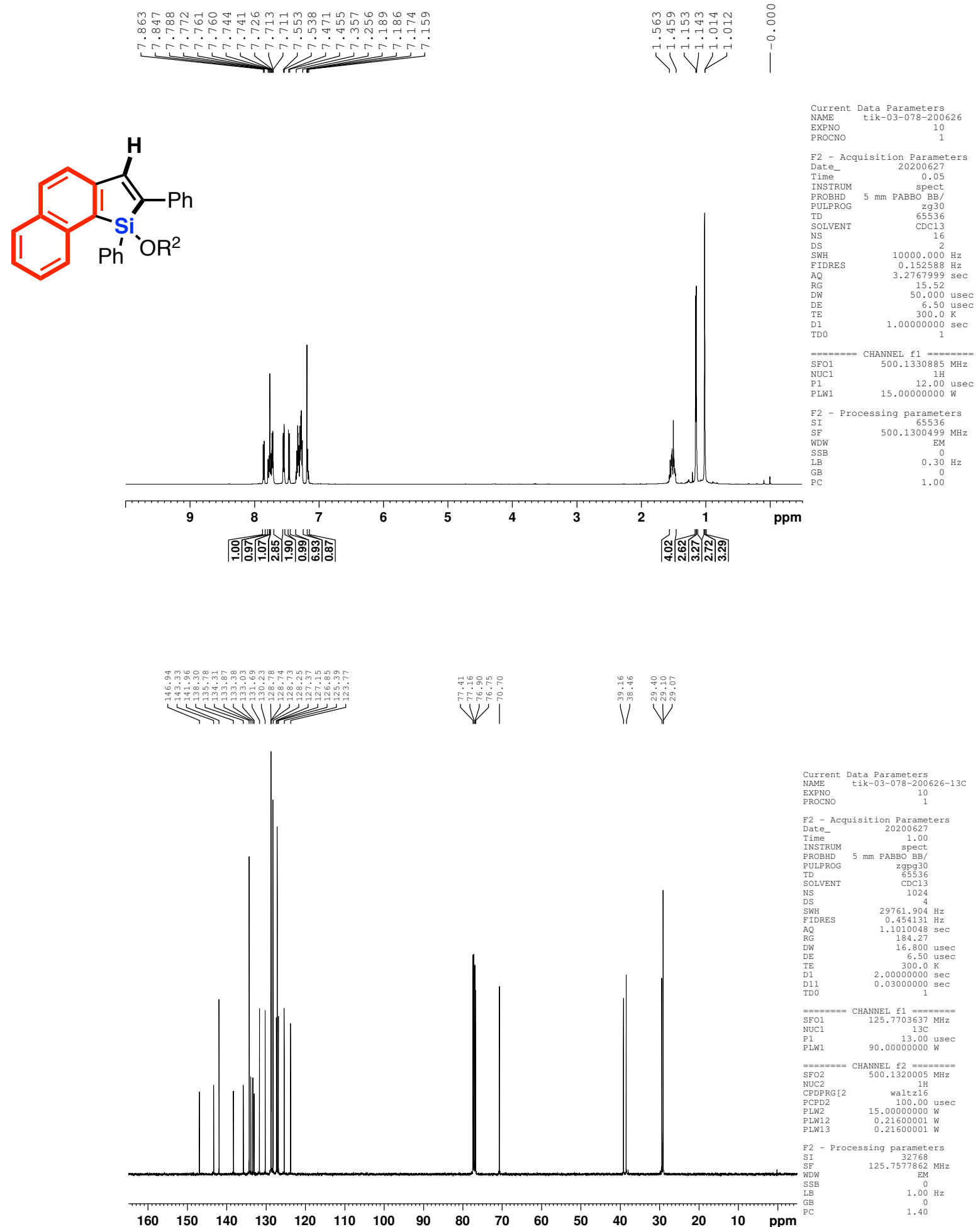


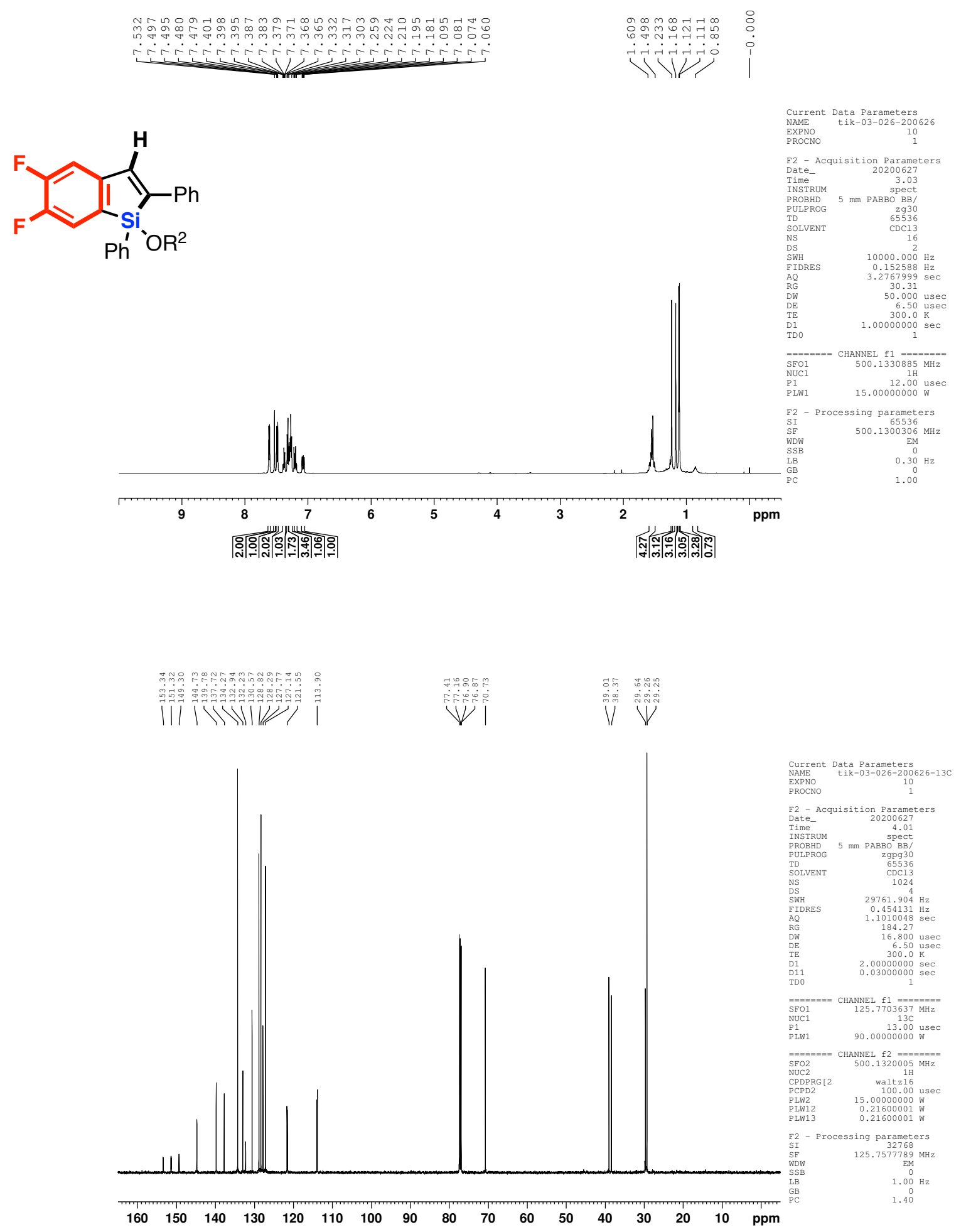




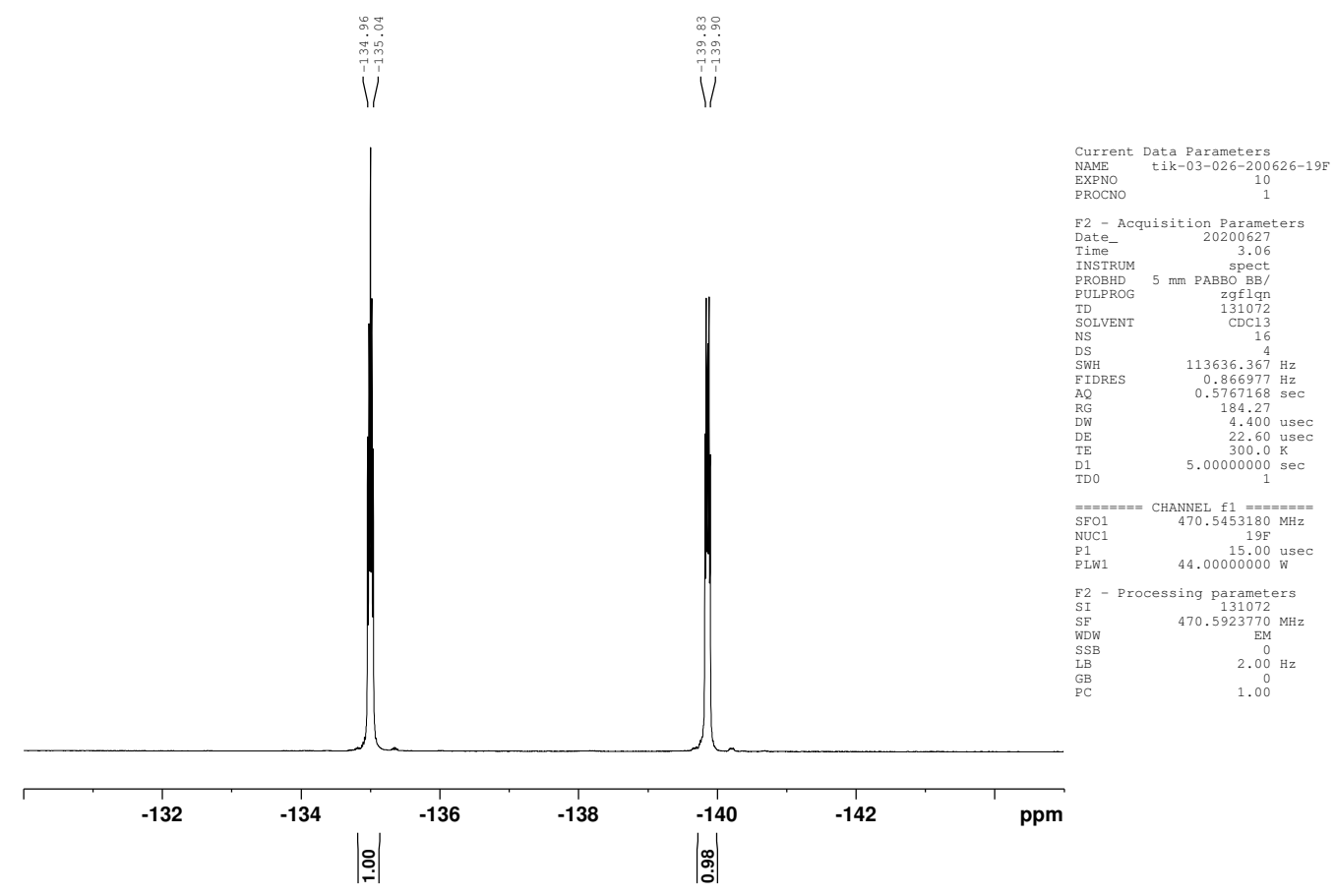



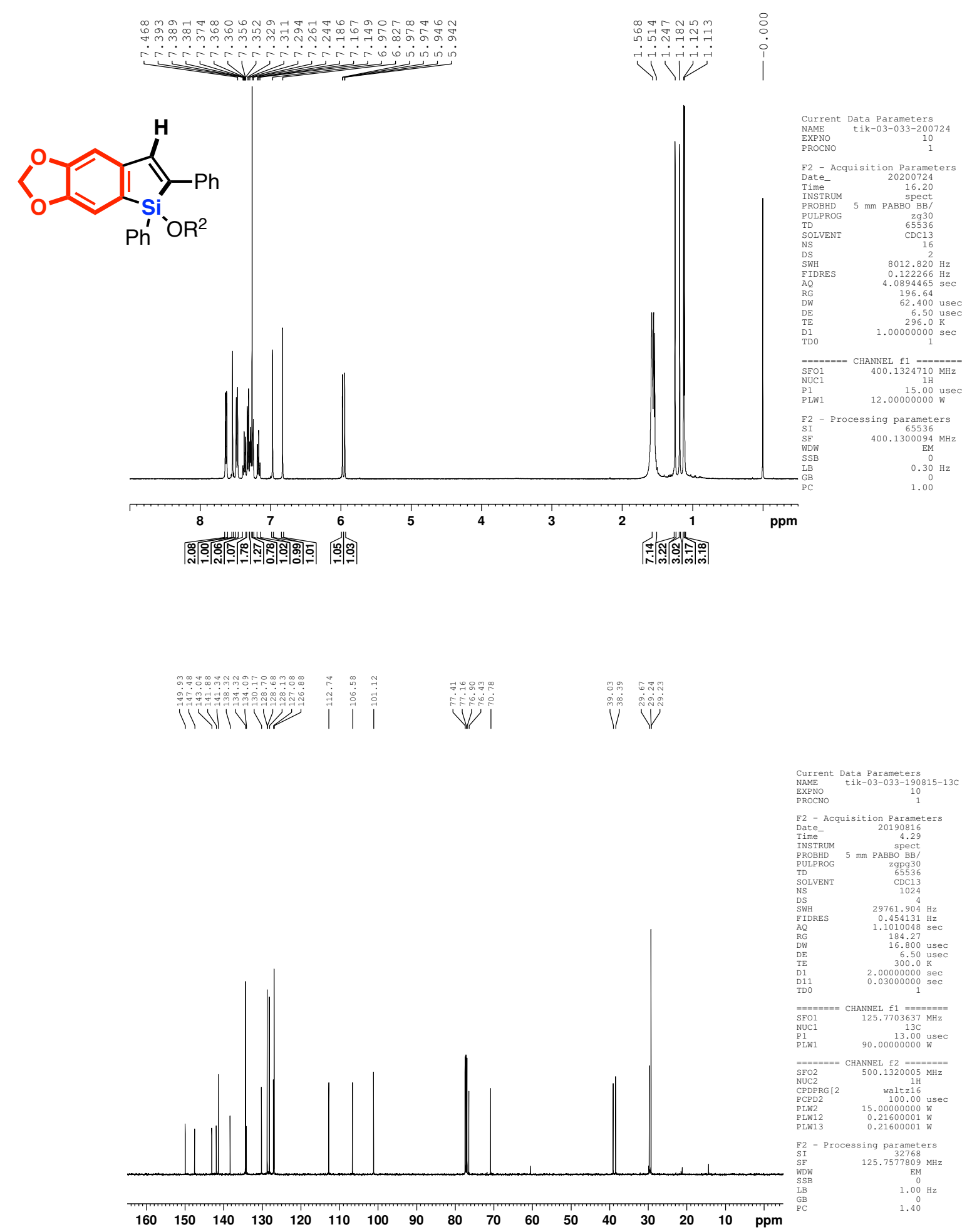

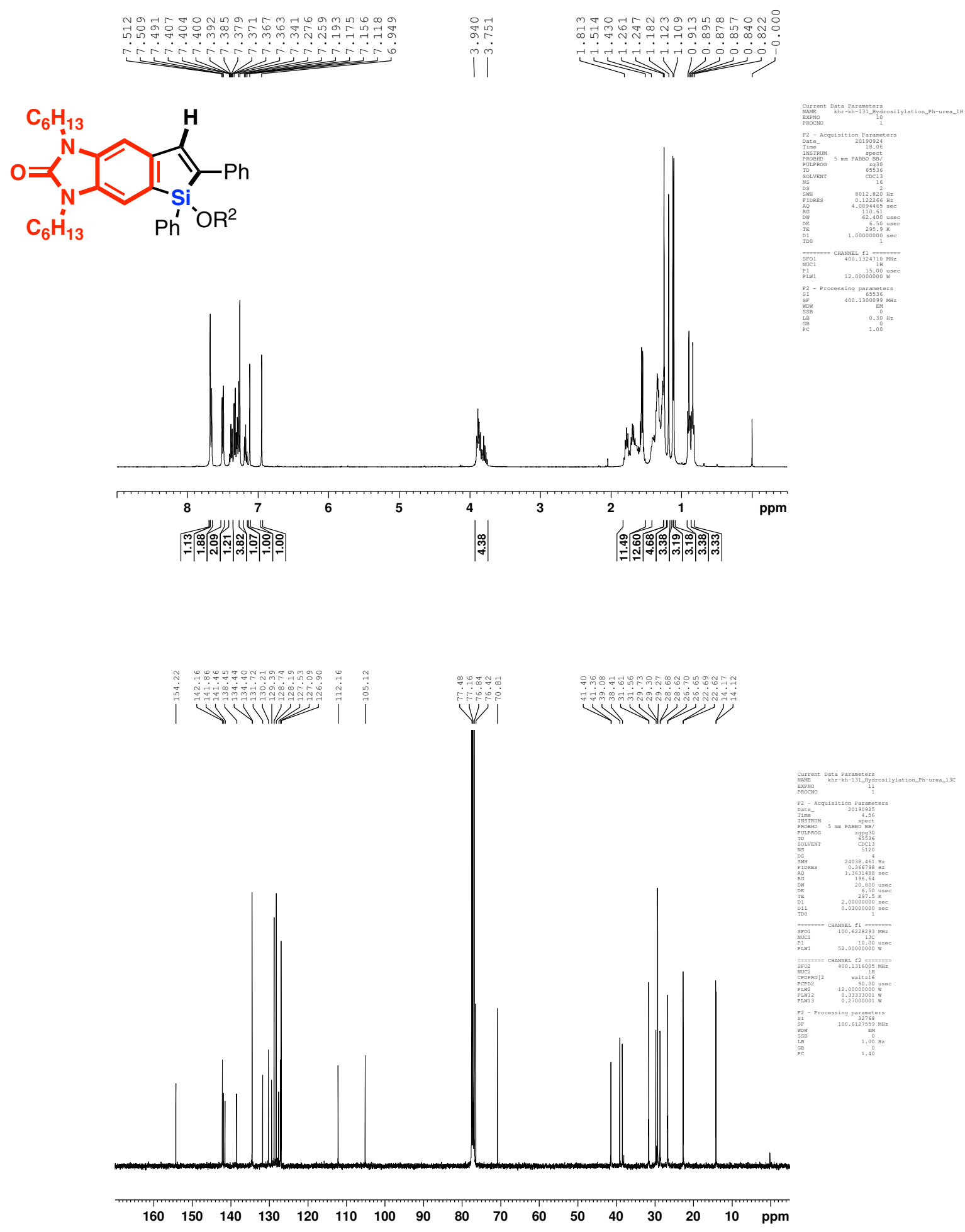

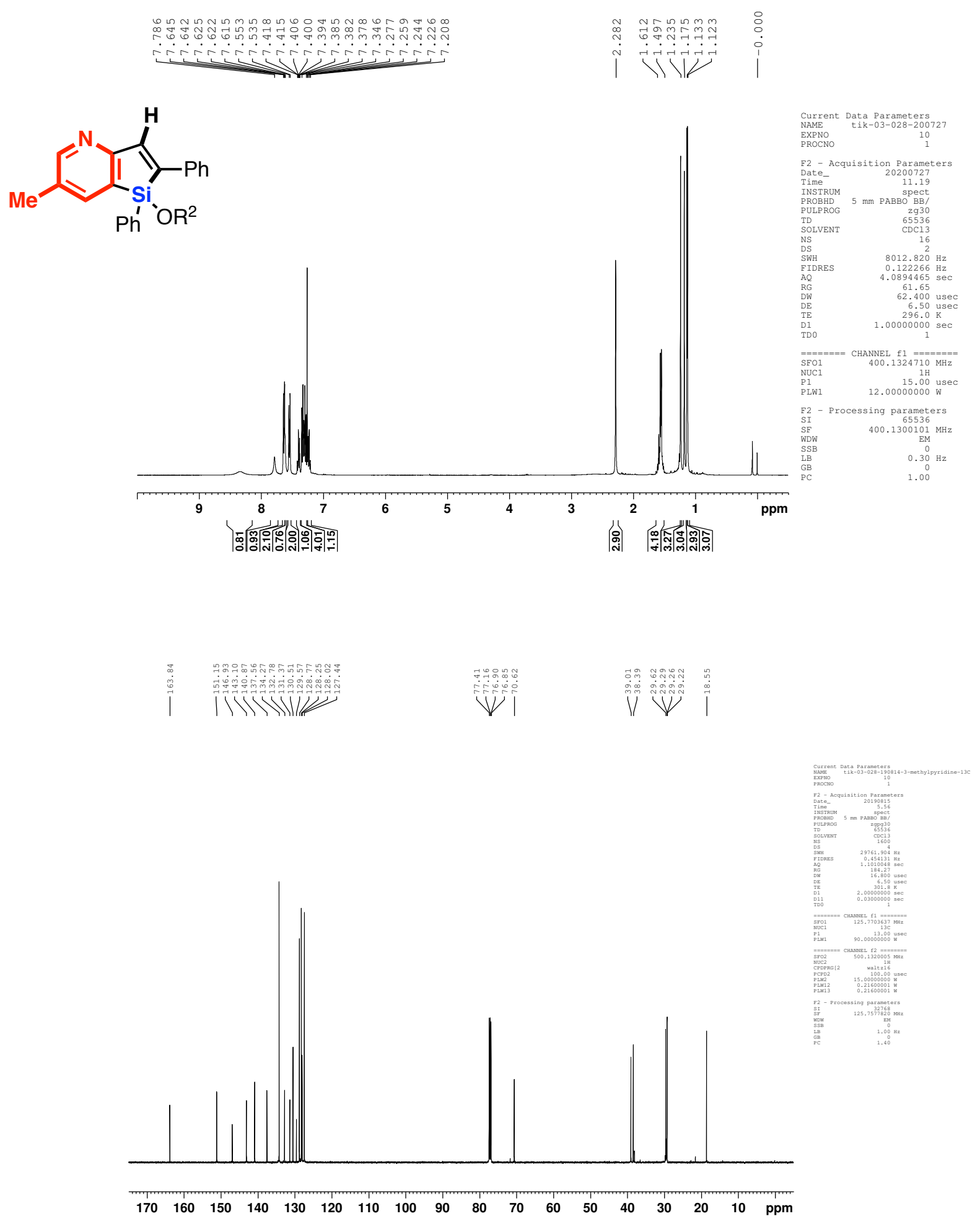


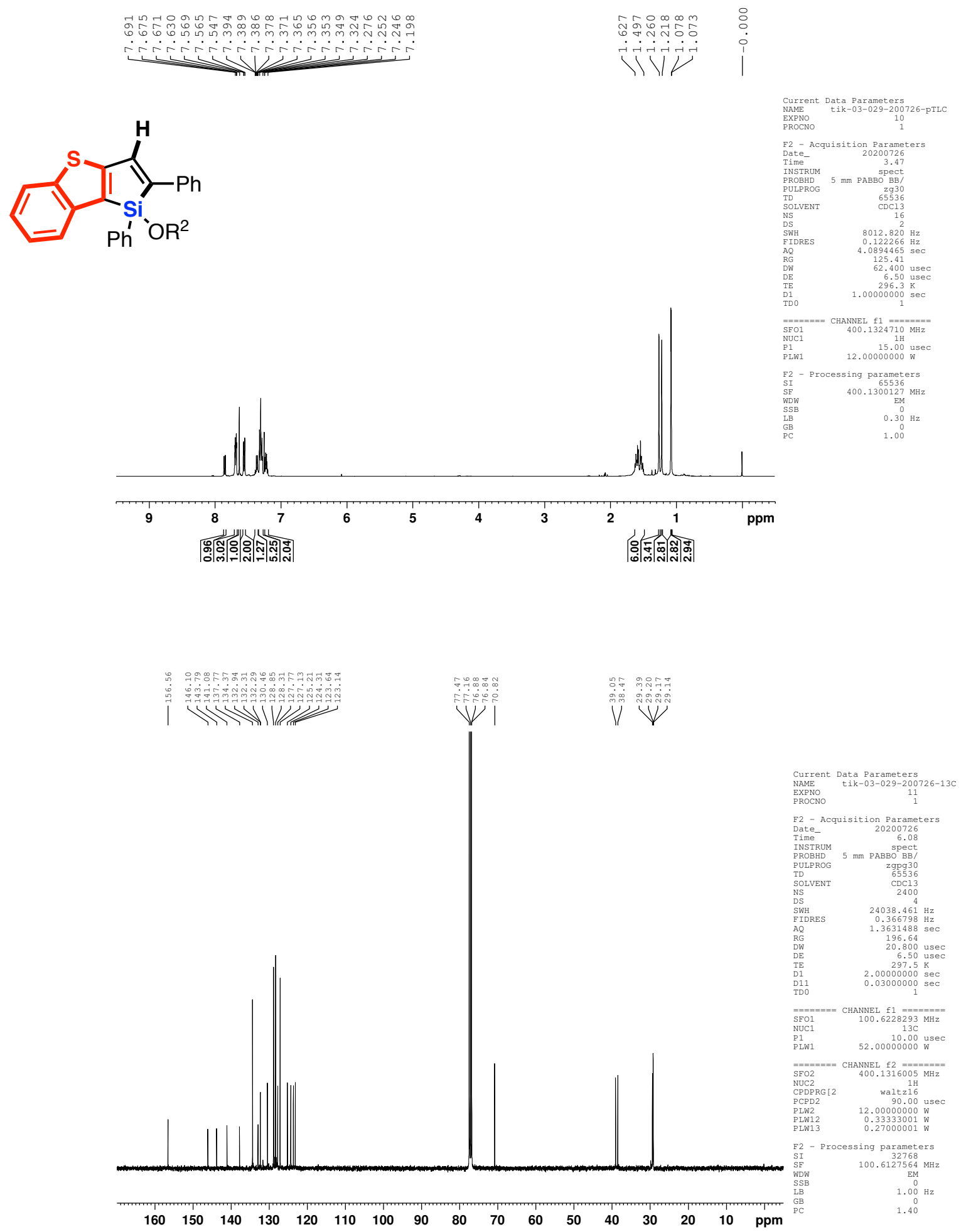




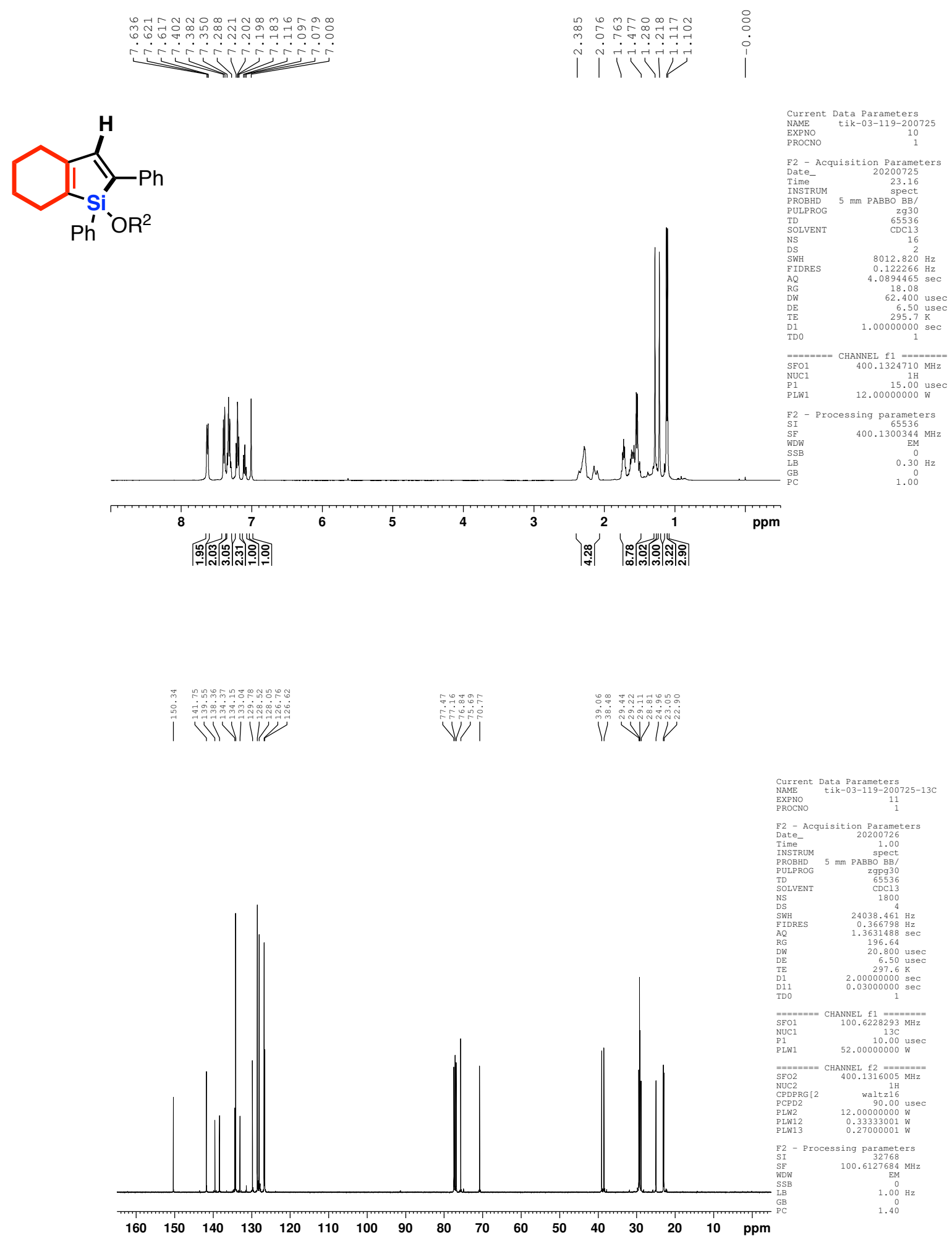



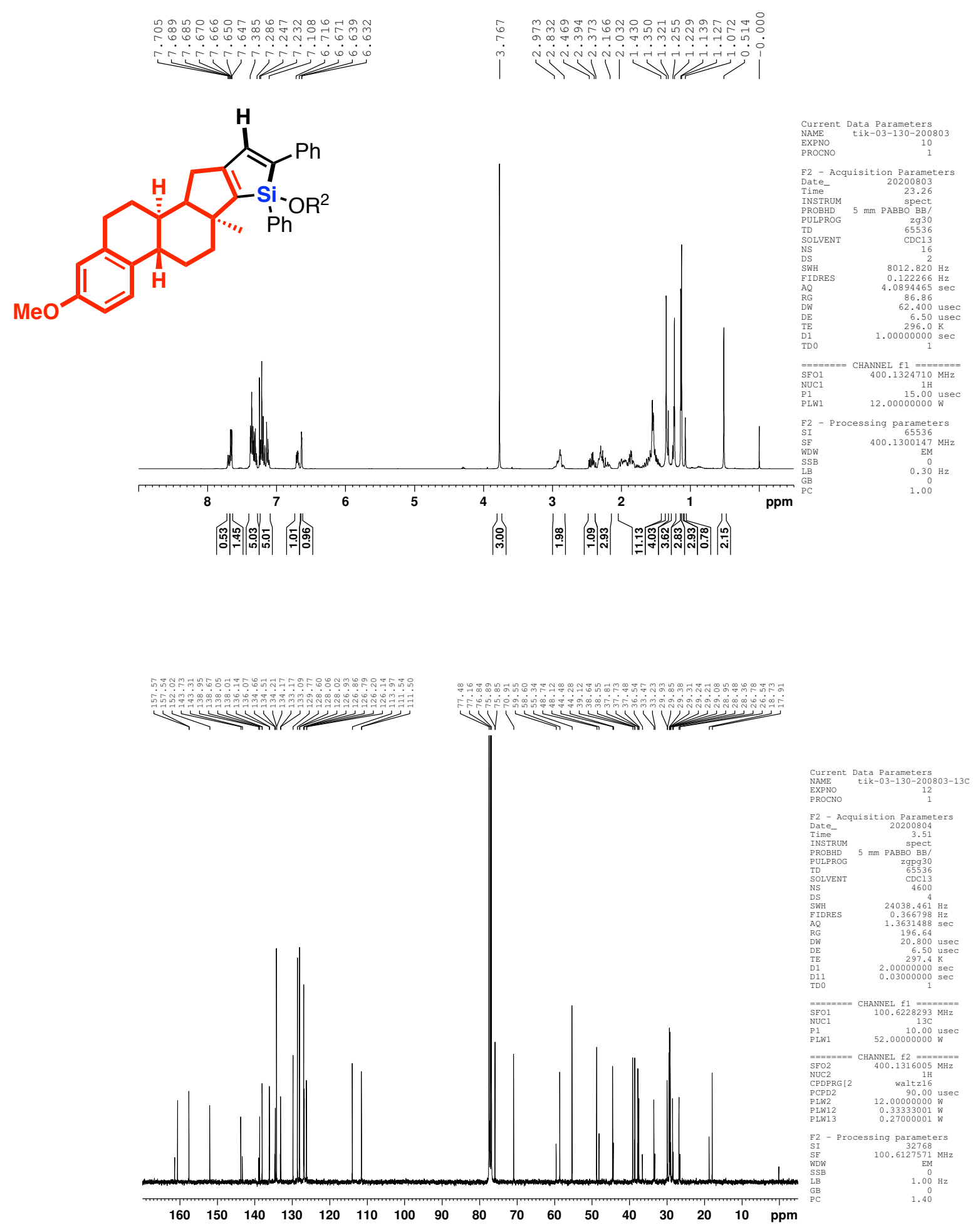

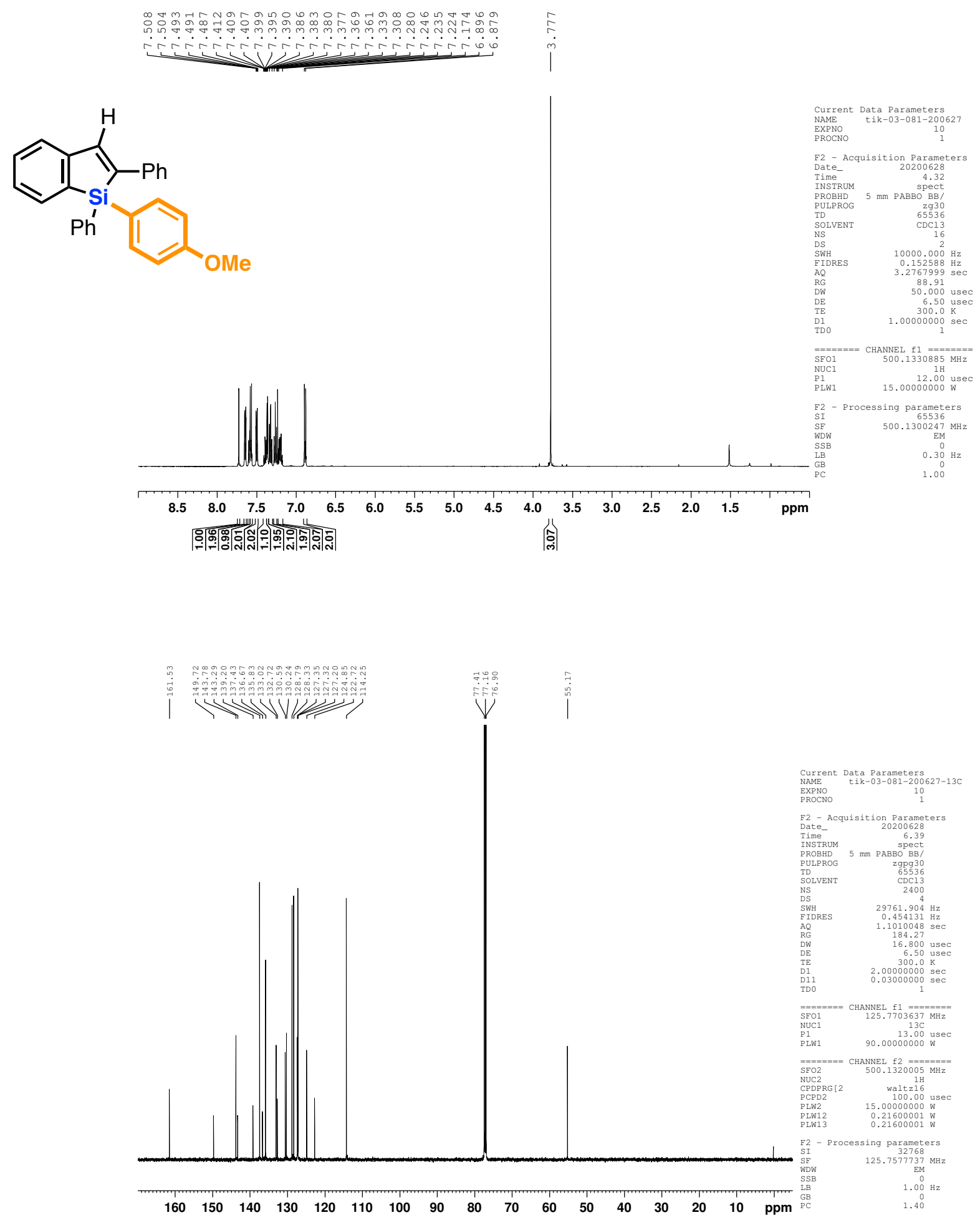


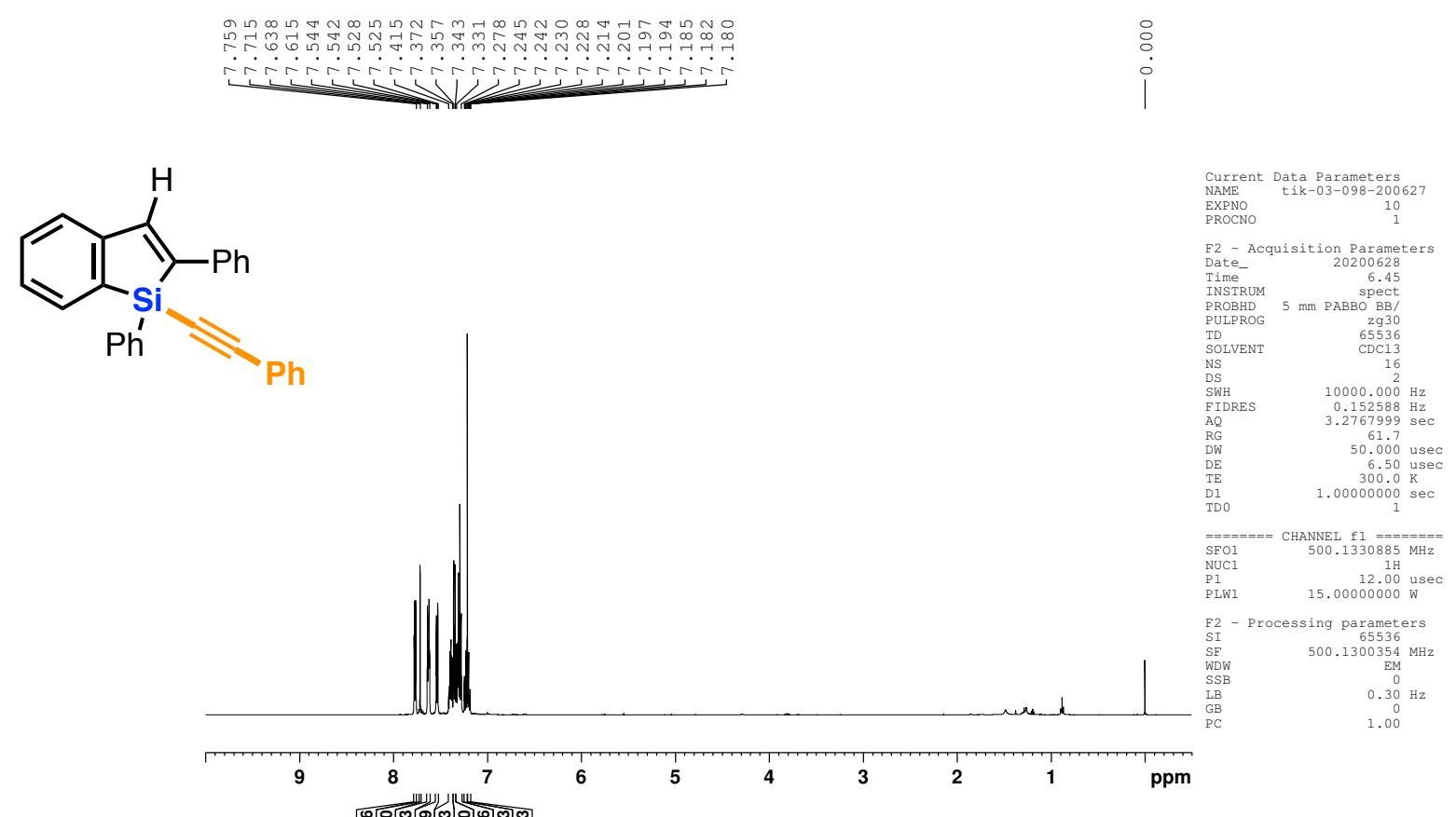
:
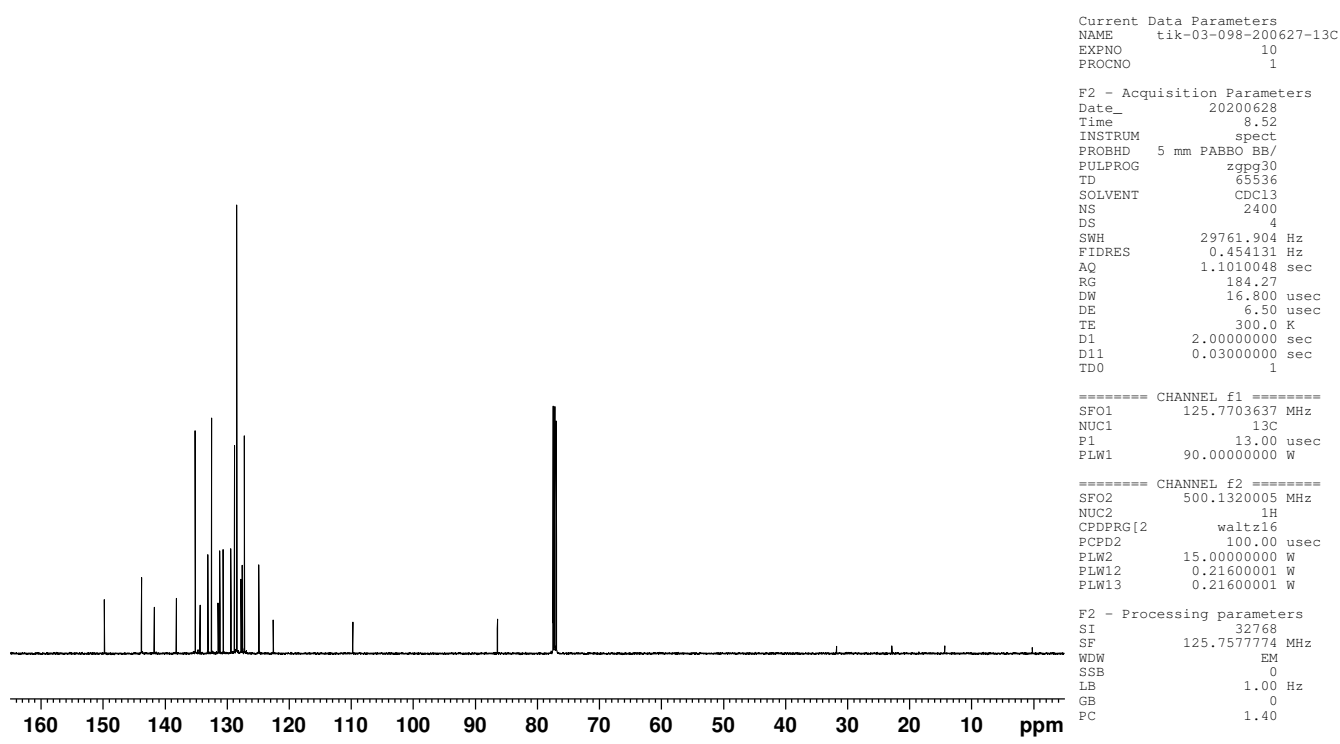

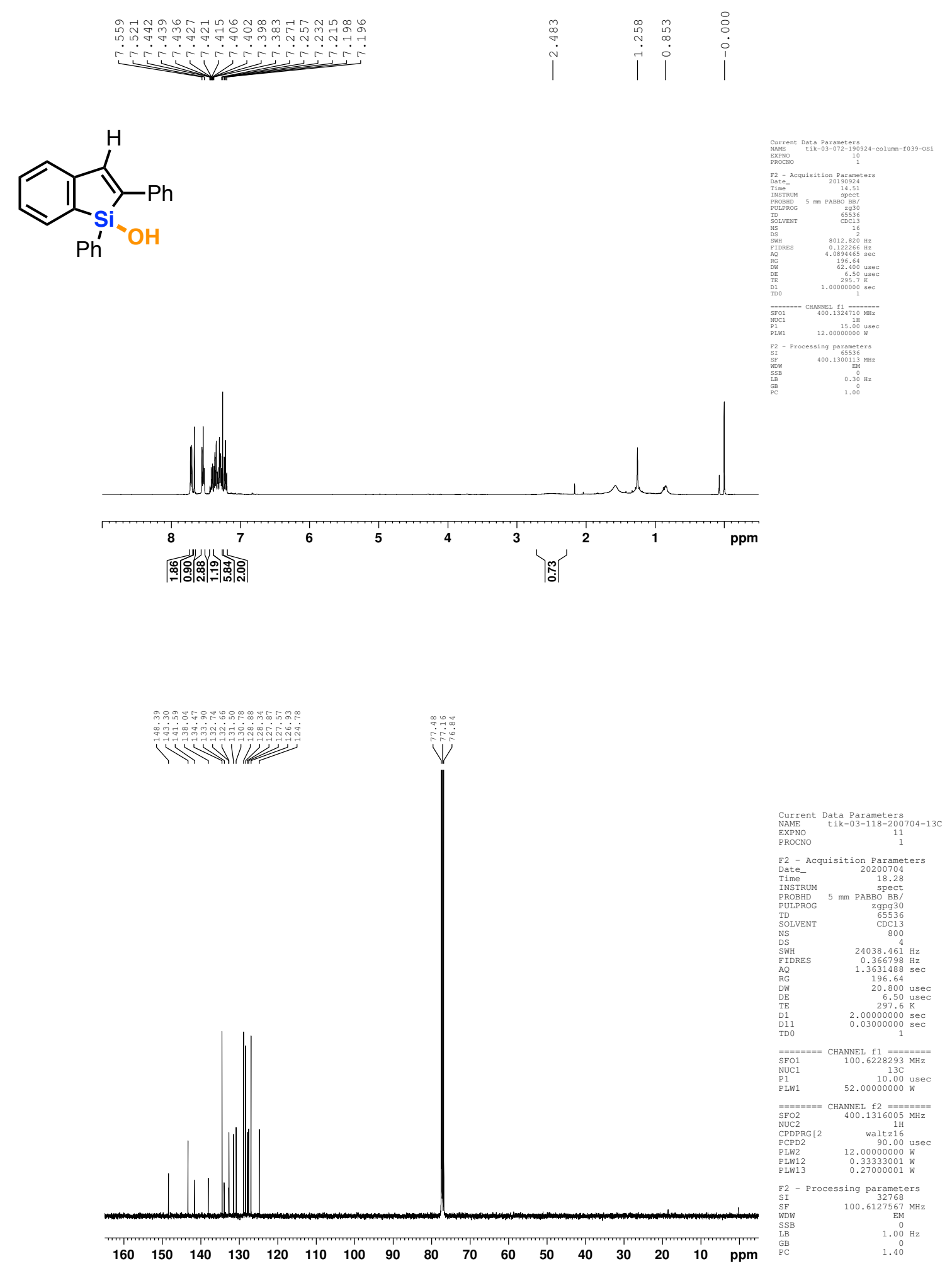


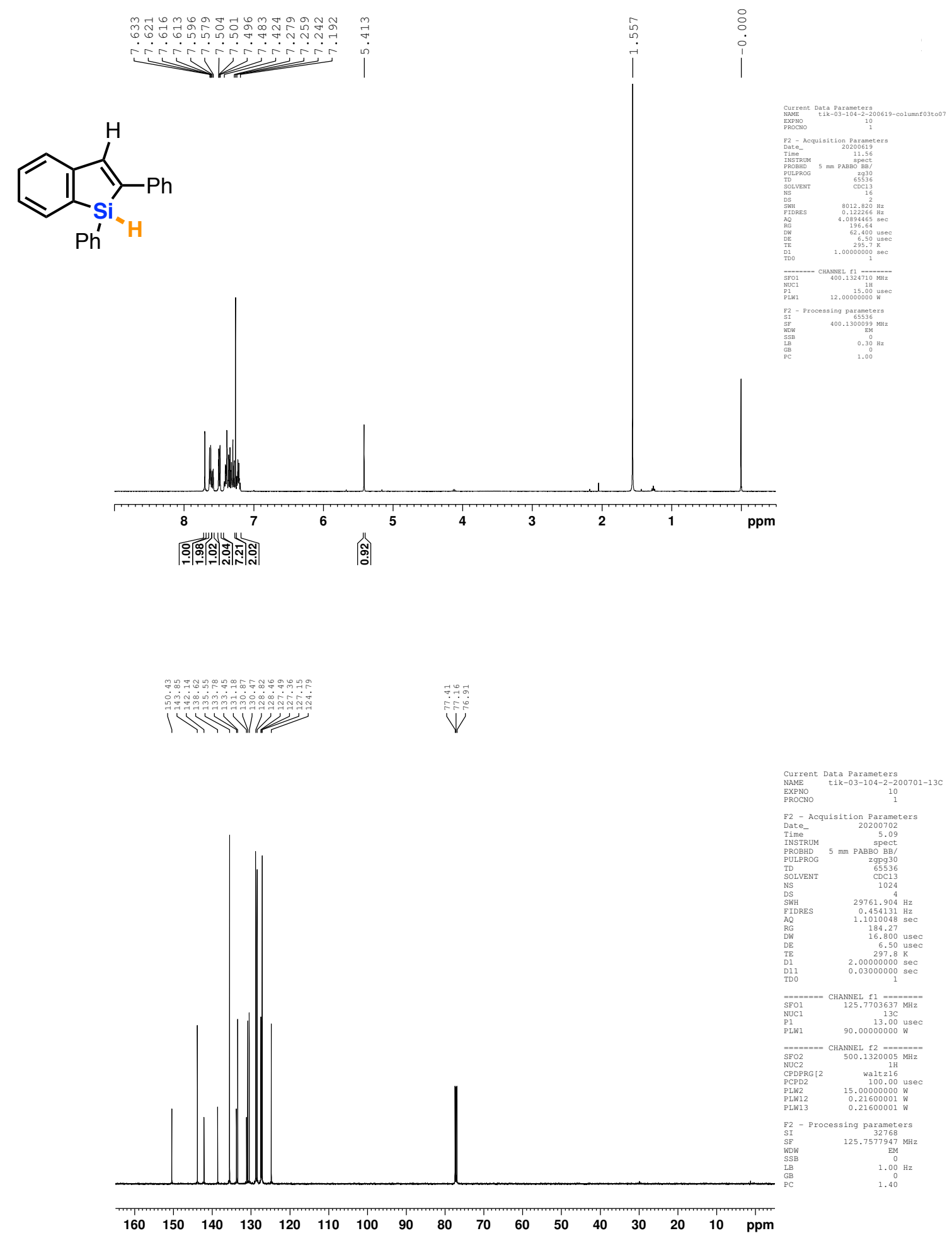




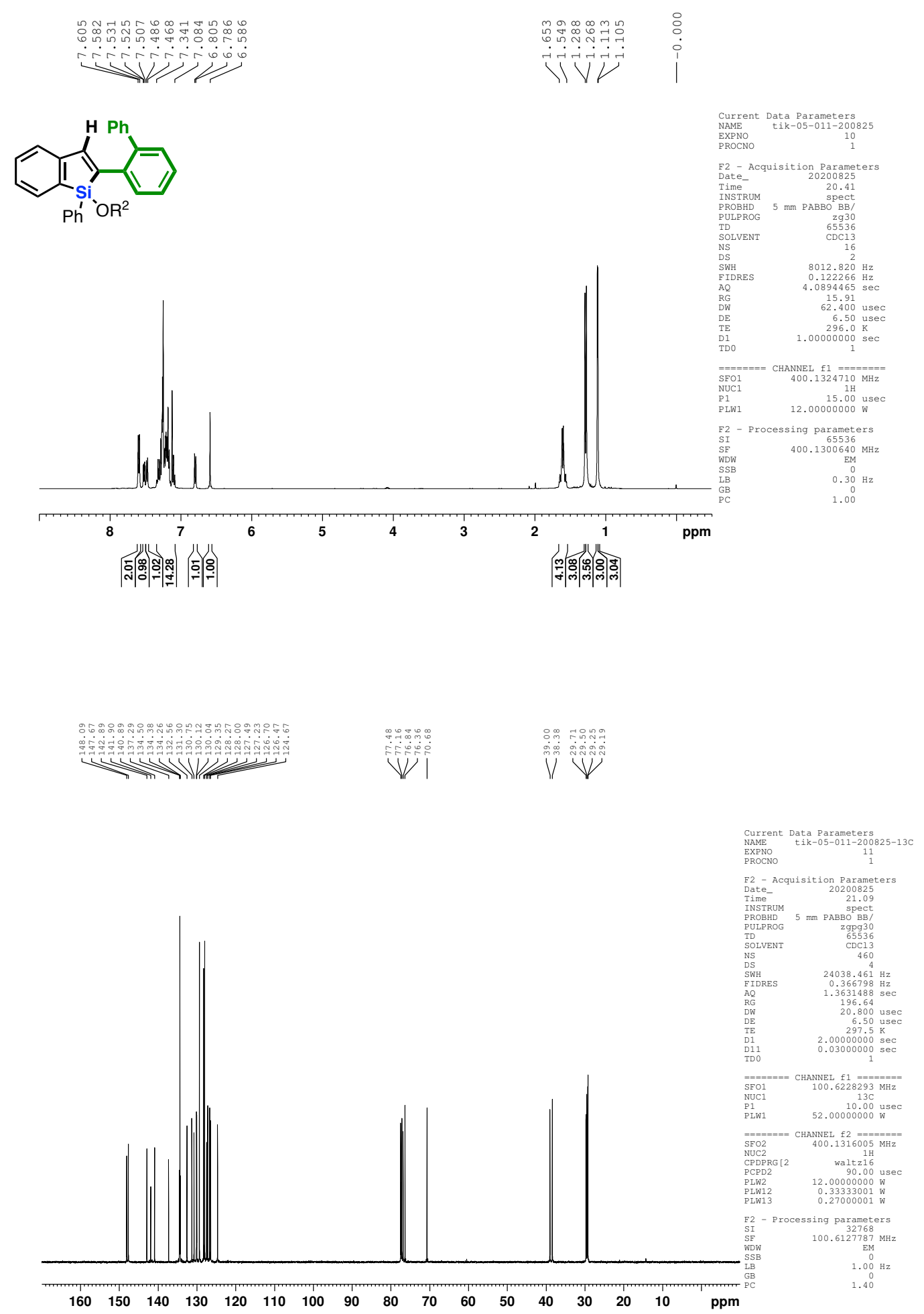



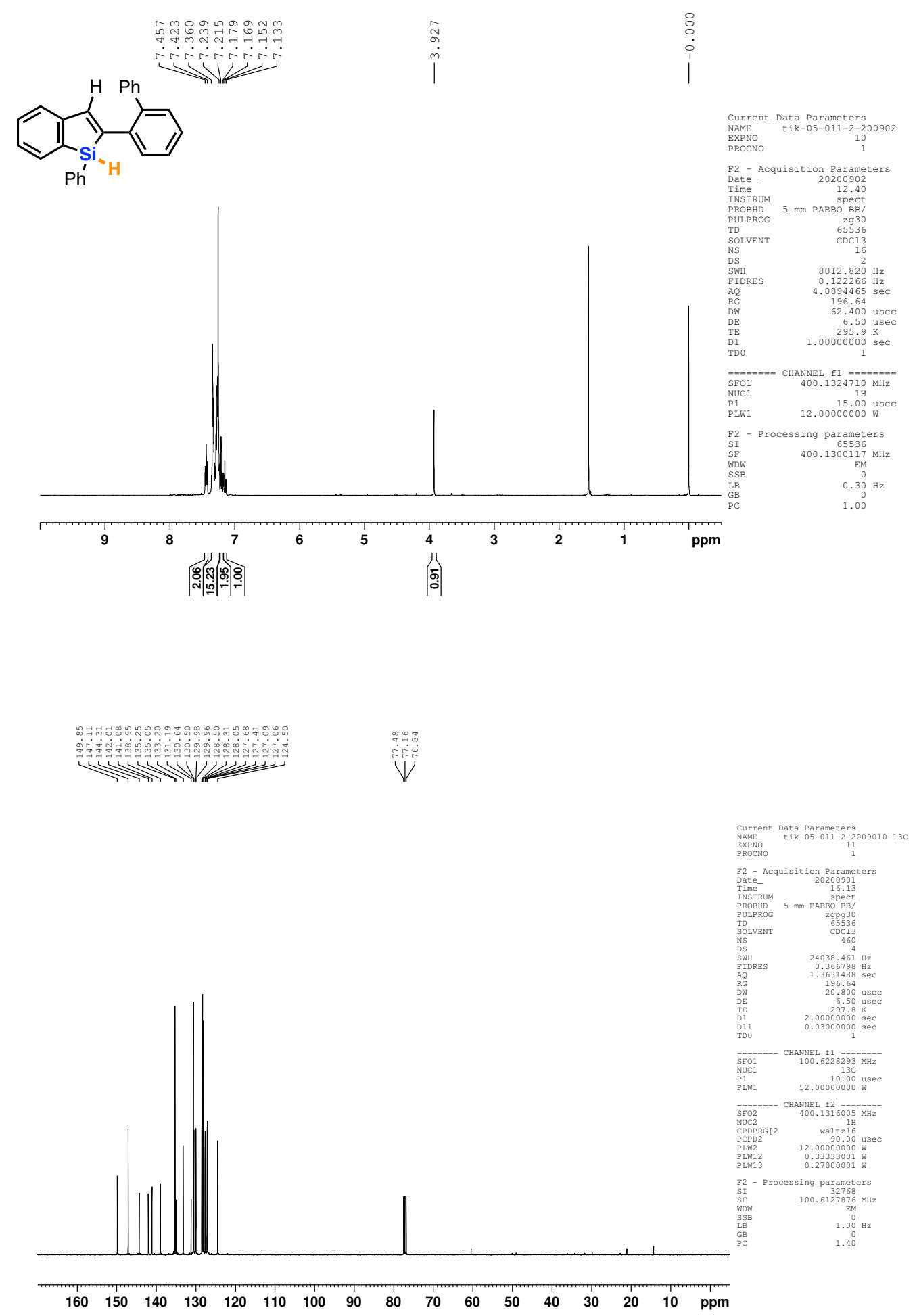
9-phenyl-9H-dibenzo[c,e]benzo[4,5] silolo[1,2-a]siline (10)
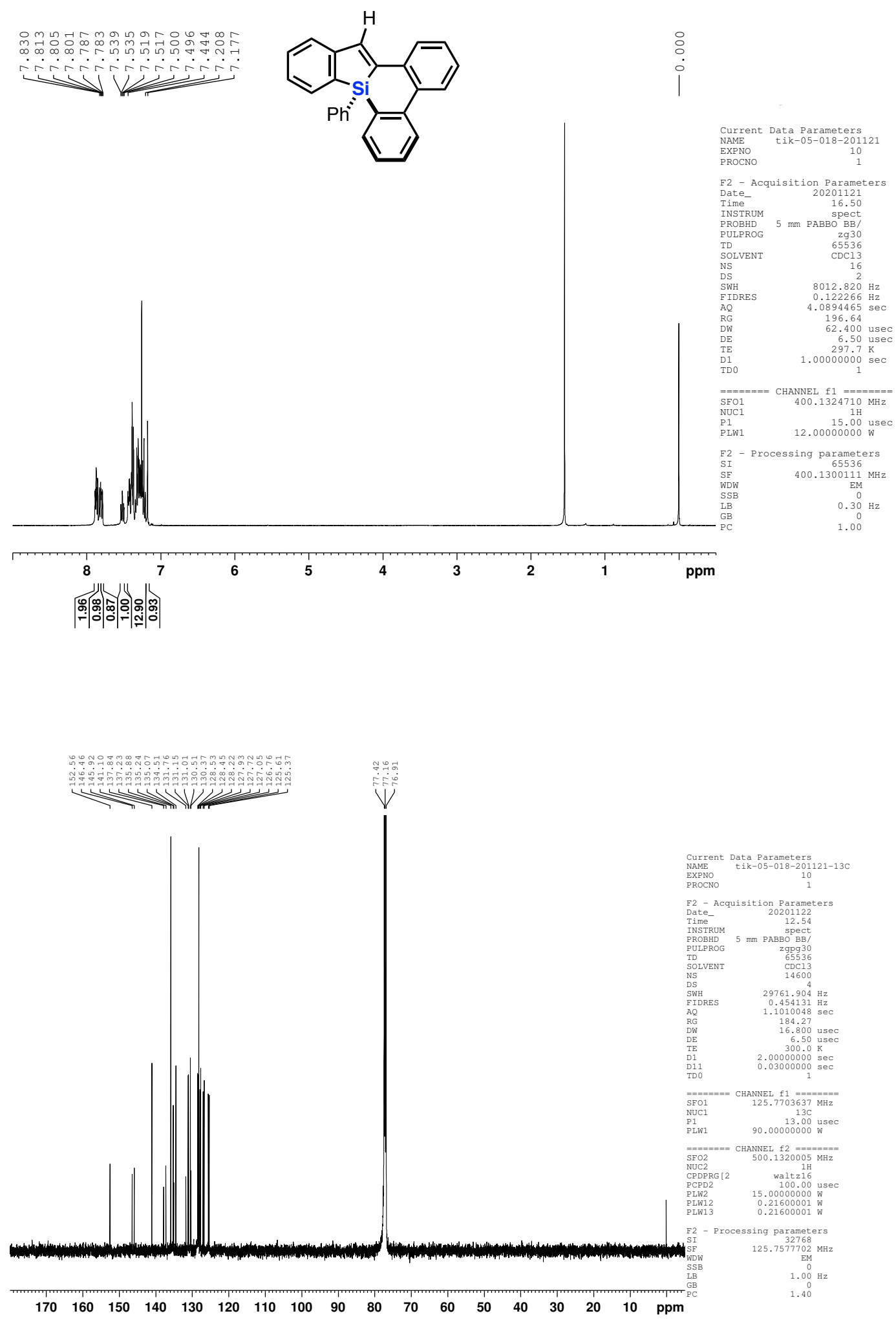


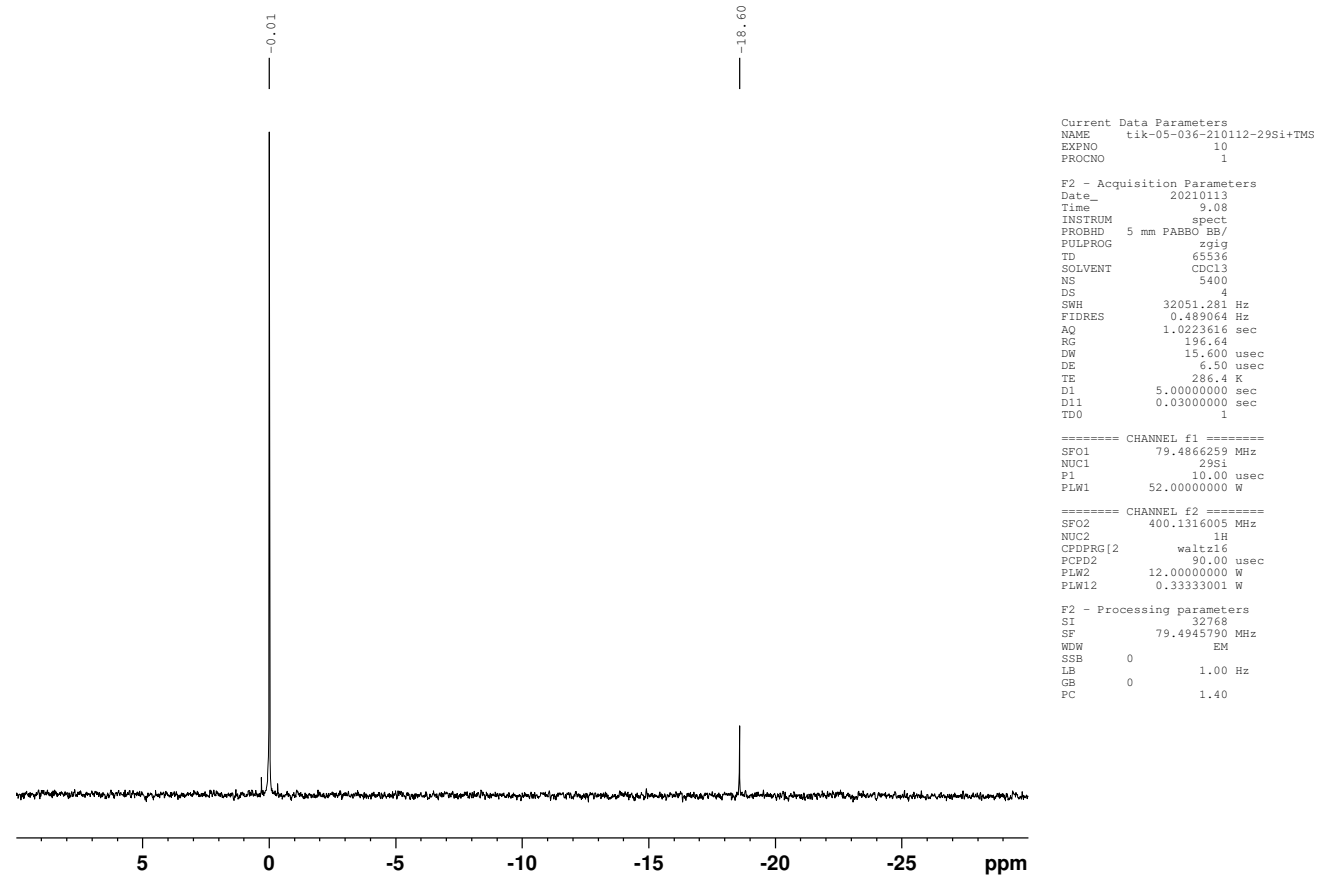




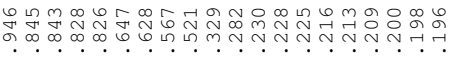

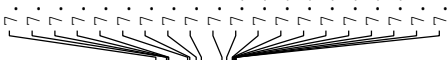
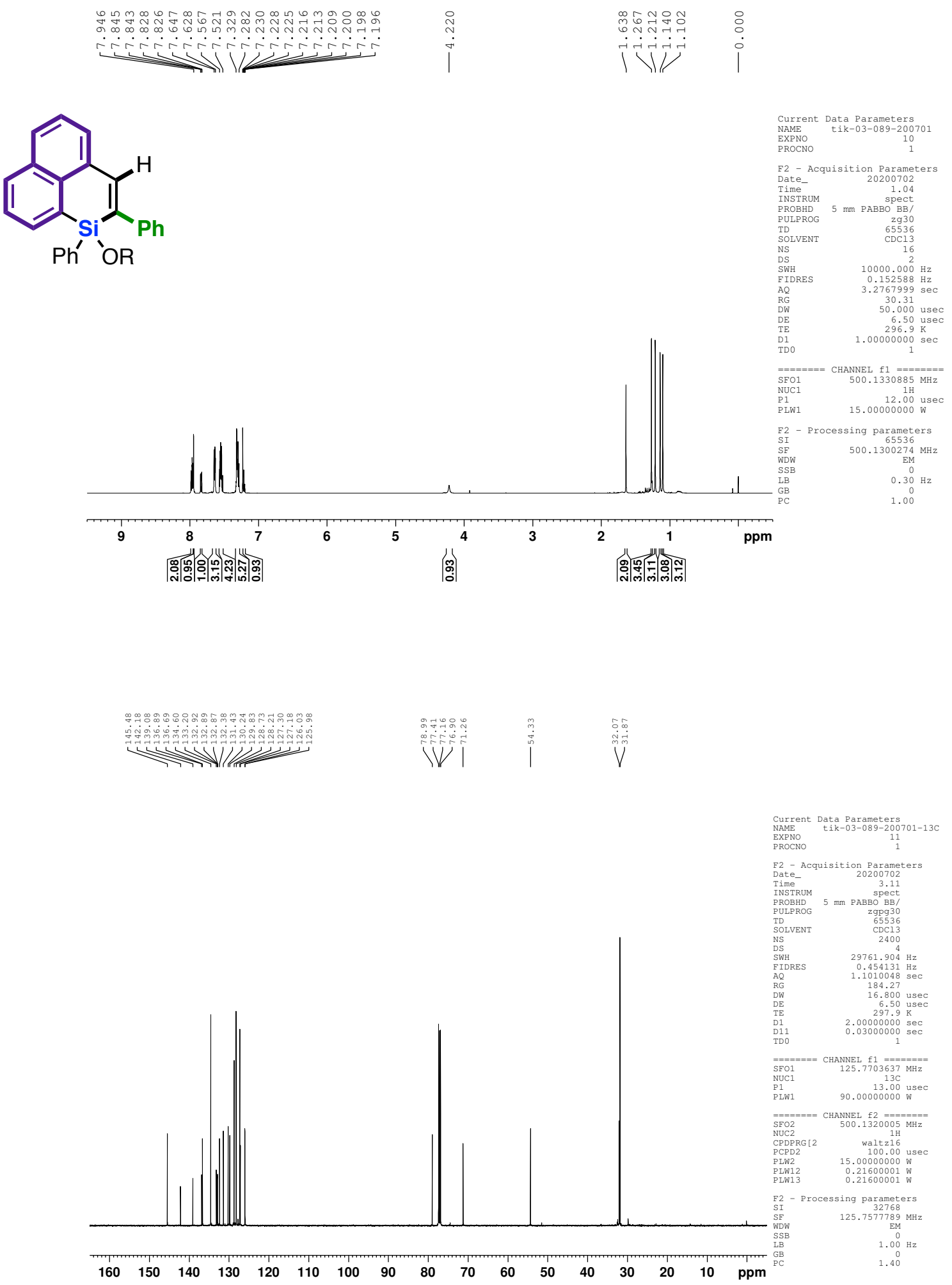


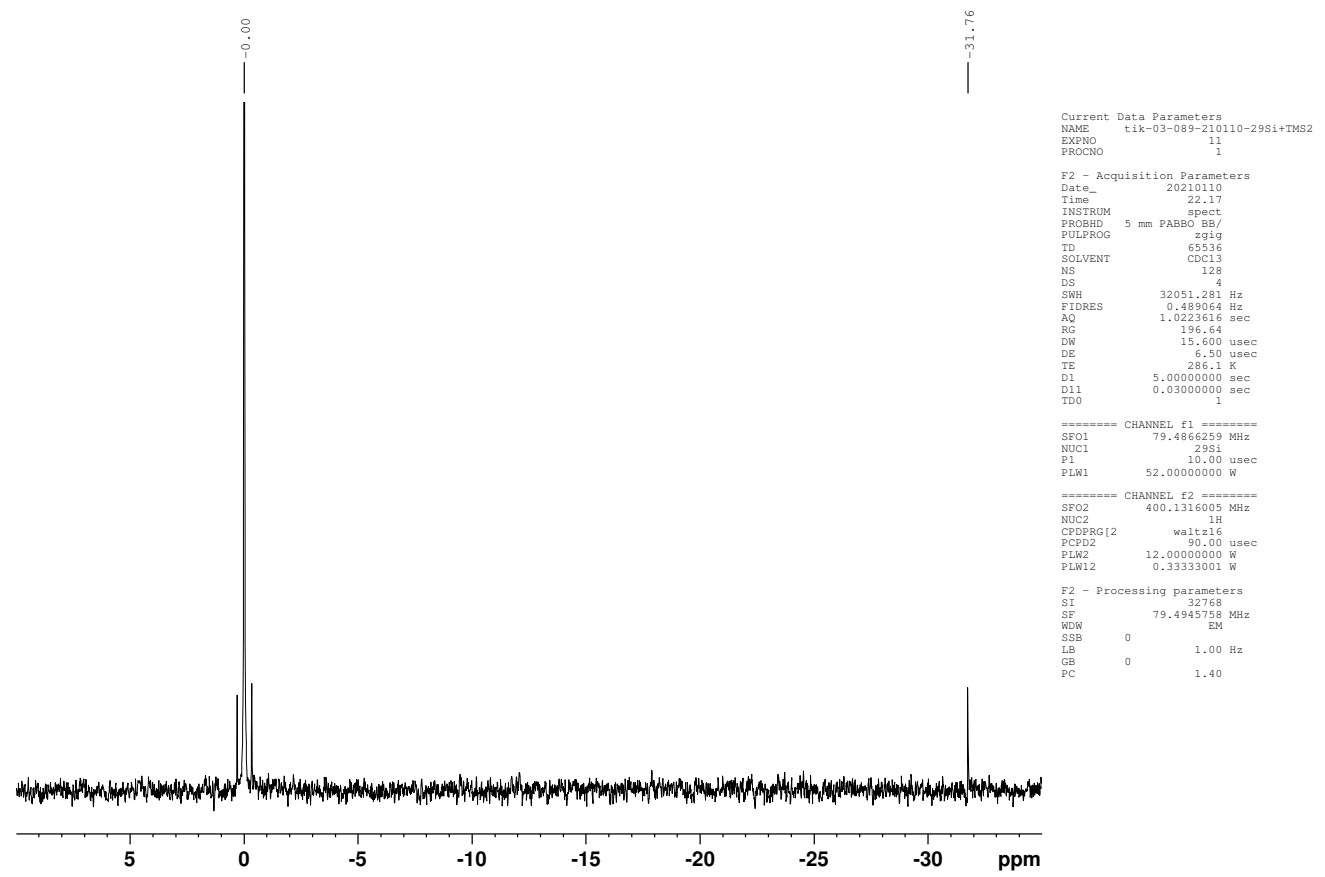




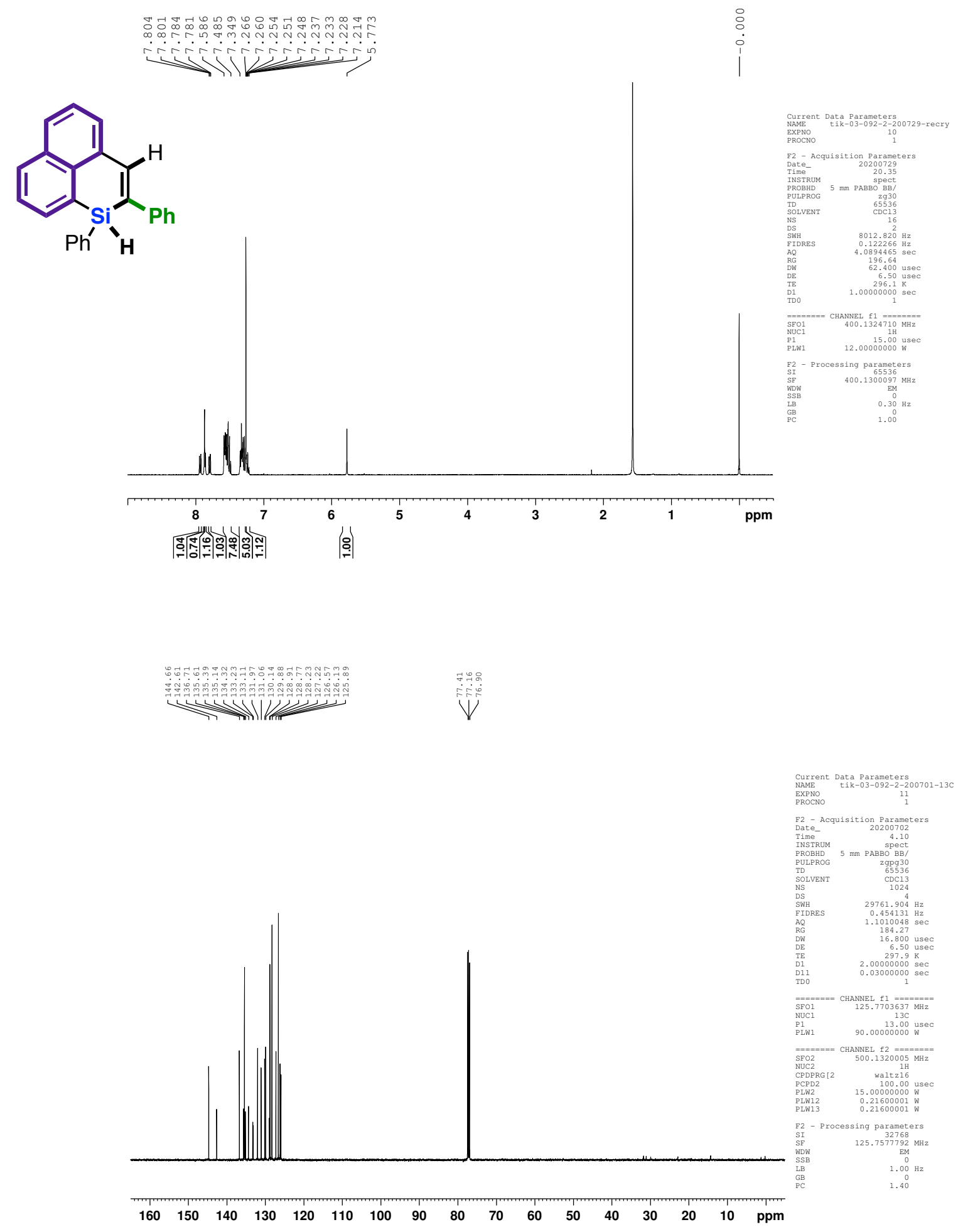



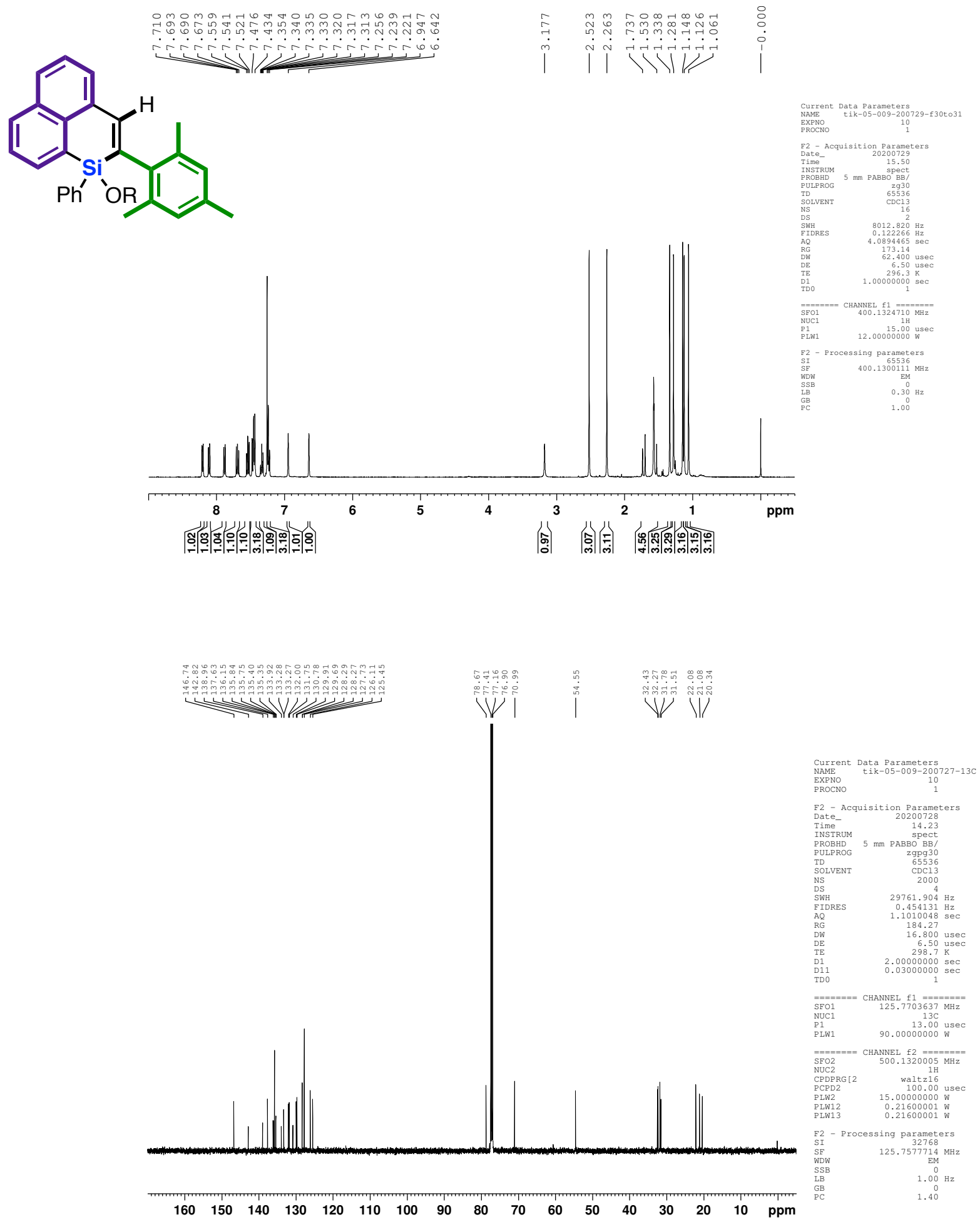

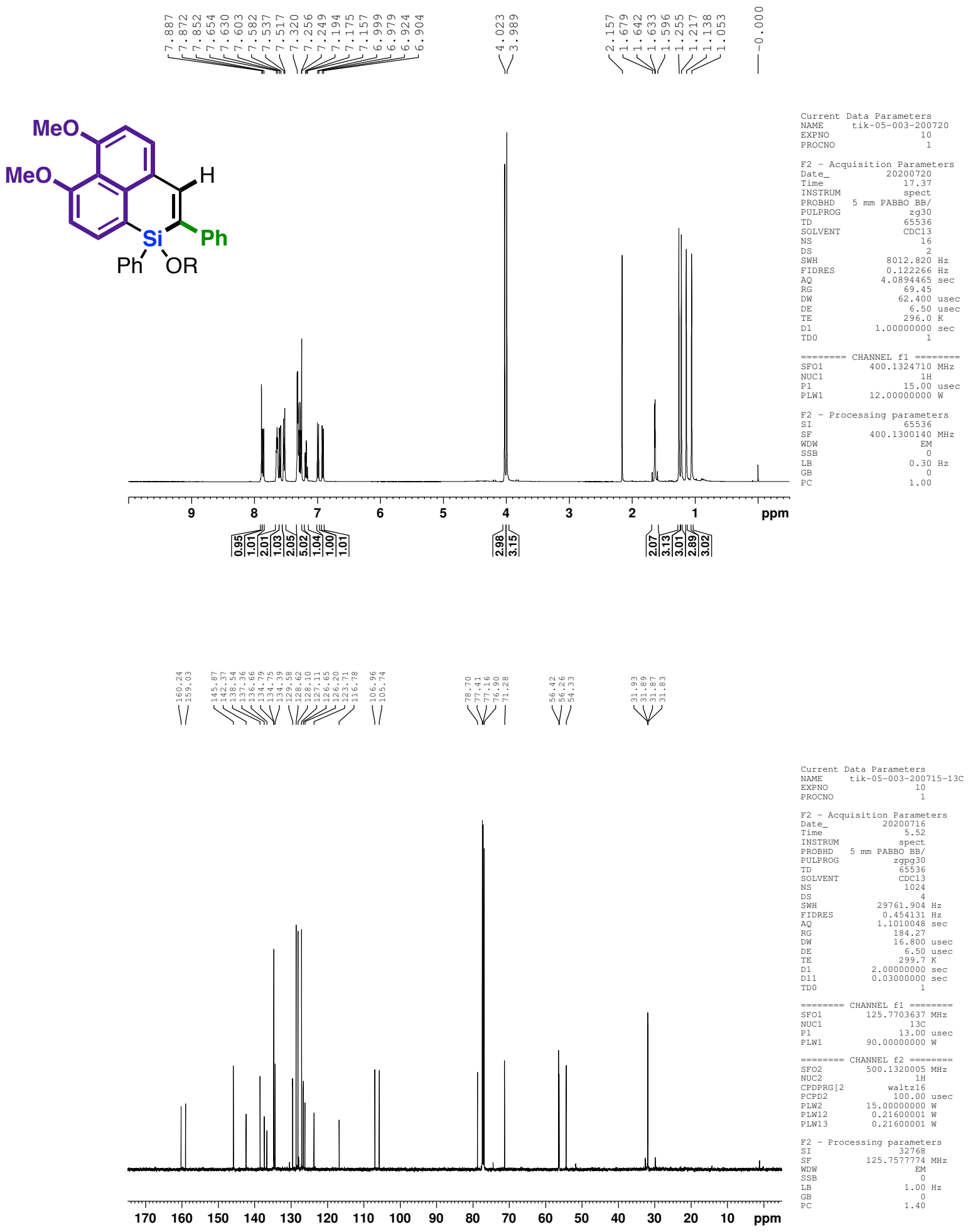


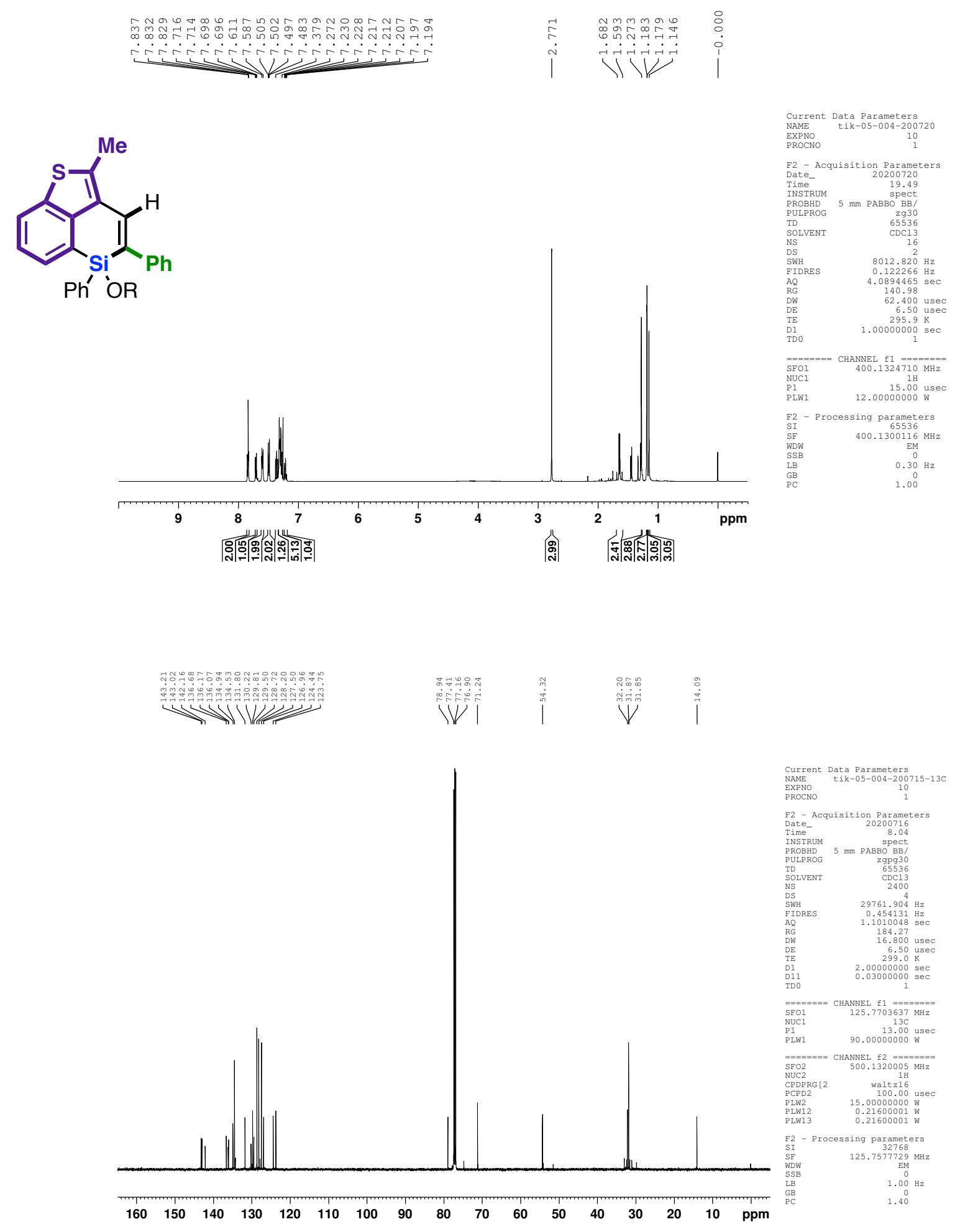



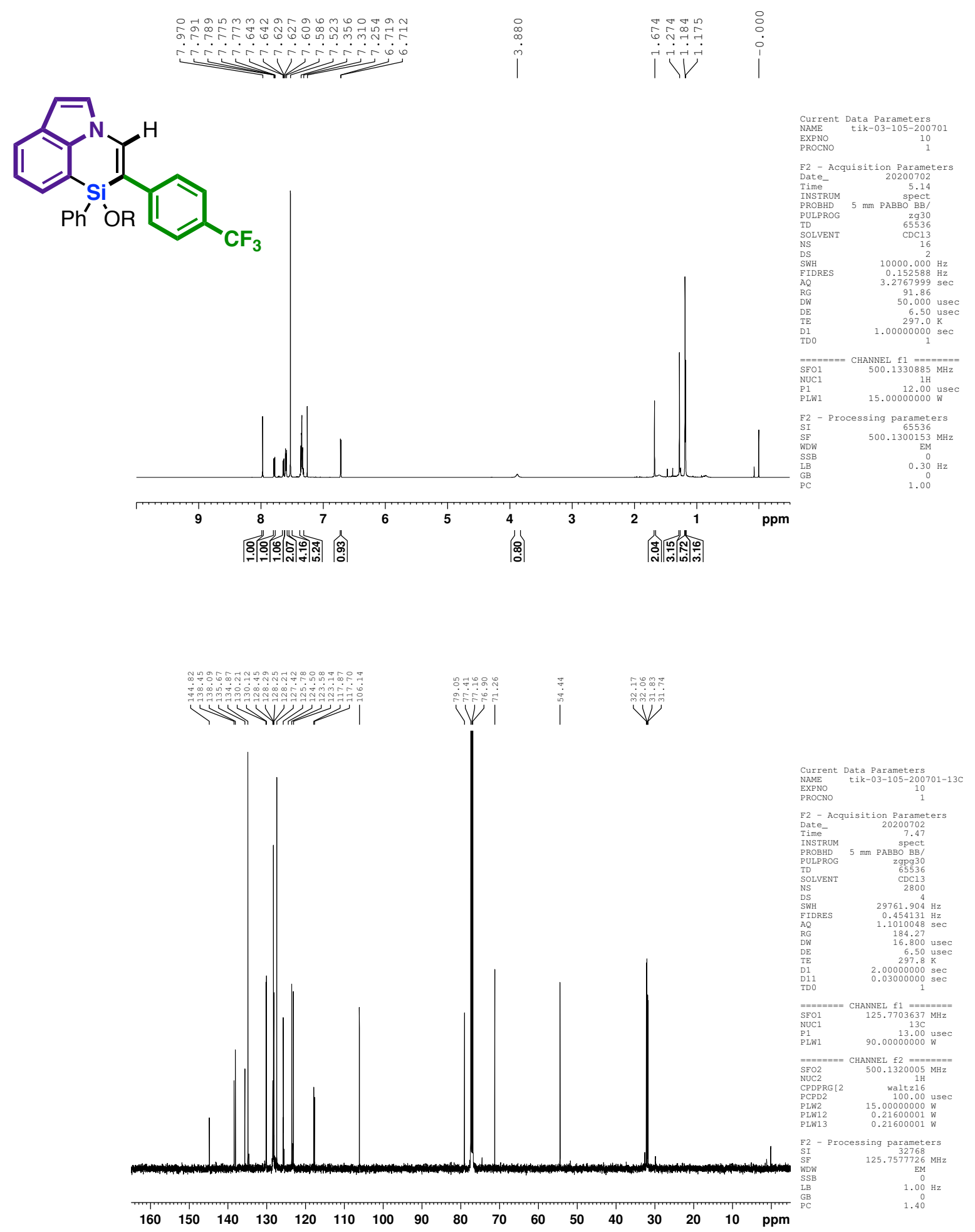


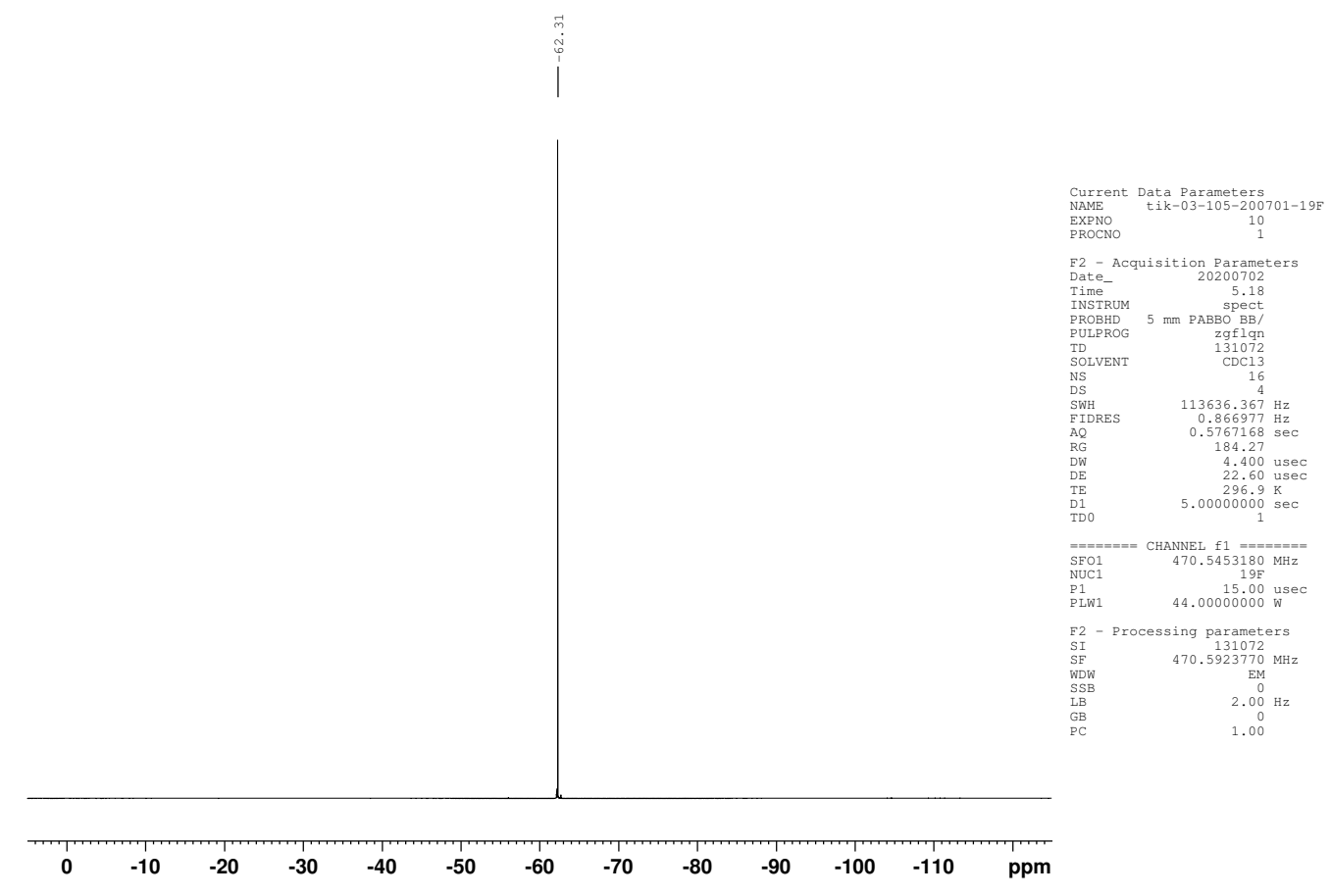

S-105 